\title{
Upregulation of Alpha Globin Promotes Apoptotic Cell Death in the Hematopoietic Cell Line FL5.12
}

Inauguraldissertation

zur Erlangung der Würde eines Doktors der Philosophie vorgelegt der Philosophisch-Naturwissenschaftlichen Fakultät der Universität Basel

von

Karin Brecht aus Bocholt (Deutschland)

Basel, 2004 
Genehmigt von der Philosophisch-Naturwissenschaftlichen Fakultät auf Antrag von

Prof. Dr. phil. Jutta Heim, Prof. Dr. phil. Peter Erb, Prof. Dr. phil. Thomas A. Bickle

Basel, den 21.09.2004

Prof. Dr. phil. Marcel Tanner 
Meinen Eltern und meiner Schwester gewidmet 



\section{Dank}

Ich danke Prof. Dr. Jutta Heim, die mir die Möglichkeit gegeben hat, meine Doktorarbeit in ihrer Gruppe in der Novartis Pharma Forschung durchzuführen. Ich bin im besonderen dankbar für ihre Anleitung und ihre Ratschläge.

Bedanken möchte ich mich bei Prof. Peter Erb für sein grosses Interesse und seine freundliche und akademische Unterstützung.

Sehr dankbar bin ich Dr. Marjo Simonen, die mit ihrem Enthusiasmus, den zahlreichen, spannenden Diskussionen und dem Korrekturlesen ganz besonders zum Gelingen der Arbeit beigetragen hat.

Ich möchte Brigitte Besenreuther danken für die Beta Globin Klonierung, aber besonders für ihre Fröhlichkeit, ihre aufmunternden Gespräche, und die schönen, gemeinsamen Laborstunden.

Ich danke Marion Kamke für die Erstellung der Affymetrix Chips, die mir viele wichtige Hinweise gegeben haben, und Alain Schilb für seinen Rat in Proteinfragen. Weiterhin danke ich Maja Walker (Novartis) und Prof. Radek Skoda (Universität Basel) für die Einschätzung des Differenzierungsgrades der FL5.12 Zellen.

Danken möchte ich Tony O'Sullivan, der sich die Zeit genommen hat, meine Arbeit sprachlich und grammatikalisch zu korrigieren.

Ich danke meinen Freunden in Basel und Bocholt, die mir immer wieder Mut zugesprochen haben und mit denen ich eine wunderbare Zeit in freien Stunden verbracht habe.

Ganz besonders danke ich Adrian, der mich in den vergangenen Jahren so liebevoll unterstützt hat. Seine Anregungen und Ratschläge, aber vor allem die gemeinsame Zeit mit ihm sind mir sehr wertvoll. Danken möchte ich auch Lisbeth und Max, die mich in den letzten Monaten meiner Arbeit mit ihrer Freundlichkeit und Wärme sehr aufgemuntert haben.

Mein grosser Dank geht an Mama, Papa und Ulrike! Ihrer Liebe und Unterstützung sind die Grundlage für meine Ideen und meine Arbeit. 


\section{Table of contents}

Summary

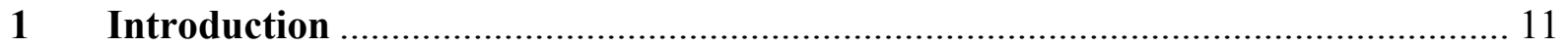

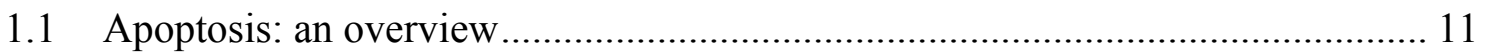

1.1.1 Apoptosis, necrosis, autophagy …...................................................... 11

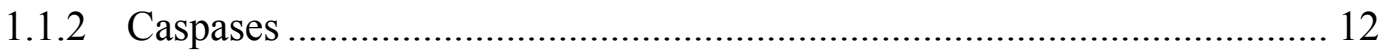

1.1.3 Intrinsic and extrinsic apoptotic signaling ......................................... 13

1.1.4 Bcl-2 family: regulators of apoptosis ............................................... 17

1.1.5 Survival and apoptotic signaling in hematopoietic FL5.12 cells ............. 19

1.2 Globin protein family and hematopoietic transcription factors ........................... 21

1.2.1 The globin protein family - Expression and function of alpha globin

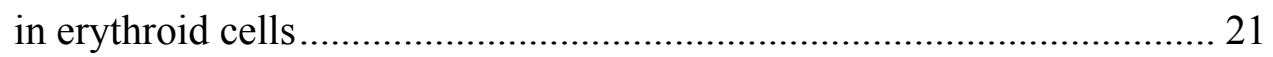

1.2.2 The globin gene locus, transcriptional regulation, and mRNA

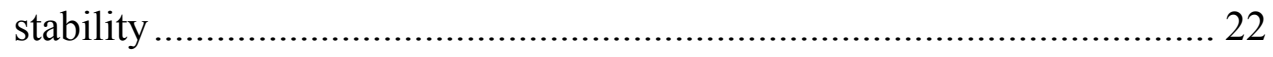

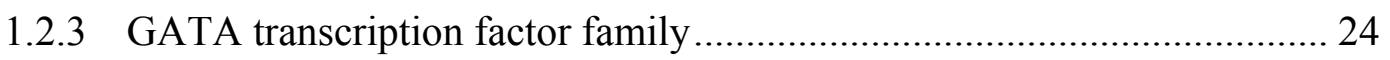

1.2.4 Assembly of hemoglobin and globin diseases ..................................... 26

1.2.5 Heme and globin detoxifying proteins, HO-1 and AHSP ....................... 26

1.2.6 Recent findings on novel functions of alpha globin.............................. 27

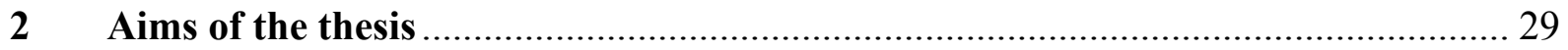

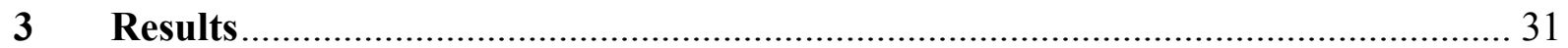

3.1 Upregulation of alpha globin promotes apoptotic cell death in the hematopoietic cell line FL5.12.

3.1.1 Alpha globin mRNA is highly expressed and upregulated in cytokine-deprived FL5.12 cells ......................................................... 31

3.1.2 Other globins or globin-like genes are expressed at low or undetectable levels

3.1.3 Alpha globin protein is upregulated upon IL-3 deprivation..................... 33

3.1.4 Alpha globin is localized to the cytoplasm and partly associates with the cytoskeleton after IL-3 deprivation

3.1.5 Differential expression of alpha globin after cisplatin, doxorubicin, staurosporine, and cycloheximide/TNF alpha treatment........................ 37

3.1.6 Alpha globin is not associated with heme ............................................ 39

3.1.7 Cytokine-deprived FL5.12 cells neither produce hemoglobin nor differentiate down the erythroid lineage ............................................... 41

3.1.8 AHSP does not change the viability of FL5.12 cells ............................ 43

3.1.9 NIH3T3, HeLa, and K562 cells differentially express alpha globin upon induction of apoptosis ............................................................... 45

3.1.10 GFP-alpha globin expressing cells are difficult to enrich ...................... 47 
3.1.11 GFP-alpha globin accumulates in the cytoplasm and tends to aggregate in FL5.12 cells

3.1.12 GFP-alpha globin is not associated with heme.

3.1.13 GFP-alpha globin accelerates apoptosis induced by IL-3 deprivation in a dose-dependent manner

3.1.14 GFP-alpha globin accelerates cell death in cisplatin-treated NIH3T3 cells.....

3.1.15 GFP-alpha globin reduces upregulation of endogenous alpha globin under cytokine-deprived conditions.

3.1.16 Pan caspase inhibitor, zVAD-fmk, delays apoptosis in FL5.12 cells expressing GFP-alpha globin and GFP-Bax

3.1.17 Caspase-3 and -9 are highly activated, caspase- 8 is moderately activated in GFP-alpha globin overexpressing cells in the absence of IL-3

3.1.18 Cytochrome c, Bid, and Bax are processed with faster kinetics in GFP-alpha globin expressing cells

3.1.19 Antisense alpha globin decreases basal levels of alpha globin in FL5.12 cells and reduces induction of alpha globin under cytokinedeprived conditions.

3.1.20 Antisense alpha globin delays caspase-3 activation but not DNA fragmentation in FL5.12 cells

3.2 Hematopoietic transcription factor GATA-2 promotes upregulation of alpha globin and cell death in FL5.12 cells.

3.2.1 GATA-2 transcription factor is significantly upregulated in cytokine-deprived FL5.12 cells

3.2.2 GATA-1 and -2 are differentially expressed in various cell lines upon induction of apoptosis.

3.2.3 NIH3T3, but not FL5.12 cells, ectopically expressing GATA-2, can be enriched....

3.2.4 Ectopically expressed GATA-2 is localized to the nucleus in FL5.12 and NIH3T3 cells

3.2.5 Overexpression of GATA-2 induces cell death in FL5.12 cells and causes a transient stress phenotype in NIH3T3 cells

3.2.6 Ectopically expressed GATA-2 induces alpha globin expression in FL5.12 but not NIH3T3 cells.

3.2.7 Antisense GATA-2 prevents upregulation of GATA-2 and reduces upregulation of alpha globin under apoptotic conditions in FL5.12 cells .....

3.2.8 Antisense GATA-2 delays DNA fragmentation in FL5.12 cells deprived of IL-3 
3.2.9 Overexpression or silencing of GATA-2 does not affect GATA-1

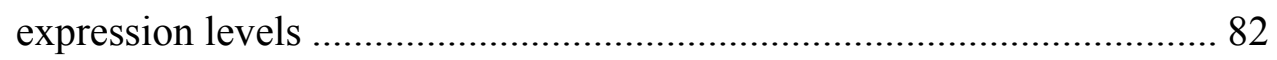

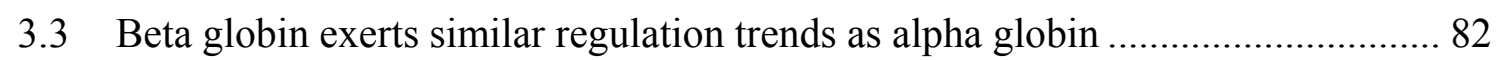

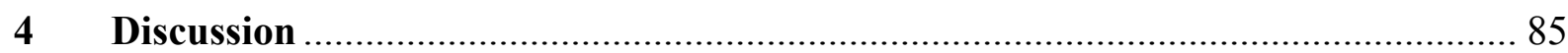

4.1 Novel function of alpha globin promotes apoptotic cell death ............................. 85

4.1.1 Characterization of endogenous alpha globin in PCD .......................... 85

4.1.2 Effects of overexpression and silencing of alpha globin in PCD............. 88

4.2 GATA-2 promotes upregulation of alpha globin and apoptosis in FL5.12

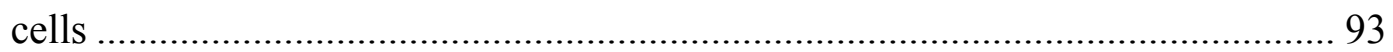

4.2.1 Expression and regulation of hematopoietic transcription factors in

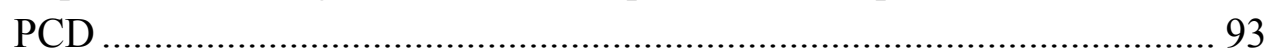

4.2.2 Effects of overexpression and silencing of GATA-2 on apoptosis and alpha globin expression ................................................................. 94

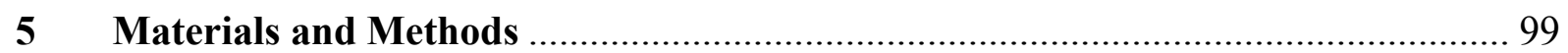

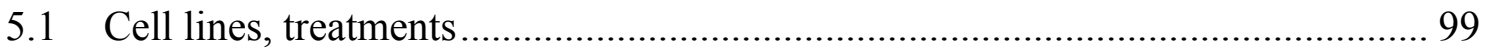

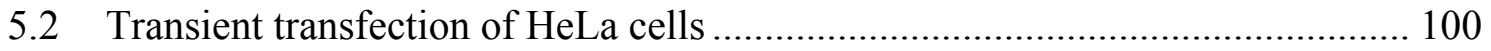

5.3 Gateway expression cloning and retroviral transduction ................................. 100

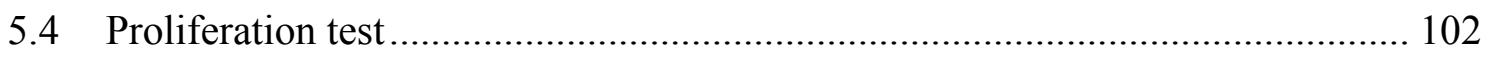

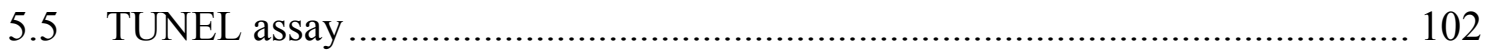

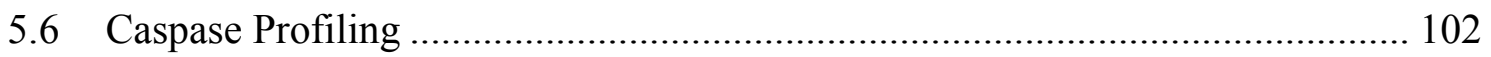

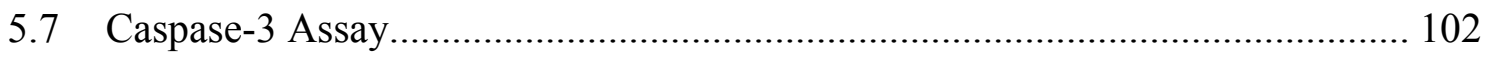

5.8 CellTiter-Glo ${ }^{\mathrm{TM}}$ Luminescent Cell Viability Assay .......................................... 102

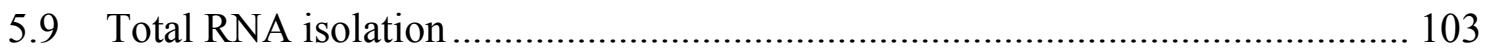

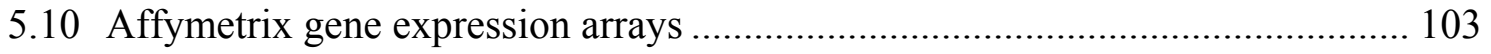

5.11 Quantitative polymerase chain reaction, RT QPCR …................................... 103

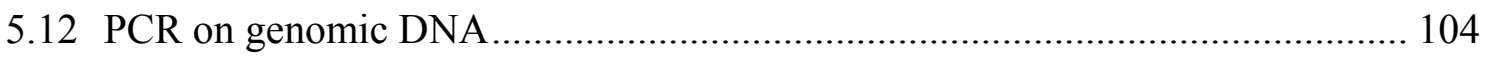

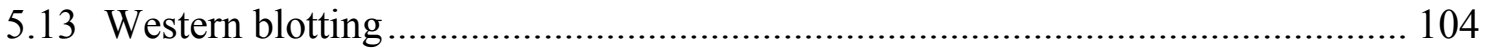

5.14 Immunostaining, DAPI staining and fluorescence microscopy ....................... 106

5.15 Determination of heme, hemoglobin, and differentiation stage of FL5.12

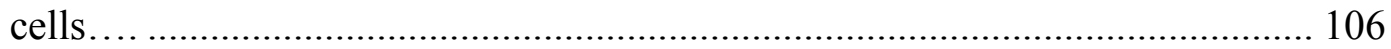

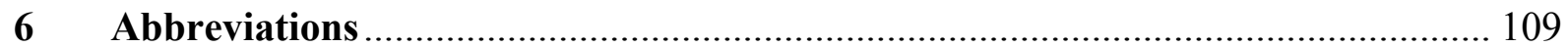

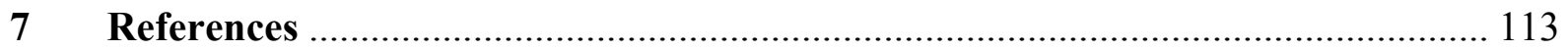




\section{Summary}

The function of alpha globin in the context of oxygen transport in erythroid cells is well described. There is growing evidence, however, that alpha globin is involved in processes other than tissue oxygen supply. Recently the expression of alpha globin has been shown to be induced upon specific apoptotic stimuli like cytokine deprivation or cisplatin treatment in the IL-3 dependent hematopoietic pro B cell line, FL5.12. In the current work we confirm for the first time that the upregulation of alpha globin is not restricted to the transcript, but is also observed at protein level. Different from erythroid cells, which bear globin proteins at $14 \mathrm{kD}$, alpha globin displayed two distinct bands of 14 and $14.5 \mathrm{kD}$ molecular weight under normal growth conditions. In healthy cells both forms of alpha globin were localized to the cytoplasm, whereas in apoptotic cells the $14 \mathrm{kD}$ alpha globin was partially localized to the cytoskeleton. In contrast to alpha globin, beta globin was expressed at a very low level, while other globins or globin-like genes were not expressed at all. Further, we found that alpha globin was not associated with its prosthetic group heme. Apoptotic cells neither produced hemoglobin nor displayed a phenotype of cells differentiating down the erythroid lineage. Very interestingly, also other cell lines of variable differentiation status including NIH3T3, HeLa, and K562 upregulated alpha globin during treatment with apoptosis-inducing agents indicating that alpha globin upregulation in apoptosis is not unique to FL5.12 cells. Enrichment of FL5.12 cells ectopically expressing GFP-alpha globin turned out to be difficult even in the presence of IL-3. Under IL-3-deprived apoptotic conditions, GFP-alpha globin accelerated the progression of cell death in a comparable fashion to GFP-Bax. In particular, caspase- $8,-9$ and -3 as well as the proapoptotic factors Bid, Bax, and cytochrome c were activated. In contrast, the expression of an antisense alpha globin construct exhibited a minor effect on the progression of apoptotic cell death. Taken together these data indicate that alpha globin is a new and crucial factor in apoptosis especially supporting the mitochondrial pathway, and its upregulation is a widespread phenomenon in apoptosis.

To study how the transcription of alpha globin is placed in the broader context of apoptosis, we searched for transcription factors, which were concomitantly upregulated with alpha globin. In cytokine-deprived FL5.12 cells transcription factor GATA-2, containing binding sites for regulatory sequences of globin and virtually all erythroid genes, was the most prominently upregulated candidate as assessed by gene chip arrays and RT QPCR. GATA-1 was expressed at low levels and weakly induced, while GATA-3 was completely absent. In FL5.12 cells treated with cisplatin or doxorubicin, GATA-2 levels remained unchanged. By investigating other cell lines, which induced alpha globin in apoptosis, we found that GATA2 was also upregulated in NIH3T3 but not K562 and HeLa cells. To evaluate the influence of GATA-2 on alpha globin expression and cell viability we overexpressed GATA-2 in FL5.12 and NIH3T3 cells. Interestingly, high expression of GATA-2 resulted in immediate cell death in FL5.12 cells and caused a severe but transient stress phenotype in NIH3T3 cells. We further found that alpha globin levels were indeed elevated in GATA-overexpressing FL5.12 but not NIH3T3 cells. Transduction of antisense GATA-2 in FL5.12 cells reduced both the increase of GATA-2 and alpha globin under apoptotic conditions and delayed cell death. In summary, our results suggest that the mechanisms to induce alpha globin under apoptotic 
conditions differ depending on the death stimulus and the cell line. The fact that overexpressed GATA-2 promoted, while antisense GATA-2 delayed cell death in FL5.12 cells, suggests that the function of GATA-2 is not restricted to maintenance and proliferation of immature hematopoietic progenitors but is also critical in apoptosis.

Publications:

Brecht, K.; Simonen, M.; Brachat, A.; Heim, J.; „Upregulation of alpha globin promotes apoptotic cell death in the hematopoietic cell line FL5.12“, Cell Death and Differentiation, 2004, submitted for publication.

Brecht, K.; Simonen, M.; Kamke, M.; Heim, J.; „Hematopoietic transcription factor GATA-2 promotes expression of alpha globin and cell death in FL5.12 cells“, Cell Death and Differentiation, 2004, submitted for publication.

Brachat, A.; Pierrat, B.; Xynos, A.; Brecht, K.; Simonen, M.; Brungger, A.; Heim, J. “A microarray-based, integrated approach to identify novel regulators of cancer drug response and apoptosis", Oncogene, 2002. 


\section{$1 \quad$ Introduction}

\subsection{Apoptosis: an overview}

\subsubsection{Apoptosis, necrosis, autophagy}

Apoptosis or programmed cell death (PCD) is a physiological process that has been described in all multicellular organisms studied so far, including plants, slime molds, nematodes, insects, and vertebrates (Ellis and Horvitz, 1986; Vaux et al., 1994). There is growing evidence that some form of PCD also exists in unicellular organisms like trypanosomes and bacteria (Yarmolinsky, 1995). PCD is a fine-tuned process, controlled by many extra- and intracellular signals, that coordinates the removal of hazardous or damaged cells (Savill et al., 1993) in order to maintain tissue homeostasis (Thompson, 1995). Disturbance of the apoptotic homeostasis is known to cause severe pathological conditions. Diseases linked with suppression of apoptosis include cancer (Zornig et al., 2001) or viral infections (Gregory et al., 1991) whereas increased apoptosis is observed in ischemic injury or neurodegenerative disorders (Pravdenkova et al., 1996).

In the early seventies Kerr and coworkers provided evidence that at least two distinct types of cell death exist. On the one hand necrosis was described as an accidental, uncontrolled type of degeneration that affects large cell populations. Morphologically, necrosis is characterized by cytoplasm swelling, destruction of organelles, disruption of the plasma membrane, and nonspecific degradation of DNA leading to the release of intracellular contents and inflammation (Kerr et al., 1972). On the other hand apoptotic cells are characterized by cell shrinkage, maintenance of organelle integrity, exposure of phosphatidylserine on the plasma membrane, membrane blebbing, lamin degradation (Rao et al., 1996), chromatin condensation and internucleosomal cleavage of DNA (Wyllie, 1980) followed by ordered removal through phagocytosis (Fadok et al., 1992). However, there are exceptions to this simple model, and there is growing evidence for alternative cell death pathways such as autophagy (Seglen and Bohley, 1992), as well as for intersections between intracellular mechanisms involved in distinct forms of cell death. Autophagy is a process that controls the turnover of organelles in many eukaryotic cell types and also in protozoas (Bera et al., 2003). Organelles and other cellular components are sequestered and degraded in lysosomes (Dunn, Jr., 1990) in order to resist starvation or as part of cell remodeling during differentiation, aging, and cell death (Klionsky and Emr, 2000). For example, MCF-7 breast carcinoma cells lacking caspase-3 were found to undergo autophagy rather than apoptosis after tamoxifen treatment (Bursch et al., 1996). Neurons of the substantia nigra in Parkinson patients displayed features of both autophagy and apoptosis (Anglade et al., 1997). Similarly, apoptotic and necrotic pathways have been reported to overlap to some extent. As an example, antiapoptotic Bcl-2 and caspase inhibitors were both able to delay necrotic cell death induced by cyanide or antimycin A (Shimizu et al., 1996). Further, internucleosomal fragmentation of DNA has also been observed during necrosis (Dong et al., 1997). In summary, the existence of alternative forms of cell death and possible intersections between these pathways hamper an easy classification. 


\subsubsection{Caspases}

One of the first organisms in which apoptosis was studied is the nematode Caenorhabditis elegans (C. elegans). Cell death defective (ced) genes play a critical role in the execution steps of apoptosis. Ced-3 and -4 promote PCD (Ellis and Horvitz, 1986), whereas Ced-9 inhibits the onset of apoptosis (Hengartner et al., 1992). The mammalian homologues are interleukin converting enzyme (ICE), apoptotic protease activating factor (Apaf-1), and Bcl-2, respectively. The Ced-3 protein belongs to a class of cysteine proteases that specifically cleaves after aspartate residues, which is why they are called caspases (cysteine aspartyl protease). According to phylogenetic analyses, all caspases can be divided into three subfamilies which differ significantly in substrate specificity and caspase function: (1) the ICE subfamily of cytokine processors including caspase-1 and its homologues caspase-12, 13 , and -14 , and further caspase-4, -5 , and $-11,(2)$ the ICH-1/Nedd-2 subfamily of apoptotic initiators comprising caspase-2, $-8,-9$, and 10 and the (3) CPP32 subfamily of apoptotic executioners, caspase-3, -6, and -7 also referred to as "downstream" or "effector" caspases (Alnemri et al., 1996; Van de et al., 1997). In general, caspases are synthesized as inactive proenzymes (zymogens) composed of three domains: an N-terminal prodomain, and the p20 and p10 domains. The prodomains of initiator caspases are long compared to the prodomains found in caspase-3, -6 , and -7 and play an important role in caspase regulation and function as signal integrators for apoptotic signals (Harvey et al., 1997). Caspase- 8 and -10 for instance contain a death effector domain (DED) that mediates the interaction via an adaptor molecule with the cytoplasmic tail of members of the tumor necrosis factor receptor (TNF-R1) family. Upon binding of Fas ligand or TNF alpha to their respective receptors, Fas (CD95) and TNF$\mathrm{R} 1$, apoptosis is triggered by the recruitment of a cytosolic adaptor molecule, FADD (MORT1), to the plasma membrane. FADD contains a DED that binds to the N-terminal DED prodomain of caspase-8 also called FLICE. (Muzio et al., 1996). Caspases-1, -2, -4, and -9 possess a prodomain called caspase recruitment domain (CARD) which most probably derives from the same ancestral domain as DED and mediates the interaction and activation of caspases with upstream regulators (Hofmann et al., 1997).

During caspase activation the prodomain is removed and the zymogen is further proteolytically cleaved between the p20 domain containing the catalytic cysteine residues and the p10 domain which determines substrate specificity (Walker et al., 1994). The mature caspase is a heterotetramer consisting of two p20/p10 heterodimers (Earnshaw et al., 1999). While initiator caspases are activated via oligomerization-induced autoprocessing (Butt et al., 1998), executioner caspases are activated by initiator caspases (Green, 1998) or by noncaspase proteinases such as granzyme B, a serine protease, which is introduced into cells by cytotoxic T-lymphocytes and natural killer cells (Darmon et al., 1995).

In some cases PCD has been reported to occur independently of caspase activation. Apoptosis inducing factor (AIF) for instance triggers cell death slightly differently from the standard apoptotic process and appears to act independently of caspase activation (Susin et al., 1999b). Alternatively, cell death involving the activation proteases other than caspases, such as calpain or cathepsins, has been observed (Squier et al., 1994; Deiss et al., 1996). Very 
recently cathepsins have been found to cleave pro-apoptotic Bid and to mediate cytochrome $\mathrm{c}$ release (Cirman et al., 2004).

There is growing evidence that the role of caspases is not exclusively restricted to apoptosis. Caspase-14 for instance exerts caspase-specific functions but is involved in terminal differentiation of keratinocytes and in normal skin formation (Van de et al., 1998; Lippens et al., 2003). Weakly activated caspase-9 partially cleaves RasGAP and generates an N-terminal fragment that exhibits potent anti-apoptotic signals. Strong caspase-9 activation results in RasGAP cleavage products that efficiently promote cell death (Yang and Widmann, 2001).

\subsubsection{Intrinsic and extrinsic apoptotic signaling}

Depending on the origin of the death signal and the initiator caspases involved, two major pathways can be distinguished: (1) the intrinsic or mitochondrial pathway that requires de novo transcription and translation and (2) the extrinsic or death receptor signaling pathway which is independent of macromolecule synthesis. The intrinsic apoptotic process occurs as a consequence of cellular stress and is mediated by the release of cytochrome c from the mitochondria. It additionally requires the induction of specific genetic programs by transcription factors. The extrinsic apoptosis is signaled via death receptors and occurs via recruitment and activation of caspases. Caspases, which exist as unprocessed zymogens and become cleaved and activated upon induction of apoptosis, play a central role in this pathway (Cerretti et al., 1992). In the extrinsic pathway, the mitochondrion-dependent signaling can be employed as an amplification loop (Kuwana et al., 1998).

Induction of apoptosis triggered by stress of the endoplasmatic reticulum and involving caspase-12 has been proposed as a third death pathway (Nakagawa et al., 2000).

Various signals can trigger the mitochondrial pathway via release of harmful proteins such as AIF, Smac/DIABLO (direct inhibitors of apoptosis (IAP) binding protein), Endonuclease G, procaspases or cytochrome $\mathrm{c}$ from the mitochondrial intermembrane space. Released AIF translocates to the nucleus and induces chromatin condensation in a caspase-independent manner (Susin et al., 1999b). Smac/DIABLO binds and inhibits IAPs which in turn lose their ability to bind and inactivate both procaspases and active caspases (Verhagen et al., 2000). Released procaspase-2, -3 and -9 , are processed to generate enzymatically active caspases (Susin et al., 1999a). Under normal growth conditions cytochrome c acts as a specific and efficient electron transfer mediator of the mitochondrial respiratory chain. However, when apoptosis is induced cytochrome c becomes part of the apoptosome, a complex consisting of Apaf-1, dATP or ATP, and procaspase-9 (Li et al., 1997). While Apaf-1 was initially thought to be only transiently required for caspase- 9 activation, it has been suggested that the Apaf$1 /$ caspase- 9 complex is the active form of caspase-9 (Rodriguez and Lazebnik, 1999). In this model the caspase-9 holoenzyme activates the downstream caspase effector cascade involving caspase-3, -6, and -7 . In cell-free systems caspase-2, -8, and -10 were also found to be activated by caspase-9 (Slee et al., 1999). Recent co-immunoprecipitation results demonstrate that regulatory proteins such as Smac/DIABLO associate with the apoptosome and regulate the activity of native apoptosomes, whereas cytochrome $\mathrm{c}$ is not stably associated with the 
complex (Hill et al., 2004). Also very recently caspase-2 has been found to directly trigger mitochondria-mediated cytochrome $\mathrm{c}$ release and this independently of its enzymatic activity (Robertson et al., 2004).

One feature of the mitochondrial pathway that is now understood is the caspase-dependent drop of mitochondrial inner transmembrane potential, $\Delta \psi \mathrm{m}$, following the cytochrome c release (Zamzami et al., 1995; Goldstein et al., 2000). The collapse of $\Delta \psi \mathrm{m}$ indicates the opening of a large conductance channel, the mitochondrial permeability transition pore (PTP), which includes both inner membrane proteins such as the adenine nucleotide translocator (ANT), and outer membrane proteins, such as porin also known as voltage-dependent anion channel (VDAC).

Several competing models exist to explain mitochondrial membrane permeabilization and subsequent release of pro-apoptotic molecules during apoptosis (Martinou et al., 2000). Two models claim the outer mitochondrial membrane rupture to be the result of the mitochondrial matrix swelling. The first model involves opening of the permeability transition pore (PTP) protein complex spanning the inner and outer mitochondrial membranes by PTP openers like Bax (Marzo et al., 1998). The other model postulates a defect in ATP/ADP exchange as a result of closing of VDAC, causing hyperpolarisation of the inner mitochondrial membrane and mitochondrial swelling (Green and Kroemer, 1998).

As an alternative to the mitochondrial membrane damage models, the formation of membrane pores has been proposed. Again different hypotheses were made to explain pore formation and subsequent release of cytochrome $\mathrm{c}$ and other apoptotic molecules. The first model assumes that oligomers of Bax form a channel in the mitochondrial membrane (Schlesinger et al., 1997). According to Basanez and colleagues, Bax destabilizes the phospholipid bilayer structure and forms lipidic pores (Basanez et al., 1999). The last model suggests that Bax binds VDAC and changes its conformation leading to the formation of a cytochrome cpermeable channel (Shimizu et al., 1999).

All models share the common prediction that cytochrome $\mathrm{c}$ release is controlled by proteins of the Bcl-2 family which are also found in the outer mitochondrial membrane. Those that inhibit cell death, Bcl-2 and $\mathrm{Bcl}-\mathrm{x}_{\mathrm{L}}$, prevent the release of cytochrome $\mathrm{c}$ whereas those promoting cell death like Bax and Bak, induce its release (Pan et al., 1998) (see below).

Death receptor signaling represents the second major apoptotic pathway. Death receptors belong to the tumor necrosis factor receptor (TNF) superfamily consisting of approximately 30 proteins, which are involved in a broad range of biological functions including regulation of apoptosis and survival, differentiation and immune response (reviewed in (Ashkenazi and Dixit, 1998; Walczak and Krammer, 2000)). In general, mammalian TNF-R family members are primarily type I transmembrane proteins and share similar extracellular cysteine-rich domains. Several death receptors like TNF-R1, Fas/APO-1/CD95, DR3, DR6, TRAILR1/DR4 and TRAIL-R2/DR5 possess a common cytosolic domain of about 80 amino acid referred to as death domain (DD) (Itoh and Nagata, 1993). The death domain is essential for transmitting the death signal from the cell surface to intracellular signaling pathways. In 
contrast, death receptors such as TNF-R2, CD40, LT- $\beta$ R, CD27 or CD30 lack this cytosolic death domain and are believed to synergistically enhance TNF-R1-induced cytotoxicity (Tartaglia et al., 1993; Grell et al., 1999). In addition to typical membrane-spanning death receptors, soluble forms are generated by both alternative splicing (Cascino et al., 1995) and by proteolytic processing (Schall et al., 1990). Functional TNF-Rs are typically oligomeric, most probably trimeric complexes stabilized by disulfide bonds (Song et al., 1994). An Nterminal extracellular domain called pre-ligand binding assembly domain (PLAD) mediates receptor self-association before ligand binding (Chan et al., 2000) leading to the assumption that TNF-Rs exist as pre-formed complexes rather than individual receptor subunits that oligomerize after ligand binding.

Ligands of the TNF-R superfamily, except for lymphotoxin alpha, are synthesized as nascent type II membrane-associated proteins. Most ligands act as trimeric or multimeric membranebound proteins, however, also soluble forms can be generated by limited proteolysis by metalloproteinases (Tanaka et al., 1996). The ligands share a characteristic region of 150 amino acids towards the C-terminus called TNF homology domain. This region is responsible for receptor binding and subsequent activation. Further, agonistic antibodies can activate death receptor signaling and induce apoptosis (Ni et al., 1994).

The best-characterized death receptors comprise (1) CD95, (2) TNF-R1, and (3) TRAIL-R1/2. By referring to these three receptors major features of the death receptor pathways will be described in the following paragraphs:

(1) The biological role of the CD95/CD95L (Fas or APO-1) apoptotic pathway is best understood in the immune system where it is implicated in clonal depletion of T-lymphocytes, cytotoxic response or B-cell apoptosis induction. Upon binding of CD95L to its receptor, formation of a death-inducing signaling complex (DISC) is initiated. The CD95-DISC contains the cytoplasmic adapter, FADD, and procaspase-8 or -10 (Kischkel et al., 1995). CD95-mediated clustering within the DISC results in autoproteolytic processing of the procaspases by induced proximity and release of active caspases. Concerning DISC downstream signaling two types of cells can be distinguished: In type I cells processed caspase-8 alone is sufficient to activate other caspases (Scaffidi et al., 1998; Scaffidi et al., 1999). In contrast, CD95 signaling in type II cells depends on an amplification loop via mitochondria relying on caspase- 8 that mediates cleavage of pro-apoptotic Bid (Kuwana et al., 1998). Truncated Bid, tBid, translocates to mitochondria and promotes conformational change, oligomerization and recruitment of Bax to the mitochondria (Eskes et al., 2000). Subsequently, cytochrome $\mathrm{c}$ is released and the apoptosome is formed initiating a mitochondrial feedback loop (Luo et al., 1998). In addition, cleavage of caspase-6 downstream of mitochondria may initiate a positive feedback to the receptor pathway by cleaving caspase-8 (Cowling and Downward, 2002).

Interestingly, FADD and other DED containing proteins are not restricted to their function as adapter molecules in the cytosolic DISC complex. It has been recently reported that a nuclear localization signal in their DED recruits these proteins into the nucleus suggesting an 
independent, direct role in apoptosis (Stegh et al., 1998; Schickling et al., 2001; GomezAngelats and Cidlowski, 2003).

Naturally occuring inhibitors of the CD95 as well as TNF-R1 death signaling pathway are viral and cellular FLICE inhibitory proteins, the v- and c-FLIPs (Thome et al., 1997; Irmler et al., 1997). Human c-FLIP exists in two isoforms, FLIP $\mathrm{S}_{\mathrm{S}}$ and FLIP $_{\mathrm{L}}$, both of which are recruited to the CD95 DISC in a stimulation-dependent manner. FLIP $\mathrm{S}_{\mathrm{S}}$ inhibit apoptosis by blocking caspase- 8 activation. Another way cells inhibit death ligand induced apoptosis is via the soluble CD95 decoy receptor, DcR3. DcR3 has been shown to bind to CD95L and to inhibit CD95L-induced apoptosis (Pitti et al., 1998).

(2) TNF is a well characterized cytokine which exists as a homotrimer and is primarily produced by activated macrophages (Fransen et al., 1985). TNF signals through TNF-R1 and -2 and results in the activation of two pleiotropic transcription factors, nuclear factor kappa $\mathrm{B}$ (NF-kB) and c-Jun. The two transcription factors induce expression of genes that are implicated in diverse biological processes such as cell growth and death, development, oncogenesis, immune and stress response. Hence, inappropriate production of TNF is responsible for the pathogenesis of a wide range of human diseases, including rheumatoid arthritis, sepsis, cancer, and autoimmune response.

Upon engagement of TNF to its cognate receptor, the inhibitory protein, silencer of death domain (SODD), is released from the TNF-R1. This enables TNF to bind to the TNF-Rassociated death domain (TRADD). TRADD in turn recruits additional adapter proteins such as receptor-interacting protein (RIP), TNF-R-associated factor 2 (TRAF2), and FADD. These adaptors further recruit key enzymes that initiate the signaling events. While the protein kinase RIP degrades I $\mathrm{B}$ (inhibitor of NF- $\kappa \mathrm{B}$ ) thereby activating NF- $\kappa \mathrm{B}$ and suppressing apoptosis, FADD recruits caspase- 8 and initiates autocatalytic cleavage which activates the caspase cascade. TRAF2 (Rothe et al., 1994) in contrast attracts inhibitory apoptosis molecules like cellular inhibitor of apoptosis protein 1 and 2 (cIAP-1/-2), which act as endogenous inhibitors of caspases (Rothe et al., 1995). TRAF2 is further believed to activate a cascade of kinases that finally increases the transcriptional activity of c-Jun. In summary the outcome of TNF signaling can either be pro- or antiapoptotic, and depends on the availability of activating and inhibitory proteins and their relative affinities to the TNF-R signaling complex.

(3) TRAIL (TNF-related apoptosis-inducing ligand, APO-2L) is expressed in a wide range of tissues and transmits the death signal via TRAIL-R1/DR4 and TRAIL-R2/DR5 (Wiley et al., 1995). Similarly to CD95-mediated cell death TRAIL signaling results in receptor trimerization and clustering of death domains leading to the formation of a DISC and subsequent activation of caspase-8 (Bodmer et al., 2000) and -10 (Kischkel et al., 2001). TRAIL-R3 and TRAIL-R4 have been identified as decoy receptors that lack any functional death domain and are therefore unable to induce cell death (Pan et al., 1997). Instead they prevent TRAIL binding to DR4 and DR5. The existence of decoy receptors and the observation that TRAIL has different affinities to the TRAIL receptors may explain why some cells are preferentially killed by TRAIL whereas others are not (Truneh et al., 2000). 


\subsubsection{BCl-2 family: regulators of apoptosis}

Bcl-2 and related intracellular proteins are key regulators of apoptosis both in the intrinsic and extrinsic apoptosis signaling pathways. To date, more than $25 \mathrm{Bcl}-2$ family members have been identified (Adams and Cory, 1998; Borner, 2003). These can be divided into two classes: members that inhibit and members that promote cell death. Homeostasis is ensured by strictly controlling the amount of active pro- and antiapoptotic family members. Cellular stress disrupts this balance between pro- and anti-apoptotic proteins and leads to apoptosis.

Unrelated to their role in apoptosis, all Bcl-2 family members contain at least one of four conserved Bcl-2 homology (BH) domains, designated $\mathrm{BH} 1$ to $\mathrm{BH} 4$. These domains do not exhibit enzymatic activity but mediate the interaction of the Bcl-2 family members with other proteins. Many Bcl-2 proteins are further characterized by a hydrophobic C-terminal domain (TM) which is responsible for membrane anchorage and cellular localization (Nguyen et al., 1993). According to the BH and TM domains the following classification of the Bcl-2 family can be made: The anti-apoptotic members such as Bcl-2, Bcl- $\mathrm{x}_{\mathrm{L}}$ and Bcl-W show sequence conservation in all four $\mathrm{BH}$ domains and possess the TM domain. All pro-apoptotic proteins, in contrast, lack at least the BH4 domain. Further sequence homology studies lead to division into three groups: first, members containing the $\mathrm{BH} 1, \mathrm{BH} 2, \mathrm{BH} 3$ and the $\mathrm{TM}$ domain like Bax, Bak, and Bok; second, the "Bcl-alone" proteins possessing the BH3 and the TM domains (BNip, Blk); and third, the "BH3-only" proteins having only the BH3 domain (Bad, Bid, Bim) (Figure 1).

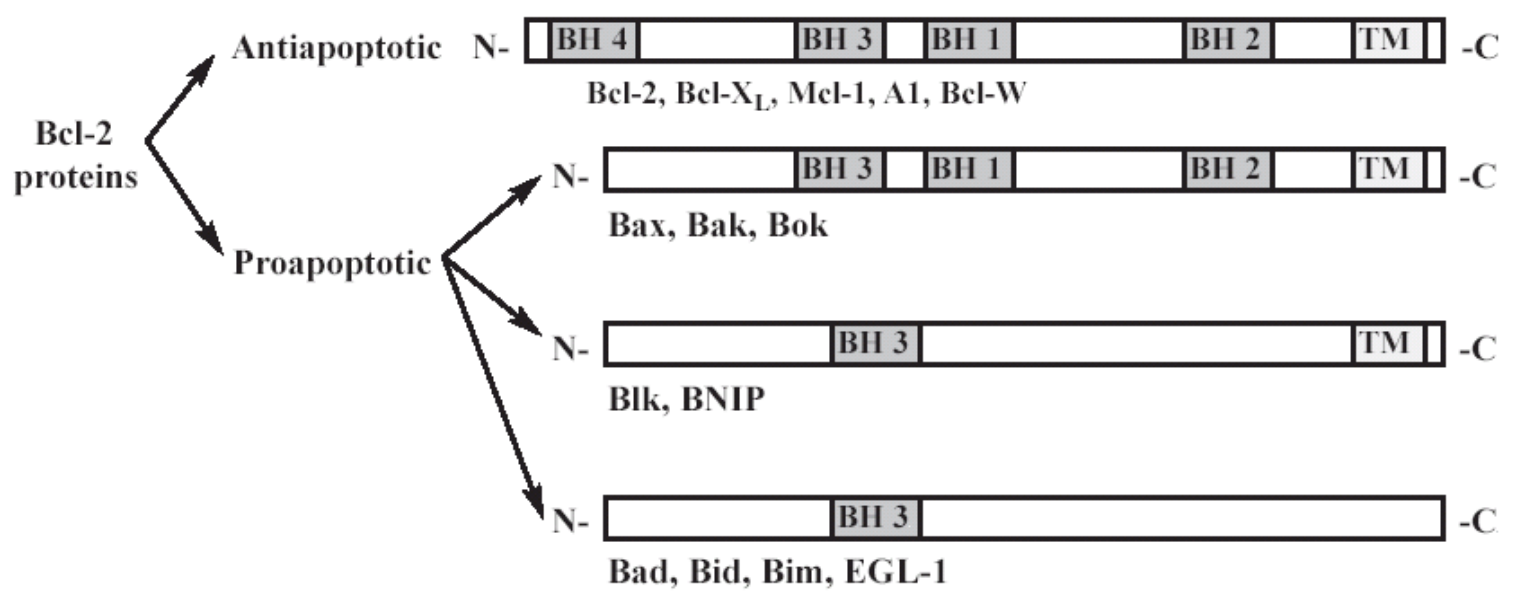

Figure 1: Schematic classification of Bcl-2 family proteins. Proteins are represented in linear forms. Bcl-2 homology domains are indicated by BH1 - BH4, the hydrophobic, C-terminal transmembrane domains by TM.

Anti- and pro-apoptotic proteins display different patterns of membrane localization. For instance, Bcl-2 is mainly membrane-bound and resides on the cytoplasmic face of mitochondrial outer membrane, the endoplasmic reticulum (ER), and the nuclear envelope 
(Krajewski et al., 1993). Especially mitochondrial membrane anchorage plays a key role in regulating mitochondrial membrane integrity and preventing apoptosis. $\mathrm{Bcl}-\mathrm{x}_{\mathrm{L}}$ is found on membranes as well as in the cytoplasm (Gonzalez-Garcia et al., 1994). In contrast, Bax is predominantly localized in the cytosolic fraction. Upon apoptosis induction, however, Bax exposes its membrane-anchoring domain and redistributes to the mitochondria and promote cytochrome c release (Hsu et al., 1997).

Three Bcl-2 family members, Bcl-2, Bcl- $\mathrm{x}_{\mathrm{L}}$, and Bax were more closely investigated in the context of the present thesis and will be briefly described in the next paragraph. The protooncogenic activity of Bcl-2 was first discovered in B-lymphoid cells. Rather than promoting cell proliferation or inhibiting differentiation like other oncogenes, it suppresses lymphocyte apoptosis (Vaux et al., 1988). Beside the ability of Bcl-2 to inhibit apoptosis by preventing cytochrome c release from mitochondria, two other mechanisms have been discussed. In analogy to findings in C. elegans, where CED-9, the nematode homologue of Bcl-2, has been found to interact directly with CED-4 and inhibit apoptosis, a direct role of Bcl-2 as well as $\mathrm{Bcl}-\mathrm{x}_{\mathrm{L}}$ in regulating the activation of caspases has been suggested (Chinnaiyan et al., 1997). Further, Bcl-2 plays an important role in calcium homeostasis in the endoplasmic reticulum. Maintenance of ER calcium by Bcl-2 has been found to promote cell proliferation and to inhibit apoptosis (He et al., 1997). Interestingly, Bcl-2 is targeted and cleaved by caspase-3 during apoptosis. The generated C-terminal cleavage product itself promotes apoptosis and is therefore part of a positive feedback loop within the death machinery (Kirsch et al., 1999).

$B c l-x$ encodes two polypeptides arising from alternative splicing. The longer mRNA encodes the death-suppressing Bcl- $\mathrm{x}_{\mathrm{L}}$ that has high functional similarity to $\mathrm{Bcl}-2$. It is transcriptionally

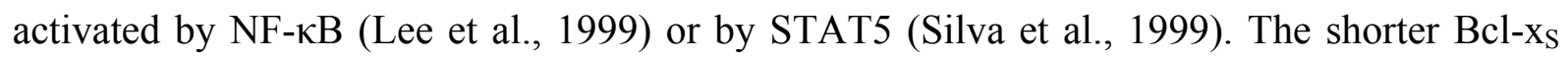
lacks the $\mathrm{BH} 1$ and $\mathrm{BH} 2$ and acts as a negative regulator of $\mathrm{Bcl}-\mathrm{x}_{\mathrm{L}}$ and $\mathrm{Bcl}-2$ (Boise et al., 1993).

Additional regulation of the apoptotic process is provided by the interaction of anti-apoptotic Bcl-2 and Bcl- $x_{\mathrm{L}}$ with the BH3 domain of the pro-apoptotic members of the Bcl-2 family (Chittenden et al., 1995). For instance, Bax and Bcl-2 form heterodimers and the ratio of Bcl-2 to Bax determines survival or death following an apoptotic stimulus (Oltvai et al., 1993). Bcl- $\mathrm{X}_{\mathrm{L}}$ is bound and antagonized by Bak (Sattler et al., 1997). However, mutation and deletion studies elucidated that both Bax and Bak are able to promote apoptosis by a heterodimerization-independent mechanism (Zha and Reed, 1997; Simonian et al., 1997). Bad heterodimerizes with $\mathrm{Bcl}-\mathrm{x}_{\mathrm{L}}$ and Bcl-2 and thereby displaces Bax from the anti-apoptotic proteins and promotes cell death (Yang et al., 1995).

Bax is the prototype of cell death-mediating Bcl-2 family members. The mechanism of Bax and Bak-mediated cell death was unknown for a long time. The current opinion is that they undergo conformational changes in response to a death signal, causing translocation, mitochondrial membrane insertion and homodimerization that finally results in cell death (Gross et al., 1998). One candidate responsible for the conformational changes of Bax (Desagher et al., 1999) and Bak (Wei et al., 2000) is the BH3 only protein, Bid. Bid is cleaved after induction of apoptotic cell death by CD95 death receptor signaling (see above). 
Interestingly, the human and mouse bax genes contain a p53 and p73 consensus binding site. Both transcription factors have been shown to mediate the expression of Bax after DNA damage (Miyashita and Reed, 1995). Further, the pro-apoptotic BH3 only proteins Noxa (Oda et al., 2000) and PUMA (p53-upregulated modulator of apoptosis) (Nakano and Vousden, 2001) have been described to be regulated by p53 and recently also by E2F1 transcription factor (Hershko and Ginsberg, 2004). Very recently PUMA has been identified as the transcriptional target of $\mathrm{p} 73$ and as an inducer of Bax translocation from the cytosol to the mitochondria (Melino et al., 2004).

\subsubsection{Survival and apoptotic signaling in hematopoietic FL5.12 cells}

In 1985 McKearn and colleagues generated the murine IL-3 dependent pro B cell line, FL5.12 (McKearn et al., 1985). B cells generate from pluripotent hematopoietic stem cells in the liver during mid-to-late fetal development and in the bone marrow after birth. FL5.12 cells are enriched mouse fetal liver cells that respond to interleukin 3 (IL-3), a multilineage hematopoietic growth factor. Fetal liver cells display high frequencies of multipotential erythroid and myeloid precursors (colony-forming unit mix, CFU-mix) or restricted erythroid and myeloid precursors such as granulocyte and macrophage CFU and erythroid burstforming unit as well as precursors that differentiate to become mature B lymphocytes. The Blymphocyte precursors can be cloned in single-cell cultures in the presence of IL-3. The doubling time is about $12 \mathrm{~h}$. Growth of these clones can be maintained for more than six months in the presence of IL-3. During culture IL-3 tightly controls IL-3R alpha and beta chain expression and the cells maintain their capacity to differentiate to mature $\mathrm{B}$ lymphocytes (Algate et al., 1994).

The PI3K/Akt signaling pathway is of central importance for maintenance of cell survival in FL5.12 cells (Minshall et al., 1996). Briefly, upon cytokine stimulation the p85 regulatory subunit of phosphatidylinositol 3-kinase (PI3K) associates with the IL-3 $R \beta_{C}$ chain and becomes phosphorylated. This results in activation of the p110 catalytic subunit of PI3K which phosphorylates membrane lipid phosphatidylinositol $(4,5)$-bisphosphate. The generated phosphatidylinositol $(3,4,5)$-tris-phosphates $\left(\mathrm{PI}(3,4,5) \mathrm{P}_{3}\right)$ serve to localize and activate phosphatidylinositol-dependent kinase (PDK-1) in the vicinity of the cell membrane. In turn Akt also called protein kinase $\mathrm{B}$ (PKB) is targeted to the lipid-rich cell membrane and becomes phosphorylated on T308 in the catalytic domain and S473 in the C-terminal domain (Wick et al., 2000). Akt prevents apoptosis by phosphorylating several downstream targets including Bad (Datta et al., 1997), forkhead transcription factor (Foxo-3) (Brunet et al., 1999), and caspase-9 (Cardone et al., 1998). Akt has also been found to inhibit the conformational changes of Bax and its redistribution to the mitochondrial membranes (Yamaguchi and Wang, 2001). While phosphorylated caspase-9 inhibits procaspase-9 processing in the apoptosome, phosphorylated Bad and Foxo-3 are sequestered by members of the 14-3-3 protein family in the cytosol. IL-3 deprivation results in dephosphorylation of Bad and Foxo-3 which in turn triggers the subsequent release of Bad and Foxo-3 from 14-3-3 and their activation. Tumor suppressor phosphatase and tensin homologue deleted on chromosome 10, shortly PTEN, reduces intracellular $\mathrm{PI}(3,4,5) \mathrm{P}_{3}$ levels by dephosphorylation and thereby negatively regulates the PI3K/Akt signaling pathway (Stambolic et al., 1998). 
Beside the PI3K/Akt signaling pathway, other pathways including the Ras/Raf/MEK/ERK and the Jak/STAT pathways are activated by IL-3. Central downstream phosphorylation targets of the first pathway are members of the p90 ribosomal S6 kinase family (p90S6K, Rsk) which phosphorylate and inhibit Bad as well as phosphorylating and transactivating the transcription factor cAMP response element-binding protein (CREB) (Bonni et al., 1999). CREB drives the expression of anti-apoptotic Bcl-2 family members (Wilson et al., 1996). The latter pathway signals phosphorylation and activation of cytoplasmic STAT transcription factors which translocate to the nucleus and induce genes essential for cytokine-regulated processes such as cellular proliferation, differentiation as well as survival (Mui et al., 1995). Intersections between different signaling pathways have been reported (Shelton et al., 2003). For instance, Raf has been shown to be recruited to the plasma membrane by Ras and to be then phosphorylated by Jak upon growth hormone stimulation. Raf has additionally been shown to be phosphorylated by Akt which results in inhibition of the Raf/MEK/ERK pathway (Zimmermann and Moelling, 1999). On the other hand it has been reported, that apoptosis suppression by Raf-1 and MEK1 requires both MEK- and PI3K-dependent signals (von Gise et al., 2001).

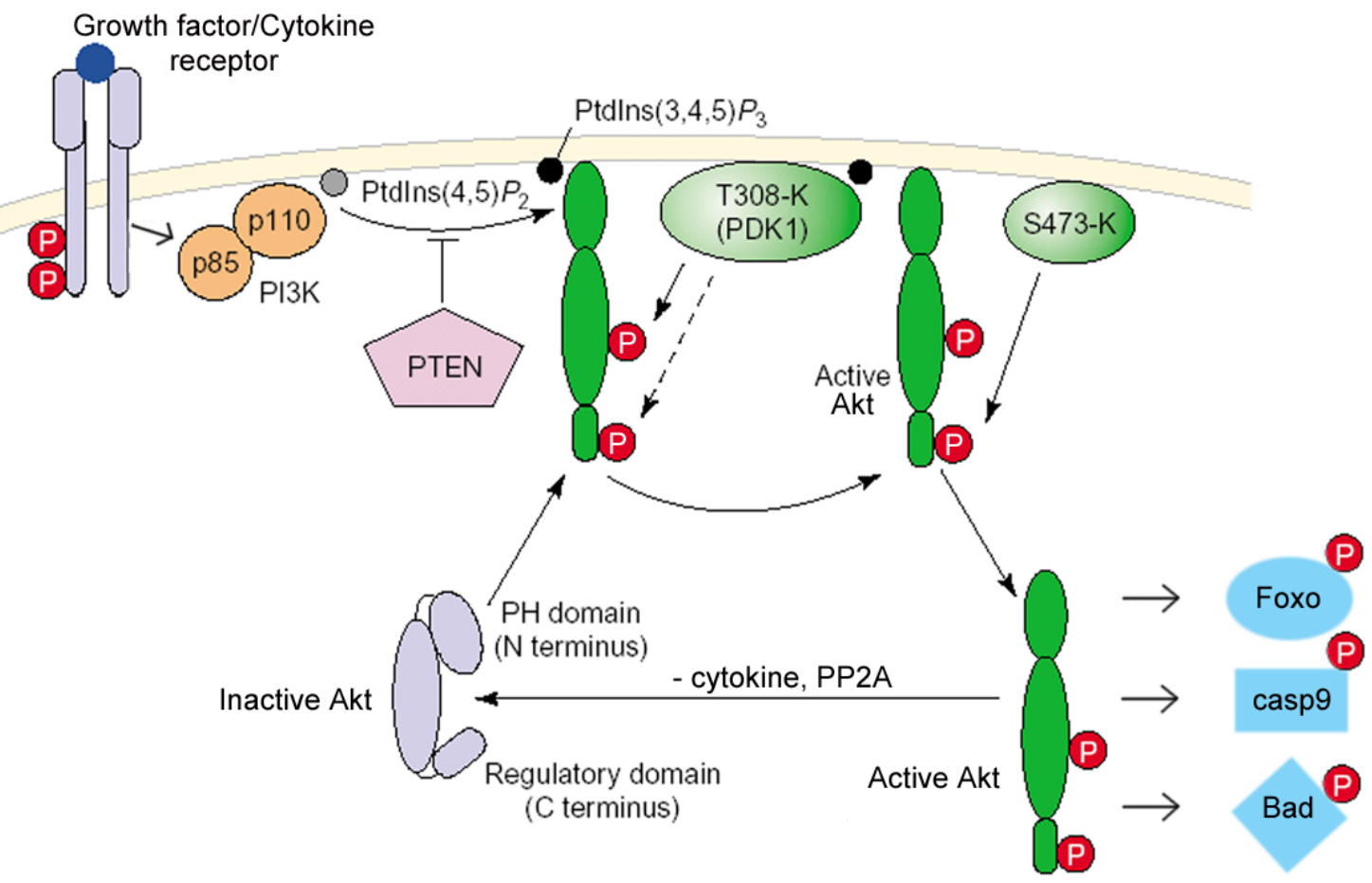

Figure 2: Proposed model for the PI3K/Akt survival signaling pathway. Activation of a receptor tyrosine kinase by a growth factor or of a cytokine receptor by the corresponding cytokine leads to recruitment and activation of PI3K. Activated PI3K generates 3 'phosphoinositides (grey and black circles) that in turn mediate the recruitment of Akt to the plasma membrane. Akt is then phosphorylated (phosphates are depicted with red circles) by PDK 1 and probably by other so far unidentified kinases. Activated Akt now mediates its intracellular survival effects, e.g. by phosphorylation and inhibition of Foxo, caspase-9 or Bad. PTEN limits the activation of Akt by dephosphorylating $\mathrm{PI}(3,4,5) \mathrm{P} 3$; protein phosphatase $2 \mathrm{~A}$ (PP2A) inhibits by direct dephosphorylation of Akt (redrawn and modified from (Brazil and Hemmings, 2001)). 


\subsection{Globin protein family and hematopoietic transcription factors}

\subsubsection{The globin protein family - Expression and function of alpha globin in erythroid cells}

Globin proteins have been found in many taxa including bacteria, plants, fungi, and vertebrates and belong to the large group of heme-containing proteins (Hardison, 1996). Heme is a protoporphyrin IX molecule with an iron ion bound in the middle of its flat, planar structure. Heme biosynthesis takes place in almost all animal cells except mature erythrocytes which lack a nucleus and all subcellular organelles. Heme synthesis requires four cytoplasmic and four mitochondrial enzymes including for example delta aminolevulinic acid synthetase (ALA-S), ferrochelatase or porphobilinogen deaminase (PBGD) (Sassa, 1976). 85\% of all heme is synthesized in erythroid cells where it reversibly binds oxygen. In erythroid cells the iron transporter transferrin is the only physiological iron source for heme synthesis (Ponka, 1997).

So far, the globin protein family comprises hemoglobins, myoglobin, neuroglobin, and the recently discovered cytoglobin (Burmester et al., 2002). Hemoglobin was the first complex protein whose three-dimensional structure was solved (Muirhead et al., 1967) and alpha globin ( $\alpha$-globin) the first gene to be cloned (Rabbitts, 1976). All hemoglobins are heterotetramers consisting of two alpha-globin-like and two beta-globin-like chains. Each globin chain binds one molecule of heme in a precise fashion in its hydrophobic pocket. Synthesis of hemoglobin occurs in the erythroblast and reticulocyte stages of erythrocyte development. Depending on the developmental stage the following forms of hemoglobin can be distinguished:

In the first 1-3 months of human fetal life two different types of embryonic hemoglobin are expressed in primitive erythroblasts, $\mathrm{Hb}$ Portland and $\mathrm{Hb}$ Gower1. Hb Portland consists of two alpha-like zeta chains $(\zeta)$, and two beta-like gamma chains $\left(\zeta_{2} \gamma_{2}\right)$, Hb Gower1, the predominant form of hemoglobin, consists of two zeta and two beta-like epsilon $(\varepsilon)$ chains $\left(\zeta_{2} \varepsilon_{2}\right)$ (Adachi et al., 2002). Later in definitive erythropoiesis the embryonic globins are replaced by fetal globin, $\mathrm{HbF}$ composed of two alpha and two gamma chains $\left(\alpha_{2} \gamma_{2}\right)$. Both the embryonic and the fetal forms of hemoglobin have a higher affinity for oxygen than the maternal hemoglobin, thus ensuring sufficient oxygen supply of the fetus (Dow et al., 1995). From one month before birth until the age of three months $\mathrm{HbF}$ is replaced by adult hemoglobin, $\mathrm{HbA}$ or $\mathrm{Hb}_{0}$, consisting of two alpha and two beta globin chains $\left(\alpha_{2} \beta_{2}\right)$.

Myoglobin ( $\mathrm{Mb}$ ) acts as a monomeric, 153 amino acid long protein and facilitates oxygen transport in the skeletal as well as cardiac muscles (Wittenberg et al., 1975). It has recently been reported that myoglobin may also function in detoxification of NO (Flogel et al., 2001).

Neuroglobin (NGB) is expressed in the human and mouse brain and shares little sequence homology with $\mathrm{Hbs}$ and $\mathrm{Mb}$. NGB is believed to represent a distinct globin protein family that diverged early in evolution. However, NGB displays oxygen-binding properties comparable 
to those of myoglobin. This led to the suggestion that neuroglobins enhance oxygen supply in the brain (Burmester et al., 2000).

Cytoglobin (CYGB) is the most recently found globin type protein and is ubiquitously expressed in vertebrate tissues (Burmester et al., 2002). First findings concerning the physiological function indicate a role in collagen synthesis and tissue-protective functions after hypoxic insults (Schmidt et al., 2004; Fordel et al., 2004). Interestingly, CYGB localizes to the nucleus and this suggested a possible function of globin-folded proteins as transcriptional regulators (Geuens et al., 2003).

\subsubsection{The globin gene locus, transcriptional regulation, and mRNA stability}

High-level differential expression within the globin gene clusters during erythroid development and formation of functional hemoglobin requires precise regulatory mechanisms. These appear to involve a complex interplay of chromatin structure, regulatory DNA sequences, and transcription factors. The promoters of all globin genes share remarkable homology. For instance, all globin promoters contain three major regulatory elements, the TATA, CCAAT, and CACC boxes, which are the target sequences of several ubiquitous transcription factors including TATA binding proteins, CP1, and CAC box binding proteins, respectively (Myers et al., 1986). However, unique patterns have been identified also within the promotor sequences. Especially erythroid-specific transcription factors are likely to determine developmental stage specificity of each promoter.

The human alpha-like globin gene cluster spans about $80 \mathrm{~kb}$ and lies close to the telomer of the short arm of chromosome 16 (Buckle et al., 1988). The globin genes are arranged in order

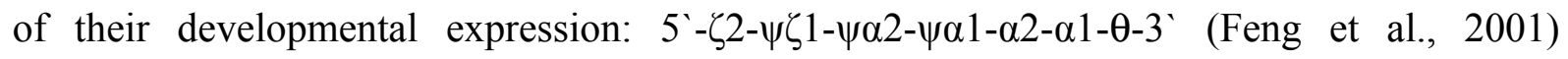
(Figure 3). Sequence analysis found two identical alpha globin genes, $\alpha 1$ and $\alpha 2$, as well as two human alpha globin pseudogenes, $\psi \alpha 1$ and $\psi \alpha 2$, which do not encode for globin peptides (Proudfoot and Maniatis, 1980; Hardison et al., 1986). Differently from the human alpha-like gene cluster, the mouse gene cluster is located internally on chromosome 11 . The arrangement of the mouse alpha-like gene reflects the temporal order of expression as observed for the human genes. The detailed organization of the globin genes, however, varies between human and mouse. For example, there is only a single $\zeta$ gene in mouse. However, in mouse the entire $\psi \alpha-\alpha-\theta$ block has been duplicated; in human only the alpha globin gene has been duplicated. Recently, a third $\psi \alpha-\alpha-\theta$ gene set has been identified on mouse chromosome 17 (Tufarelli et al., 2004). This suggested that the duplication occurred after human and mouse diverged from a common ancestor.

The 5' flanking region of the alpha globin gene cluster is GC-rich and associated with unmethylated $\mathrm{CpG}$ (cytosine and guanine connected by a phosphodiester bond) islands. $\mathrm{CpG}$ islands are deficient in histone $\mathrm{H} 1$, contain hyperacetylated histones $\mathrm{H} 3$ and $\mathrm{H} 4$, and include nucleosome-free DNA segments indicating an open accessible chromatin structure (euchromatin) for constitutive high-level expression of the alpha globin gene. This indicates 
that the alpha globin gene locus displays the characteristics more of a ubiquitously expressed housekeeping gene than a tissue-specific gene (Smith and Higgs, 1999).

Furthermore, human alpha globin expression is regulated by the erythroid-specific DNase I hypersensitive site, HS-40 also known as alpha globin positive regulatory element ( $\alpha$ PRE). HS-40 is the major enhancer and control element of the alpha globin gene family located $40 \mathrm{~kb}$ upstream of the $\zeta$-globin gene (Higgs et al., 1990; Sharpe et al., 1992). The HS-40 core element displays various erythroid-specific and ubiquitous DNA-binding protein sites for GATA, AP1/NF-E2, and CACC binding proteins which behave as enhancers (Loyd et al., 2003).

A comparable element in the mouse alpha globin locus lies $26 \mathrm{~kb}$ upstream of the $\zeta$-globin gene. It is therefore called HS-26 and is thought to have similar functional properties as the human HS-40. In contrast to deletions of human HS-40 which resulted in severe downregulation of alpha globin gene expression, deletion of mouse HS-26 exhibited only mild reduction indicating important differences in human and mouse alpha globin regulation (Anguita et al., 2002). In general, HSs are essential regions for DNA-protein interactions and modeling of the chromatine structure. HSs are believed to facilitate access of regulators and to lower the threshold for activation of linked genes.

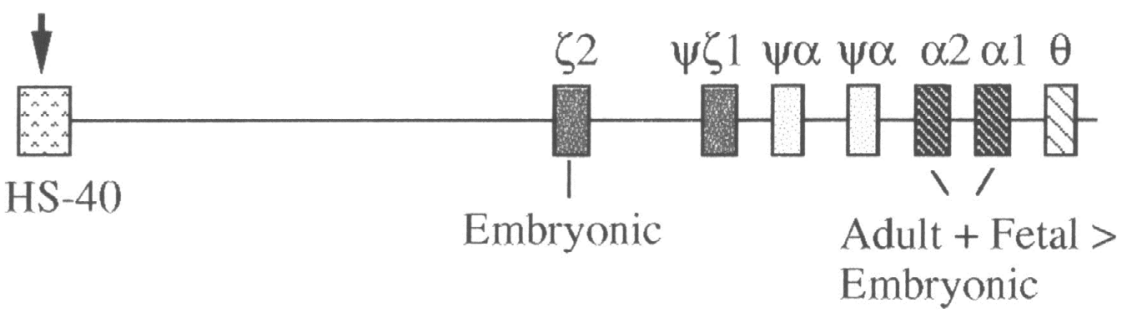

Figure 3: Organization and distal regulatory element of the human alpha globin gene cluster (redrawn from (Hardison, 1998)).

Similar to the alpha globin locus, the genes encoding the beta- and beta-like subunits are present in a cluster in the genome and are arranged in the order they are expressed during development in mammals. For example, the human beta globin gene cluster is organized in the following order: $5^{`}-\varepsilon-\mathrm{G} \gamma-A \gamma-\psi \eta-\delta-\beta-3^{`}$ (Figure 4). In contrast to the human beta globin gene cluster which contains only one sequence for beta globin, the mouse cluster contains two separate sequences, beta globin major (Hbb-b1) and beta globin minor (Hbb-b2). Hbb-b1 and Hbb-b2 share high nucleotide (96\%) and protein (93\%) sequence homology. Both genes are closely linked and coordinately expressed (Konkel et al., 1979). The mammalian DNA sequence in the 5 region of the cluster includes four erythroid-specific DNase I hypersensitive sites, HS-1 to HS-4 which together are referred to as the locus control region (LCR), and a further upstream site, HS-5 (Curtin et al., 1989), which act to open the chromatin in erythroid cells. While chromatin opening does not appear to play a role in regulation of alpha globin genes, it is the key initial step in the regulation of beta globin genes. The HS-5 is ubiquitously and constitutively on, whereas HS-1 to HS-4 are necessary to 
reach full activity. Similar to HS-40 in the alpha globin gene locus, each HS of the LCR exhibits binding sites for transcription factors AP-1/NF-E2, Sp-1, and GATA-1 (Lowrey et al., 1992).

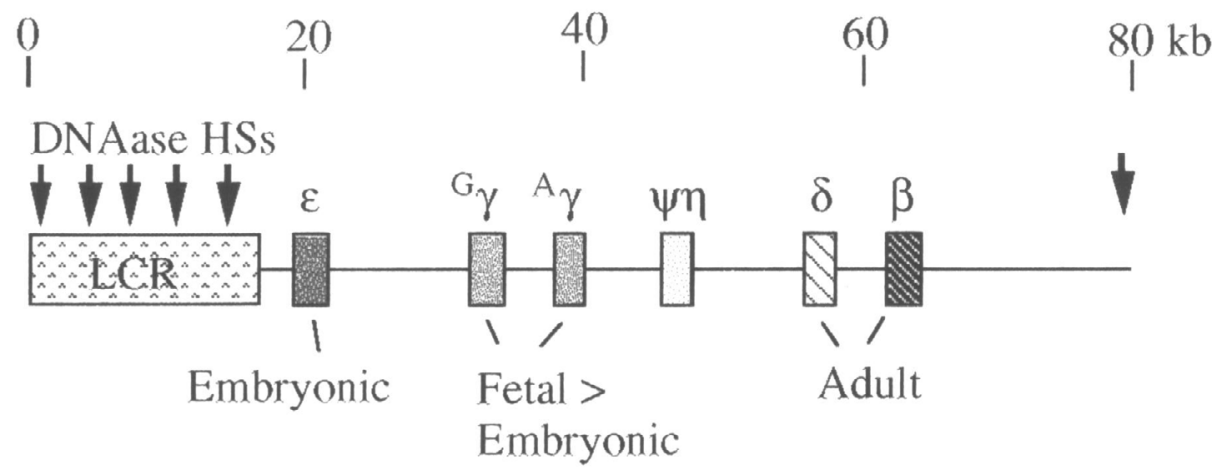

Figure 4: Organization and distal regulatory element of the human beta globin gene cluster (redrawn from (Hardison, 1998)).

The alpha globin and globin mRNAs in general belong to the most stable mRNAs with estimated half-lives ranging from 24-40 h (Volloch and Housman, 1981; Ross and Sullivan, 1985). The major regulatory sequences within the alpha globin gene are three C-rich elements (CRE) located in the 3'UTR at nucleotide positions 25-31, 37-40, and 55-66 from the stop codon (Weiss and Liebhaber, 1995). An RNA-protein complex, called alpha-complex ( $\alpha$-complex) assembles at the minimal $\alpha$-complex binding site, the alpha RNAmin ( $\alpha$ RNAmin), within the CRE of the alpha globin 3'UTR (Wang et al., 1995). Binding of the $\alpha$-complex protects alpha globin mRNA from cleavage by erythroid-enriched endoribonuclease (ErEN) (Rodgers et al., 2002). Alpha globin poly (C)-binding protein 1 and 2 $(\alpha-\mathrm{CP} 1, \alpha-\mathrm{CP} 2)$ were the first two proteins identified in the $\alpha$-complex. Since then additional proteins have been found to associate with this complex: poly-A binding protein (PABP), AU-rich destabilizing elements binding/degradation factor (AUF-1) and two so far unidentified proteins. PABP binds to the poly-A tail of alpha globin and thereby prevents deadenylation and subsequent degradation of alpha globin mRNA (Wang and Kiledjian, $2000)$. Binding of $\alpha-\mathrm{CP}$ to PABP promotes both the binding of $\alpha-\mathrm{CP}$ to $\alpha \mathrm{RNAmin}$ and binding of PABP to the poly-A tail. In the absence of $\alpha-C P$ and PABP, ErEN has access to the ErEN site and cleaves the RNA. So far it is not known whether these two pathways function independently from one another or whether they are functionally linked. Further, the factors that degrade the mRNA after ErEN cleavage are not elucidated.

\subsubsection{GATA transcription factor family}

Binding sites for numerous transcription factors including Sp1 (Pondel et al., 1995), AP-1/NF-E2 (Loyd et al., 2003) (Martin et al., 1998), CP-2 (Lim et al., 1992), NF I (Zorbas et al., 1992), and the GATA transcription factor family have been found in promotor and enhancer elements of the alpha-like globin genes in erythroid cells. Some of these 
transcription factors such as Sp1, NF-E2 or the GATA factors have also been shown to regulate the expression of the beta-like globin genes. Besides shared/common transcription factors, there are transcriptional regulators that seem to exhibit preferences for the beta gene locus, for instance EKLF (Spadaccini et al., 1998) or FOG-1 (Tsang et al., 1997).

GATA factors also known as GF-1, NF-E1, or Eryf 1 have been identified as key molecules in globin gene regulation during erythroid cell differentiation (Tsai et al., 1989). They contain two zinc fingers that recognize and bind to the DNA cis-element consensus motif, (A/T)GATA(A/G). Six different GATA members have been identified in vertebrates so far and can be divided into two subfamilies (Yamamoto et al., 1990). While GATA-4, -5, and -6 are expressed in various mesoderm- and endoderm-derived tissues such as heart, gut (Laverriere et al., 1994), liver, and gonad, where they regulate tissue-specific gene expression (Molkentin, 2000), GATA-1, -2, and -3 exhibit unique but overlapping patterns of expression in hematopoietic tissues (Weiss and Orkin, 1995a). GATA binding sites of the latter subfamily have been initially found in globin gene promoters and enhancers but later in cisregulatory elements of virtually all erythroid-cell-expressed genes (Orkin, 1992). GATA-1 is abundantly and exclusively expressed in erythroid, eosinophilic, mast, and megakaryocytic lineages and multipotential progenitors. It is required for survival of erythroid progenitors and terminal differentiation of erythroid precursors into red blood cells and for maturation of megakaryocytes to platelets. The second member, GATA-2, is present in early hematopoietic progenitors, mast cells, and megakaryocytes, and is crucial for the maintenance and proliferation of immature hematopoietic progenitors (Ohneda and Yamamoto, 2002). GATA-2 is additionally expressed in a limited subset of nonhematopoietic tissues (Yamamoto et al., 1990). GATA-3 is abundantly expressed in T lymphocytes and the brain (Ting et al., 1996).

GATA-1 has structural domains, that enable it to self-associate as well as to heterodimerize with other activators and transcription factors including multitype zinc finger protein FOG (Tsang et al., 1997), Krueppel family proteins Sp-1 and EKLF (Merika and Orkin, 1995), NF-E2 (Andrews, 1998) or CBP/p300 (Blobel et al., 1998). These interacting domains, however, were found to be less conserved in GATA-2 (Orkin, 1992). Closer examination revealed a cross-regulatory mechanism by which GATA-1 can control the expression of GATA-2 and vice versa, possibly via essential GATA binding sites in their cis-acting elements (Ohneda and Yamamoto, 2002; Crossley et al., 1995). Based on these observations and the expression profile in erythroid cells, a model emerged in which GATA-2 activates GATA-1 in the early stage of erythroid differentiation and GATA-1 replaces GATA-2 to promote an autoregulatory loop (Orkin, 1995) (Figure 5). Based on this theory GATA-1 is believed to be the critical protein for erythroid gene transactivation in erythroid cells. However, GATA-1 knock out experiments have shown that GATA-2 could successfully substitute functions believed to be exclusive for GATA-1 (Weiss and Orkin, 1995b). In summary, GATA-1 and GATA-2 are believed to transactivate similar sets of genes, where one or the other is in control depending on the developmental stage as well as the cellular context. 


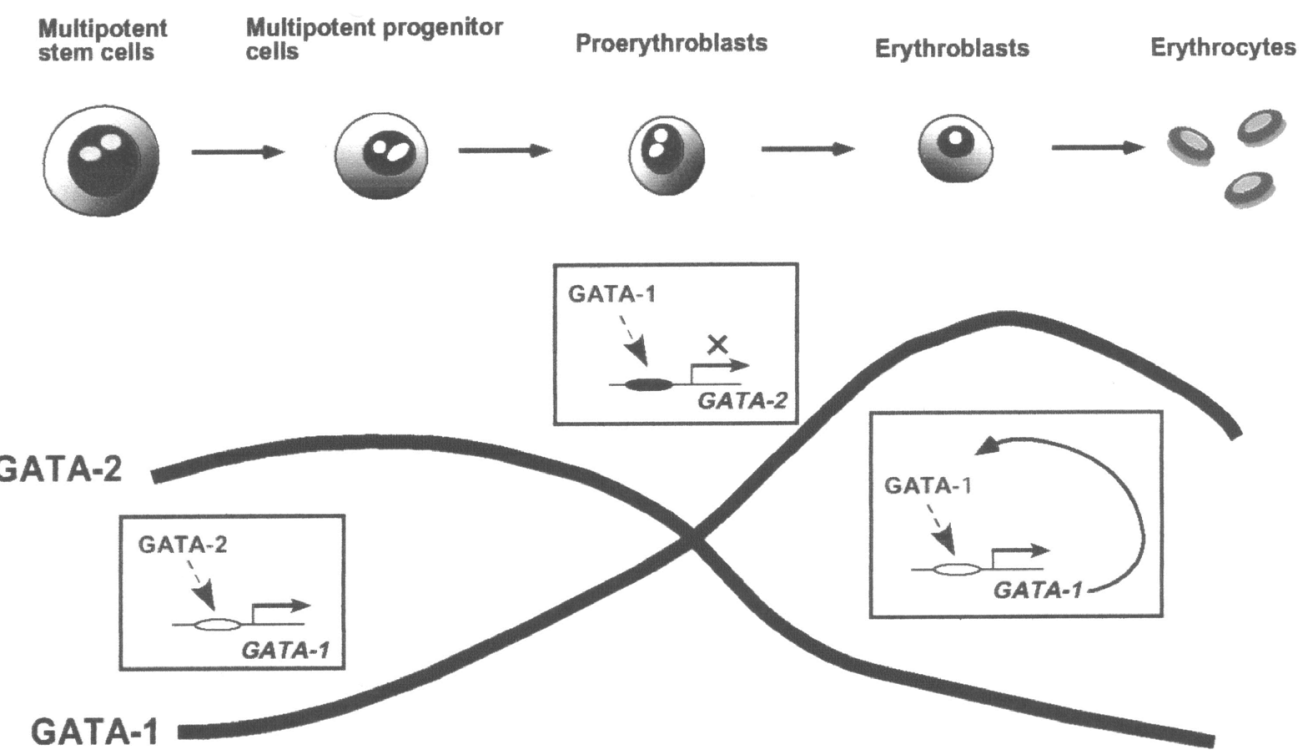

Figure 5: Expression levels of GATA-1 and GATA-2 during erythroid cell development with potential auto- and cross-regulatory effects. In this model, GATA-2 transactivates GATA-1 gene expression, while GATA-1 represses GATA-2 expression. GATA-1 has been shown to induce its own gene expression (redrawn from (Ohneda and Yamamoto, 2002)).

\subsubsection{Assembly of hemoglobin and globin diseases}

Under physiological, healthy conditions the translation of globins into their respective polypeptide chains is followed by the assembly into $\alpha \beta$-dimers. This is known to be the ratelimiting step in hemoglobin assembly (Adachi et al., 2002) and to be driven by electrostatic attraction of the negatively charged beta chain (Adachi et al., 1998). Further dimerization into $\alpha_{2} \beta_{2}$ tetramer results in functional adult $\mathrm{HbA}$.

Expression of mutated, nonfunctional globin polypeptides lead to severe disorders such as sickle cell anemia (Lewis et al., 1966) or thalassemia (Bank et al., 1969; Ingram, 1989). In the "classical" thalassemia, the beta-thalassemia, excess of alpha chains, which cannot build heterotetramers with beta chains, precipitate and form inclusion bodies early during differentiation of young nucleated erythroid cells in the bone marrow (Fessas et al., 1966; E.Rachmilewitz and St.Schrier, 2001). Alpha globin and its oxidized forms, methemoglobin and hemochrome, increase membrane rigidity and apoptosis in pathologic erythrocytes (Fortier et al., 1988; Advani et al., 1992). Under these pathologic conditions alpha globin has been found to form complexes with spectrin and its membrane attachment protein, ankyrin (Kannan et al., 1988). These complexes are believed to be responsible for membrane instability and hemolysis of red blood cells.

\subsubsection{Heme and globin detoxifying proteins, HO-1 and AHSP}

Heme oxygenase 1 (HO-1) also called heat shock protein Hsp32, exerts important functions in the context of hemoglobin degradation and prevention of cellular damage. Heme oxygenase 
exists in three isoforms. HO-1 is dramatically induced upon cellular stress caused by agents such as free heme, heavy metals, UV, low oxygen levels, heat (Shibahara et al., 1987) or antiinflammatory factors such as IL-6 and IL-10 (Ricchetti et al., 2004) and it remains high for hours (Ferris et al., 1999). HO-2, however, is constitutively expressed and has significant heme oxygenase activity (Ewing and Maines, 1991). HO-3 is closely related to HO-2 but is a poor heme oxygenation catalyst (McCoubrey, Jr. et al., 1997). HO-1 is a microsomal enzyme and contains a hydrophobic segment at its $\mathrm{C}$-terminus that is essential for anchorage to the membrane of microsomes. Heme is catabolized in a so called "substrate-assisted" reaction in which it serves as both cofactor and substrate (Schuller et al., 1999). In concert with NADPH, oxygen, and cytochrome P450 reductase, heme oxidizes to biliverdin, ferrous iron, and carbon monoxide (CO). Biliverdin reductase in turn converts biliverdin to bilirubin. Through the Fenton reaction $\mathrm{Fe}^{2+}$ promotes the generation of hydroxyl radicals that damage proteins and lipids. This prooxidant action is limited by ferrous ion inducing the iron chelator, ferritin, as well as by iron pumps that release $\mathrm{Fe}^{2+}$ in the extracellular space (Ferris et al., 1999). CO is a signaling molecule with a wide spectrum of biological functions in neurons, smooth muscle cells, platelets, macrophages, and endothelial cells. $\mathrm{CO}$ has been reported to promote vasodilatation, to inhibit inflammation and to suppress apoptosis (Brouard et al., 2000).

Recently alpha globin stabilizing protein (AHSP) has been described to form stable complexes with alpha but not beta globin independently of heme. AHSP prevents precipitation of alpha globin and oxidation of the heme-bound iron (Kihm et al., 2002). AHSP also known as erythroid association factor (ERAF) is an erythroid-specific, 104 amino acids long protein, expressed in all fetal and adult hematopoietic tissues i.e. bone marrow, spleen, and fetal liver (Gell et al., 2002). Erythroid transcription factor GATA-1 has been shown to strongly upregulate AHSP. In a competitive reaction the alpha globin:AHSP complex is immediately disrupted when beta globin is added to form $\mathrm{HbA}$ (Kihm et al., 2002). Previously AHSP has already been described as erythroid differentiation-related factor (EDRF) and has been found to be transcriptionally downregulated in spleens of animals with transmissible spongiform encephalopathie like Scrapie and bovine spongiform encephalopathy (BSE) (Miele et al., 2001).

\subsubsection{Recent findings on novel functions of alpha globin}

During the past few years, alpha globin has been reported to be involved in processes other than oxygen transport in erythrocytes. Very recently Wride and collegues reported that alpha globin is expressed in lens fibres in normal lenses and is downregulated in the pre-cataractous lens. Lenses undergo an apoptosis-like process during development, which results in removal of organelles and nuclei from lens fibre cells. In cataractous lenses the organelles are not properly removed. This suggests a role of alpha globin in lens fibre differentiation (Wride et al., 2003).

Three years ago Yoshida and colleagues found that extraerythrocytic hemoglobin played a novel role in wound healing. Hemoglobin and globin but not heme or protophorphyrin IX were able to stimulate plasminogen activator biosynthesis and to increase fibrinolytic activity in human fibroblasts (Yoshida et al., 2001). 
We have previously performed gene expression profiling of FL5.12 cells upon induction of apoptosis using cDNA microarrays (Brachat et al., 2000). In FL5.12 cells undergoing apoptosis in the absence of IL-3 alpha globin was the most prominent upregulated gene. We further found alpha globin to be slightly upregulated in FL5.12 cells treated with cisplatin and methotrexate but not staurosporine, camptothecin, or paclitaxel. Devireddy and co-workers independently reported the dramatic increase of alpha but not beta globin in FL5.12 cells after IL-3 deprivation (Devireddy et al., 2001). FL5.12 cells retrovirally transduced with N-terminal FLAG-tagged alpha globin displayed accelerated apoptosis progression and increased caspase-3 activity (Brachat et al., 2002). The pro-apoptotic effect of FLAG-alpha globin was significantly prevented in an FL5.12 cell line stably transfected with Bcl-2. However, the death accelerating effects of FLAG-alpha globin could not be consistently reproduced, nor FLAG-alpha globin expression confirmed on transcript or protein level by RT QPCR or Western blot analysis, respectively. 


\section{Aims of the thesis}

It is now more than 30 year ago that Kerr and Currie described a common type of programmed cell death that the authors repeatedly observed in various tissues and cell types and finally termed apoptosis (Kerr et al., 1972). The authors observed that these dying cells shared many morphological features, which were different from features seen in cells dying of necrosis. They suggested that the shared morphological characteristics might underlie a common, conserved endogenous cell death program. Since then, apoptosis research has evolved to one of the most investigated areas in modern biology. In recent years, central hallmarks of programmed cell death have been characterized and several key players of apoptosis such as the Bcl-2 family members or caspases have been identified. In many pathways, however, regulatory factors and precise interplays within the signaling cascades remain elusive.

In 2000, Brachat et al performed gene expression profiling to explore novel regulators of apoptosis and to shed light on apoptotic signaling pathways in FL5.12 cells. They investigated the transcriptional response in FL5.12 cells upon induction of apoptosis using cDNA microarrays (Brachat et al., 2000). Out of 105 genes induced under cytokine-deprived conditions, the alpha globin gene was found to be most prominently upregulated. Subsequent expression profiling of FL5.12 cells treated with different cytotoxic agents demonstrated, that alpha globin was also upregulated after treatment with cisplatin and methotrexate but not with STS, paclitaxel or campthotecin (Brachat et al., 2002); in summary, alpha globin was differentially regulated depending on the apoptotic stimulus, and appeared to be therefore a novel, promising candidate gene in PCD.

Although cDNA microarrays are powerful tools for analyzing gene expression on a genome scale, the biological relevance of a regulatory event cannot be unequivocally deduced from expression profiling. Therefore, we first attempted to confirm the upregulation of alpha globin transcript in independent and repeated experiments by means of RT QPCR, a method that allows relative quantification of gene expression. Moreover, we wanted to show that the protein was present and regulated similarly to the transcript. To elucidate whether upregulated alpha globin formed hemoglobin, we investigated the expression of beta globin and beta-like globin genes, as well as the heme and hemoglobin contents. By means of both overexpression of ectopic alpha globin and downregulation of endogenous alpha globin we intended to evaluate, whether alpha globin was indeed involved in the apoptotic process or whether upregulation of alpha globin was an unrelated side effect. It was of further interest to ascertain, which cell death pathway was specifically promoted by alpha globin. A closing objective of the current work was to shed light on the regulatory mechanisms leading to elevated alpha globin levels during apoptotic cell death. 



\section{$3 \quad$ Results}

\subsection{Upregulation of alpha globin promotes apoptotic cell} death in the hematopoietic cell line FL5.12

\subsubsection{Alpha globin mRNA is highly expressed and upregulated in cytokine-deprived FL5.12 cells}

Incyte cDNA microarray experiments and Northern blot analyses have shown that alpha globin transcript was upregulated upon cytokine deprivation in FL5.12 cells (Brachat et al., 2000a). We confirmed the results using the Affymetrix chip technology (Figure 1, upper panel).
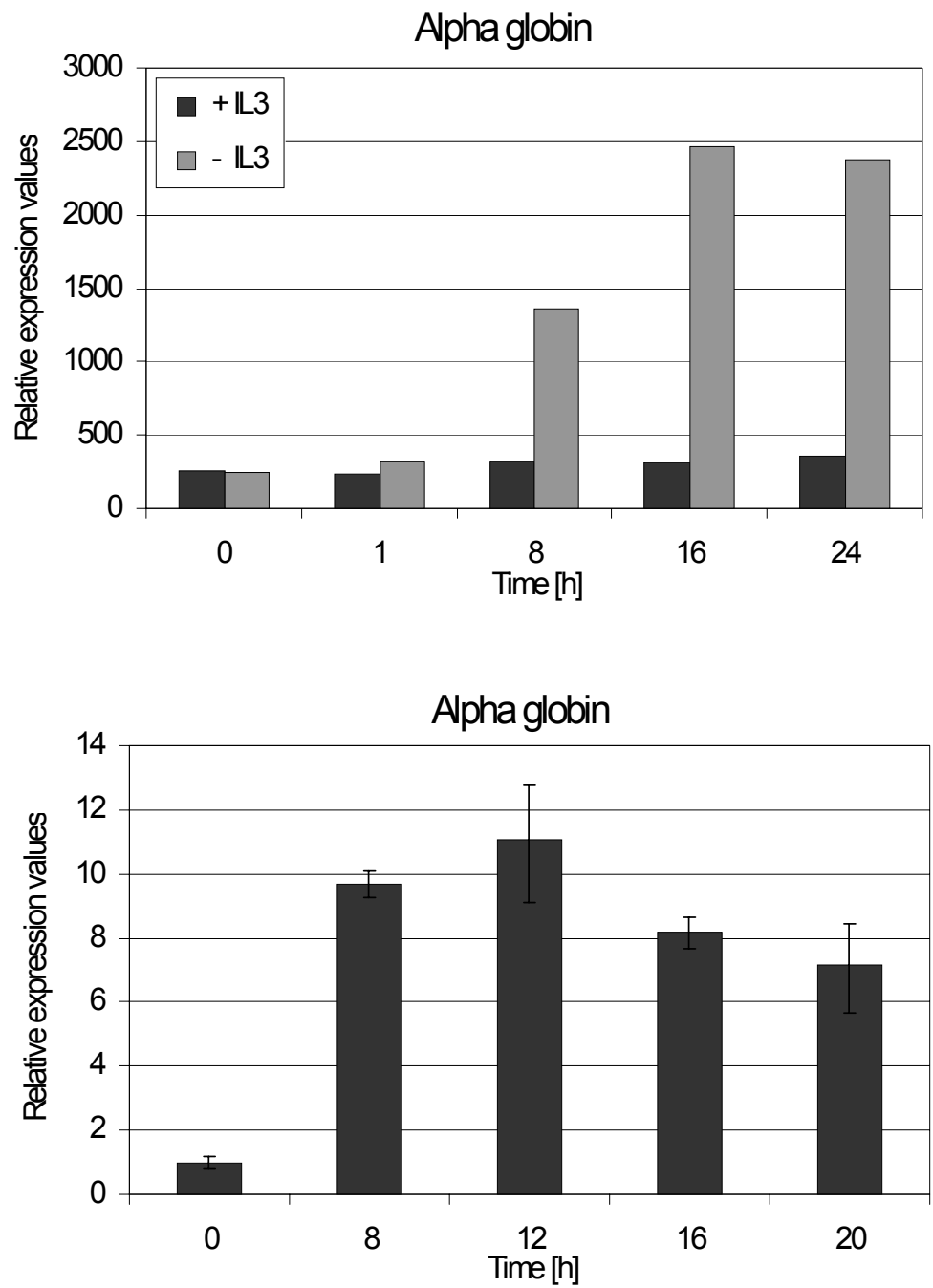

Figure 1: Alpha globin mRNA expression profile of FL5.12 cells growing with or without IL-3. Gene expression analysis for alpha globin RNA samples prepared $0,1,8,16$, and $24 \mathrm{~h}$ after IL-3 removal (upper panel). The graphs represent one of two independent chip experiments. Alpha globin RNA expression levels measured with RT QPCR in FL5.12 cells 0, 8, 12, 16, and $20 \mathrm{~h}$ after IL-3 deprivation (lower panel). Each bar represents the average of triplicate measurements. 
In order to assess basal expression levels of alpha globin in FL5.12 cells and the profile of regulation after IL-3 deprivation, we performed quantitative Real Time RT PCR of FL5.12 cells growing with or without IL-3 for $0,8,12,16$, and $20 \mathrm{~h}$. 18S ribosomal RNA was used as endogenous control to normalize for differences in the amount of total RNA. GAPDH, another frequently used housekeeping gene, was found to be downregulated upon apoptosis induction and was therefore not suitable as a reference in RT QPCR (data not shown). Basal alpha globin expression levels in FL5.12 were relatively high. We measured a threshold cycle of 16 cycles for the housekeeping gene, $18 \mathrm{~S}$ ribosomal RNA, compared to 22 cycles for alpha globin transcript. The largest incremental increase of alpha globin transcript of about 9.7-fold was observed within the first $8 \mathrm{~h}$ after IL-3 removal. Maximal expression of alpha globin (11fold induction) was reached after $12 \mathrm{~h}$ and then followed by a decrease 16 and $20 \mathrm{~h}$ after IL-3 deprivation (Figure 1, lower panel).

\subsubsection{Other globins or globin-like genes are expressed at low or undetectable levels}

To check if other globin genes were expressed and regulated under death-inducing conditions, we analyzed expression patterns from chip experiments of IL-3-deprived FL5.12 and control FL5.12 cells. Transcript for myoglobin, a globin protein known to function as an oxygen carrier in muscles, heart and other tissues was not detectable in FL5.12 cells. Probes for neuroglobin, the oxygen carrier in the brain (Burmester et al., 2000) or cytoglobin, the most recently found globin (Burmester et al., 2002), were not spotted on the chip and were not further investigated. Additionally, neither zeta nor epsilon or gamma globin resembling the alpha-and beta-like globins and expressed in early embryonic life were detectable. Beta globin was not detected in FL5.12 cells as assessed by gene chip analysis. However, after cytokine removal the signal for beta globin was slightly increased, but according to Affymetrix chip analysis beta globin was still termed absent (Figure 2, upper panel). To confirm the chip results we performed RT QPCR using beta globin-specific primers and probe. In mouse, two copies of the beta globin gene exist, beta globin major (Hbb-b1) and minor chain (Hbb-b2), which share $96 \%$ homology at nucleotide level. Neither gene chip arrays nor RT QPCR could discriminate between beta major and minor chain. The very low expression levels for beta globin were confirmed by RT QPCR. The upregulation of beta globin message upon IL-3 deprivation, however, appeared to be much stronger in RT QPCR than on Affymetrix chips suggesting that RT QPCR is the more sensitive method to detect gene expression (Figure 2, lower panel). For comparison, K562, a human chronic myeloid leukemia cell line, was analyzed. K562 cells are known to express only beta globin-like genes, epsilon and gamma globin, but not beta globin itself when forming embryonic and fetal hemoglobin (Rutherford et al., 1981). In RT QPCR experiments, the detection threshold for beta globin in K562 cells was reached after 30 cycles similar to our results for beta globin in FL5.12 cells. We therefore assume that beta globin expression in FL5.12 cells is negligible. 

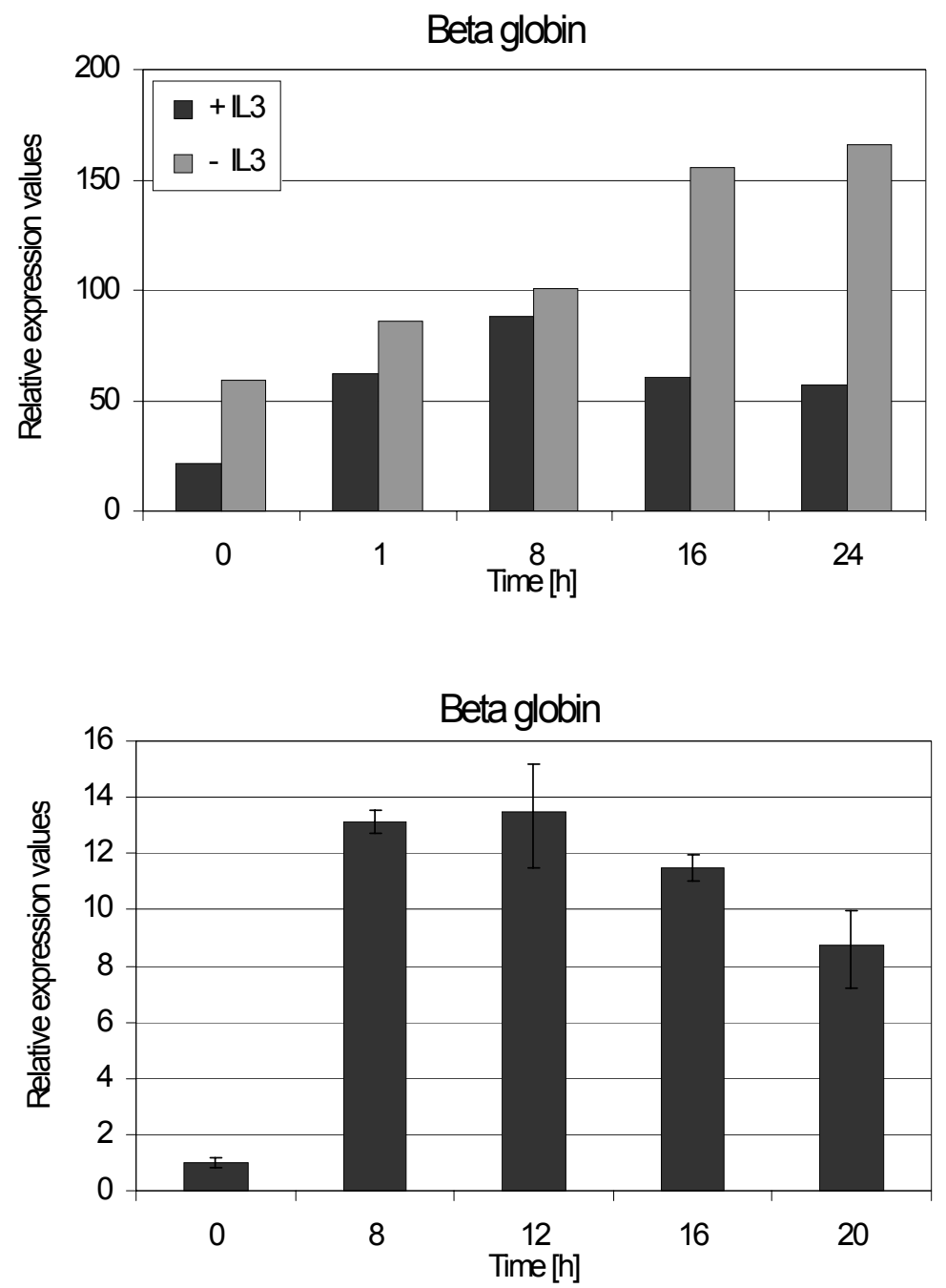

Figure 2: Beta globin mRNA expression profile of FL5.12 cells growing with and without IL-3. Gene chip expression analyses for beta globin RNA samples prepared $0,1,8,16$, and $24 \mathrm{~h}$ after IL-3 removal (upper panel). The graphs represent one of two independent gene chip experiments. Beta globin RNA expression levels measured with RT QPCR in FL5.12 cells 0, 8, 12, 16, and $20 \mathrm{~h}$ after IL-3 deprivation (lower panel). Each bar represents the average of triplicate measurements.

\subsubsection{Alpha globin protein is upregulated upon IL-3 deprivation}

In order to show that alpha globin protein was regulated similarly to its mRNA, we performed Western blot analysis and immunostaining with a hemoglobin antibody. First we tested the specificity of the hemoglobin antibody against alpha and beta globin. We transiently transfected HeLa cells with mouse GFP-alpha globin and GFP-Hbb-b1 and examined protein expression using an anti-GFP and the anti-hemoglobin antibodies in parallel blots. We ascertained that the hemoglobin antibody recognized both globin chains (Figure 3a, b). In general we found the hemoglobin antibody to be unspecific as it detected several unspecific background bands. Unfortunately, this was the only functional antibody, which was 
commercially available, while all other antibodies against hemoglobin tested failed to detect alpha globin.

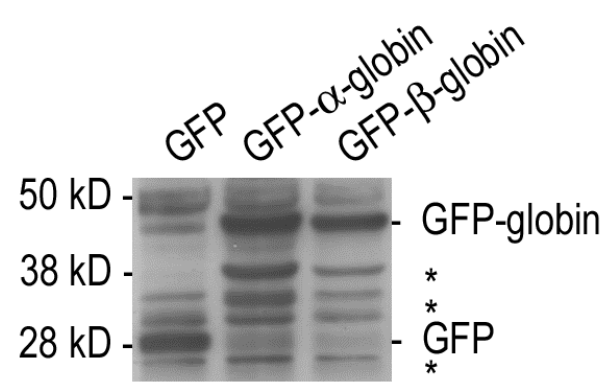

a

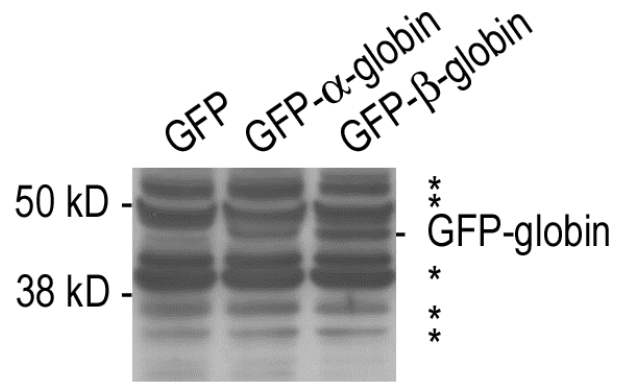

$b$
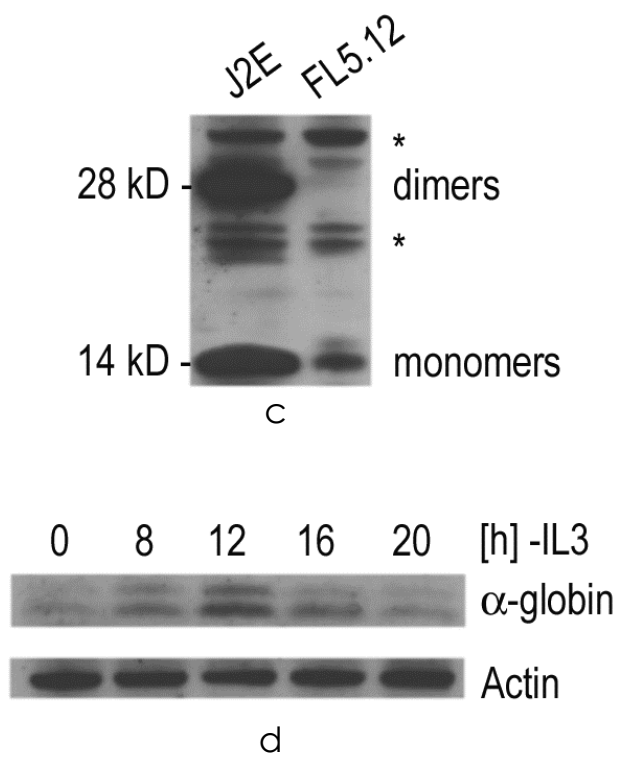

Figure 3: Alpha globin protein is upregulated upon IL-3 deprivation in FL5.12 cells and appears as a double band. Whole cell lysates of HeLa cells transfected with GFP, GFP-alpha globin or GFP-Hbb-b1 (a, b) served to evaluate the specificity of the hemoglobin antibody. Whole cell lysates of J2E control and FL5.12 cells (c) and FL5.12 cells growing in the absence of IL-3 for $0,8,12,16$, and $20 \mathrm{~h}$ (d) were prepared. All proteins were analyzed by SDS PAGE and Western blotting. Blots were probed with anti-GFP (a) or anti-hemoglobin antibody (b, c, d). Background bands are marked with asterisks. The $38 \mathrm{kD}$ background band, probably a degradation product of GFPglobin, occurred in HeLa but not FL5.12 cells.

Next we analyzed endogenous globin protein levels in FL5.12 cells growing in the absence of IL-3 for $0,8,12,16$, and $20 \mathrm{~h}$. For the first time we were able to detect endogenous alpha globin at the protein level in FL5.12 cells. We found a comparable expression profile for the protein as seen for the transcript (Figure 3d). Interestingly, we observed two bands with approximately $0.5 \mathrm{kD}$ difference. The intensity of both bands increased upon IL-3 deprivation. We assumed that both protein bands most probably represent alpha globin since 
beta globin mRNA was expressed at its detection limit in FL5.12 cells (compare 4.1.2). Further, the hemoglobin antibody detected a single band for alpha and beta globin deriving from mouse erythroid J2E cells (Figure 3c). The globin monomers in J2E cells corresponded to the smaller $14 \mathrm{kD}$ globin band in FL5.12 cells. The slower migration of the $14.5 \mathrm{kD}$ band could be due to unknown posttranslational, secondary modifications.

Immunostaining of fixed FL5.12 cells was performed to study cellular localization of alpha globin. We demonstrated that alpha globin protein was predominantly expressed in the cytoplasm. However, the expression pattern of alpha globin did not appear to be homogenous but somewhat granular, especially in cells lacking IL-3 (Figure 4). Staining of FL5.12 cells with the secondary antibody, goat anti-rabbit Alexa Fluor 546, alone served as control, from which no background signal was detected (data not shown). 

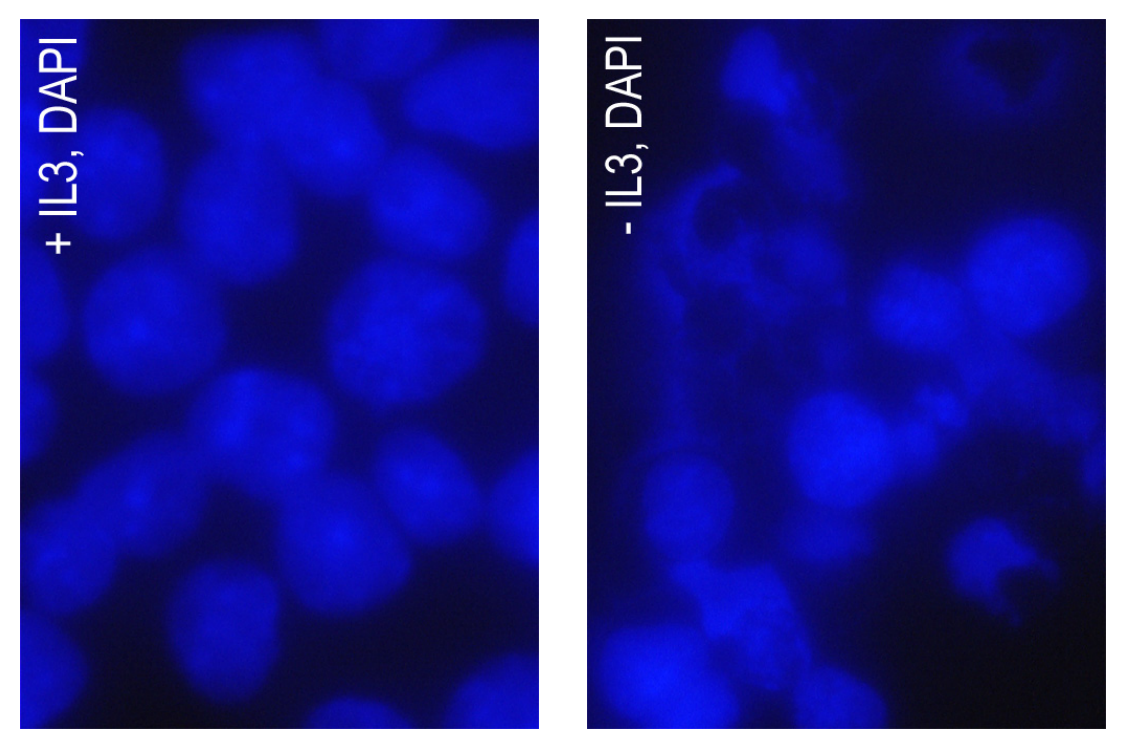

ฮิ

考

on 3

包

क ⿱宀⿻三丨口

ల

그요

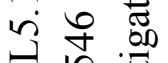

空 n

$\dot{\overline{0}} \stackrel{\dot{0}}{\stackrel{0}{\Xi}}$.
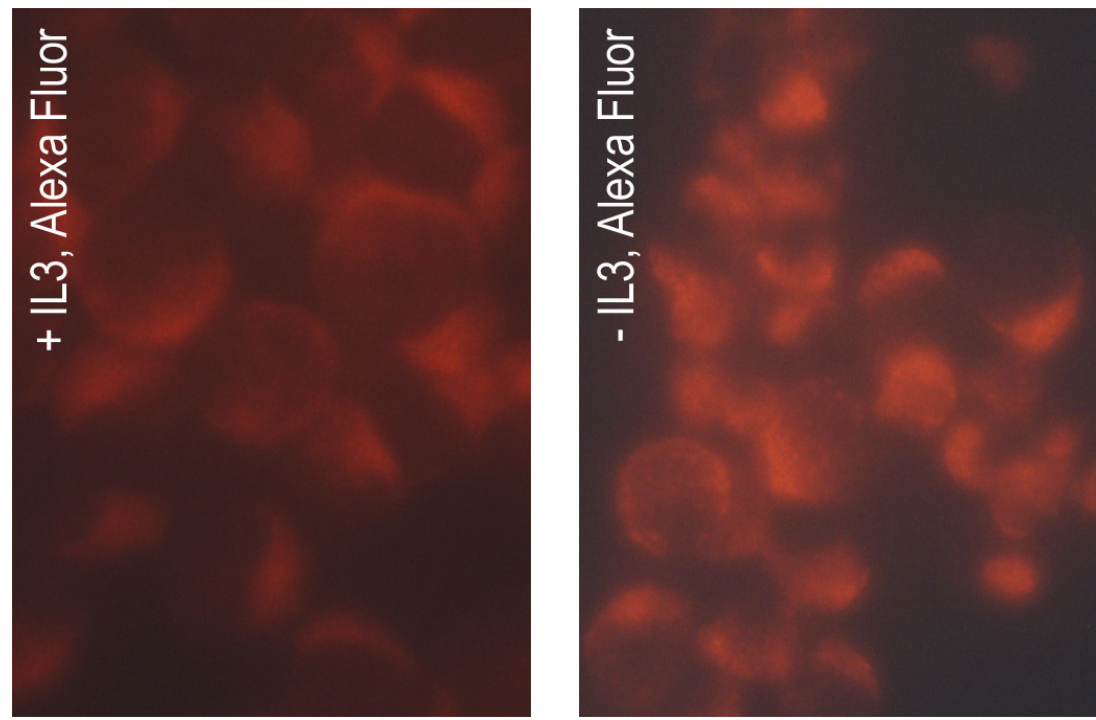

둔

जि

至过它

잉

. 흘

वे क्ष

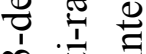

点 音

$\Xi$ 휴

छ

范

टे है

\&

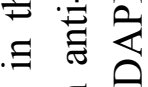
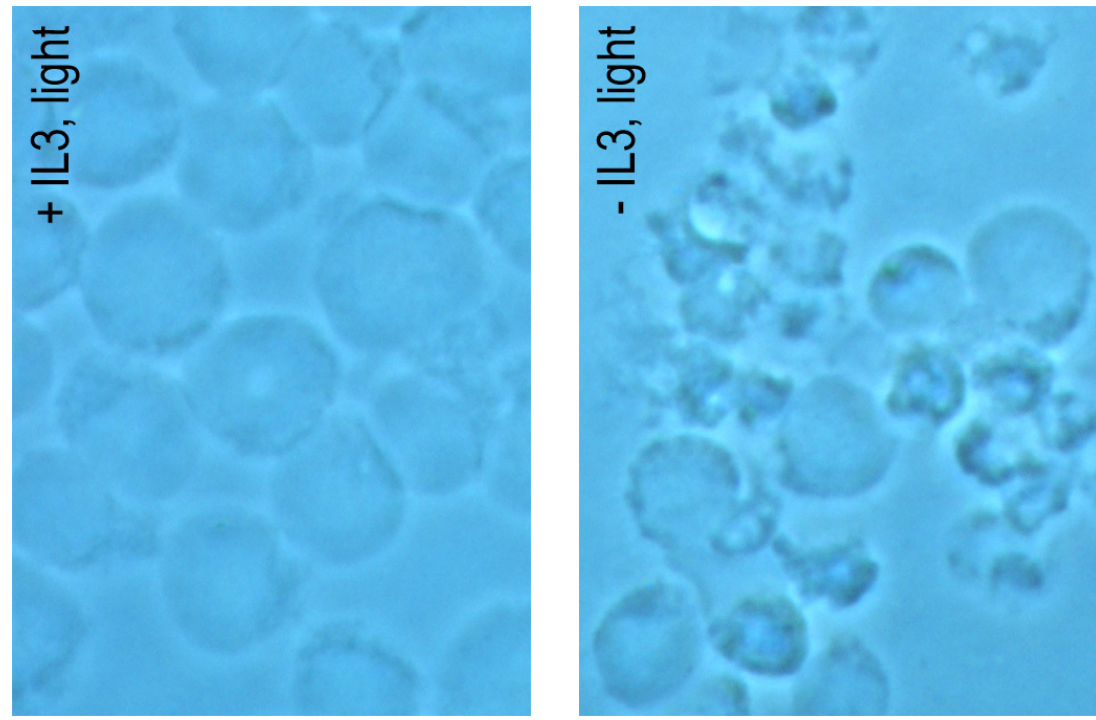

$n=\theta$

兽考

छ

忌 忢

.

ए

t. $=$ 일

$\exists \simeq$ 금

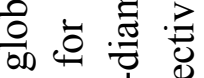

产

यद

$\ddot{\forall}=$ oे

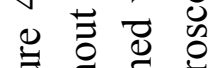

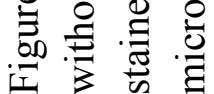




\subsubsection{Alpha globin is localized to the cytoplasm and partly associates with the cytoskeleton after IL-3 deprivation}

To study more closely the localization of alpha globin in FL5.12 cells under normal as well as cytokine-deprived growth conditions we fractionated cells into cytosolic, membrane, nuclear, and cytoskeletal protein fractions. As indicated by immunoblotting, alpha globin was found mainly in the cytoplasm. In apoptotic, but not in healthy cells, the $14 \mathrm{kD}$ alpha globin band appeared also in the cytoskeletal fraction (Figure 5a, b). The cytoskeletal proteins including alpha globin were the last fraction obtained in the fractionation protocol, while the fractions prepared between the cytosolic and the cytoskeletal fractions (membrane, nuclear) lacked alpha globin. We therefore exclude that the appearance of alpha globin in the cytoskeletal fraction reflected merely contamination of the cytoskeletal fraction with cytosolic proteins.

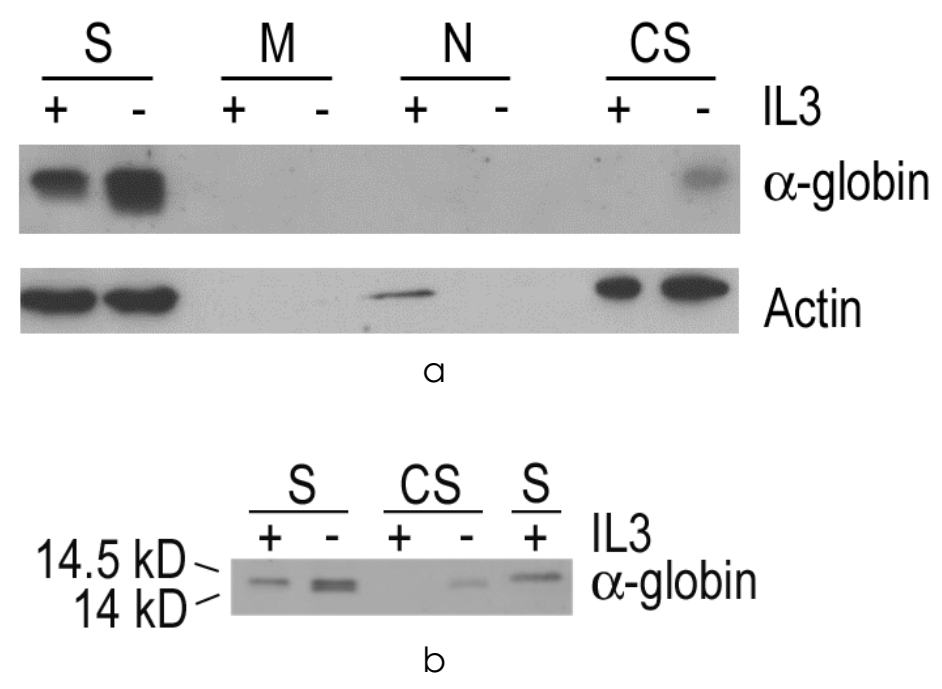

Figure 5: The $14 \mathrm{kD}$ alpha globin appears in the cytoskeletal fraction of FL5.12 cells lacking IL-3. FL5.12 cells were grown with or without IL-3 for $16 \mathrm{~h}$. Cells were fractionated to soluble/cytosolic (S), membrane $(\mathrm{M})$, nuclear $(\mathrm{N})$, and cytoskeletal fractions (CS) using the Proteo Extract kit. Proteins were analyzed by SDS PAGE and Western blotting. The blots were probed with anti-hemoglobin antibody, stripped and reprobed with anti-actin antibody (a), or only probed with anti-hemoglobin antibody (b).

\subsubsection{Differential expression of alpha globin after cisplatin, doxorubicin, staurosporine, and cycloheximide/TNF alpha treatment}

Next, we quantified alpha globin message by RT QPCR after treatment with cytotoxic reagents. We used compounds known to induce cell death along different pathways. Treatment was continued until $50 \%$ of the FL5.12 cells were dead according to trypan blue staining. Both cisplatin and doxorubicin, two DNA-intercalating agents, induced alpha globin 
weakly about 2.4-fold. In comparison, deprivation of IL-3 for $20 \mathrm{~h}$ increased alpha globin message approximately 8-fold (Figure 6, upper panel). The broad-spectrum protein kinase inhibitor, staurosporine (STS) (Bertrand et al., 1994), known to inhibit multiple kinasedependent survival pathways, triggered apoptosis faster than seen in cytokine-deprived cells, but did not change alpha globin levels after 4, 8 or 12 h (Figure 6, middle panel). Last, we examined the TNF alpha death receptor signaling pathway. This pathway triggers apoptosis without de novo transcription and translation (Abreu-Martin et al., 1995). TNF alpha $(2 \mathrm{ng} / \mathrm{ml})$ alone did not induce apoptosis. It has been shown earlier that FL5.12 cells need to be sensitized by translation inhibitor cycloheximide (CHX) to undergo apoptosis by TNF alpha treatment (Johnson and Boise, 1999; Fulda et al., 2000). While treatment with CHX $(1 \mu \mathrm{g} / \mathrm{ml})$ alone did not change the viability either, combined treatment of TNF alpha and CHX was toxic leading to 50\% trypan blue-positive cells after $12 \mathrm{~h}$. Neither TNF alpha nor CHX changed alpha globin transcript. In contrast, combined treatment with CHX/TNF alpha caused a decrease of transcript levels (Figure 6, lower panel).

In summary, alpha globin was most strongly induced in apoptosis triggered by cytokine withdrawal as well as to some extent by DNA-damaging drugs (Johnson and Boise, 1999; Siddik, 2003), but not by death receptor stimulation or protein kinase inhibition (Jiang et al., 1999).
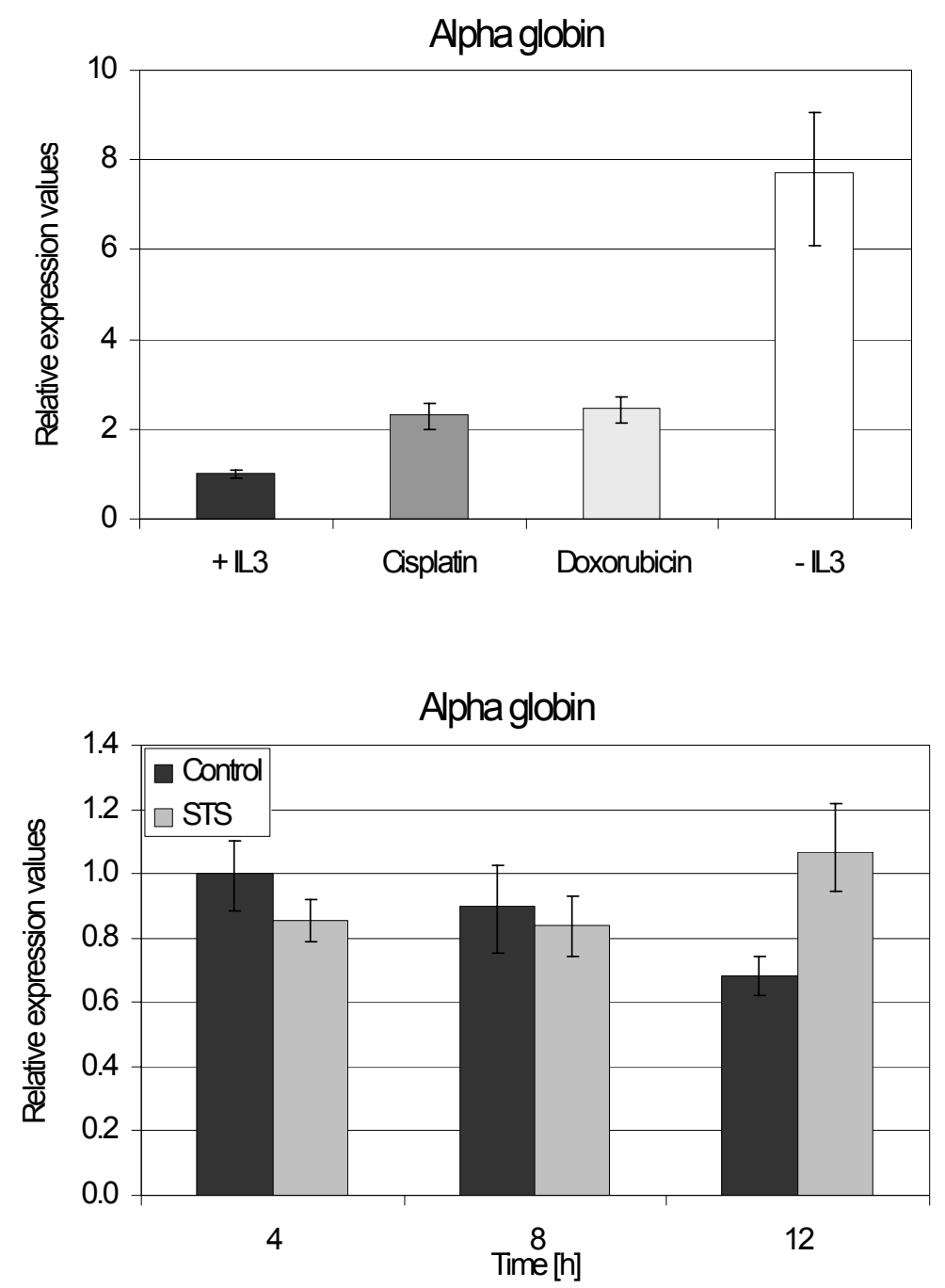


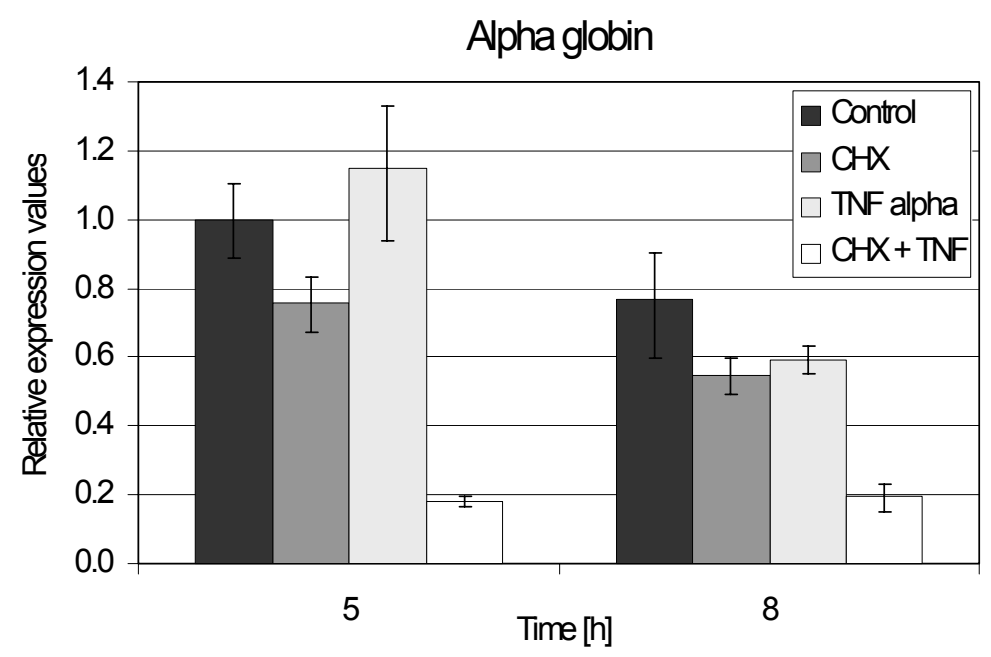

Figure 6: Expression profile of alpha globin mRNA in FL5.12 cells undergoing apoptosis induced by different cytotoxic agents measured with RT QPCR. Total RNAs were isolated from FL5.12 cells treated with $20 \mu \mathrm{M}$ cisplatin, grown without IL-3 or left untreated for $20 \mathrm{~h}$ or treated with $40 \mathrm{nM}$ doxorubicin for $40 \mathrm{~h}$ (upper panel); FL5.12 cells treated with $20 \mathrm{nM}$ STS versus untreated cells (middle panel); FL5.12 cells treated with $1 \mu \mathrm{g} / \mathrm{ml} \mathrm{CHX,} 2 \mathrm{ng} / \mathrm{ml} \mathrm{TNF}$ alpha, with $1 \mu \mathrm{g} / \mathrm{ml}$ CHX plus $2 \mathrm{ng} / \mathrm{ml}$ TNF alpha (CHX + TNF), or left untreated (lower panel).

\subsubsection{Alpha globin is not associated with heme}

We next addressed the question whether alpha globin was associated with heme. We assumed that genes involved in heme biosynthesis should parallel alpha globin expression if alpha globin were associated with heme. Heme, the prosthetic group in globins, coordinately binds iron for oxygen transport (Hardison, 1996). We found that neither aminolevulinic acid synthase 1 (ALA-S1) (Figure 7, upper panel) nor porphobilinogen deaminase (PBGD) (Figure 7, lower panel), two representatives of eight enzymes involved in heme biosynthesis, were upregulated in gene chip experiments.

We conducted two experiments to verify this observation. First, we analyzed expression of heme oxygenase 1 (HO-1). HO-1, also called heat shock protein Hsp32, bears important functions in hemoglobin and especially heme degradation and prevention of cellular damage. HO-1 is dramatically induced upon cellular stress, caused by free heme, heavy metals, UV, low oxygen treatment or heat (Shibahara et al., 1987) and remains high for hours. We assumed that HO-1 would increase under IL-3-deprived conditions if alpha globin were associated with heme and would be degraded during apoptosis. While alpha globin levels increased in FL5.12 cells growing without IL-3, HO-1 remained unchanged. In contrast, cells treated with heme responded with induction of HO-1 (Figure 8, upper panel). Second, we measured cellular heme concentrations as described previously (Sassa, 1976). Detection of heme is based on the conversion of heme to its fluorescent porphyrin derivative by removal of heme iron under acidic reducing conditions. In parallel to FL5.12 cells growing with or 
without IL-3, we analyzed K562 cells that have been reported to have increased globin expression and generate hemoglobin upon treatment with doxorubicin in subtoxic concentrations (Aries et al., 1996). In K562 cells, we found basal heme concentrations which were about 17-fold higher than in FL5.12 cells. Whereas heme levels increased significantly in K562 cells upon doxorubicin treatment, levels remained unchanged in growth factordeprived FL5.12 (Figure 8, lower panel).
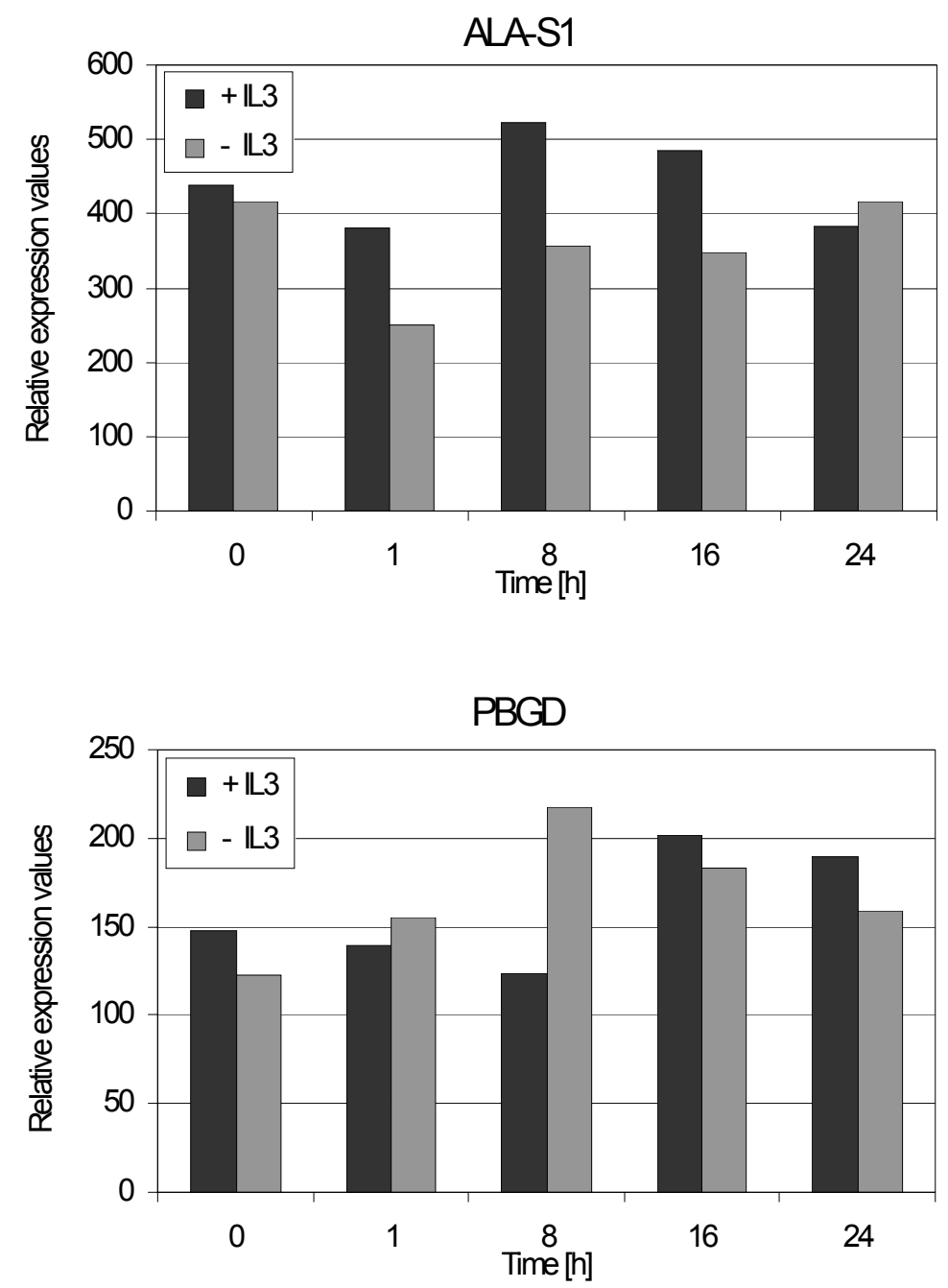

Figure 7: ALA-S1 and PBGD are not upregulated in FL5.12 cells growing without IL-3. Gene chip expression analysis for ALA-S1 (upper panel) and PBGD (lower panel) RNA samples prepared 0, 1, 8, 16 , and $24 \mathrm{~h}$ after IL-3 removal. The graphs represent one of two independent chip experiments. 

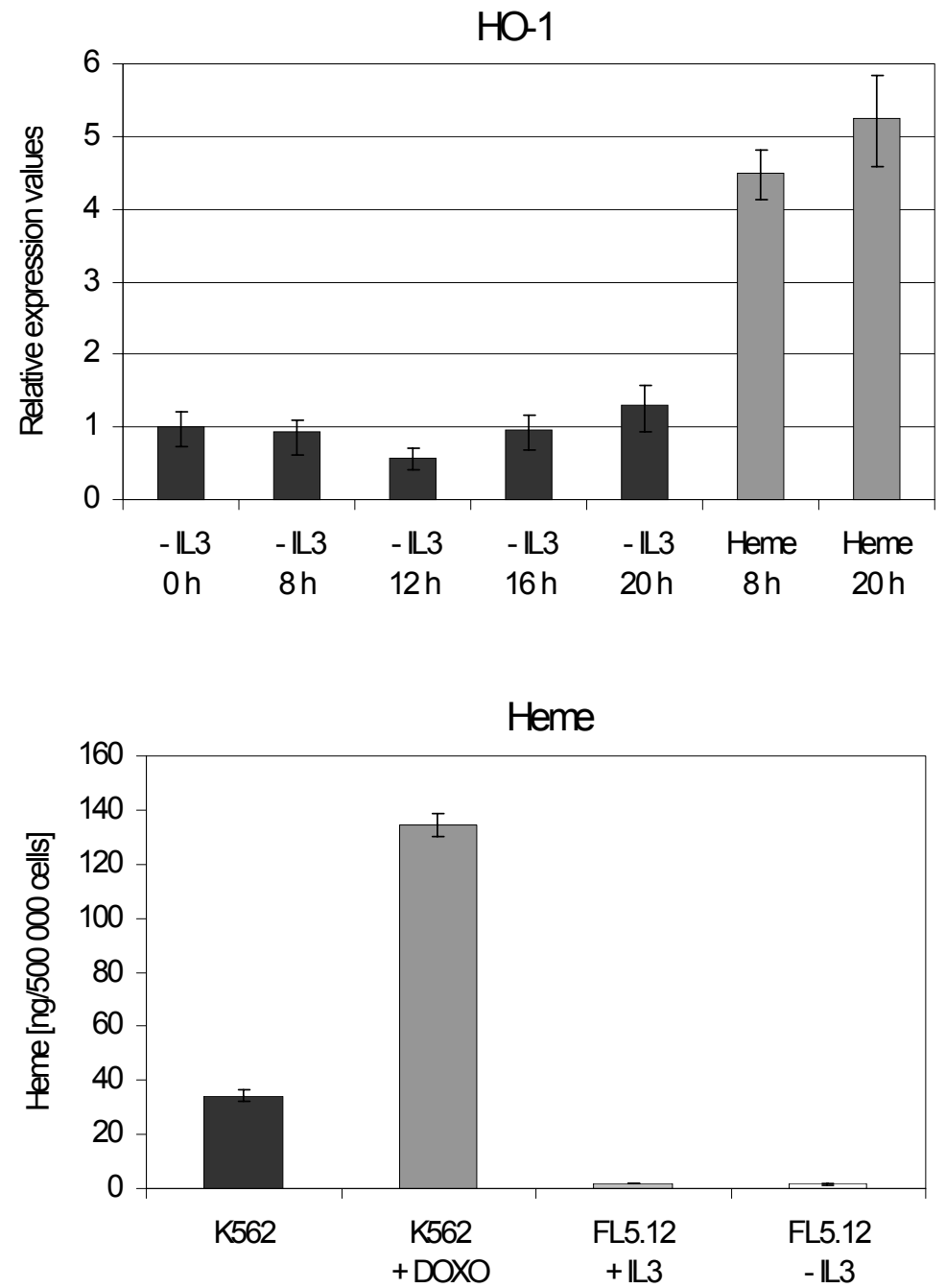

Figure 8: Alpha globin is not associated with heme in FL5.12 cells. HO-1 transcript levels were measured by RT QPCR in FL5.12 cells growing with or without IL-3 for $0,8,12,16$, and $20 \mathrm{~h}$ or treated with $25 \mu \mathrm{M}$ heme for 8 and $20 \mathrm{~h}$ (upper panel). Heme contents were measured in FL5.12 growing with or without IL-3 for $12 \mathrm{~h}$ and K562 cells treated with doxorubicin $(40 \mathrm{nM})$ or left untreated for $60 \mathrm{~h}$ (lower panel). Graphs for $\mathrm{HO}-1$ and heme represent triplicate measurements of three independent experiments.

\subsubsection{Cytokine-deprived FL5.12 cells neither produce hemoglobin nor differentiate down the erythroid lineage}

Next we measured intracellular hemoglobin concentration in K562 and FL5.12 cells using the method of Worthington (Worthington et al., 1987). The hemoglobin assay is based on the oxidation of 2,7-diaminofluorene (DAF) to fluorene blue by the pseudoperoxidase activity of hemoglobin in the presence of peroxide. The DAF staining method was found to reliably measure hemoglobin. Doubling the amount of K562 cells led to a 2-fold increase of fluorene blue. Treatment of K562 cells with doxorubicin increased hemoglobin levels about 9-fold. In 
contrast, hemoglobin was not detected in FL5.12 cells growing with or without IL-3 (Figure 9).

It has been reported that differentiation of K562 cells is further characterized by reduced proliferation and an increase in cell size (Nyoung et al., 1994). Hence, we investigated morphology of K562 and FL5.12 cells after doxorubicin treatment and IL-3 deprivation, respectively (Figure 10a - d). As typical for K562 cells we detected an enormous increase of cell size under treatment with doxorubicin (Figure 10b) but not in control cells (Figure 10a). Differentiation was accompanied by slower proliferation but not cell death. In contrast, apart from a low percentage of cells, which were already committed to apoptosis, FL5.12 cells lacking IL-3 (Figure 10c) did not exhibit morphological changes as compared to cells growing with IL-3 (Figure 10d). This was further confirmed with Eosin G and Thiazine staining which is frequently used to assess the differentiation stage of hematopoietic cells. FL5.12 cells lacking IL-3 displayed moderate cell shrinkage, a decrease in nucleoli, and the occurrence of cell debris (Figure 10f) as compared to control cells (Figure 10e). Apart from the morphological changes typical for cells undergoing apoptosis, no signs of differentiation were detected.

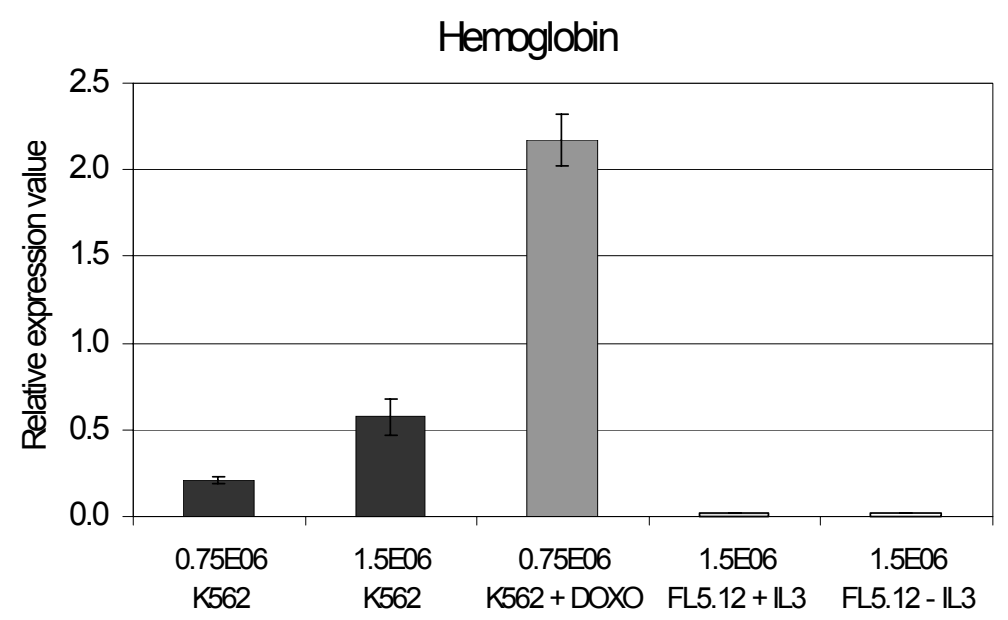

Figure 9: FL5.12 cells do not synthesize hemoglobin upon deprivation of IL-3. Hemoglobin contents were measured of $7.5 \times 10^{5}$ and $1.5 \times$ $10^{6} \mathrm{~K} 562$ control cells, of $7.5 \times 10^{5} \mathrm{~K} 562$ cells treated with doxorubicin $(40 \mathrm{nM})$ for $60 \mathrm{~h}$, and of $1.5 \times 10^{6}$ FL5.12 cells growing with or without IL-3 for $12 \mathrm{~h}$. Each graph represents triplicate measurements of three independent experiments. 
a

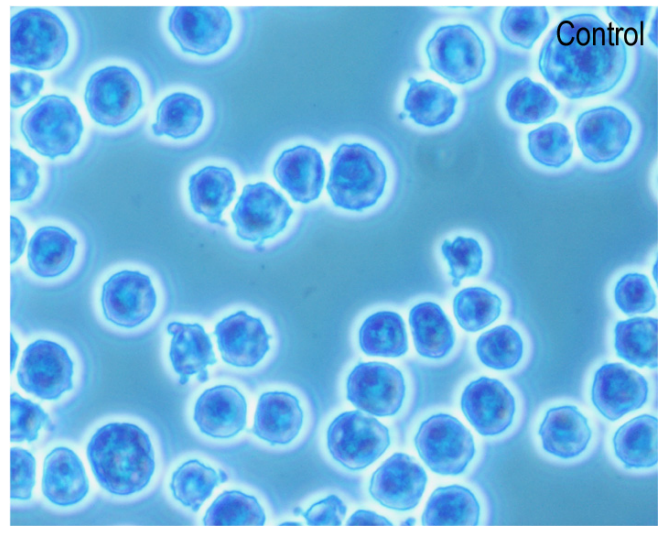

C

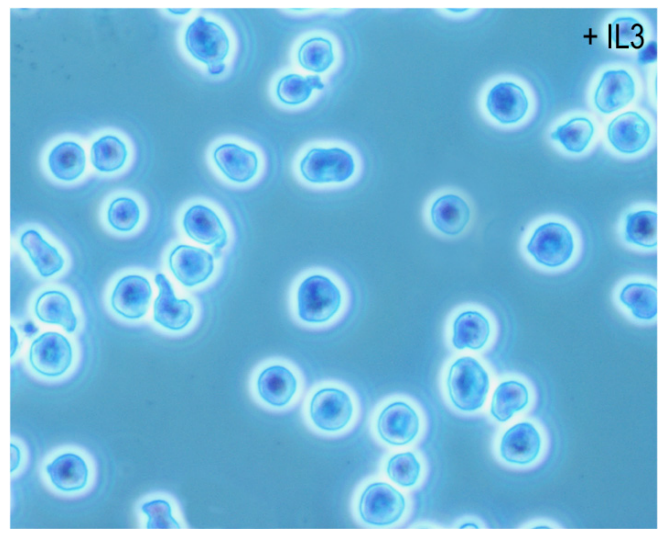

e

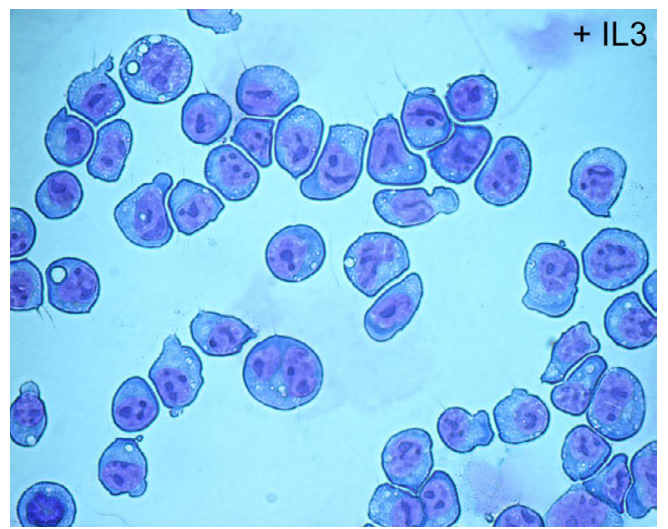

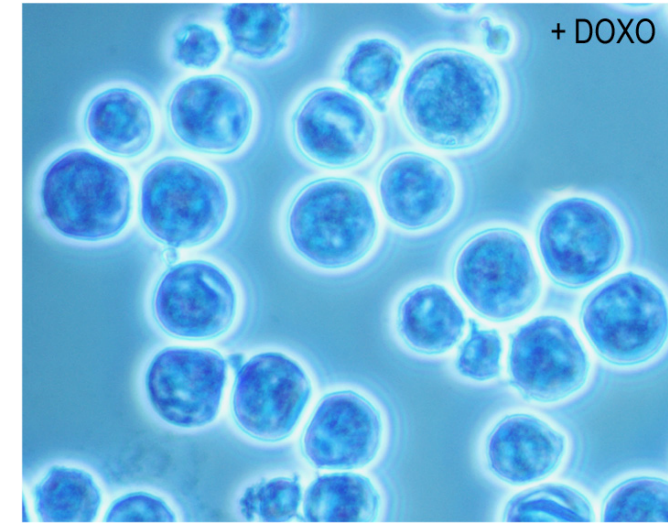
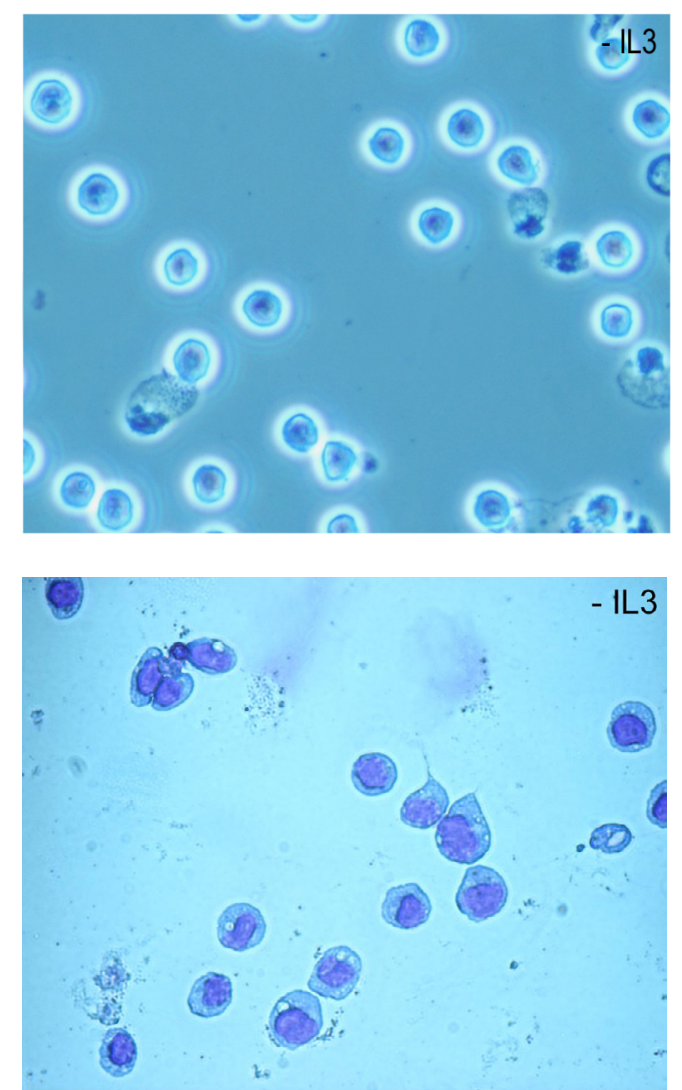

Figure 10: FL5.12 cells do not differentiate down the erythroid lineage upon deprivation of IL-3. K562 cells were treated with $40 \mathrm{nM}$ doxorubicin (b) or left untreated for $60 \mathrm{~h}$ (a), FL5.12 cells were grown with (c) or without IL-3 (d) for $12 \mathrm{~h}$. Cells were investigated with by light microscopy. FL5.12 cells growing with (e) or without IL-3 (f) for $12 \mathrm{~h}$ were further stained with Eosin $\mathrm{G}$ and Thiazine dye and morphology was studied using light microscopy.

\subsubsection{AHSP does not change the viability of FL5.12 cells}

Recently the alpha hemoglobin stabilizing protein, AHSP, has been reported to function as a chaperone of alpha globin. AHSP forms a stable complex with alpha but not beta globin both in vitro and in vivo (Kihm et al., 2002). According to RT QPCR (data not shown) and Western blotting AHSP was not expressed in FL5.12 (Figure 11a). We asked whether 
ectopically expressed AHSP could stabilize endogenous alpha globin and change the viability of FL5.12 cells. In the presence of a FLAG-AHSP IRES GFP expression construct alpha globin protein levels seemed to increase. On Western blot we found that especially the $14.5 \mathrm{kD}$ band of alpha globin increased both in FL5.12 cells growing with or without IL-3. Protein expression of the $14 \mathrm{kD}$ band was not affected by AHSP (Figure 11b). Viability tests performed in the presence or absence of IL-3 demonstrated that DNA fragmentation was comparable in AHSP- and GFP-expressing cells as assessed by TUNEL staining (Table 1).

Our very recent results, however, showed that AHSP was also detected by the hemoglobin antibody: we transiently transfected HeLa cells with a GFP-AHSP or GFP expression vector and prepared separate immunoblots using an anti-AHSP or the anti-hemoglobin antibody. With both antibodies we detected a protein band of $42 \mathrm{kD}$ in GFP-AHSP but not in GFP expressing HeLa cells (Figure 12b). Thus it was not possible to conclude whether the increase of the $14.5 \mathrm{kD}$ band in FL5.12 cells was entirely due to overexpressed FLAG-AHSP or at least in parts due to alpha globin stabilized by AHSP.

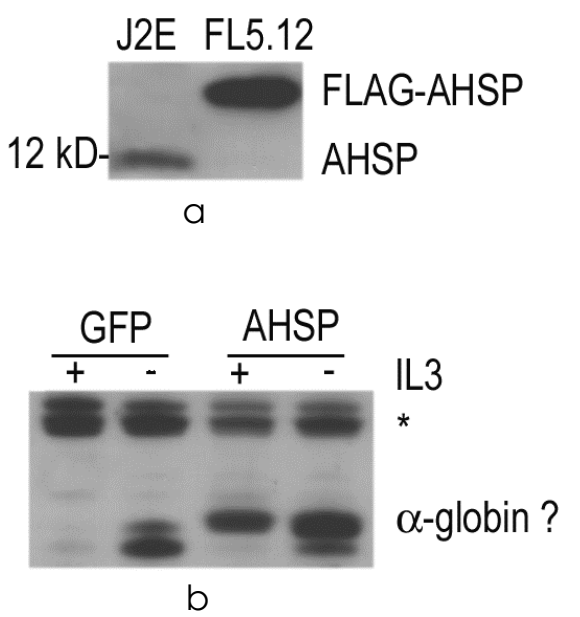

Figure 11: Endogenous AHSP is not expressed in FL5.12 cells and the $14.5 \mathrm{kD}$ alpha globin band increases in FLAG-AHSP expressing cells. Whole cell lysates were prepared from J2E and FL5.12 cells (a) or from GFP or FLAG-AHSP transduced FL5.12 cells growing with or without IL-3 for $12 \mathrm{~h}$ (b). Proteins were analyzed by SDS PAGE and Western blotting. Blots were probed with anti-AHSP (a) or antihemoglobin antibody (b). A background band is marked with an asterisk. 
Table 1: Ectopically expressed FLAG-AHSP does not change the viability of FL5.12 cells.

\begin{tabular}{lcc}
\hline & \multicolumn{2}{c}{ Tunel-positive cells $(\% \pm$ SD) } \\
& $+\mathrm{IL} 3$ & $-\mathrm{IL} 3$ \\
\hline GFP & $0.91 \pm 0.17$ & $30.08 \pm 0.76$ \\
FLAG-AHSP & $0.77 \pm 0.11$ & $26.40 \pm 0.54$ \\
$\begin{array}{l}\text { GFP- } \alpha- \\
\text { globin }\end{array}$ & $1.21 \pm 0.06$ & $49.62 \pm 0.94$ \\
\hline
\end{tabular}

Cells were grown in the presence or absence of IL-3 for $12 \mathrm{~h}$ and were subsequently subjected to TUNEL staining. TUNEL-positive cells were monitored using a FACS Calibur. Each value represents an average of three independent measurements.
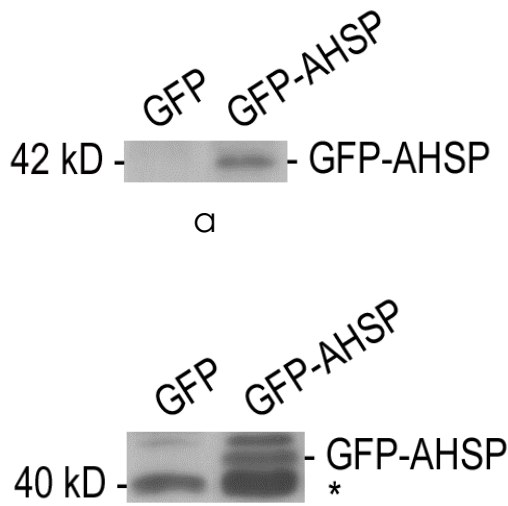

b

Figure 12: The anti-hemoglobin antibody detects AHSP. HeLa cells were transiently transfected with GFP or GFP-AHSP. Whole cell lysates were prepared and proteins were analyzed by SDS PAGE and Western blotting. Blots were probed with anti-AHSP (a) or antihemoglobin antibody (b). A background band is marked with an asterisk.

\subsubsection{NIH3T3, HeLa, and K562 cells differentially express alpha globin upon induction of apoptosis}

To study whether upregulation of alpha globin was unique to FL5.12 cells, we investigated other cell lines of different origin, differentiation status and species. The cells were treated with different cytotoxic compounds. The treatment was stopped and RNA was isolated when $50 \%$ of the cells were dead according to trypan blue staining or microscopic observation. NIH3T3, an immortalized mouse fibroblast cell line, displayed similar regulation of alpha globin mRNA as FL5.12 cells except that the overall expression level of alpha globin was low. While in this cell line alpha globin levels remained low after treatment with STS, cisplatin dramatically induced alpha globin expression both at transcript (60-fold) and at protein level (Figure 13, upper and lower panel). 
The human epithelial cervix cancer cell line, HeLa, displayed opposite expression profiles. While alpha globin was unaltered after treatment with cisplatin, it increased after STS treatment by more than 12-fold (Figure 14, upper panel). Cisplatin or STS did not induce apoptosis in K562 cells (Mc Gee et al., 2001; Dedoussis et al., 1999). However, combined treatment with TRAIL and STI571, an inhibitor of $b c r-a b l$ tyrosine kinase activity (Nimmanapalli et al., 2001), led to apoptosis with elevated alpha globin levels (Figure 14, lower panel). In summary, alpha globin expression increased in all four different cell lines after specific drug treatments.

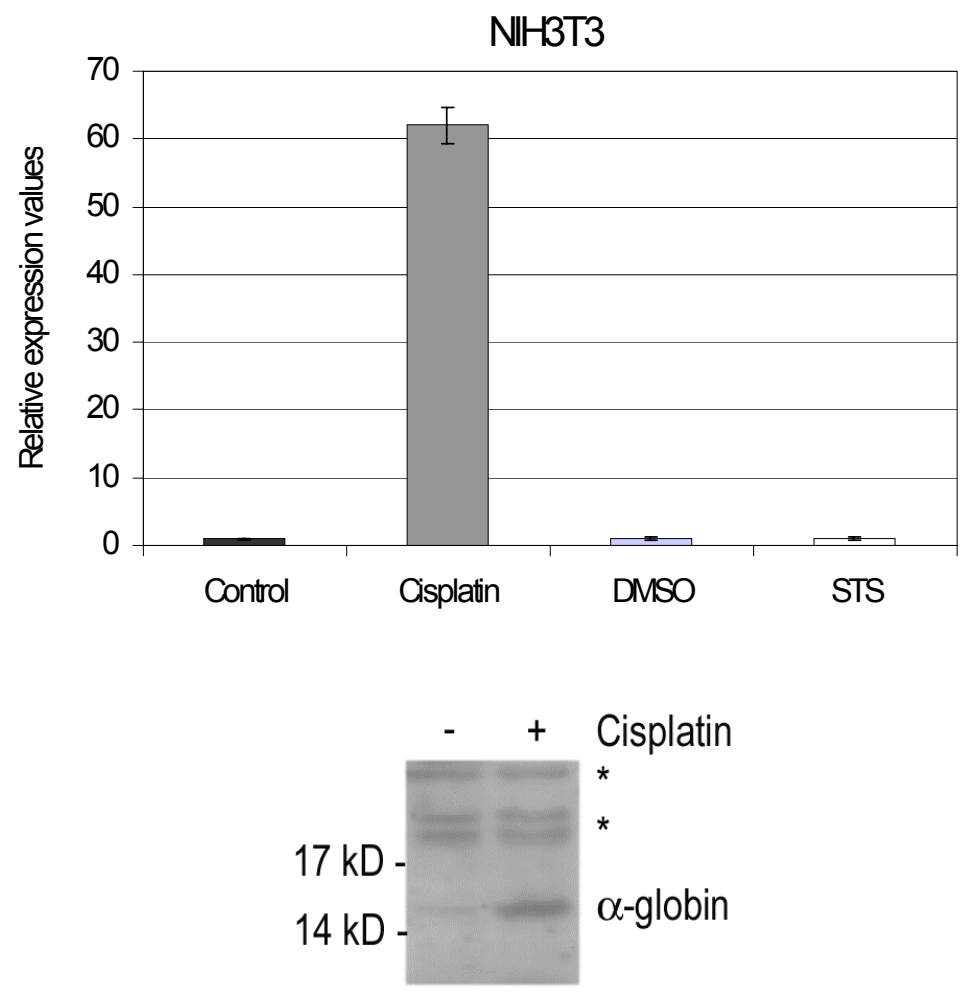

Figure 13: Cisplatin induces expression of alpha globin mRNA and protein in NIH3T3 cells. NIH3T3 cells were treated with $20 \mu \mathrm{M}$ cisplatin or left untreated (Control) for $30 \mathrm{~h}$, or with $1 \mu \mathrm{M}$ STS or DMSO for $24 \mathrm{~h}$. Total RNA (upper panel) and whole cell lysates (lower panel) were isolated. Alpha globin transcript levels were measured by RT QPCR. Each graph represents triplicate measurements of three independent experiments. Proteins were analyzed by SDS PAGE and Western blotting. The blot was probed with anti-hemoglobin antibody. Background bands are marked with asterisks and served to prove equal loading.

We then examined the cell lines for beta globin expression by RT QPCR. NIH3T3 and HeLa cells did not express beta globin under normal or apoptotic conditions. As already mentioned (4.1.2) beta globin is not expressed in K562 cells, so we measured very low levels of beta globin transcript with a detection threshold of 30 cycles. As for FL5.12 cells growing in the absence of IL-3, treatment of K562 cells with STI571/TRAIL, resulted in upregulated beta globin levels by a factor of 4 (Figure 14). 

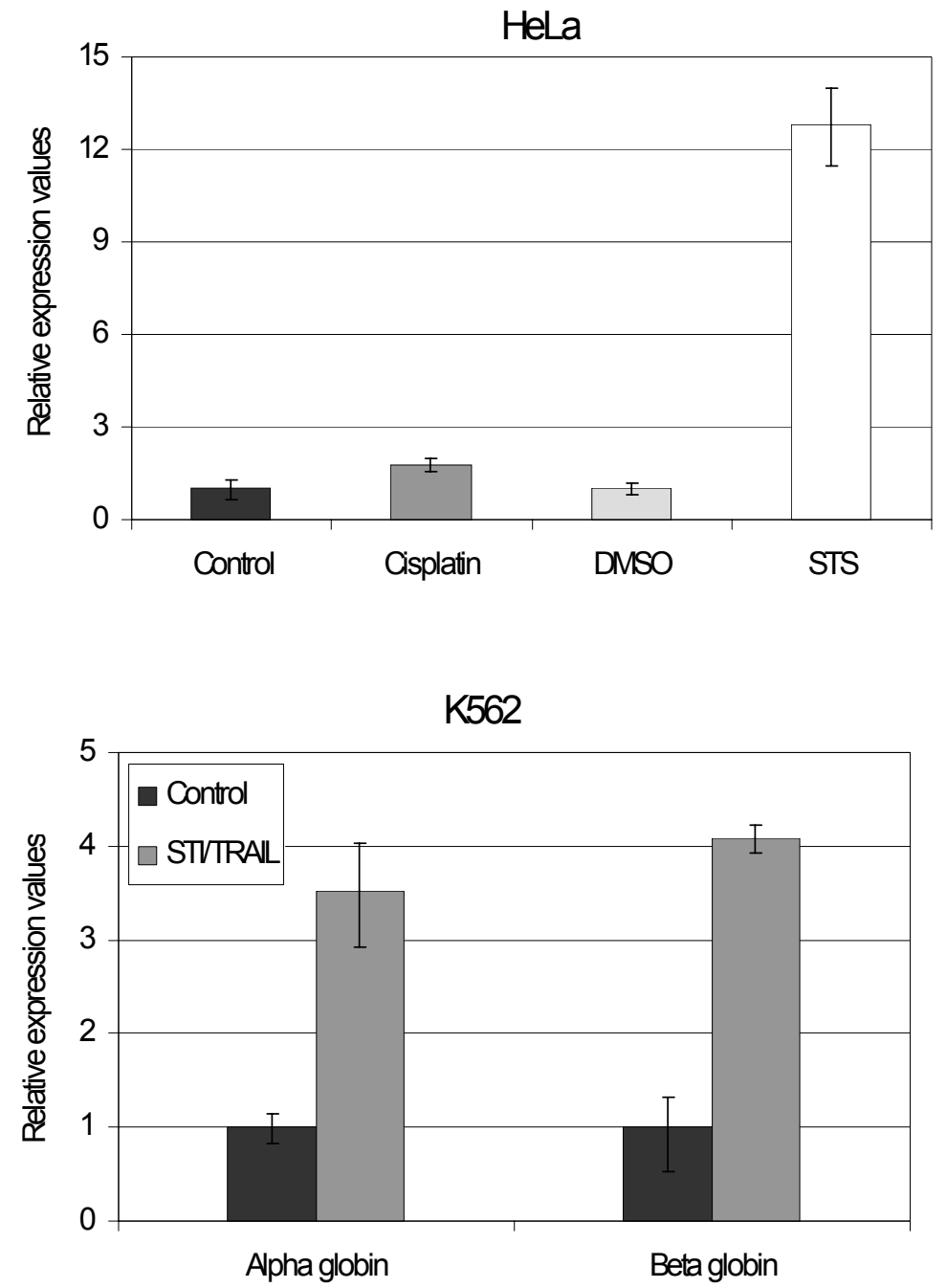

Figure 14: Expression of alpha and beta globin mRNA in HeLa and K562 cells. HeLa cells were treated with $0.5 \mu \mathrm{M}$ STS $(6 \mathrm{~h})$, DMSO $(6 \mathrm{~h}), 20 \mu \mathrm{M}$ cisplatin $(24 \mathrm{~h})$ or left untreated $(24 \mathrm{~h})$ (upper panel). K562 cells were simultaneously treated with $250 \mathrm{ng} / \mathrm{ml}$ TRAIL plus $500 \mathrm{nM}$ STI571 for $48 \mathrm{~h}$ (lower panel). Total RNAs were isolated and alpha and beta globin transcript levels were measured by RT QPCR. Each graph represents triplicate measurements of three independent experiments.

\subsubsection{GFP-alpha globin expressing cells are difficult to enrich}

We investigated the effect of ectopically overexpressed alpha globin in FL5.12 cells. We combined retroviral transduction and subsequent fluorescent activated cell sorting (FACS) to enrich FL5.12 cells expressing alpha globin. In earlier experiments a construct expressing alpha globin with an N-terminal FLAG-tag and an internal ribosomal entry site for GFP (IRES GFP) was used (Brachat et al., 2002). Using this construct high level enrichment of GFP-positive cells was achieved. We tested the presence of alpha globin at both genomic and RNA level using intron 2 spanning, alpha globin-specific primers. PCR on whole cell lysates demonstrated that ectopic alpha globin was inserted into the genome of FL5.12 cells; in both 
untransduced and FLAG-alpha globin transduced cells we detected a $402 \mathrm{bp}$ fragment deriving from intron-containing, genomic alpha globin, whereas only the transduced cells exhibited a strong signal for intron-free alpha globin coding sequence (Figure 15, right panel). RT PCR of FL5.12 cells expressing FLAG-alpha globin showed a stronger band for alpha globin than control cells suggesting that the ectopic alpha globin was transcribed (Figure 15, left panel). However, FLAG-alpha globin was never detected on Western blot suggesting that the cells silenced exogenous FLAG-alpha globin. In our current experiments we used an N-terminal GFP-alpha globin fusion construct to overexpress alpha globin. In contrast to the IRES construct, the fusion construct ensured concomitant sorting of GFP and alpha globin. Transduction efficiencies were evaluated by means of GFP expression $96 \mathrm{~h}$ post transduction using the FACS Calibur. Transduction efficiencies of GFP-alpha globin were low. We measured 6\% GFP-positive cells compared to $82 \%$ and $65 \%$ for GFP-Bax and GFP control clones, respectively (Figure 16). Two rounds of sorting were needed to enrich GFP-alpha globin expressing FL5.12 cells, whereas high expression levels of GFP-Bax were obtained without enrichment. To test the proliferation behavior of GFP, GFP-Bax, and GFP-alpha globin expressing cells as well as untransduced cells in the presence of IL-3, we investigated cell growth in the logarithmic phase over $36 \mathrm{~h}$. Untransduced, GFP and GFP-alpha globin expressing FL5.12 cells displayed very similar proliferation rates. Cells expressing GFP-Bax proliferated slower during the first $14 \mathrm{~h}$. After 20 and $36 \mathrm{~h}$, however, cells adapted growth behavior comparable to GFP-alpha globin expressing and control cells (Figure 17).

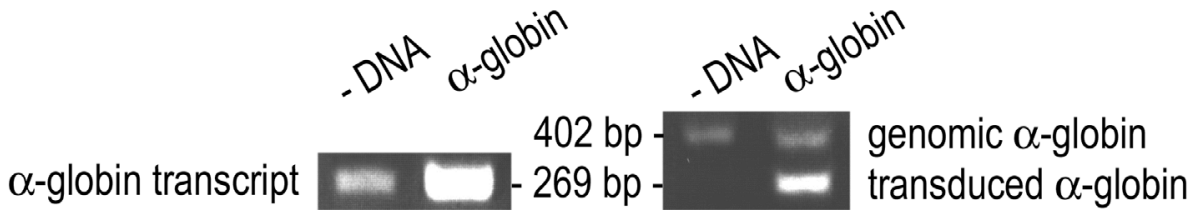

Figure 15: FLAG-alpha globin is inserted into the genome of FL5.12 cells and is transcribed. Genomic DNA and total RNA was isolated from untransduced FL5.12 cells and cells transduced with FLAGalpha globin. Total RNA was reverse transcribed to generate cDNA. cDNA (left) and genomic DNA (right) were subjected to PCR using intron spanning alpha globin specific primers. PCR products were analyzed on a $1 \%$ agarose gel. 

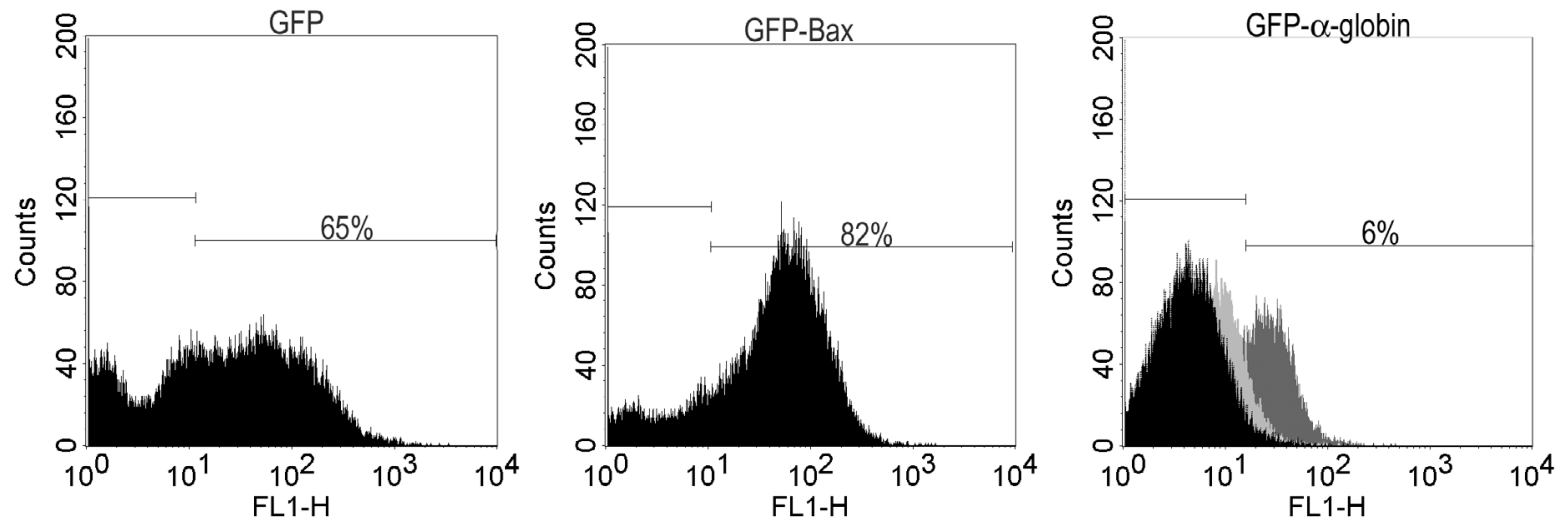

Figure 16: GFP-alpha globin is difficult to express in FL5.12 cells. GFP expression profiles of FL5.12 cells transduced with GFP and GFP-Bax prior to sorting, and GFP-alpha globin prior to sorting (black, 6\%), after one (light grey, 10\%) and two (dark grey, 72\%) rounds of sorting. GFP expression was monitored in the FL1-H channel of a FACS Calibur. The markers depict GFP-positive cells in percentage (\%).

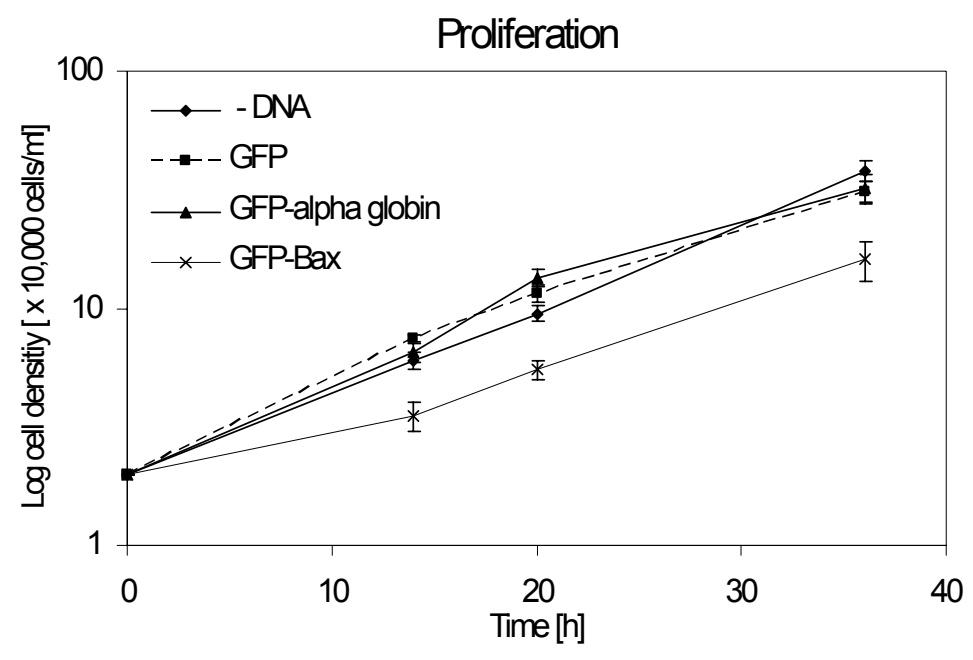

Figure 17: FL5.12 cells expressing GFP-alpha globin display similar proliferation rate as control FL5.12 cells. Untransduced FL5.12 cells or cells expressing GFP, GFP-alpha globin or GFP-Bax were seeded at a density of $2 \times 10^{4}$ cells $/ \mathrm{ml}$. To assess cell viability and density, cells were stained with trypan blue and counted after 14, 20, and $36 \mathrm{~h}$. Each data point represents triplicate measurements.

\subsubsection{GFP-alpha globin accumulates in the cytoplasm and tends to aggregate in FL5.12 cells}

Next, we investigated the localization of GFP-Bcl- $x_{L}$, GFP-Bax, GFP, and GFP-alpha globin in FL5.12 cells by fluorescence microscopy. FL5.12 cells expressing GFP and GFP-Bax showed a homogenous distribution of green fluorescence (Figure 18b, c). In contrast, spontaneously dying GFP-Bax expressing cells, as assessed by DAPI staining, displayed a 
dotted pattern of GFP, suggesting that GFP-Bax had translocated to the mitochondria (Figure 18b) (Hsu et al., 1997). GFP-Bcl- $x_{L}$ was not evenly distributed in the cells. There were areas that displayed higher density of GFP (Figure 18a). Bcl- $\mathrm{x}_{\mathrm{L}}$ has been reported to localize partly to mitochondria, partly to ER but also to the cytosol under normal growth conditions (Gonzalez-Garcia et al., 1994). Further, overexpression of Bcl- $\mathrm{x}_{\mathrm{L}}$ often causes clustering of mitochondria. Thus we assumed that GFP-Bcl- $\mathrm{x}_{\mathrm{L}}$ was associated with membrane compartments in FL5.12 cells.

GFP-alpha globin was mostly evenly distributed in the cytoplasm. In some cells, however, we observed aggregates of GFP. Immunostaining using the hemoglobin-specific antibody efficiently stained the aggregates (Figure 18d). Since a strong signal for GFP alone did not generate a signal in the red channel (Figure 18c) we concluded that the aggregates derived from precipitated overexpressed alpha globin. It has been reported that excess of alpha globin precipitates in erythrocytes and COS cells that do not express the chaperone AHSP, and these aggregates are toxic in erythropoietic cells (Kihm et al., 2002). As described above AHSP is not expressed in FL5.12 cells which may explain the difficulties in enriching GFP-alpha globin in FL5.12 cells. 

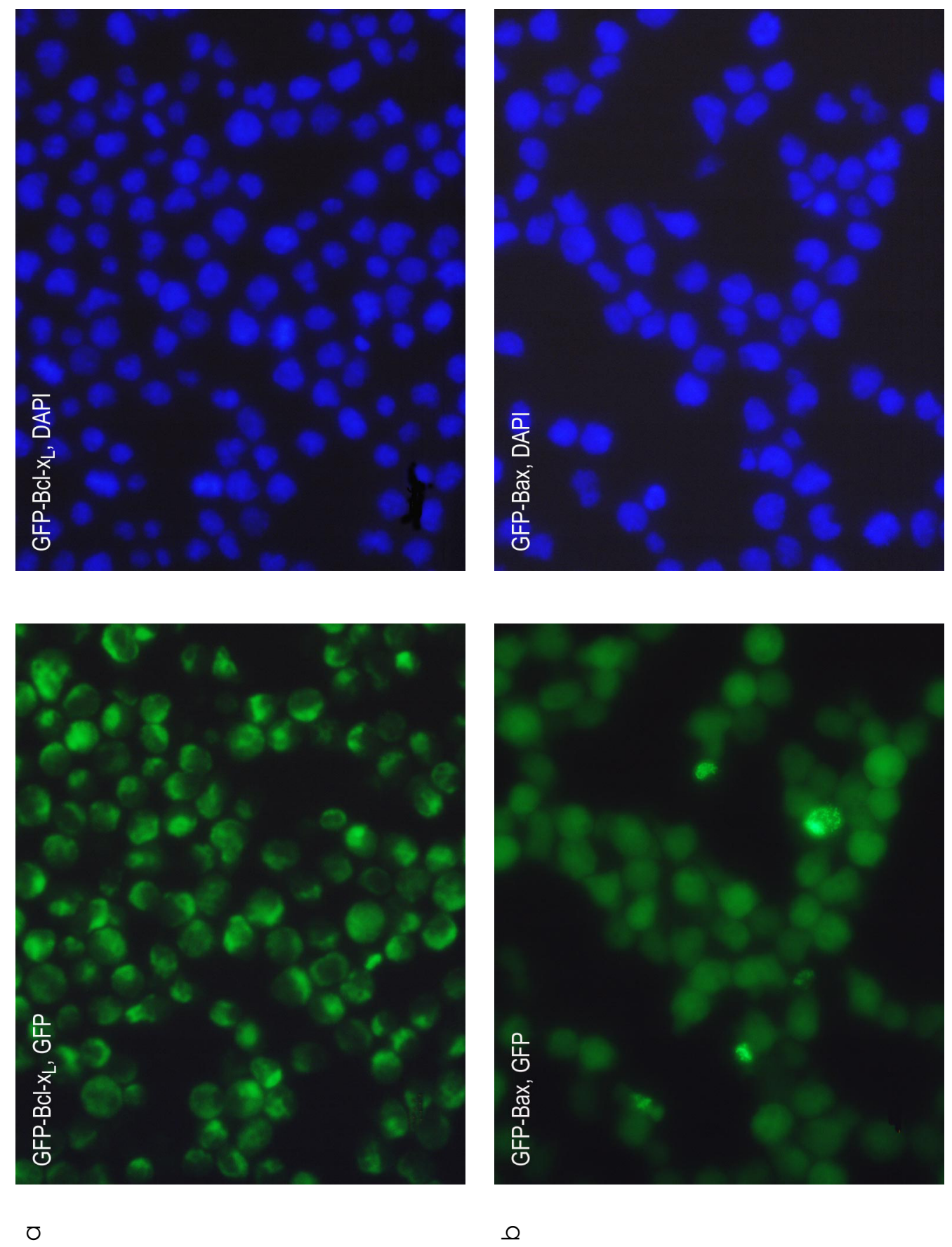


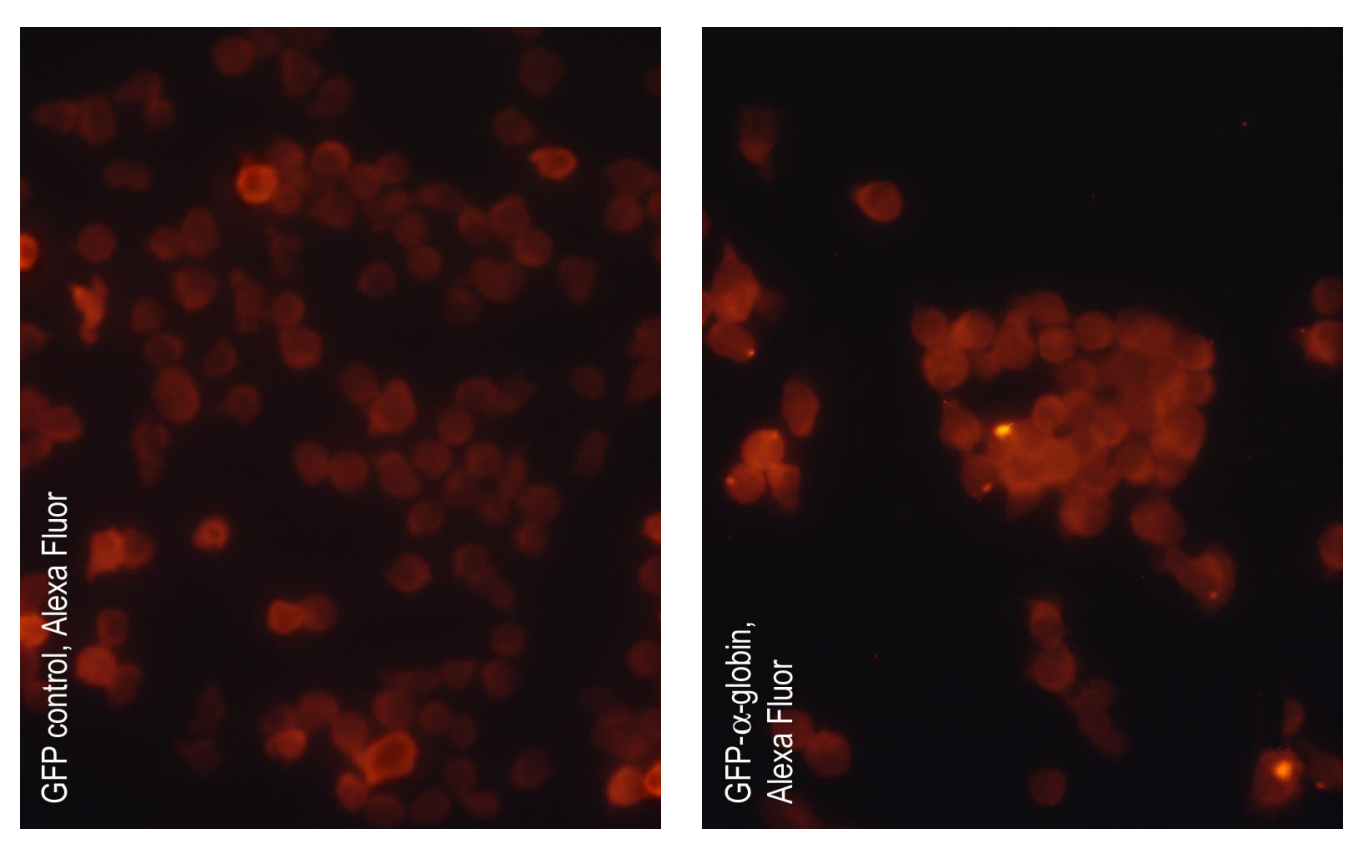

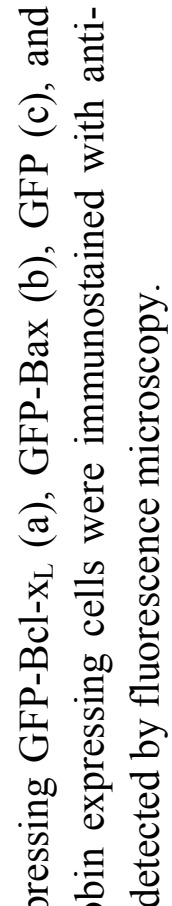
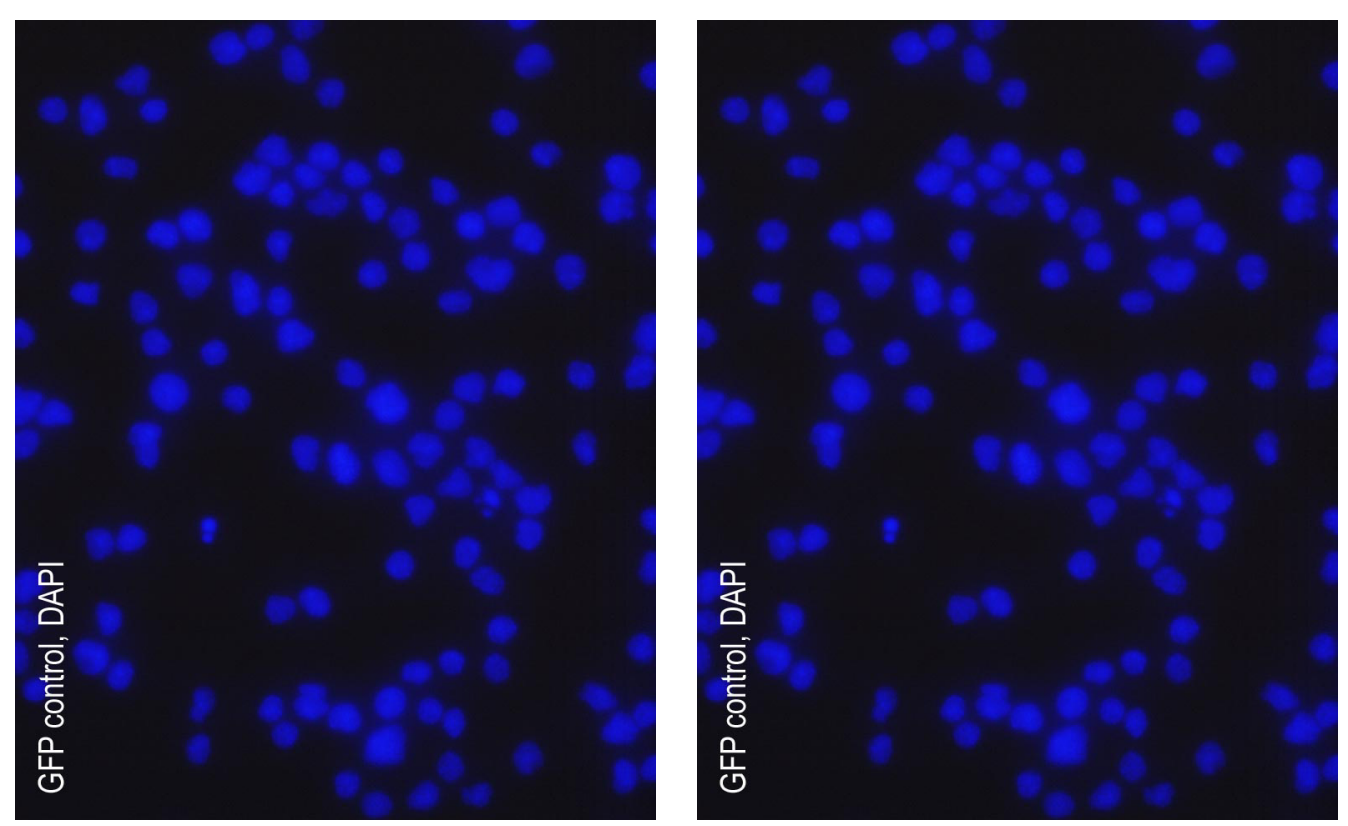

중 이인

$\lambda \vec{\lambda}$

跑 $\frac{2}{0}$

n 0

D

그른

다

记

¿ 0

范市市

응 㟔

不至

o 0

$\approx \beta \dot{\sigma}$

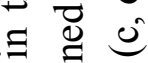

ఖ

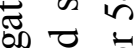

ए ज

का
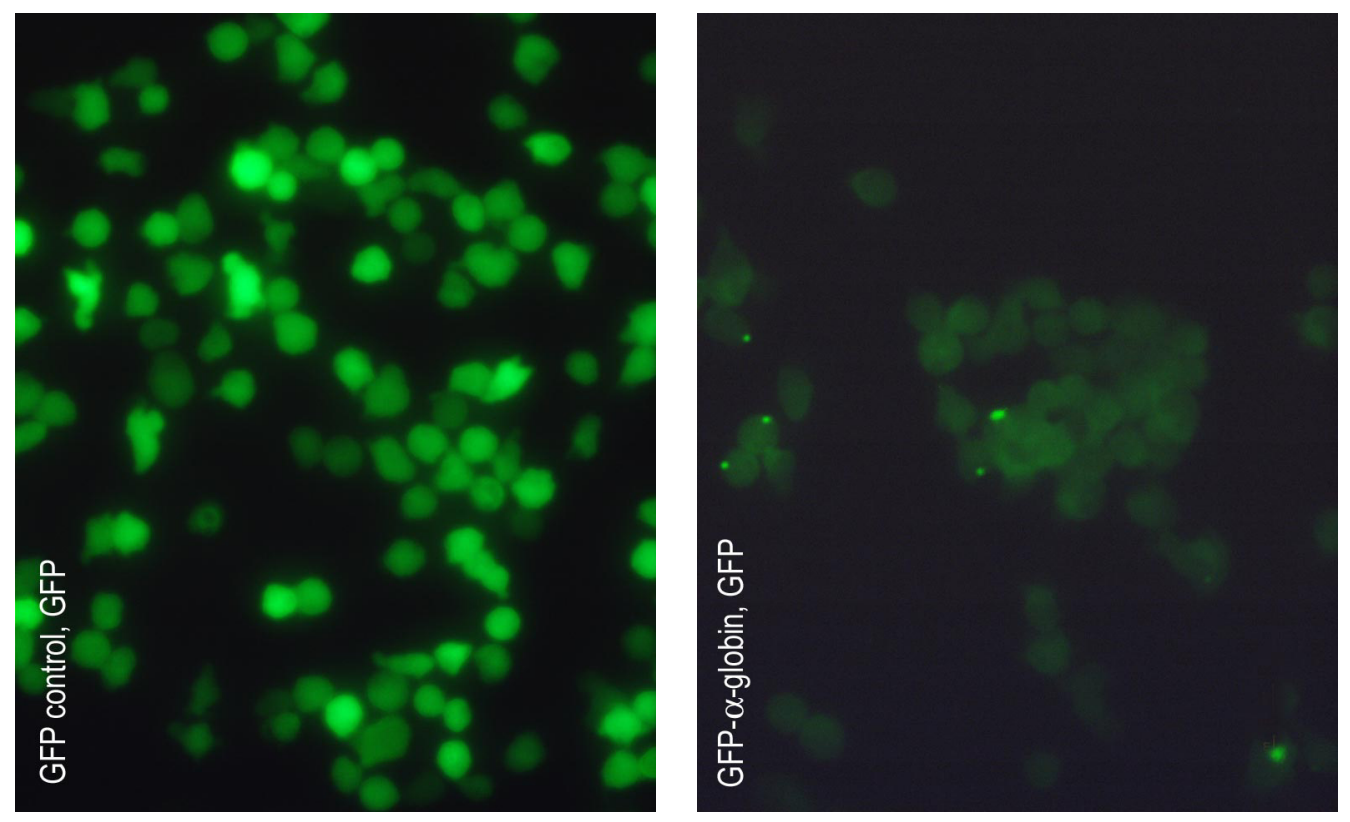

在 结范 ¿ ஓ 飞 궁

in

至家

$\Xi$ त्त्र

$\exists$ 过 융

का 3

귱 항

충

空

0 bo

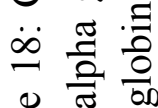

常至 


\subsubsection{GFP-alpha globin is not associated with heme}

In chapter 4.1.6 we have shown that endogenously upregulated alpha globin was not associated with heme. To test whether overexpressed alpha globin was in complex with the prosthetic group we measured heme contents in the twice sorted, GFP-alpha globin overexpressing FL5.12 cells. FL5.12 cells enriched for alpha globin by approximately a factor of 50 (data not shown) exhibited heme levels comparable to those in untransduced or GFP expressing control cells (Figure 19).

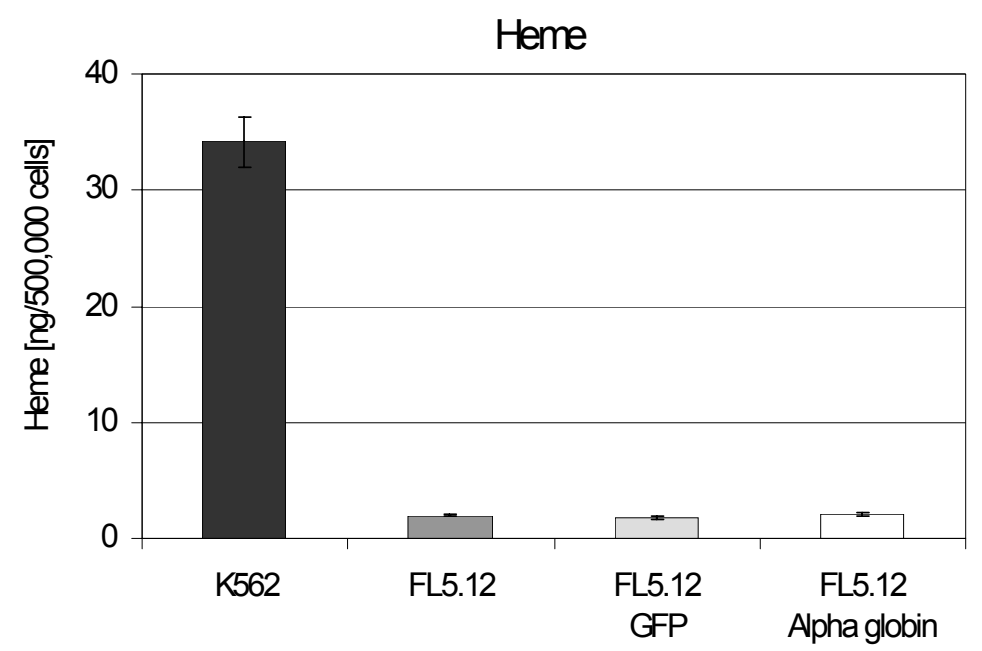

Figure 19: GFP-alpha globin is not associated with heme. Heme contents were measured in FL5.12 cells transduced with GFP or GFPalpha globin (Alpha globin) or untransduced FL5.12 cells and K562 cells as control. The graph represents triplicate measurements of three independent experiments.

\subsubsection{GFP-alpha globin accelerates apoptosis induced by IL-3 deprivation in a dose-dependent manner}

Next we followed apoptosis progression by TUNEL staining in transduced FL5.12 cells after cytokine deprivation. We compared cells expressing GFP-alpha globin which were enriched by one or two rounds of sorting, with cells expressing GFP, GFP-Bcl- $x_{L}$ and GFP-Bax. Protein expression was studied by Western blot using an anti-GFP antibody (Figure 20). Cells were grown in the presence or absence of IL-3 for $12 \mathrm{~h}$, subjected to TUNEL staining and finally analyzed using a FACS machine. All transduced FL5.12 clones growing with IL-3 were TUNEL negative (Figure 21a). Significant differences in the progression of apoptosis were observed after cytokine deprivation. GFP-Bax displayed obvious death-accelerating features; after 12 h 39\% of the cells were TUNEL-positive (Figure 21b), while GFP-Bcl- $x_{L}$ very efficiently rescued cells from death (1\% TUNEL-positive cells) (Figure 21b). GFP-alpha globin expressing cells which were sorted once (1x sorted), died slightly faster than GFP expressing cells (20\% versus $13 \%$ dead cells) (Figure $21 \mathrm{~b}$ ). In contrast, FL5.12 cells expressing higher levels of GFP-alpha globin (2x sorted) displayed 31\% TUNEL-positive cells (Figure 21b). 
We asked whether the pro-apoptotic effect of GFP-alpha globin could be diminished by Bcl$\mathrm{x}_{\mathrm{L}}$. After two rounds of GFP-alpha globin enrichment, we transduced FL5.12 cells with GFPBcl- $\mathrm{x}_{\mathrm{L}}$. Three percent of the cells stained TUNEL-positive after $12 \mathrm{~h}$ without IL-3. This indicates that GFP-Bcl- $x_{L}$ neutralized the pro-apoptotic effect of GFP-alpha globin completely. In summary, GFP-alpha globin accelerated cell death in a dose-dependent manner and this process was neutralized by coexpression of Bcl- $\mathrm{x}_{\mathrm{L}}$. The potencies of GFP-alpha globin and GFP-Bax to induce apoptosis in cytokine-deprived FL5.12 cells were comparable.

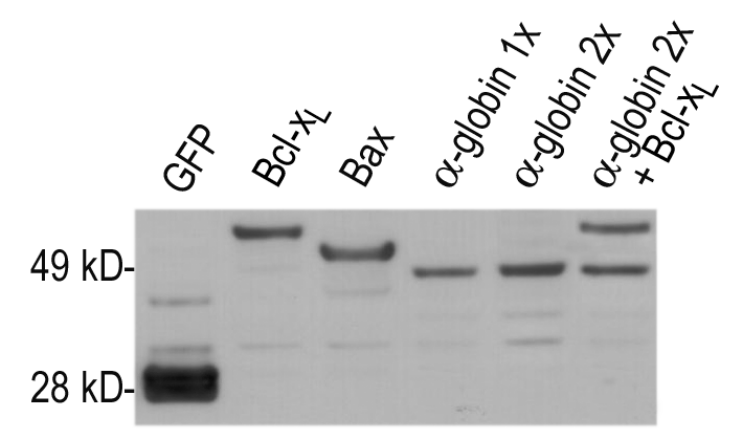

Figure 20: Control of expression of GFP fusion proteins in FL5.12 cells. Whole cell lysates of FL5.12 cells transduced with GFP, GFPBcl- $\mathrm{x}_{\mathrm{L}}\left(\mathrm{Bcl}-\mathrm{x}_{\mathrm{L}}\right)$, GFP-Bax (Bax), once and twice sorted GFP-alpha globin ( $\alpha$-globin $1 \mathrm{x}$ and $\alpha$-globin $2 \mathrm{x}$ ), and GFP-alpha globin plus GFP-Bcl- $x_{\mathrm{L}}\left(\alpha\right.$-globin $\left.2 \mathrm{x}+\mathrm{Bcl}-\mathrm{x}_{\mathrm{L}}\right)$. Proteins were analyzed by SDS PAGE and Western blotting. The blot was probed with anti-GFP antibody. 


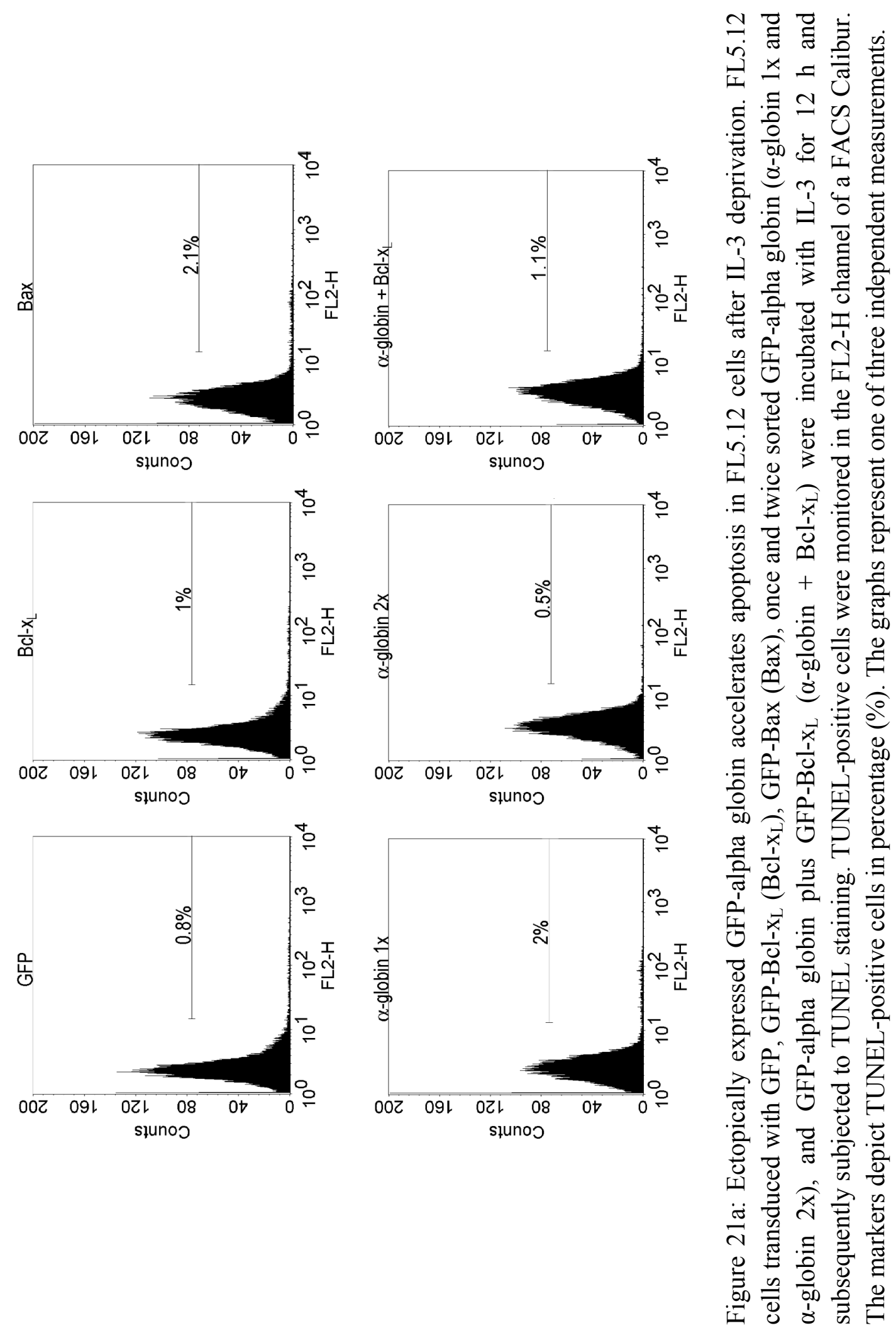




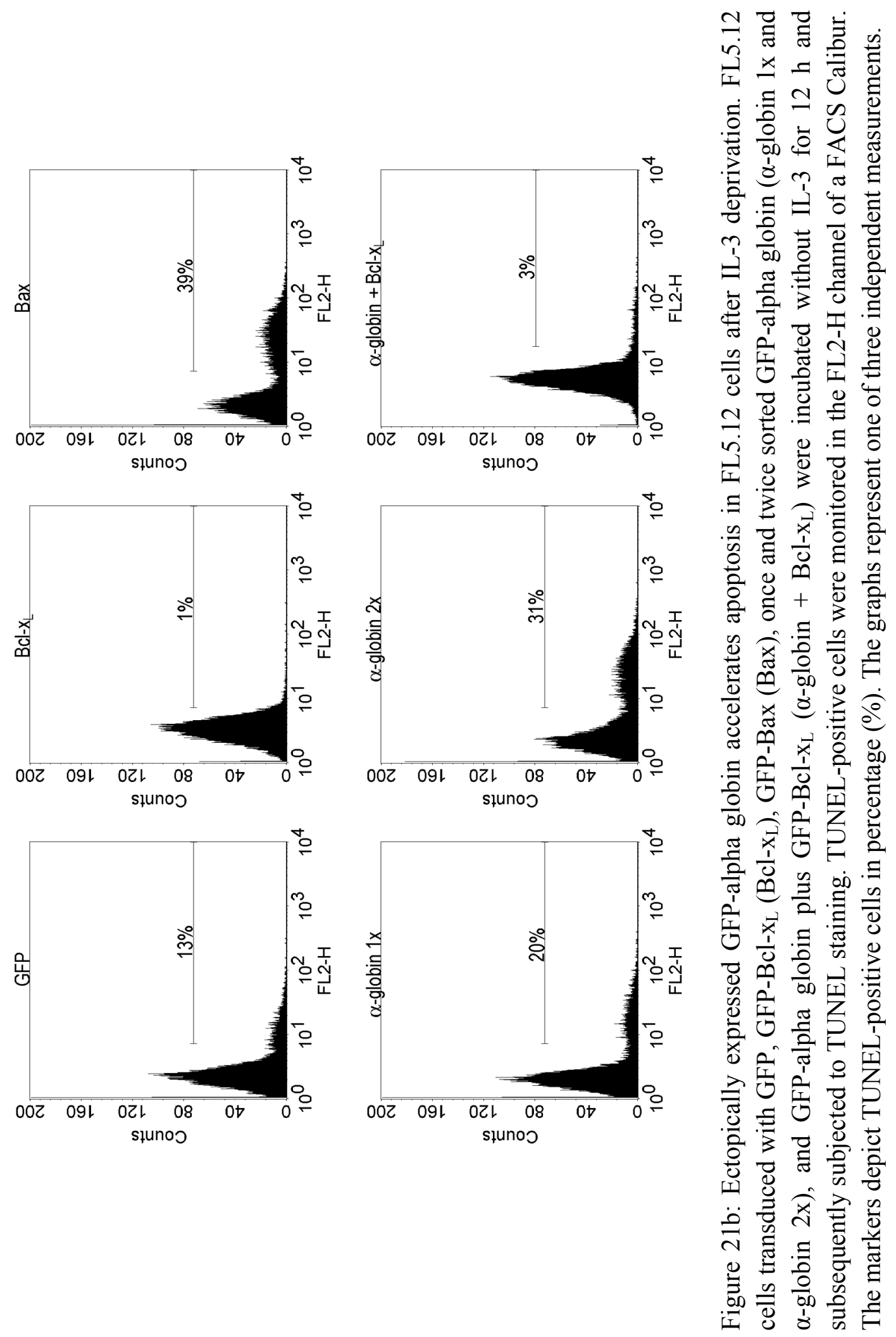




\subsubsection{GFP-alpha globin accelerates cell death in cisplatin-treated NIH3T3 cells}

Initial experiments with GFP-, GFP-alpha globin-, and GFP-Bax transduced NIH3T3 cells corroborated the observations made for FL5.12 cells. First, NIH3T3 cells tolerated accumulation of both alpha globin and Bax under normal growth conditions. Second, apoptosis induction with cisplatin caused faster cell death in NIH3T3 cells overexpressing GFP-alpha globin or GFP-Bax than in cells expressing GFP alone, as evaluated by ATP depletion in the CellTiter-Glo Luminescent Cell Viability Assay (Table 2). GFP-alpha globin expressing NIH3T3 cells showed lowest luciferase activity which was $80 \%$ of the activity observed in GFP control cells. In comparison, ATP content in GFP-Bax enriched cells was about $86 \%$ compared to GFP transduced cells. The assay is based on the theory that the mitochondrial membrane potential, which is the driving force for mitochondrial ATP synthesis, declines during apoptosis and results in decreased ATP levels (Richter et al., 1996). The added luciferase reacts with luciferin in the presence of cellular ATP. The generated fluorescent signal represents the number of viable cells in culture.

Table 2: GFP-alpha globin and GFP-Bax accelerate cell death in cisplatin-treated NIH3T3.

\begin{tabular}{|c|c|c|c|c|c|c|}
\hline & \multicolumn{2}{|c|}{ Control } & \multicolumn{2}{|c|}{ Cisplatin } & \multirow[b]{2}{*}{ Ratio } & \multirow[b]{2}{*}{$\begin{array}{l}\text { Relative } \\
\text { activity }\end{array}$} \\
\hline & $\begin{array}{c}\text { Luciferase } \\
\text { signal (cps } x \\
1000)\end{array}$ & SD & $\begin{array}{c}\text { Luciferase } \\
\text { signal (cps x } \\
1000)\end{array}$ & SD & & \\
\hline GFP & 384.5 & 22.5 & 138.2 & 11.2 & 0.36 & 1.00 \\
\hline GFP-Bax & 390.7 & 16.6 & 120.9 & 27.0 & 0.31 & 0.86 \\
\hline GFP- $\alpha$-globin & 398.1 & 20.4 & 115.1 & 8.4 & 0.29 & 0.80 \\
\hline
\end{tabular}

GFP, GFP-alpha globin or GFP-Bax overexpressing NIH3T3 cells growing in a 96-well optical plate were treated with $20 \mu \mathrm{M}$ cisplatin or left untreated for $20 \mathrm{~h}$. Viability was assessed using CellTiter-Glo ${ }^{\mathrm{TM}}$ Luminescent Cell Viability Assay. Luminescence was measured in counts per second (cps x 1000). Luciferase signals represent triplicate measurements of three independent experiments. SD depicts the standard deviations; the Ratio indicates the ratio of the luciferase signal of cisplatin-treated versus control cells; Ratios obtained for GFP transduced cells served as reference and were set as 1.

\subsubsection{GFP-alpha globin reduces upregulation of endogenous alpha globin under cytokine-deprived conditions}

Interestingly, in FL5.12 cells overexpressing GFP-alpha globin we observed diminished upregulation of endogenous alpha globin at protein level (Figure 22). This suggests that the observed enhancement of apoptosis in transduced FL5.12 cells is due to the GFP-alpha globin, and upregulation of endogenous alpha globin might be prevented by negative feedback inhibition. 


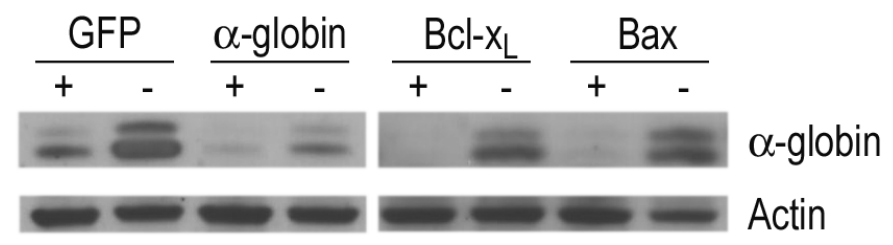

Figure 22: Ectopically expressed GFP-alpha globin reduces upregulation of endogenous alpha globin protein. FL5.12 cells ectopically expressing GFP, GFP-Bcl- $\mathrm{x}_{\mathrm{L}}\left(\mathrm{Bcl}-\mathrm{x}_{\mathrm{L}}\right)$, GFP-Bax (Bax) or twice sorted GFP-alpha globin ( $\alpha$-globin) were grown in the presence or absence of IL-3 for $14 \mathrm{~h}$. Whole cell lysates were analyzed with $12 \%$ SDS PAGE and blotted with anti-hemoglobin antibody.

\subsubsection{Pan caspase inhibitor, zVAD-fmk, delays apoptosis in FL5.12 cells expressing GFP-alpha globin and GFP-Bax}

We next asked whether the activity of caspases was enhanced in apoptosis accelerated by GFP-alpha globin. To assess whether caspases in general were activated in cytokine-deprived FL5.12 cells, we first tested the inhibitory effect of zVAD-fmk, a broad-spectrum caspase inhibitor, compared to DMSO as control in untransduced FL5.12 cells. Cells treated with $50 \mu \mathrm{M} z \mathrm{VAD}$-fmk or DMSO displayed $29 \%$ and 50\% TUNEL-positive cells, respectively, in the absence of IL-3 (Figure 23), i.e. a significant but moderate inhibition of apoptosis by zVAD-fmk was observed after cytokine-withdrawal from FL5.12 cells. Even higher doses of zVAD-fmk (100 $\mu \mathrm{M}$ and $200 \mu \mathrm{M})$ were not found to fully abrogate apoptosis (data not shown). This suggests that either caspase-independent processes were involved or that the concentration of zVAD-fmk was still too low. While pan-caspase inhibitor zVAD-fmk delayed apoptosis in IL3-deprived FL5.12 cells, zVAD-fmk did not prevent upregulation of alpha globin expression either at RNA (Figure 24, upper panel) or at protein level (Figure 24, lower panel).
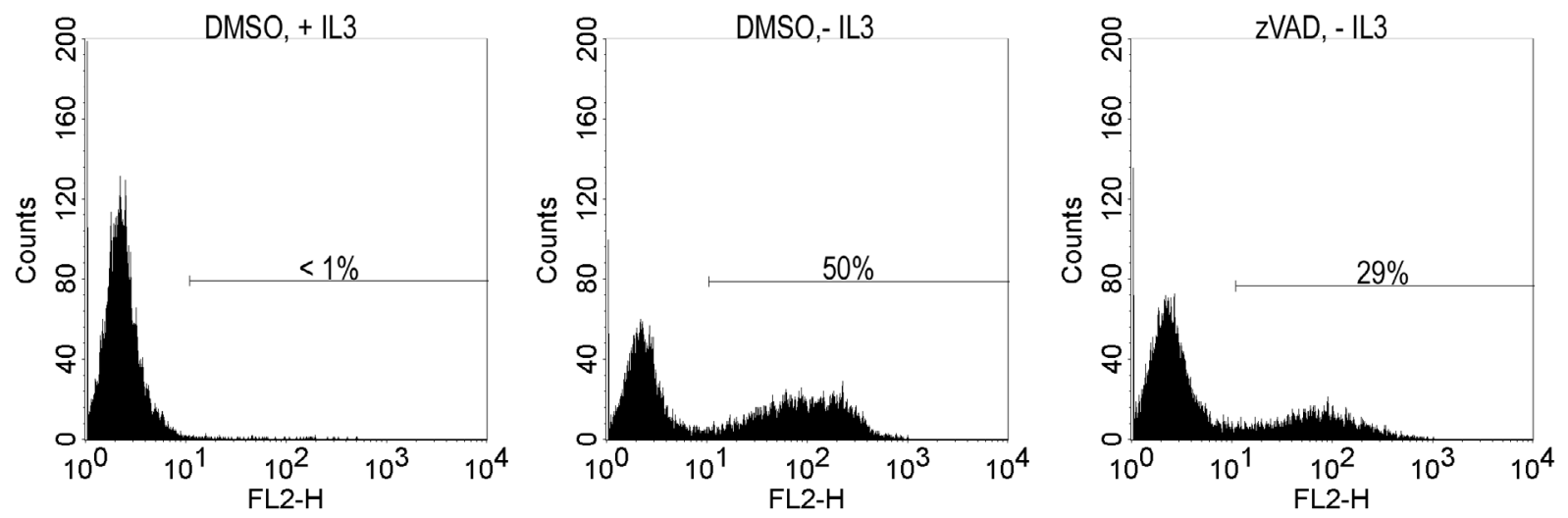

Figure 23: zVAD-fmk diminishes apoptosis after cytokine deprivation in FL5.12 cells. FL5.12 cells were grown in the presence or absence of IL-3 for $12 \mathrm{~h}$. Cells growing without IL-3 were either treated with $50 \mu \mathrm{M}$ zVAD-fmk or DMSO as control. Cells were subsequently subjected to TUNEL staining and TUNELpositive cells were monitored using a FACS Calibur. The markers depict TUNEL- 
positive cells in percentage (\%). The graphs represent triplicate measurements of three independent experiments.

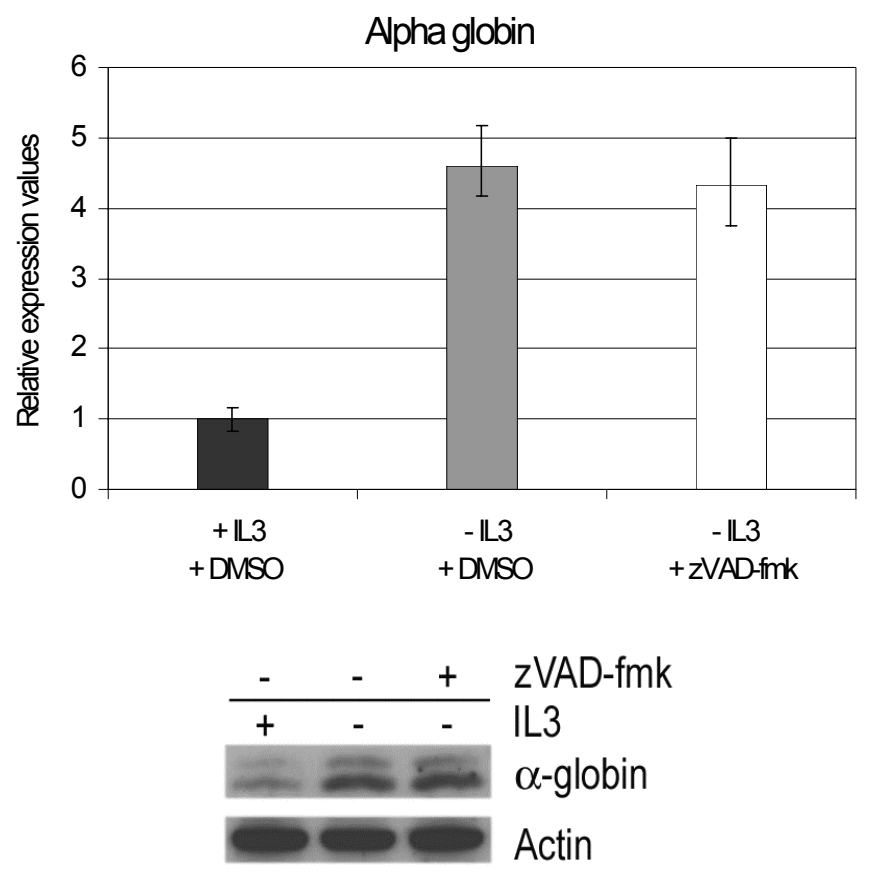

Figure 24: zVAD-fmk does not prevent upregulation of alpha globin. FL5.12 cells were grown in the presence or absence of IL-3 for $12 \mathrm{~h}$. Cells growing without IL-3 were either treated with $50 \mu \mathrm{M}$ zVAD-fmk or DMSO as control. In parallel total RNA and protein were isolated. Alpha globin transcript levels were evaluated using RT QPCR (upper panel). Whole cell lysates were analyzed by SDS PAGE and Western blotting using the rabbit anti-hemoglobin antibody (lower panel).

After we had shown that caspases were generally involved in IL-3 deprivation induced cell death we measured DNA degradation in FL5.12 cells expressing GFP, GFP-Bax, or GFPalpha globin in the presence of zVAD-fmk or DMSO after 0,14 , and $22 \mathrm{~h}$ by TUNEL staining. The effect of zVAD-fmk was most evident in the early phase of apoptosis in cells expressing GFP. Cells expressing GFP-alpha globin and GFP-Bax died significantly faster, 3- and 3.6-fold, respectively, than GFP expressing cells. Moreover, cell death inhibition by $\mathrm{zVAD}$-fmk was less pronounced in these cells than in GFP expressing cells. In the presence of zVAD-fmk, progression of apoptosis after $14 \mathrm{~h}$ was diminished by a factor of 4 in GFP compared to less than factor 2 in GFP-alpha globin and GFP-Bax expressing cells (Figure 25). 


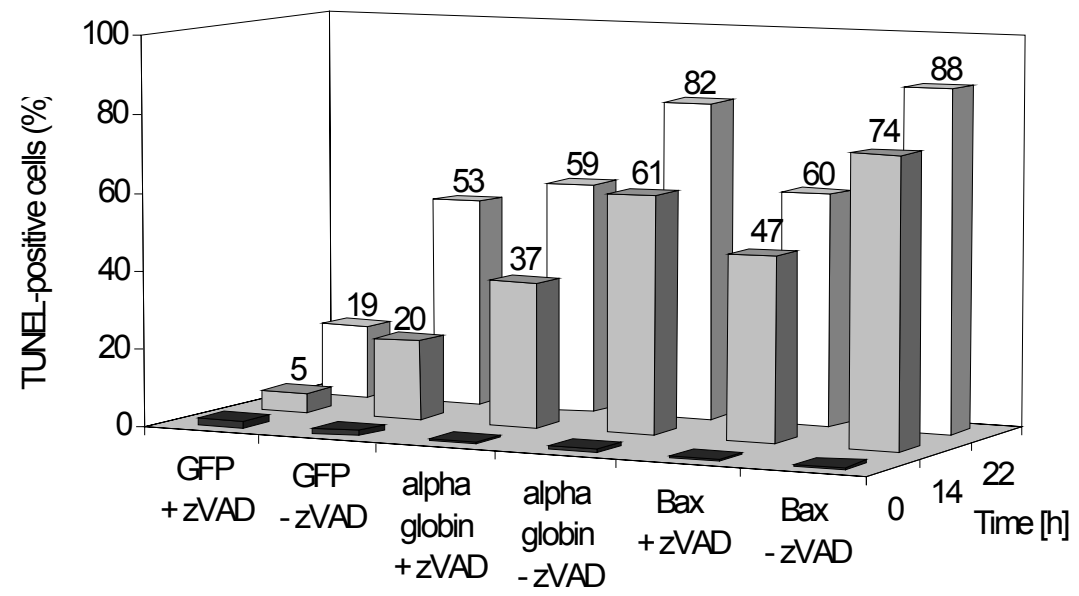

Figure 25: The inhibitory effect of zVAD-fmk is less pronounced in FL5.12 cells overexpressing GFP-Bax or GFP-alpha globin under cytokine-deprived conditions. FL5.12 cells transduced with GFP, GFP-Bax (Bax), or GFP-alpha globin (alpha globin) were incubated without IL-3 either in the presence or absence of $50 \mu \mathrm{M}$ zVAD-fmk (zVAD). After 0,14 , and $22 \mathrm{~h}$ cells were subjected to TUNEL staining and TUNEL-positive cells were analyzed using a FACS Calibur. Each bar represents triplicate measurements.

\subsubsection{Caspase -3 and -9 are highly activated, caspase -8 is moderately activated in GFP-alpha globin overexpressing cells in the absence of IL-3}

To study which caspases are involved in FL5.12 cells in IL-3 deprivation induced apoptosis, we performed caspase-profiling of caspases-2, -3, -8, and -9 using the ApoAlert Caspase Assay Plate. Untransduced FL5.12 cells, cells ectopically expressing GFP, GFP-Bcl- $\mathrm{x}_{\mathrm{L}}$, GFPBax, GFP-alpha globin (once and twice sorted), and cells cotransduced with GFP-alpha globin and GFP-Bcl- $\mathrm{x}_{\mathrm{L}}$ were grown with or without IL-3 for $12 \mathrm{~h}$. Cell lysates were incubated with substrates for the corresponding caspases in the presence or absence of the specific caspase-inhibitor. According to profiling, caspase- 3 and -2 were significantly activated. Caspase- 8 was processed to only some extent, while caspase- 9 was very weakly activated (Figure 26). The caspase inhibitors, especially those of caspase- 8 and caspase- 2 have been shown to prevent caspase activity inefficiently. For all activated caspases tested we found that GFP-Bax and GFP-alpha globin expressing cells displayed higher caspase activities than control cells. Both the dose-dependent effect of sequentially enriched alpha globin and the protecting properties of $\mathrm{Bcl}-\mathrm{x}_{\mathrm{L}}$ in alpha globin expressing cells, as seen in the TUNEL staining, were confirmed. However, the caspase profiling plate provide only crude information about caspase activities; for example the substrate for caspase-2, VDVAD-AMC, can also be efficiently cleaved by recombinant caspase-3 ((Troy and Shelanski, 2003) and our own data, not shown) and caspase-7. Furthermore, caspase-7 and -3 share substrate specificity. 
To ascertain which caspases were involved in apoptosis induced by IL-3 deprivation in FL5.12 cells, we studied caspase expression and activation using Western blot analysis. We used whole cell lysates of FL5.12 cells which were grown either in the presence or absence of IL-3 for $14 \mathrm{~h}$. Executioner caspase-3 was processed and we observed cleaved caspase-3 in apoptotic but not in healthy FL5.12 cells (Figure 27). Caspase-7 was neither detected as full length nor as cleaved protein. The intensity of the $55 \mathrm{kD}$ band representing full length caspase- 2 decreased and an additional protein band of $16 \mathrm{kD}$, probably deriving from cleaved caspase-2, was detected in FL5.12 cells growing in the absence but not in the presence of IL-3 (Figure 27). We therefore suggest slight activation of caspase-2 under cytokine-deprived conditions. As caspase-2 was only weakly activated according to Western blot results, we concluded that activated caspase- 3 accounted at least partially for the strong activation of caspase- 2 substrate in profiling. Further, caspase- 9 was processed in cytokine-deprived but not control FL5.12 cells (Figure 27). This suggests that the cytochrome c/Apaf-1/caspase-9 apoptosome was involved in apoptosis induced by IL-3 withdrawal in FL5.12 cells. Interestingly, cleaved caspase- 8 was also detected on Western blot, thus confirming data from the caspase profiling experiment. We compared caspase- 8 activation in FL5.12 cells after IL-3 deprivation and CHX/TNF alpha treatment and found that processing of caspase- 8 in FL5.12 cells growing without IL-3 was weak compared to cells stimulated by CHX/TNF alpha.

Caspase-2

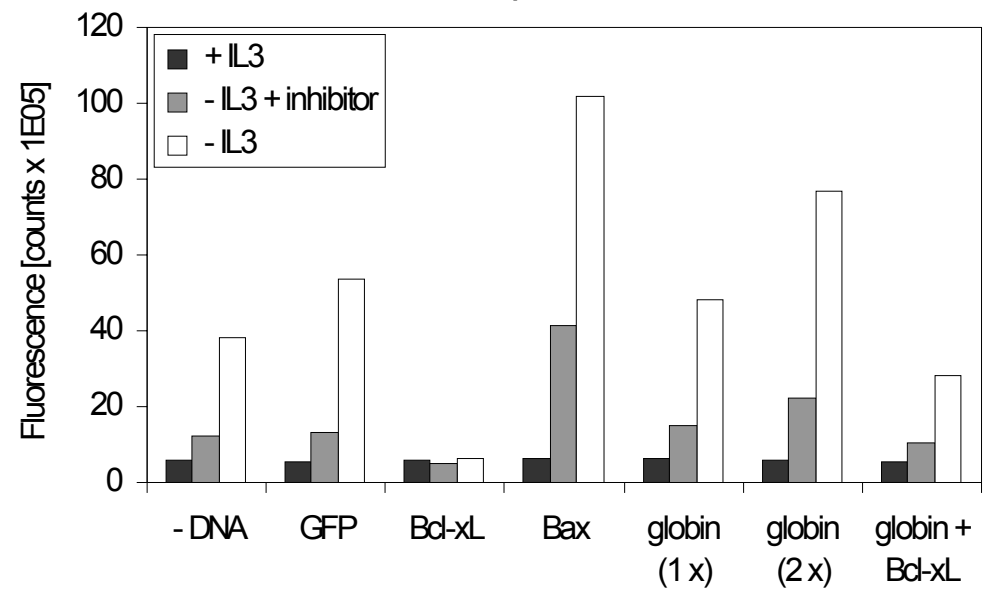

Caspase-3

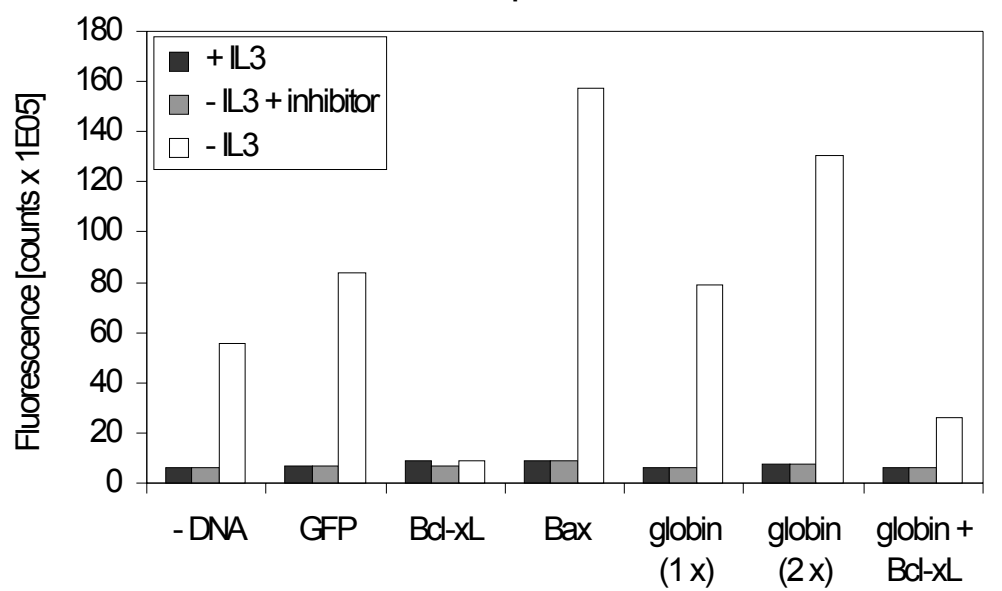



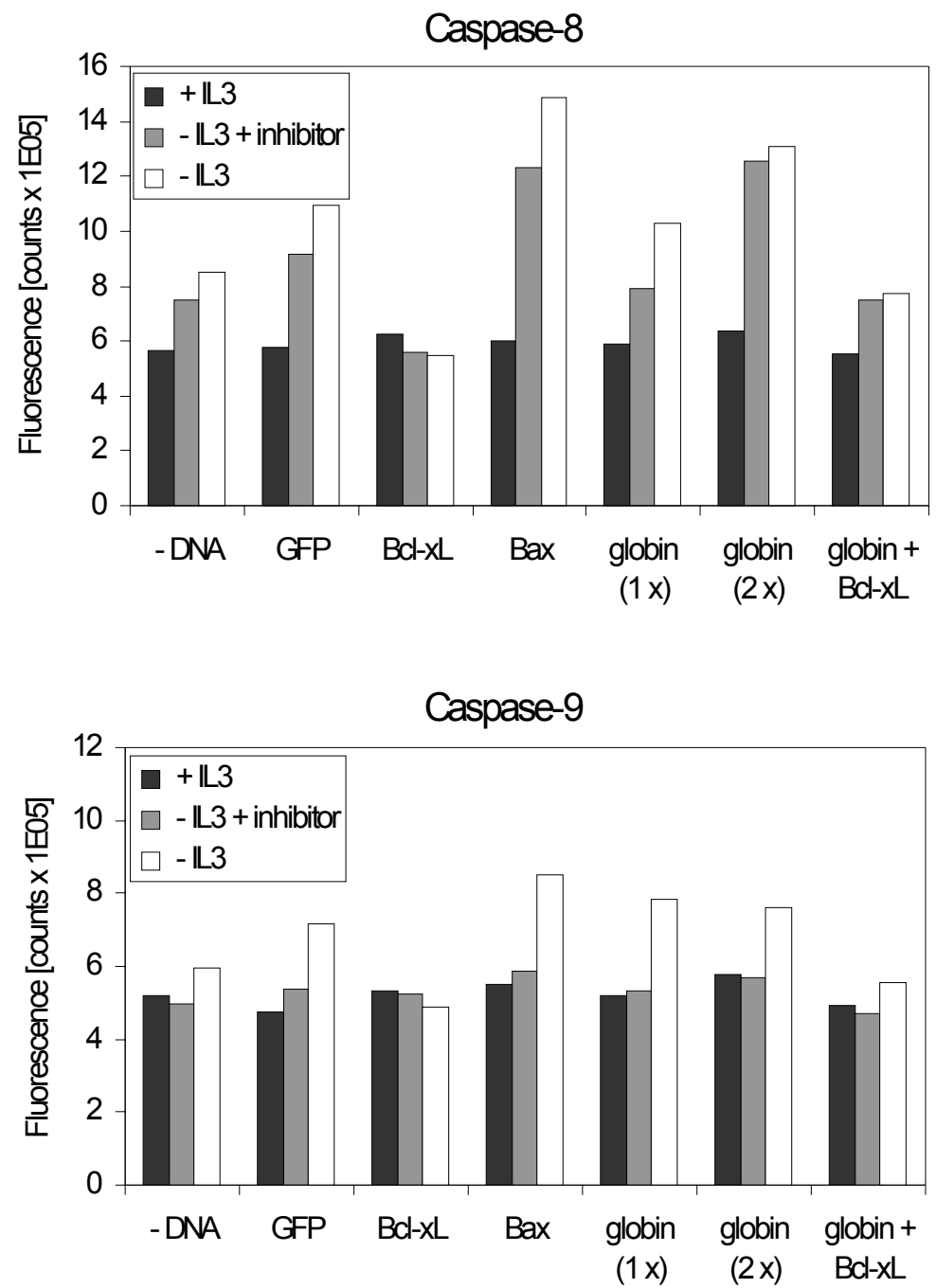

Figure 26: Caspase profiling in cytokine-deprived FL5.12 cells. Untransduced FL5.12 cells, cells ectopically expressing GFP, GFPBcl- $x_{L}\left(B c l-x_{L}\right)$, GFP-Bax $(B a x)$, cells once and twice enriched for GFP-alpha globin (globin 1x, globin $2 \mathrm{x}$ ) or cells cotransduced with GFP-alpha globin and GFP-Bcl- $\mathrm{x}_{\mathrm{L}}\left(\right.$ globin $\left.+\mathrm{Bcl}-\mathrm{x}_{\mathrm{L}}\right)$ were grown with or without IL-3 for $12 \mathrm{~h}$. Cell lysates were tested for the corresponding caspases in the presence or absence of the specific caspase inhibitor. Single measurements were performed for cells growing with IL-3 and cells growing without IL-3 plus the specific caspase inhibitor. Duplicates were analyzed for cells growing in medium lacking IL-3. Fluorescence intensity was monitored after $1.5 \mathrm{~h}$ (405 nm excitation, $460 \mathrm{~nm}$ emission). The caspase profiling represents one of two independently performed experiments. 


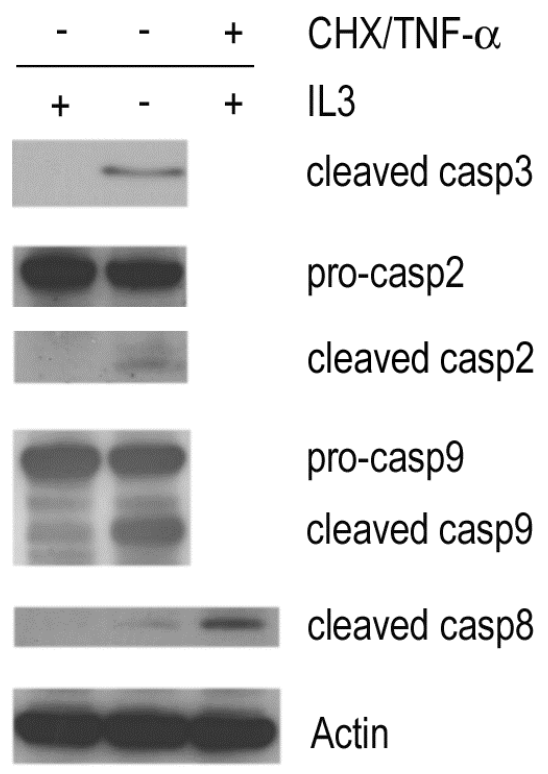

Figure 27: Caspase activation in cytokine-deprived FL5.12 cells. Caspase-2, $-3,-9$, and -8 are cleaved in FL5.12 cells after withdrawal of IL-3. Whole cell lysates of FL5.12 cells growing with or without IL-3 or treated with $1 \mu \mathrm{g} / \mathrm{ml}$ CHX plus $2 \mathrm{ng} / \mathrm{ml} \mathrm{TNF}$ alpha for $14 \mathrm{~h}$, were analyzed with SDS PAGE and Western blotting. Separate blots were prepared and in parallel probed with anti-caspase-2, anti-cleaved caspase-3, anti-caspase- 8 , and anti-caspase- 9 antibody.

\subsubsection{Cytochrome C, Bid, and Bax are processed with faster kinetics in GFP-alpha globin expressing cells}

The observation of activated caspase- $9,-8$ and -2 prompted us to study other candidates that signal through the mitochondrial pathway. We performed a time course experiment with FL5.12 cells expressing GFP, GFP-alpha globin or GFP-Bax in the presence or absence of IL-3. After $0,10,14$, and $22 \mathrm{~h}$, we separated whole cell lysates into cytosolic and membrane protein fractions by ultracentrifugation and investigated protein expression and localization of Bid, Bax, and cytochrome c by Western blotting. In general, after $22 \mathrm{~h}$ without IL-3, apoptosis has progressed so far that almost all proteins were degraded. In Bax and alpha globin expressing cells full length Bid decreased faster than in GFP expressing cells (Figure 28, upper panel). Truncated, pro-apoptotic Bid has been reported to translocate to mitochondria, recruit and oligomerize Bax and permeabilize mitochondria (Luo et al., 1998). Therefore, we investigated Bax localization and found that Bax translocated from the cytosol to the membrane fraction in all three investigated cell lines (Figure 28, upper and lower panel). Again translocation occurred with fastest kinetics in Bax overexpressing cells. Both endogenous and GFP-Bax redistributed from the cytosolic to the membrane fraction suggesting that ectopically expressed Bax promoted the apoptotic process. Accelerated Bax translocation was also detected in alpha globin but not in GFP expressing cells (Figure 28, Table 3). Last, we tested whether cytochrome c was released into the cytosol. In Bax as well 
as in alpha globin expressing cells we observed cytosolic cytochrome c after $10 \mathrm{~h}$, but after $14 \mathrm{~h}$ in GFP control clones.

In summary, under conditions without IL-3 in all cells cleavage of Bid and translocation of Bax and cytochrome c were observed. These phenomena occurred with faster kinetics in both GFP-alpha globin and GFP-Bax expressing FL5.12 cells.

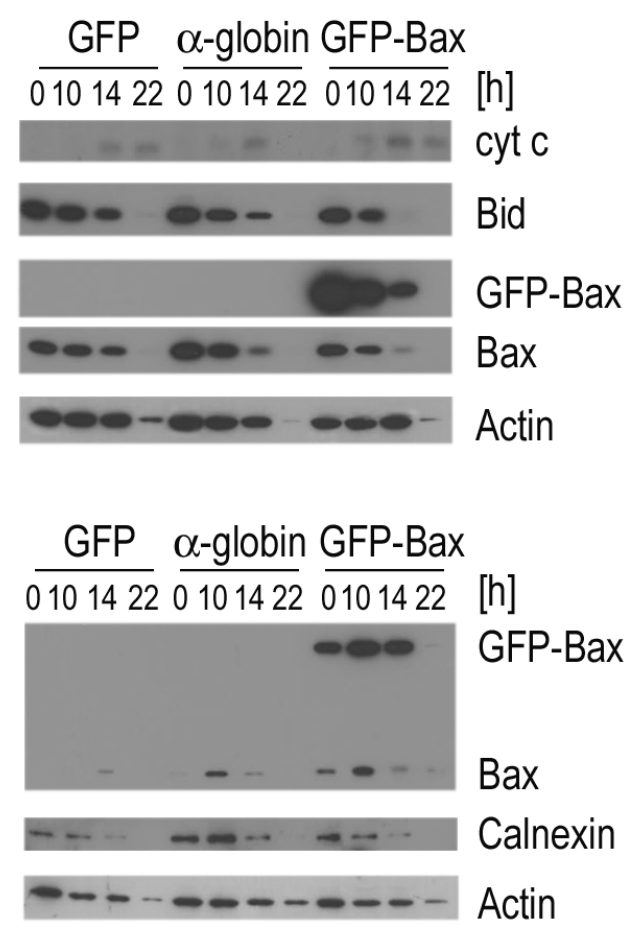

Figure 28: Translocation of Bax and cytochrome c, and cleavage of Bid occurs with faster kinetics in cells expressing GFP-alpha globin and GFP-Bax. FL5.12 cells transduced with GFP, GFP-alpha globin ( $\alpha$-globin), and GFP-Bax were grown in IL-3-free medium IL-3 for 0, 10, 14, and $22 \mathrm{~h}$. Cytosolic and membrane fractions were separated by ultracentrifugation. Proteins were analyzed by $12 \%$ SDS PAGE and Western blotting. The blot containing cytosolic proteins was successively probed with anti-cytochrome c, anti-Bid, anti-Bax, and anti-actin antibody (upper panel). The blot containing the membrane fraction was probed with anti-Bax, anti-calnexin and anti-actin (lower panel). Actin served as endogenous reference to normalize expression of cytosolic proteins.

Table 3: Translocation of Bax and cytochrome c, and cleavage of Bid is faster in cells expressing GFP-alpha globin and GFP-Bax.

\begin{tabular}{lcccc|cccc|cccc}
\hline \multicolumn{1}{c}{ GFP } & \multicolumn{1}{c}{ GFP- $\alpha-g l o b i n$} & \multicolumn{4}{c}{ GFP-Bax } \\
Time & $0 \mathrm{~h}$ & $10 \mathrm{~h}$ & $14 \mathrm{~h}$ & $22 \mathrm{~h}$ & $\mathrm{Oh}$ & $10 \mathrm{~h}$ & $14 \mathrm{~h}$ & $22 \mathrm{~h}$ & $0 \mathrm{~h}$ & $10 \mathrm{~h}$ & $14 \mathrm{~h}$ & $22 \mathrm{~h}$ \\
\hline Cyt C & 1.0 & 1.0 & 11.2 & 55.9 & 1.0 & 3.4 & 11.3 & 122 & 1.0 & 3.2 & 8.4 & 80.7 \\
Bid & 1.0 & 1.0 & 0.7 & 0 & 1.0 & 0.7 & 0.4 & 0.6 & 1.0 & 0.7 & 0 & 0 \\
Bax & 1.0 & 1.2 & 0.8 & 0 & 1.0 & 0.8 & 0.2 & 0.9 & 1.0 & 0.2 & 0 & 0 \\
\hline
\end{tabular}

The bands of the cytosolic proteins in figure 28 (upper panel) were quantitated using actin as endogenous reference to normalize expression. Expression values (n-fold) were calculated relative to expression at $\mathrm{t}=0 \mathrm{~h}$. 


\subsubsection{Antisense alpha globin decreases basal levels of alpha globin in FL5.12 cells and reduces induction of alpha globin under cytokine-deprived conditions}

Above we have shown that enrichment of ectopic GFP-alpha globin efficiently accelerated apoptosis in IL-3-deprived FL5.12 cells suggesting a role of alpha globin as a pro-apoptotic factor in PCD. On the other hand we asked whether silencing of alpha globin expression in FL5.12 cells could diminish apoptosis progression upon cytokine deprivation. We generated a retroviral antisense expression construct with an IRES for GFP and the reverse complement sequence of alpha globin containing a stop codon in the first codon. We demonstrated that antisense alpha globin efficiently reduced alpha globin protein. No band was detected in antisense alpha globin expressing cells as compared to GFP control cells in the presence of IL-3. In the absence of IL-3, however, we observed a slight increase of alpha globin protein (Figure 29).

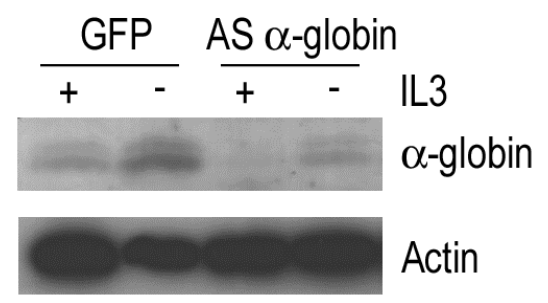

Figure 29: Antisense alpha globin reduces basal levels and upregulation of endogenous alpha globin protein in FL5.12 cells. FL5.12 cells transduced with GFP or antisense alpha globin (AS $\alpha$-globin) were grown in the presence or absence of IL-3 for $14 \mathrm{~h}$. Whole cell lysates were prepared and subjected to SDS PAGE and Western blotting. The blot was probed with anti-hemoglobin and anti-actin antibodies.

\subsubsection{Antisense alpha globin delays caspase-3 activation but not DNA fragmentation in FL5.12 cells}

In order to assess the biological effect of reduced alpha globin levels in FL5.12 cells we monitored the progression of apoptosis in cells expressing GFP or antisense alpha globin in the presence or absence of IL-3 for $14 \mathrm{~h}$. In parallel we evaluated the amount of TUNELpositive cells and assayed activation of caspase-3, respectively. DNA fragmentation was comparable and below $2 \%$ in both cell lines growing in the presence of cytokine (Figure 30a). We therefore conclude that expression of antisense alpha globin is well tolerated in FL5.12 cells. However, under IL-3-deprived conditions fragmentation of DNA was not delayed in antisense alpha globin expressing cells as compared to cells expressing GFP. GFP and antisense alpha globin transduced cells displayed $60 \%$ and 55\% TUNEL-positive cells, respectively (Figure 30b).

In contrast, experiments assessing the activity of caspase- 3 indicated that antisense alpha globin transduced cells exhibited reduced caspase- 3 activities as compared to GFP transduced cells lacking IL-3 (Table 4). Caspase-3 activity was reduced by a factor of approximately 2 in GFP-alpha globin expressing cells as compared to control cells. 
a

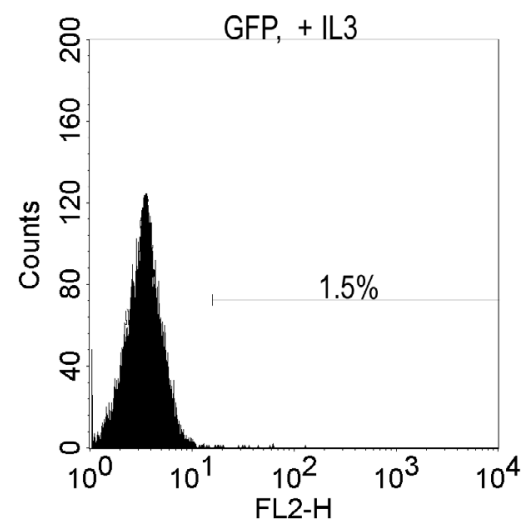

$b$

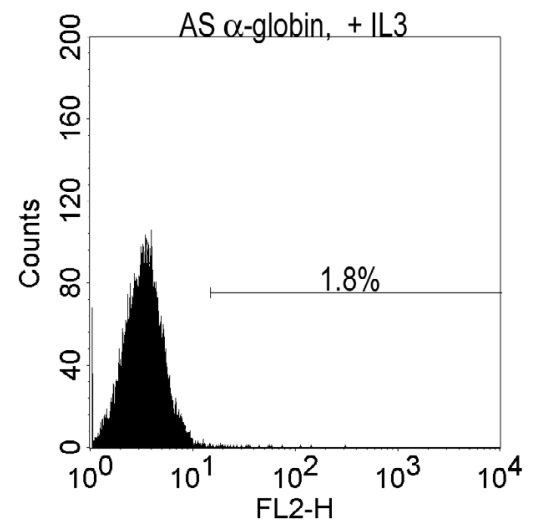

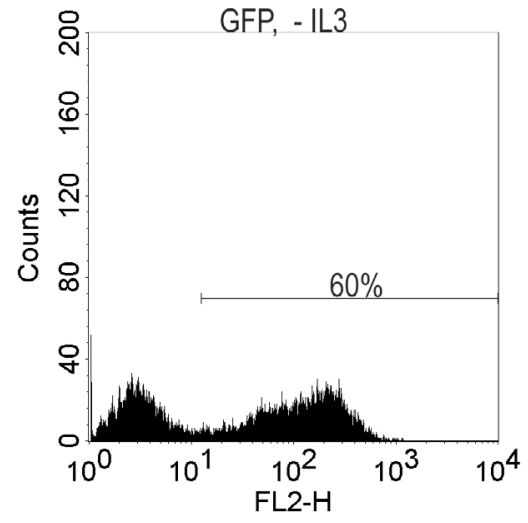

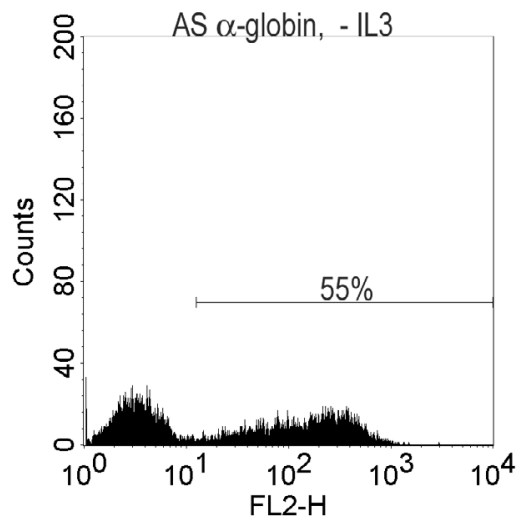

Figure 30: Antisense alpha globin does not prevent DNA fragmentation in FL5.12 cells. Cells expressing GFP (a) or antisense alpha globin (b) were grown in the presence or absence of IL-3 for $12 \mathrm{~h}$ and were subsequently subjected to TUNEL staining. TUNEL-positive cells were monitored using a FACS Calibur. The markers depict TUNEL-positive cells in percentage $(\%)$. The graphs represent one of three independent measurements.

Table 4: Antisense alpha globin prevents upregulation of endogenous alpha globin and reduces activation of caspase-3 in FL5.12 cells.

\begin{tabular}{|c|c|c|c|c|}
\hline & \multicolumn{2}{|c|}{ GFP } & \multicolumn{2}{|c|}{ AS alpha globin } \\
\hline & $+\mathrm{IL} 3$ & - IL3 & $+\mathrm{IL} 3$ & - IL3 \\
\hline \% adjusted volume actin & 21.0 & 11.0 & 15.5 & 15.2 \\
\hline$\%$ adjusted volume $\alpha$-globin & 1.4 & 2.1 & not present & 0.8 \\
\hline$\alpha$-globin (n-fold induction) & 1.0 & 2.8 & n.a & n.a \\
\hline $\begin{array}{l}\text { caspase- } 3 \text { activity (n-fold } \\
\text { induction) }\end{array}$ & 1.0 & 6.2 & 1.0 & 3.1 \\
\hline
\end{tabular}

FL5.12 cells, retrovirally transduced with GFP or antisense alpha globin were grown in the presence or absence of IL-3 for $14 \mathrm{~h}$. Whole cell lysates were subjected to SDS PAGE and Western blotting. The blot was probed with anti-hemoglobin and anti-actin antibodies (Figure 29) and signal intensities were analyzed. Alpha globin content and caspase-3 activities were normalized using actin as endogenous reference. Values obtained from cells growing with IL-3 were set as 1 . 


\subsection{Hematopoietic transcription factor GATA-2 promotes upregulation of alpha globin and cell death in FL5.12 cells}

\subsubsection{GATA-2 transcription factor is significantly upregulated in cytokine-deprived FL5.12 cells}

Next we addressed the question of which regulatory processes account for the upregulation of alpha globin in FL5.12 cells under death inducing conditions. Several mechanisms may achieve elevated alpha globin levels such as stabilization at transcript or protein level or transactivation by transcription factors. We focused on transcription factors as potential inducers of alpha globin transcription. To examine which transcription factors might be involved under IL-3 deprivation we compared the transcriptional response of FL5.12 cells growing with or without IL-3 for $0,1,8,16$, and $24 \mathrm{~h}$ using Affymetrix gene chip arrays. Transcription factors that were induced or repressed by more than two-fold were suggested to be differentially regulated and were considered further as candidate genes. We found that GATA-2 and EKLF were the only factors that were significantly upregulated, 3.3- and 2.3fold, respectively (Table 5). Overall-expression levels of GATA-2 were high while the levels of EKLF were low in FL5.12 cells. GATA-2 ensures maintenance and proliferation of immature hematopoietic progenitors and can induce both alpha and beta globin expression (Ohneda and Yamamoto, 2002). EKLF is implicated in adult beta globin gene expression, the developmental switch from fetal gamma to adult beta globin, and as a cofactor of GATA-1. Due to capacity reasons and the fact that beta globin is barely expressed in FL5.12 cells, which possibly indicates a minor role of beta globin, we did not study further the role of EKLF.

Next, we investigated whether other transcription factors, which are known to regulate transcript levels of alpha globin, were expressed and differentially regulated in death committed FL5.12 cells. In addition to transcription factors that were not differentially expressed such as ubiquitous factors CP-2 and trans-acting transcription factor Sp1, we found erythroid-specific transcription factors namely GATA-1, FOG-1, and NF-E2 to be upregulated, however, less than 2-fold (Table 5). Like EKLF, FOG-1 exhibits a GATA-1 binding site in the GATA-1 promoter and is believed to act as a coactivator and cofactor of GATA-1. NF-E2 is important for high level expression of alpha and beta globin (Martin et al., 1998). NF- $\kappa B$, a family of transcription factors mediating immune response and suppressing apoptosis, and implicated in cellular differentiations including erythropoiesis, was described to inhibit expression of alpha-like genes by repression of NF-E2 (Liu et al., 2003). In accordance, NF- $\mathrm{KB} 1$ (p50/p105) was slightly downregulated in FL5.12 cells exhibiting increasing levels of alpha globin. GATA-3, the third member of the GATA transcription factor family in hematopoietic tissues, was not expressed (Figure 31). In summary, several transcription factors with binding sites in globin as well as other erythroid genes were elevated. 
Table 5: Gene expression analysis of erythroid transcription factors in FL5.12 cells.

\begin{tabular}{|c|c|c|c|c|c|c|c|}
\hline & $\mathrm{Oh}$ & $1 \mathrm{~h}$ & $8 \mathrm{~h}$ & $16 \mathrm{~h}$ & $24 \mathrm{~h}$ & Regulation & Basal levels \\
\hline $\begin{array}{l}\text { GATA- } \\
1\end{array}$ & 1.3 & 1.2 & 1.8 & 1.9 & 1.2 & slightly up & low \\
\hline $\begin{array}{l}\text { GATA- } \\
2\end{array}$ & 1.1 & 2.0 & 2.9 & 3.3 & 2.8 & upregulated & high \\
\hline FOG-1 & 0.8 & 1.1 & 1.8 & 1.9 & 1.6 & slightly up & high \\
\hline EKLF & 1.0 & 1.3 & 2.3 & 2.3 & 1.8 & upregulated & low \\
\hline NF-E2 & 1.0 & 1.3 & 1.6 & 1.7 & 1.7 & slightly up & high \\
\hline CP-2 & 1.0 & 1.2 & 1.2 & 1.0 & 1.4 & $\begin{array}{l}\text { not } \\
\text { regulated }\end{array}$ & low \\
\hline Spl & 1.0 & 1.0 & 1.3 & 1.3 & 1.3 & $\begin{array}{c}\text { not } \\
\text { regulated }\end{array}$ & low \\
\hline NF- $\kappa B 1$ & 0.8 & 0.9 & 1.0 & 0.8 & 0.6 & slightly down & high \\
\hline
\end{tabular}

FL5.12 cells were grown in the presence or absence of IL-3 for $0,1,8,16$, and $24 \mathrm{~h}$. Total RNA samples were prepared according to the Affymetrix protocol. For analysis of relative expression values, the ratios of expression values of cells growing without IL-3 versus cells growing with IL-3 were evaluated for each time point and gene. Genes, differentially induced more than two-fold were termed "upregulated"; genes regulated between 1.5- and 1.9-fold were termed "slightly up" or "slightly down"; genes with expressions between 1and 1.4-fold were termed "not regulated".

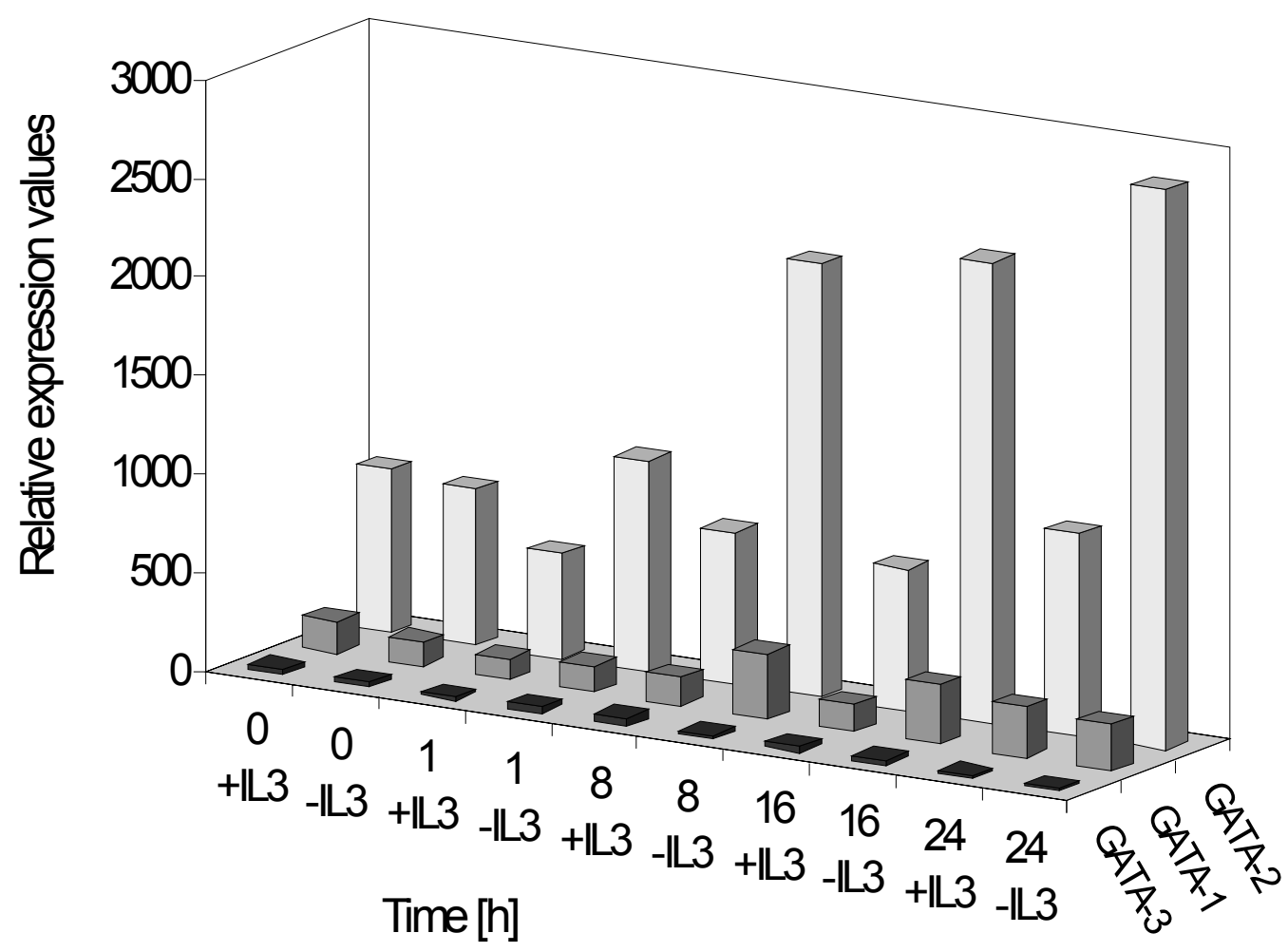

Figure 31: Gene expression analysis of erythroid transcription factors GATA-1, -2, and -3 in FL5.12 cells using Affymetrix gene chip 
arrays. FL5.12 cells were grown in the presence or absence of IL-3 for $0,1,8,16$, and $24 \mathrm{~h}$.

\subsubsection{GATA-1 and -2 are differentially expressed in various cell lines upon induction of apoptosis}

To confirm the results obtained from chip analysis and to further extend our investigations, we performed RT QPCR on FL5.12 cells treated with various cytotoxic compounds as well as on apoptosing NIH3T3, HeLa, and K562 cells. We found elevated GATA-1 and GATA-2 transcript levels in cytokine-deprived FL5.12 cells as seen in chip experiments. After $20 \mathrm{~h}$ in the absence of IL-3, GATA-2 transcript levels increased 2.8-fold, compared to 1.9-fold for GATA-1 transcript (Figure 32, upper panel). Treatment of FL5.12 cells with cisplatin or doxorubicin resulted in approximately 2.4-fold induction of alpha globin message, which, however, was not associated with GATA-2 or GATA-1 upregulation (Figure 32, upper panel).

GATA-1 was not expressed in NIH3T3 cells (Figure 32, middle panel). GATA-2 was expressed and strongly induced after treatment with cisplatin concomitant with alpha globin (Figure 32, middle panel). These results suggested that GATA-2, but not GATA-1, could be involved in induction of alpha globin expression in apoptosis.
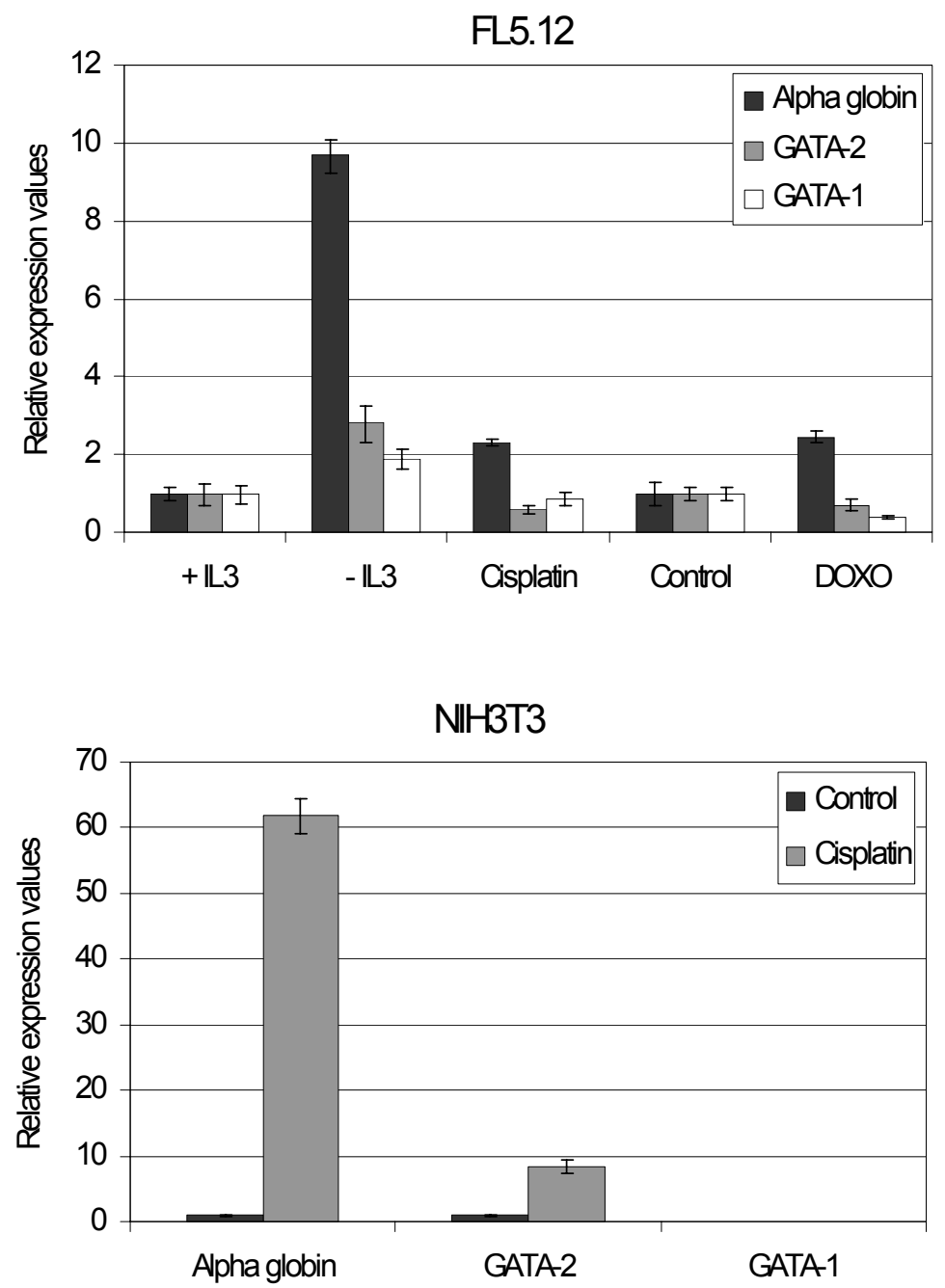


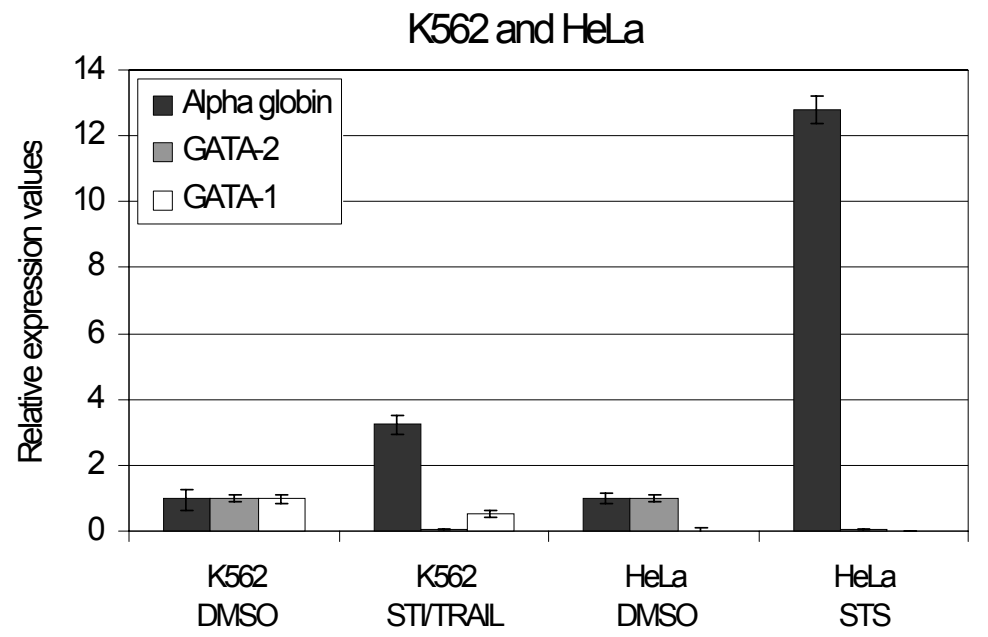

Figure 32: Expression of alpha globin, GATA-2, and GATA-1 mRNA in FL5.12, NIH3T3, K562, and HeLa cells undergoing apoptosis induced by different cytotoxic agents. Expression was quantitated by RT QPCR. Total RNAs were isolated from FL5.12 cells treated with $20 \mu \mathrm{M}$ cisplatin, grown with (+ IL3) or without IL-3 (- IL3) for $20 \mathrm{~h}$ or treated with $40 \mathrm{nM}$ doxorubicin or left untreated (Control) for $40 \mathrm{~h}$ (upper panel), NIH3T3 cells treated with $20 \mu \mathrm{M}$ cisplatin or left untreated for $30 \mathrm{~h}$ (middle panel), K562 simultaneously treated with $250 \mathrm{ng} / \mathrm{ml}$ TRAIL and $500 \mathrm{nM} \mathrm{STI571} \mathrm{(48} \mathrm{h)} \mathrm{or} \mathrm{left} \mathrm{untreated} \mathrm{(48} \mathrm{h),}$ and HeLa cells treated with $0.5 \mu \mathrm{M}$ STS $(6 \mathrm{~h})$ or left untreated $(6 \mathrm{~h})$ (lower panel). Each bar represents the average of triplicate measurements.

In contrast, K562 and HeLa cells, which have been found to upregulate alpha globin upon STI571/TRAIL and STS treatment, respectively, exhibited downregulation of GATA-2. GATA-1 levels were slightly down after $24 \mathrm{~h}$ (data not shown) and $48 \mathrm{~h}$ treatment with STI/TRAIL in K562 cells while GATA-1 was not expressed in HeLa cells (Figure 32, lower panel).

\subsubsection{NIH3T3, but not FL5.12 cells, ectopically expressing GATA-2, can be enriched}

Since GATA-2 was highly expressed and the most prominent upregulated transcription factor in FL5.12 cells and also present in NIH3T3 cells while GATA-1 was absent in NIH3T3 cells, we concentrated on GATA-2 for further investigations. We ectopically expressed and enriched GATA-2 in FL5.12 and NIH3T3 cells using retroviral transduction and FACS. We used an N-terminally GFP-tagged GATA-2 fusion construct, and a construct expressing GATA-2 with an N-terminal FLAG-tag and an IRES for GFP. Expression of GFP-positive FL5.12 cells was monitored $72 \mathrm{~h}$ after retroviral transduction before cells were sorted and again $48 \mathrm{~h}$ after sorting. Prior to sorting, GFP control cells exhibit 65\%, FLAG-GATA-2 40\%, and GFP-GATA-2 8\% GFP-positive cells (Figure 33). After one round of sorting 95\% of the control cells expressed GFP. In contrast, GFP- and FLAG-GATA-2 could not be 
successfully enriched by sorting. GFP-GATA-2 expressing cells displayed only $39 \%$ GFPpositive cells. FLAG-GATA-2 transduced cells died immediately and could not be analyzed further (Figure 33).
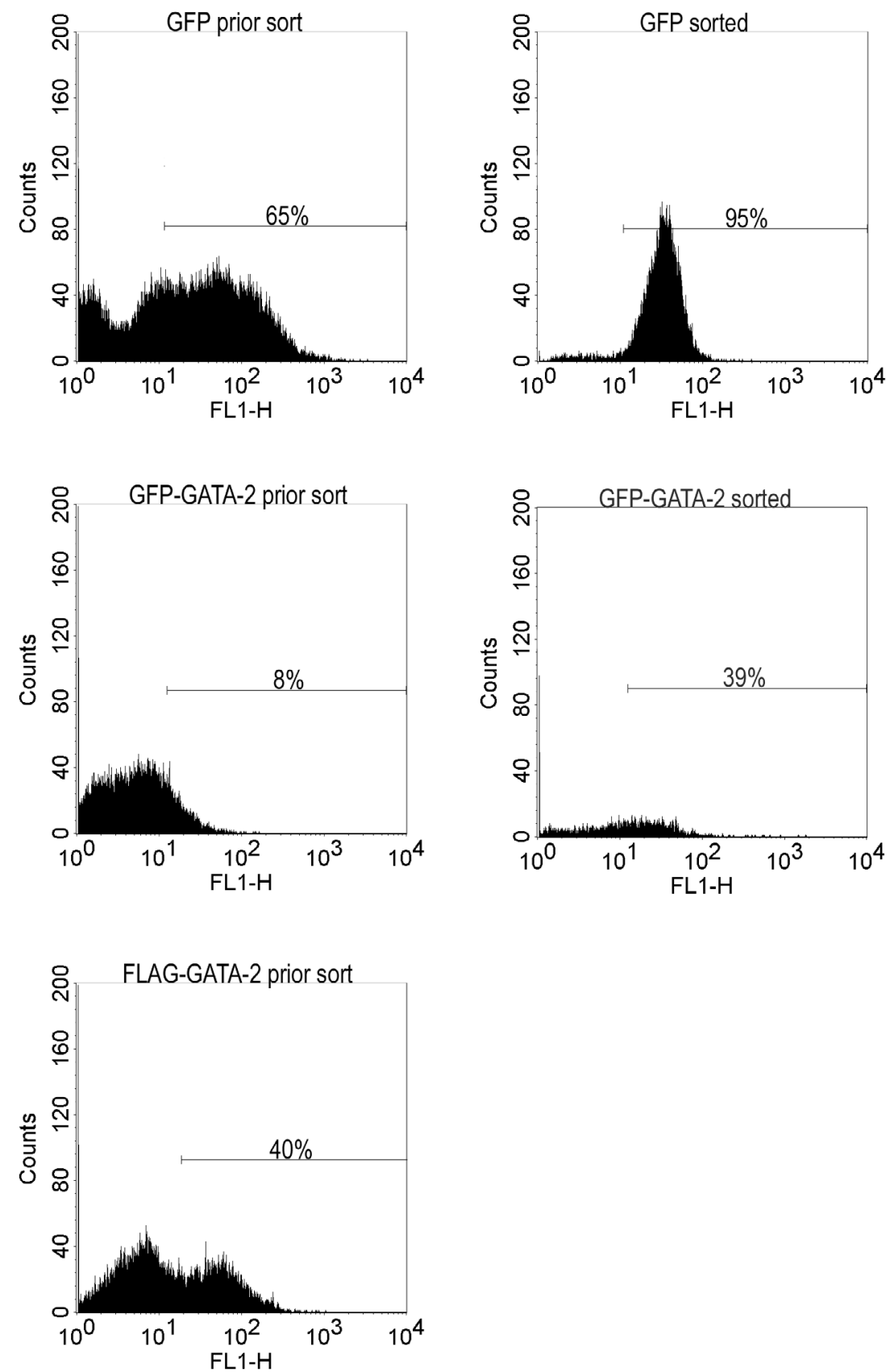

Figure 33: FL5.12 cells ectopically expressing GFP-GATA-2 cannot be enriched in FL5.12 cells. GFP expression profile of FL5.12 cells transduced with GFP, GFP-GATA-2 or FLAG-GATA-2 was monitored prior to or $48 \mathrm{~h}$ after sorting. The GFP signal was measured in the FL1-H channel of a FACS Calibur. The markers depict GFPpositive cells in percentage (\%). The graphs represent one of four independently performed transduction experiments. 
Expression of ectopic GATA-2 was examined at both transcript and protein level. RT QPCR was used to assess GATA-2 mRNA expression levels. In untransduced FL5.12 cells the threshold cycle was exceeded after 22 PCR cycles indicating that GATA-2 was significantly expressed (data not shown). GATA-2 transcript levels were moderately elevated in both ectopic FLAG- and GFP-GATA-2 expressing FL5.12 cells (Table 6).

In NIH3T3 cells endogenous GATA-2 was expressed at lower levels than in FL5.12 cells. The threshold cycle was detected after 26 PCR cycles. As NIH3T3 cells tolerated a second round of sorting, GATA-2 levels were dramatically elevated (Table 6).

Table 6: Ectopic GATA-2 is expressed in FL5.12 and NIH3T3 cells.

\begin{tabular}{|c|c|c|c|c|c|c|}
\hline & \multicolumn{6}{|c|}{ Relative expression ( $\mathrm{n}$-fold) } \\
\hline & GFP & Range & $\begin{array}{c}\text { GFP-GATA- } \\
2\end{array}$ & Range & $\begin{array}{c}\text { FLAG-GATA- } \\
2\end{array}$ & Range \\
\hline FL5.12 & 1.0 & $0.9-1.1$ & 12.1 & $10.9-13.4$ & 15.5 & $14.2-16.8$ \\
\hline $\mathrm{NIH} 3 \mathrm{T3}$ & 1.0 & $0.9-1.1$ & 401 & $365-441$ & 288 & $239-349$ \\
\hline
\end{tabular}

Total RNAs were isolated from FL5.12 and NIH3T3 cells retrovirally transduced with GFP, FLAG-GATA-2 or GFP-GATA-2. Transcript levels were measured using RT QPCR. Each value represents the average of triplicate measurements of two individual transduction experiments. The ranges indicate the variability around the average expression value.

\subsubsection{Ectopically expressed GATA-2 is localized to the nucleus in FL5.12 and NIH3T3 cells}

Next, we investigated GATA-2 protein expression and localization by Western blotting. Proteins of FL5.12 and NIH3T3 cells expressing GFP-GATA-2 were fractionated into cytosolic and nuclear fractions. Each fraction was subjected to SDS PAGE and immunoblotting. Unfortunately, none of the commercial GATA-2 antibodies detected GATA2 in our hands. Therefore we used anti-GFP antibody to detect the GFP-GATA-2 fusion protein. We detected GFP-GATA-2 exclusively in the nucleus of GATA-2, but not in GFP transduced cells (Figure 34a, b). 


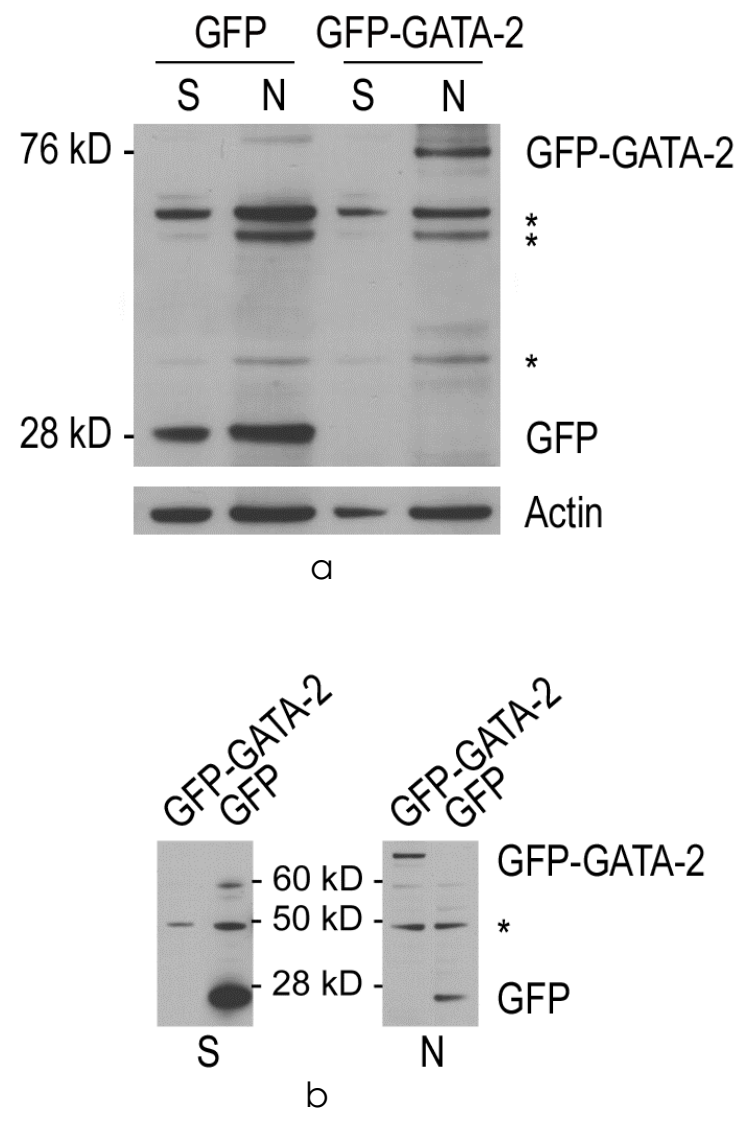

Figure 34: Ectopically expressed GATA-2 is localized to the nucleus in FL5.12 and NIH3T3 cells. FL5.12 (a) and NIH3T3 cells (b) were transduced with GFP or GFP-GATA-2 and sorted once using a FACS Calibur. Whole cell lysates were fractionated into soluble (S) and nuclear fraction $(\mathrm{N})$ and analyzed by SDS PAGE and Western blotting. Blots were probed with anti-GFP antibody. Background bands are marked with asterisks.

\subsubsection{Overexpression of GATA-2 induces cell death in FL5.12 cells and causes a transient stress phenotype in NIH3T3 cells}

Overexpression and enrichment of GATA-2 in FL5.12 cells were accompanied by cell death. This was consistently observed in several independent transduction experiments. Forty-eight hours after sorting for GFP or GFP-GATA-2, cells were stained with DAPI and analyzed by fluorescence microscopy. Cells overexpressing GATA-2 displayed typical characteristics of apoptosis such as membrane blebbing and loss of nuclear membrane integrity while control cells expressing high amounts of GFP were unaffected (Figure 36). Further, staining with DNA intercalating propidium iodide (PI) demonstrated that cells expressing GFP-GATA-2 lost plasma membrane integrity. Seventy-four percent stained PI-positive versus $3 \%$ in cells expressing GFP (Figure 35). 

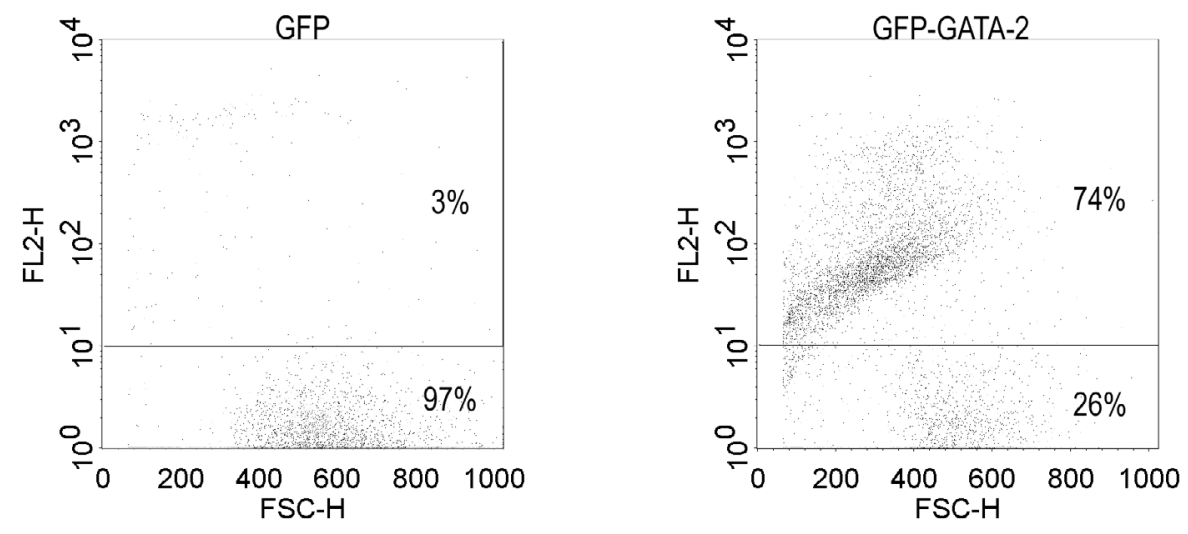

Figure 35: GATA-2 overexpression induces cell death in FL5.12 cells. FL5.12 cells were transduced with GFP or GFP-GATA-2. Forty-eight hours after sorting FL5.12 cells were stained with PI and PI-positive cells were analyzed in the FL2-H channel of a FACS Calibur.

In parallel we studied NIH3T3 cells retrovirally transduced with GFP-GATA-2. NIH3T3 cells did not die and nuclei remained intact upon expression of GATA-2, however, the cells displayed a severe stress phenotype with spindle-like cells as well as dramatically enlarged cells with reduced proliferation rate. GFP expressing cells, in contrast, tolerated retroviral transduction without phenotypical changes (Figure 37). The stress phenotype, however, was transient and disappeared two weeks after transduction and sorting. 

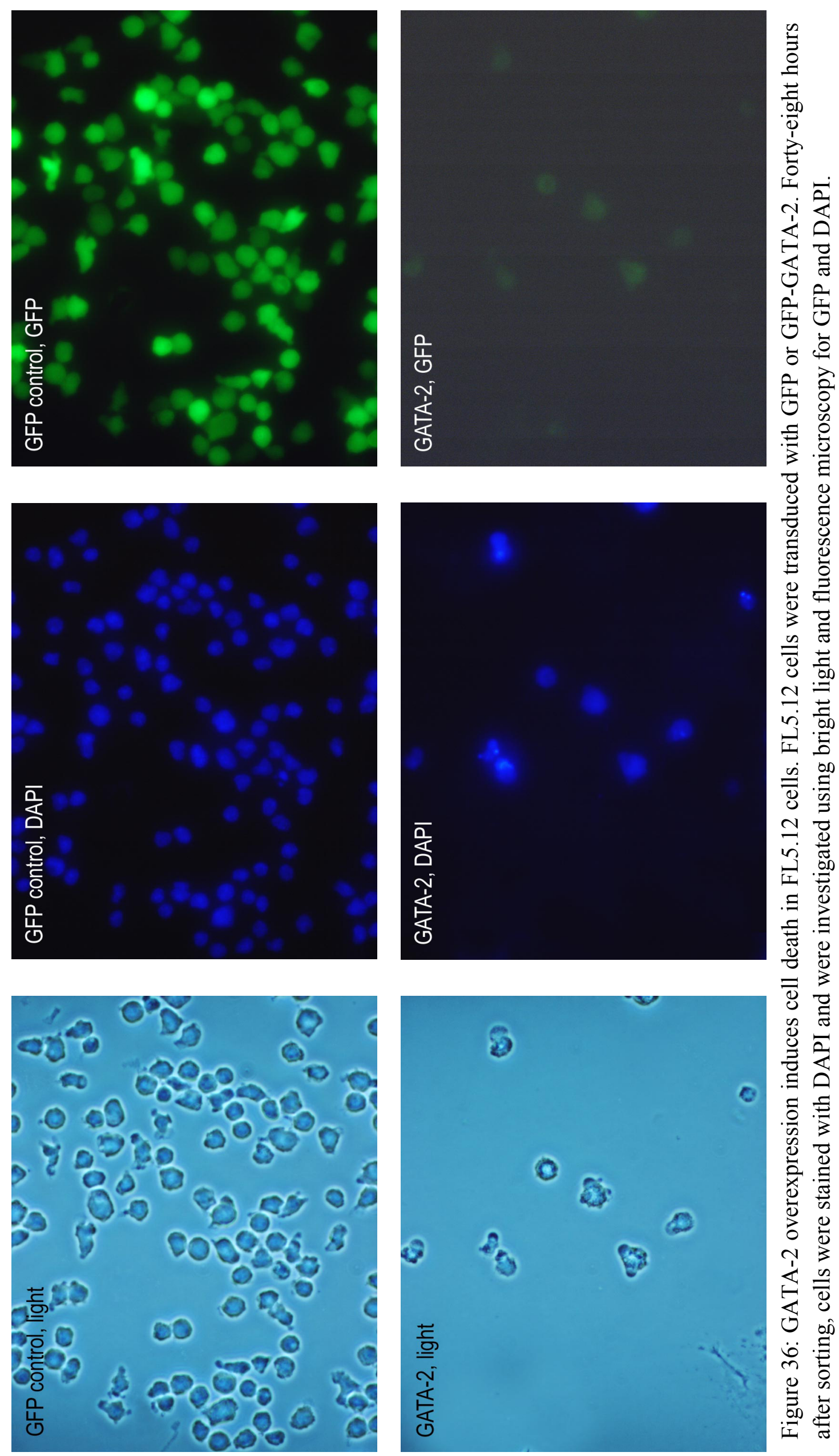

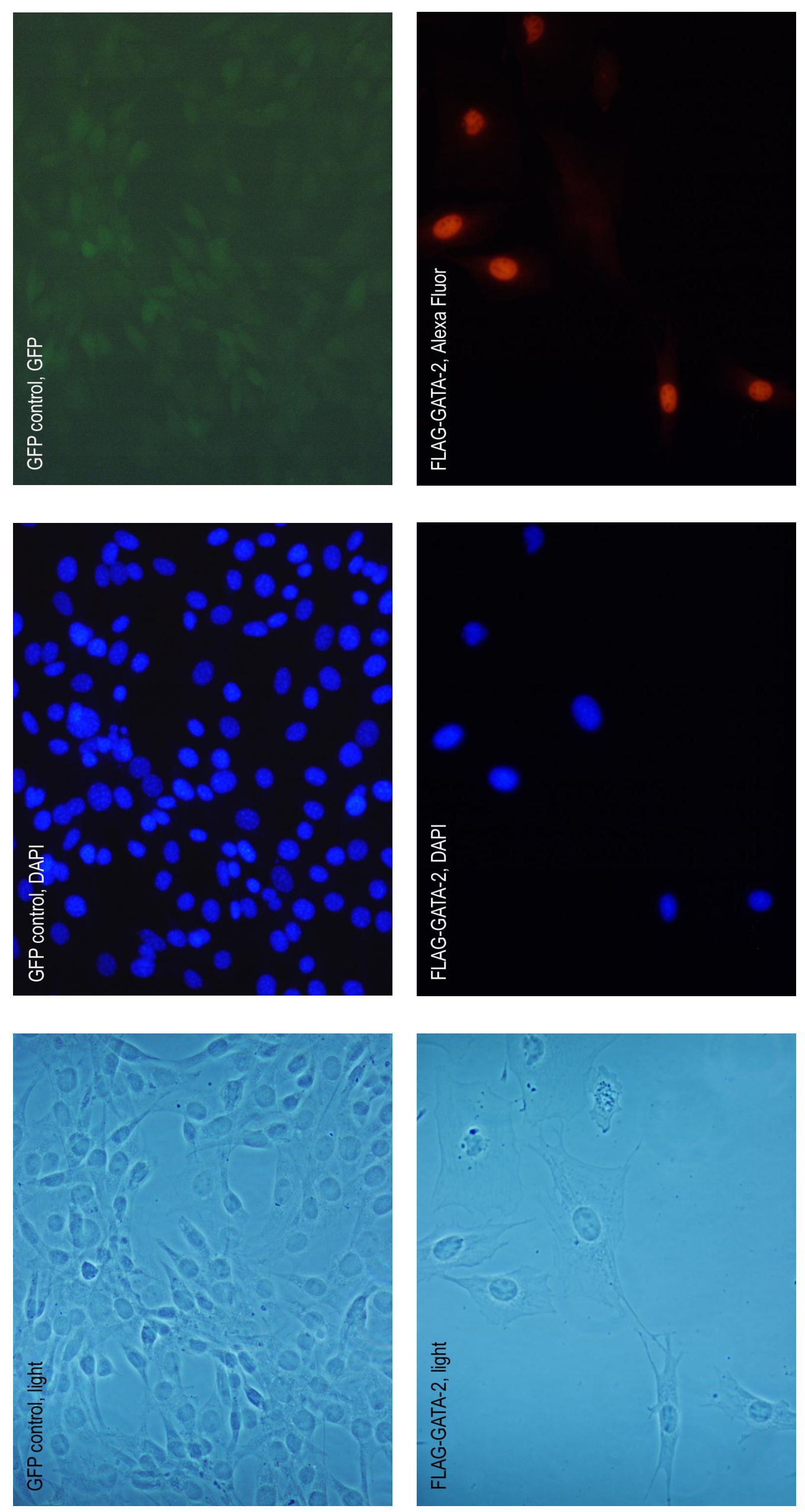


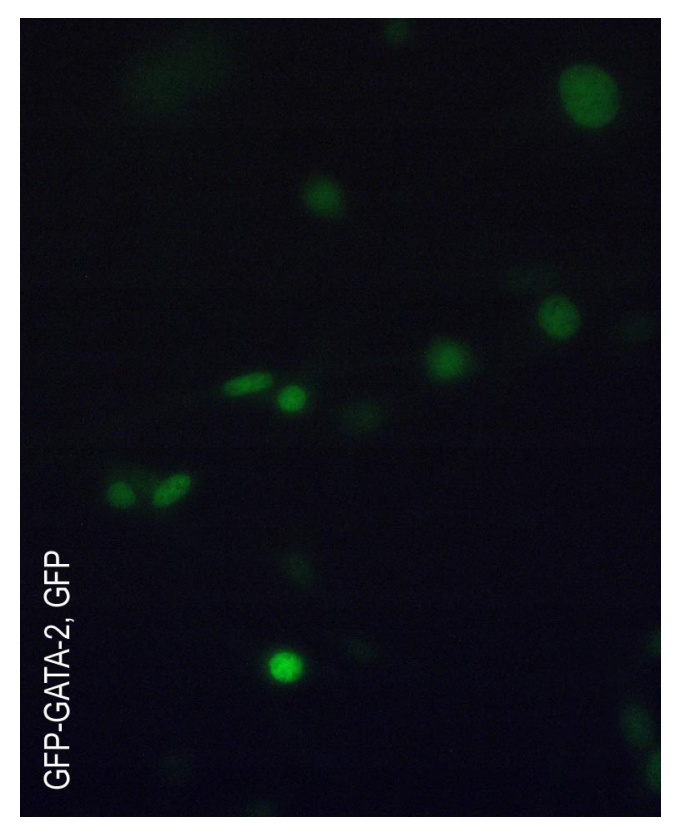

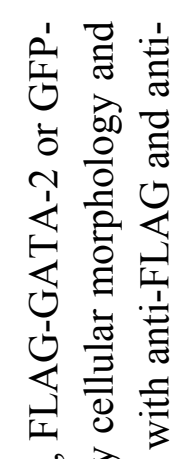

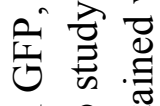

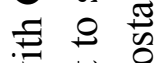

苛 营

元 등.

氙

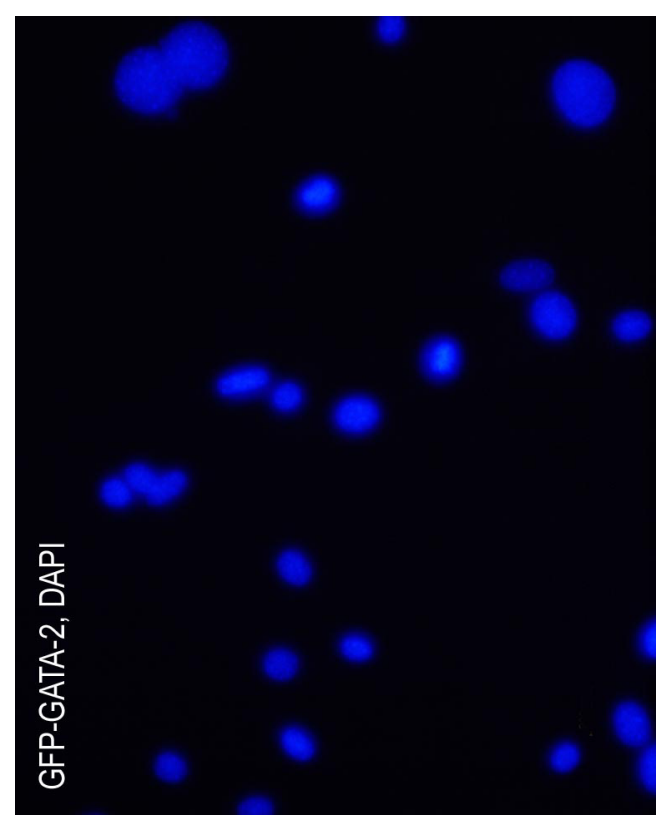

늠

3 च च

ষ

$m$ 政

毘离

Z 的客

$\dot{0}$ क ल

ల

U 묭

至完总

$\exists \exists$ 즌

范

룽 死

D

需

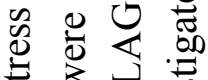

邱茳

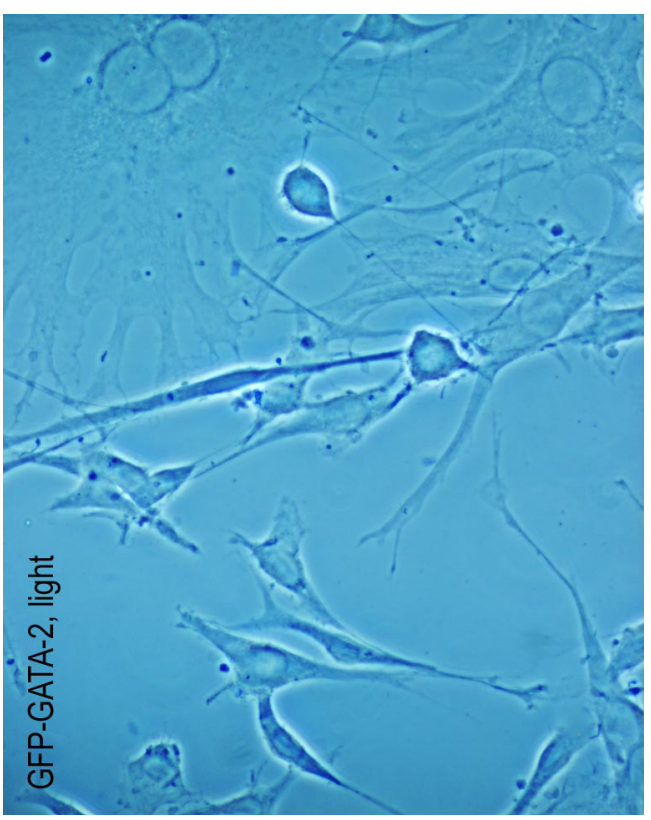

के

导

寻 营

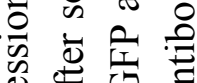

逍导 元

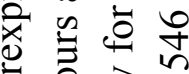

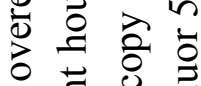

규유

这需

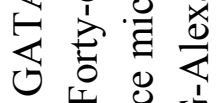

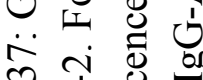

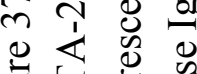

总芯芯芯芯 


\subsubsection{Ectopically expressed GATA-2 induces alpha globin expression in FL5.12 but not NIH3T3 cells}

To study whether the ectopically expressed GATA-2 was able to upregulate endogenous alpha globin in FL5.12 and NIH3T3 cells, we performed RT QPCR and Western blot analysis. FL5.12 and NIH3T3 cells were transduced with GFP, FLAG-GATA-2 or GFPGATA-2. As apoptosis occurred in FL5.12 cells after enriching GATA-2 by sorting, we processed cells immediately $48 \mathrm{~h}$ after transduction. In contrast, transduced NIH3T3 cells were sorted two times before RNA and proteins were isolated. For GATA-2 transduced NIH3T3 cells we did not detect elevated alpha globin levels (Figure 38, upper panel). FL5.12 cells, however, exhibited a clear response at alpha globin transcript and protein level when ectopically expressing GATA-2 (Figure 38, upper and lower panel). We further found that FL5.12 cells expressing FLAG-GATA-2 contained twice as much alpha globin message as cells expressing GFP-GATA-2. Above we have shown that GATA-2 levels were higher in FLAG-GATA-2 than in GFP-GATA-2 expressing cells (Table 6), which could explain the stronger induction of alpha globin message.

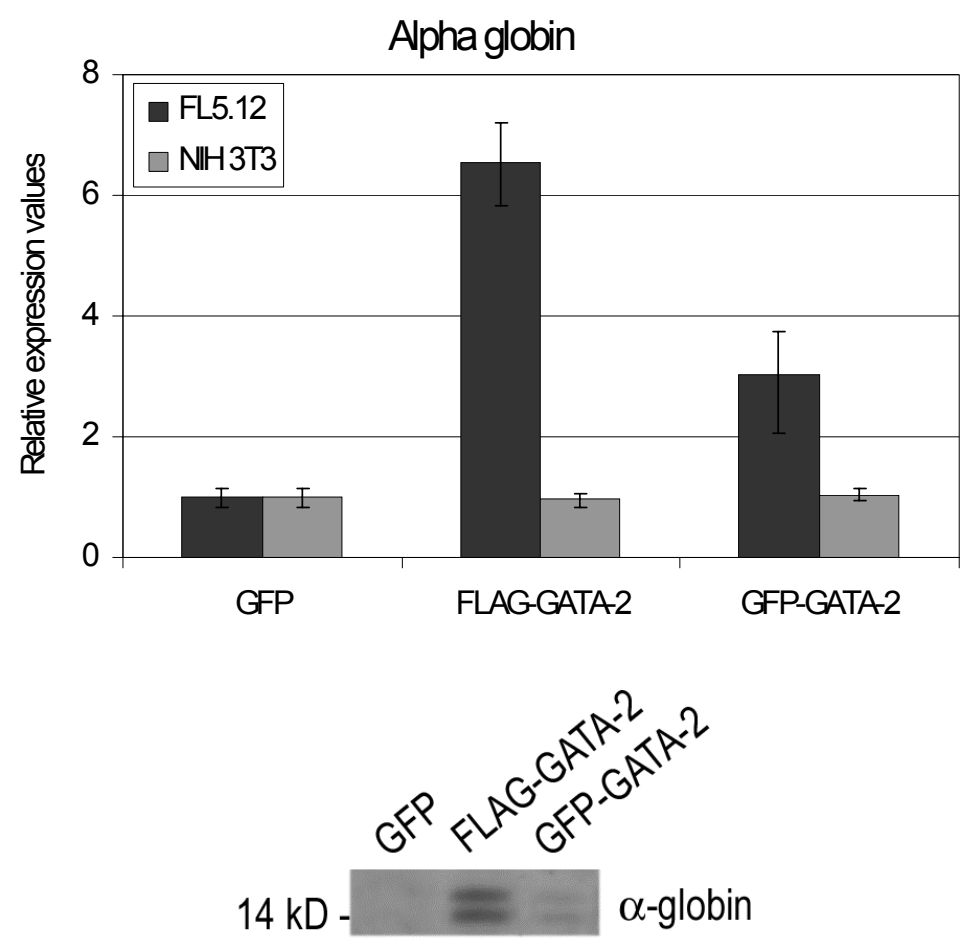

Figure 38: Overexpressed GATA-2 induces endogenous alpha globin at transcript and protein level in FL5.12 but not in NIH3T3 cells. FL5.12 and NIH3T3 cells were transduced with GFP, FLAG-GATA-2 or GFP-GATA2. FL5.12 cells were left unsorted while NIH3T3 cells were sorted twice to enrich GFP-expressing cells. RNA was isolated and alpha globin message measured by RT QPCR using alpha globin specific primers and probe (upper panel). Each bar represents the average of triplicate measurements. Whole cell lysates of FL5.12 cells were prepared and subjected to SDS PAGE and Western blotting. The blot was probed with anti-hemoglobin antibody (lower panel). 
The same dose-dependent effect was seen at protein level; FL5.12 cells expressing FLAGGATA-2 presented a more intensive band for alpha globin than GFP-GATA-2 expressing cells, while alpha globin levels were below detection in GFP transduced control cells (Figure 38, lower panel).

\subsubsection{Antisense GATA-2 prevents upregulation of GATA-2 and reduces upregulation of alpha globin under apoptotic conditions in FL5.12 cells}

We observed that GATA-2 promoted upregulation of alpha globin and induced apoptosis in FL5.12 cells. Hereupon we asked whether silencing of GATA-2 could prevent the increase of alpha globin and change the outcome of apoptosis in FL5.12 cells growing without IL-3. We generated a retroviral expression construct with an IRES for GFP and the reverse complement sequence of GATA-2 containing a stop codon in the first codon. FACS was used to enrich antisense GATA-2 expressing clones. First, we performed RT QPCR to test whether the antisense GATA-2 could counteract the upregulation of GATA-2 under cytokine-deprived conditions. Under growth conditions without IL-3 we found that antisense GATA-2 prevented the increase of GATA-2 compared to GFP control cells (Figure 39, upper panel).

Then we assessed expression of alpha globin in FL5.12 cells growing in the presence or absence of IL-3 for $14 \mathrm{~h}$. Using RT QPCR we observed that basal levels of alpha globin remained unchanged in FL5.12 cells transduced with antisense GATA-2 as was the case for cells transduced with GFP. Contrasting, in the absence of IL-3, upregulation of alpha globin in antisense GATA-2 expressing FL5.12 cells was reduced by approximately $45 \%$ as compared to control cells (Figure 39, middle panel). In parallel, we investigated alpha globin expression at protein level by Western blotting (Figure 39, lower panel). We consecutively probed the same blot with anti-hemoglobin and anti-actin antibody. The intensities of the bands were quantified and the amount of alpha globin was normalized using actin as endogenous reference. The increase of alpha globin protein was diminished by a factor of two in cells expressing antisense GATA-2, which was in line with our RT QPCR results (Table 7).

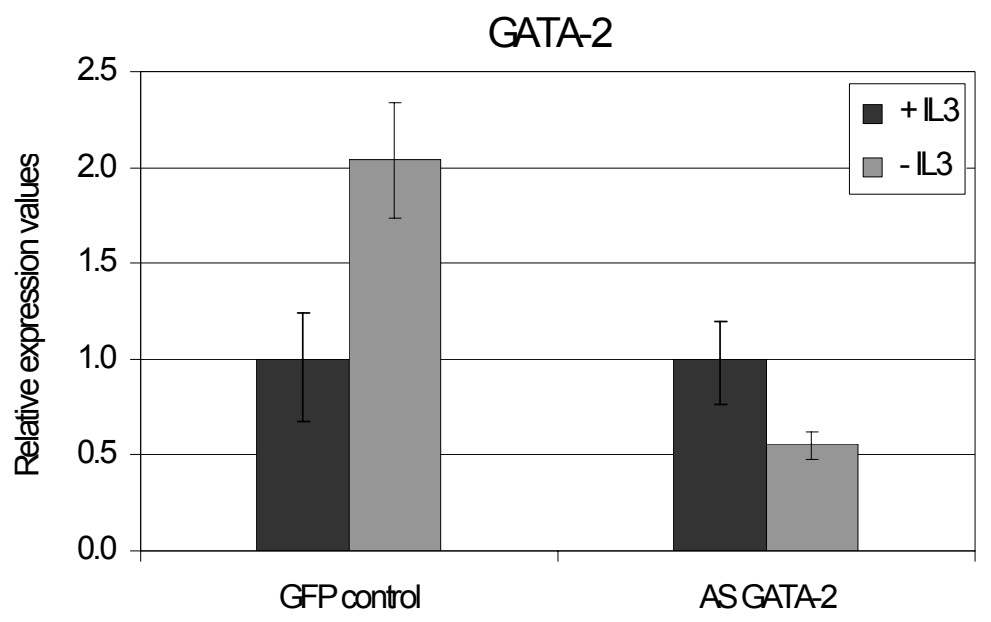




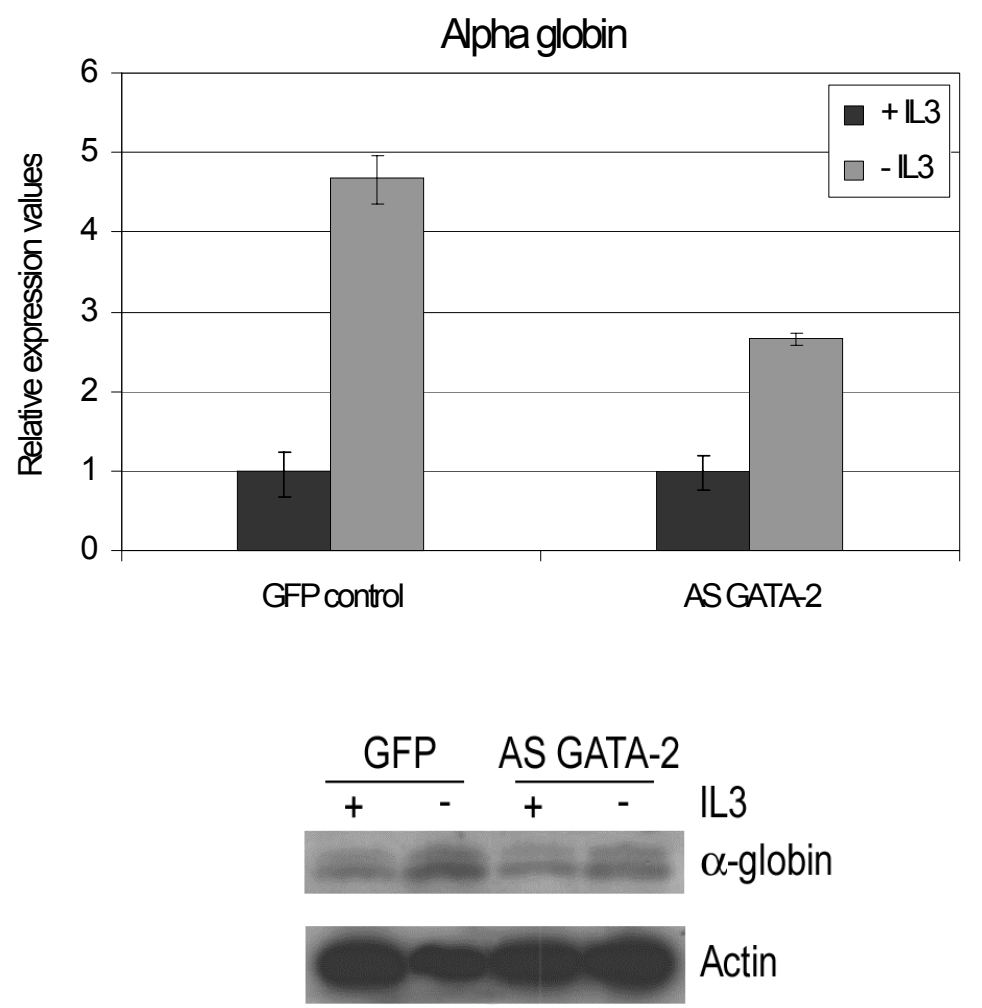

Figure 39: Antisense GATA-2 reduces upregulation of endogenous GATA-2 and alpha globin in FL5.12 cells. FL5.12 cells transduced with GFP or antisense GATA-2 were grown in the presence or absence of IL-3 for 14 h. $1.5 \times 10^{6}$ viable cells, as assessed by trypan blue staining, were subjected to RNA isolation and RT QPCR using GATA-2 (upper panel) and alpha globin (middle panel) specific primers and probes. Each bar represents the average of triplicate measurements. In parallel, whole cell lysates were prepared and subjected to SDS PAGE and Western blotting. The blot was probed with anti-hemoglobin and anti-actin antibodies (lower panel).

\subsubsection{Antisense GATA-2 delays DNA fragmentation in FL5.12 cells deprived of IL-3}

In parallel to RT QPCR and Western blotting, we examined the effect of antisense GATA-2 on progression of apoptosis in FL5.12 cells. FL5.12 cells ectopically expressing GFP or antisense GATA-2 were grown in the presence or absence of IL-3 for $14 \mathrm{~h}$ and subsequently subjected to TUNEL staining. Cells in the presence of IL-3 were TUNEL-negative indicating that expression of antisense GATA-2 was well tolerated in FL5.12 cells (Figure 40). Under IL-3-deprived conditions, however, fragmentation of DNA was significantly delayed in cells expressing antisense GATA-2 but not GFP. Antisense GATA-2 displayed 36\%, GFP 60\% TUNEL-positive cells during three independent experiments (Figure 40). Experiments assessing the activity of caspase- 3 underlined the protecting effect of antisense GATA-2 in the absence of IL-3: antisense GATA-2 transduced cells exhibited reduction of caspase-3 activities by $58 \%$ as compared to GFP transduced cells (Table 7 ). 

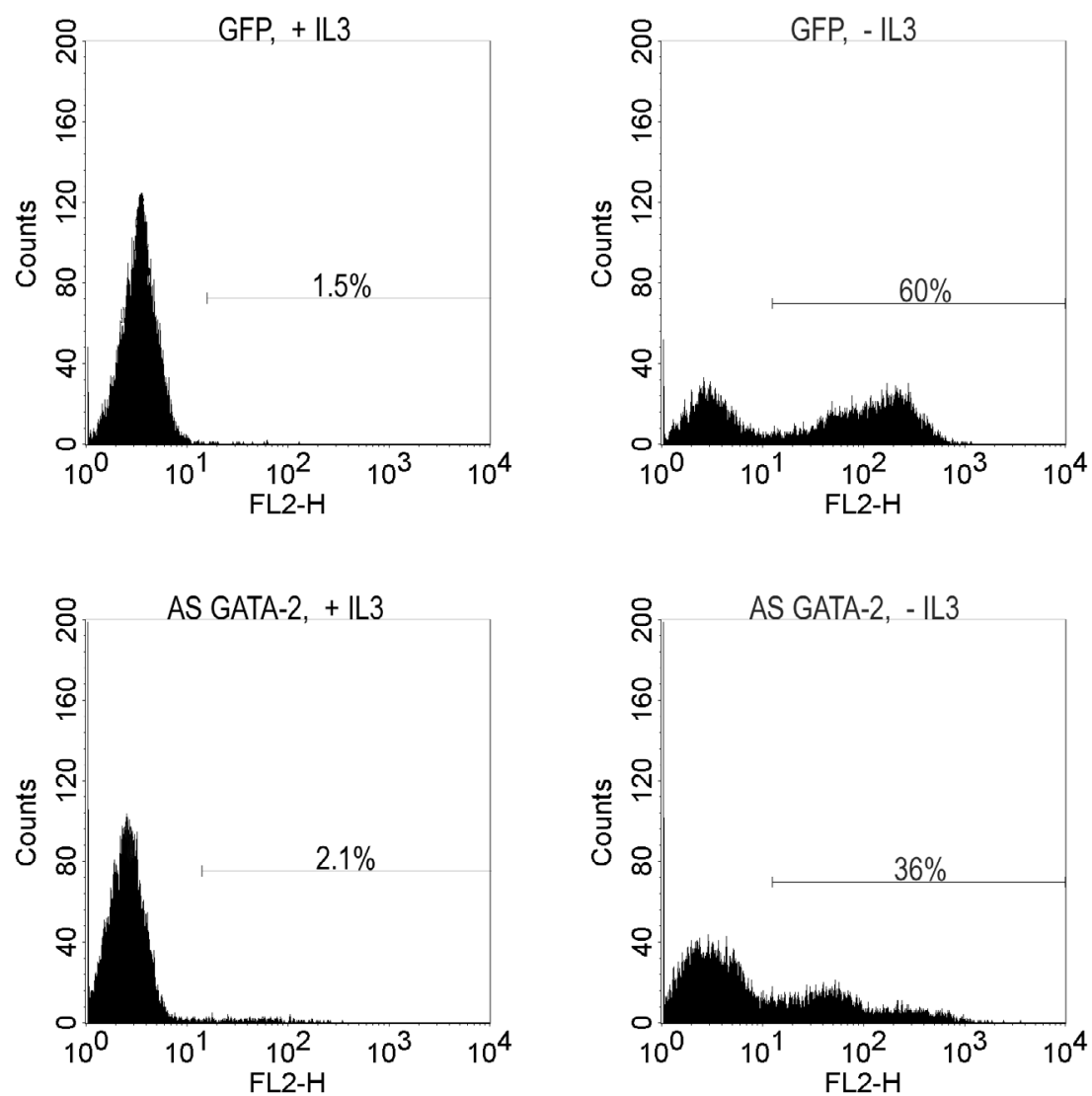

Figure 40: Antisense GATA-2 diminishes DNA fragmentation in cytokine-deprived FL5.12 cells. FL5.12 cells transduced with GFP or antisense GATA-2 were grown in the presence or absence of IL-3 for $14 \mathrm{~h}$. Cells were subjected to TUNEL staining. The markers depict TUNEL-positive cells in percentage (\%). Each graph represents triplicate measurements of three individual experiments.

Table 7: Antisense GATA-2 reduces upregulation of endogenous alpha globin and reduces activation of caspase-3 in FL5.12 cells.

\begin{tabular}{lcccc}
\hline & \multicolumn{2}{c}{ GFP } & \multicolumn{2}{c}{ AS GATA-2 } \\
& + IL3 & - IL3 & + IL3 & - IL3 \\
\hline \% adjusted volume actin & 21.0 & 11.0 & 15.2 & 19.9 \\
$\%$ adjusted volume $\alpha$-globin & 1.4 & 2.1 & 1.6 & 3.1 \\
$\alpha$-globin (n-fold induction) & 1.0 & 2.8 & 1.0 & 1.4 \\
$\begin{array}{l}\text { caspase-3 activity (n-fold } \\
\text { induction) }\end{array}$ & 1.0 & 6.2 & 1.0 & 2.6 \\
\hline
\end{tabular}

FL5.12 cells transduced with GFP or antisense GATA-2 were grown in the presence or absence of IL-3 for $14 \mathrm{~h}$. Whole cell lysates were subjected to SDS PAGE and Western blotting. The blot was probed with anti-hemoglobin and anti-actin antibodies (Figure 39, lower panel) and signal intensities were calculated. Alpha globin content and caspase-3 activities were normalized using actin as endogenous reference. Values obtained from cells growing with IL-3 were set as 1. 


\subsubsection{Overexpression or silencing of GATA-2 does not affect GATA-1 expression levels}

In erythroid cell development GATA-1 and GATA-2 are believed to follow auto- and crossregulatory processes. Especially GATA-2 has been shown to precede and transactivate GATA-1 expression (Visvader et al., 1995). In IL-3-deprived FL5.12 cells we also observed a significant upregulation of GATA-2, which preceded a slight increase in GATA-1. We asked whether ectopic GATA-2 induced its detrimental effect on FL5.12 cell viability at least to some extent via GATA-1. We compared endogenous GATA-1 transcript expression in FL5.12 cells expressing GFP or GFP-GATA-2. Both cell lines displayed comparable GATA-1 levels, i.e. overexpressed GATA-2 did not transactivate GATA-1 (Table 8). Similarly, antisense GATA-2 was found not to reduce basal GATA-1 expression (Table 8). In summary, alteration of GATA-2 levels by ectopic expression of sense and antisense GATA-2 had no impact on endogenous GATA-1 levels.

Table 8: Overexpression and silencing of GATA-2 does not affect GATA-1 expression levels.

\begin{tabular}{lcc}
\hline & $\begin{array}{c}\text { Relative } \\
\text { expression }\end{array}$ & range \\
\hline GFP & 1.00 & $0.83-1.20$ \\
GFP-GATA- & & \\
2 & 0.90 & $0.77-1.39$ \\
AS-GATA-2 & 0.84 & $0.78-0.91$ \\
\hline
\end{tabular}

GATA-1 transcript levels were measured in GFP, GFP-GATA-2 or antisense GATA-2 transduced FL5.12 cells growing in the presence of IL-3. Total RNAs were isolated and subjected to RT QPCR. Each value represents the average relative expression of triplicate measurements of two individual transduction experiments. Ranges indicate variability of the relative expression values.

\subsection{Beta globin exerts similar regulation trends as alpha globin}

As mentioned in chapter 4.1.2, we found beta globin to be expressed at its detection limit in FL5.12 cells. However, every time we detected regulation of alpha globin we also studied the expression of beta globin in parallel. Interestingly, basal beta globin mRNA expression levels of FL5.12 cells transduced with antisense alpha globin were reduced by a factor of five as compared to GFP transduced cells and even upregulation of beta globin in the absence of IL-3 was diminished by about 50\% (Table 9). GATA-2, known to bind to promotors and DNA regulatory sequences of several globin and erythroid genes, induced beta globin expression by a factor of five when overexpressed in FL5.12 cells (GFP-GATA-2, table 9). Antisense GATA-2 did not change basal beta globin expression. In the absence of IL-3 antisense GATA-2 caused a decrease of 29\% (Table 9), i.e. the effect was less pronounced than seen for alpha globin (Figure 39b). Summarizing, we confirmed that beta globin was concomitantly regulated with alpha globin but at approximately 200-fold lower expression levels. 
Table 9: Beta globin is regulated at low expression levels in FL5.12 cells.

\begin{tabular}{|c|c|c|c|c|}
\hline & \multicolumn{2}{|c|}{$+\mathrm{IL} 3$} & \multicolumn{2}{|c|}{ - IL3 } \\
\hline & Relative expression & range & $\begin{array}{l}\text { Relative } \\
\text { expression }\end{array}$ & range \\
\hline GFP & 1.00 & $0.83-1.20$ & 10.8 & $9.57-12.7$ \\
\hline AS $\alpha$-globin & 0.24 & $0.20-0.28$ & 1.59 & $1.49-1.69$ \\
\hline GFP-GATA-2 & 5.09 & $4.56-5.68$ & $n / a$ & $\mathrm{n} / \mathrm{a}$ \\
\hline AS GATA-2 & 0.86 & $0.76-0.97$ & 6.1 & $5.68-6.50$ \\
\hline
\end{tabular}

Cells expressing GFP, antisense alpha globin, GFP-GATA-2 or antisense GATA-2 were grown in the presence or absence of IL-3 for $12 \mathrm{~h}$. Total RNAs were isolated and subjected to RT QPCR. Each value represents the average relative expression of triplicate measurements of two individual transduction experiments. Ranges indicate variability of the relative expression values. 



\section{Discussion}

\subsection{Novel function of alpha globin promotes apoptotic cell death}

\subsubsection{Characterization of endogenous alpha globin in PCD}

The hematopoietic cell line FL5.12 is derived from murine fetal liver and possesses characteristics of lymphoid/myeloid precursor cells (Haughn et al., 2003). It expresses genes characteristic for non-differentiated pro B cells such as IL-3R, small amounts of class I MHC antigens and high densities of AA4 antigens (McKearn et al., 1985). The cells were not found to be induced to differentiate along the erythroid or myeloid lineage. FL5.12 can be maintained long-term in culture when provided with IL-3, whereas withdrawal of the cytokine induces apoptosis usually within $6 \mathrm{~h}$ post-starvation (McCubrey et al., 1989). In gene expression profiling of cytokine-deprived FL5.12 cells alpha globin, a gene predominantly expressed in erythrocytes, was shown to be dramatically upregulated (Brachat et al., 2000).

We confirmed that alpha globin was endogenously expressed and upregulated in the absence of IL-3. We showed that expression at transcript and protein levels correlated well as assessed by RT QPCR and immunoblotting, respectively, so we used RT QPCR, the more precise and reproducible method, to evaluate expression for most experiments.

Alpha globin mRNA levels remained stable during the first hour of IL-3 deprivation. We observed a strong incremental increase, however, during the following $7 \mathrm{~h}$. The maximal expression was reached after $12 \mathrm{~h}$, and was then followed by a gradual decrease after 16 and $20 \mathrm{~h}$. From this kinetics we conclude that alpha globin is induced more like delayed-response genes (Coyle et al., 2003), rather than immediate-response genes such as transcription factors c-fos or the early growth response genes (EGR) (Ahmed, 2004).

In addition to IL-3 deprivation, treatments with cisplatin and doxorubicin, two DNAintercalating compounds, or methotrexate, an inhibitor of dihydrofolate synthetase also induced upregulation of alpha globin in FL5.12 cells, however, at lower levels. These treatments all induce a block in DNA synthesis. In contrast, treatment with STS, a broadspectrum protein kinase inhibitor, camptothecin, a topoisomerase inhibitor inducing DNA strand breaks, or paclitaxel, a drug stabilizing microtubules, did not upregulate alpha globin expression (Brachat et al., 2002). Death receptor signaling induced by CHX/TNF alpha had the opposite effect, i.e. alpha globin expression decreased. Summarizing, in independent experiments we reproducibly observed that alpha globin was differentially expressed in FL5.12 cells depending on the apoptotic stimulus. We therefore propose that the changes in alpha globin levels are apoptosis-related, regulated events, rather than unspecific stress signals or artifacts associated with cell culture or sample preparation.

Besides alpha globin, no other globin or globin-like genes were found to be significantly expressed in FL5.12 cells. Myoglobin, zeta, epsilon and gamma globin were completely absent as assessed by gene expression analysis. Using gene chip arrays we found beta globin 
to be absent. However, after $16 \mathrm{~h}$ without IL-3 the signal increased, but remained below the detection level according to Affymetrix chip technology. By RT QPCR we were able to confirm that beta globin was expressed at the detection limit and that the expression was increased in apoptotic cells. From RT QPCR we estimated that beta globin was approximately 200 -fold lower expressed than alpha globin. We have to consider that this estimation is not precise but an approximation since the efficiency of the primers and probes for two different genes are not identical. In erythroid cells alpha and beta globin are expressed in a coordinate and balanced manner. We hypothesize that the expression of two globin chains, which are expressed at unequal level in FL5.12 cells, is still coupled to some extent in non-erythroid cells.

It has often been reported that undifferentiated cells undergoing apoptosis change their differentiation program down a different lineage to the one they have initially been committed to (Solary et al., 1993). This significant imbalance of alpha and beta globin indicated that the assembly of the two globin chains to form functional hemoglobin was rather unlikely in the pro B cell line FL5.12. We confirmed the absence of both hemoglobinization and concomitant differentiation down the erythroid lineage under cytokine-deprived conditions, using established hematological tests.

Very interestingly, we found that alpha globin was not associated with heme, the oxygenbinding prosthetic group of globin proteins. First, we demonstrated that ALA-S1 and PBGD, two genes involved in the synthesis of heme, were not upregulated in cytokine-derived FL5.12 cells. Second, endogenous heme levels remained constant during progression of apoptosis, although alpha globin increased by a factor of approximately 10 . In comparison, an increase of hemoglobin by a factor of nine in K562 treated with subtoxic concentrations of doxorubicin was paralleled by a 4-fold increase of heme indicating that the assay was sensitive. The heme level detected in FL5.12 cells is probably part of cellular cytochromes in mitochondria. We could further demonstrate that transcript levels of HO-1, which is a sensitive sensor of free heme, did not rise. We detected decreasing levels of alpha globin protein after 16 and $20 \mathrm{~h}$ of apoptosis and assumed that alpha globin was degraded. If alpha globin were in complex with heme, we would expect elevated amounts of free heme and therefore an increase of HO-1. Since alpha globin expressed in starved FL5.12 cells does not bind the prosthetic group in its hydrophobic pocket, it may have a different conformation than in erythrocytes or may bind another hydrophobic molecule instead.

In summary, we suggest that upregulation of alpha globin is involved in a specific apoptotic program and that the role of alpha globin in death-committed FL5.12 cells is different to its well-established role in oxygen transport in erythrocytes (SCHOLANDER, 1960).

Interestingly, we found that alpha globin expression and upregulation was also observed upon apoptosis induction in non-hematopoietic and differentiated cells such as NIH3T3, HeLa, and K562. As typical for an erythroid cell line, K562 expressed high levels of alpha globin, whereas NIH3T3 and HeLa cells had low endogenous levels. While alpha globin induction in the hematopoietic cells was moderate and about 3.5-fold, we detected a very strong increase in both differentiated cell lines. This suggests that upregulation of alpha globin is a common 
phenomenon in apoptosis and that especially cells having low basal levels upregulate alpha globin dramatically. While NIH3T3 cells showed alpha globin upregulation after cisplatin and STS treatment as observed in FL5.12 cells, HeLa cells displayed the exactly opposite regulation; STS but not cisplatin led to increased alpha globin levels. This suggests that upregulation of alpha globin depends not only on the apoptotic pathway, but also on the cell type.

Like FL5.12 also K562 cells expressed beta globin at the detection limit. As compared to alpha globin, which is expressed like a housekeeping gene in the CML cell line, beta globin was roughly $2^{12}$-fold less expressed. Thus, beta globin expression levels were also negligible in K562 cells. NIH3T3 and HeLa cells did not express beta globin at all. Hence, these observations do not support a novel function of beta globin in PCD.

Besides our findings suggesting a new role for alpha globin, other groups reported that alpha globin was implicated in processes other than oxygen transport. For instance, alpha globin has been found to be expressed in lens fibres in normal lenses and to be downregulated in the precataractous lens. Lenses undergo an apoptosis-like process during development, which results in removal of organelles and nuclei from their fibre cells. In cataractous lenses the organelles are not properly removed. This suggests a role of alpha globin in lens fibre differentiation (Wride et al., 2003). Also, Yoshida and colleagues found that extraerythrocytic hemoglobin played a novel role in wound healing. Hemoglobin and globin but not heme or protophorphyrin IX were able to stimulate plasminogen activator biosynthesis and to increase fibrinolytic activity in human fibroblasts (Yoshida et al., 2001).

The molecular weights of alpha and beta globin monomers of erythroid cells are identical, and both chains appear as a single $14 \mathrm{kD}$ protein band in Western blot analysis with the hemoglobin antibody we used (Spadaccini et al., 1998). In contrast, alpha globin in FL5.12 cells appears as a double band; the two bands differ by $0.5 \mathrm{kD}$ in size. The intensities of both bands increased when IL-3 was removed. The $14.5 \mathrm{kD}$ protein possibly contains some posttranslational, secondary modifications. We analyzed the alpha globin protein using the Scan Prosite database (http://www.expasy.org/tools/scanprosite/) to search for potential modification sites. Besides patterns with a high probability of occurence including sites for phosphorylation by protein kinase $\mathrm{C}$ and casein kinase II, N-myristylation and amidation, we found no specific sequences for posttranslational modification in alpha globin. Alternatively, alpha globin may be modified by acetylation on basic lysine residues. In recent years, protein acetylation has been found to be a wide-spread mechanism to control and modulate the activity of proteins. In addition to histones (Davie and Spencer, 1999), a growing number of proteins such as Sp-1 (Braun et al., 2001) and p53 (Appella and Anderson, 2001) have been shown to be targeted by histone acetyltransferases. However, since we were not able to define the nature of the $14.5 \mathrm{kD}$ band, we cannot fully exclude it being an unidentified cross-reactive protein, that is also upregulated under apoptotic conditions.

In cell fractionation experiments we found the smaller, $14 \mathrm{kD}$ alpha globin in the cytoskeleton of dying but not healthy FL5.12 cells. The cytoskeletal proteins containing alpha globin were obtained last from the fractionation protocol. Conversely, the fractions prepared earlier 
including membrane and nuclear proteins lacked alpha globin. We therefore suggest that the appearance of alpha globin in the cytoskeletal fraction does not reflect a simple dosedependent event. It has been reported that alpha globin increases membrane rigidity and apoptosis in pathologic erythrocytes as found in sickle cell disease or beta-thalassemia (Fortier et al., 1988; Advani et al., 1992). Under these conditions alpha globin was found to form complexes with spectrin, an actin-binding protein and the major component of the cytoskeleton. Whereas spectrin alpha and beta I are restricted to erythroid cells, spectrin alpha and beta II, referred to as fodrin alpha and beta, are present in other somatic cells (Coleman et al., 1989). Disturbance of membrane-cytoskeletal integrity could therefore be one possible function of alpha globin in PCD. Unfortunately, the commercially available hemoglobin antibody failed in immunoprecipitation, and we therefore lack direct evidence for alpha globin-spectrin interaction. Immunostaining using hemoglobin and spectrin antibody, respectively, and subsequent confocal microscopy could shed light on protein localization and possible protein-protein interaction.

Two years ago Kihm and Luzzatto et al reported that AHSP, a GATA-1 controlled erythroidspecific chaperone, forms a stable complex with alpha but not beta globin both in vitro and in vivo independently of heme (Kihm et al., 2002) (Luzzatto and Notaro, 2002). Endogenous AHSP was not expressed in FL5.12 cells as assessed by RT QPCR and immunoblotting. We ectopically expressed FLAG-tagged AHSP to evaluate whether AHSP was able to stabilize alpha globin in FL5.12 cells. We detected increased basal levels of the $14.5 \mathrm{kD}$ but not the 14 $\mathrm{kD}$ form of alpha globin. The viability of FL5.12 cells expressing AHSP was not different from the viability observed in GFP expressing cells both in the presence and absence of IL-3. We found, however, that FLAG-AHSP had a molecular weight of $14.5 \mathrm{kD}$ and was also detected by the hemoglobin antibody. We therefore could not conclude whether the increased band in FL5.12 cells was entirely due to overexpressed FLAG-AHSP or at least in parts due to alpha globin stabilized by AHSP. An AHSP expression construct of a different molecular size could be alternatively used in future experiments to test the alpha globin stabilizing properties of AHSP.

\subsubsection{Effects of overexpression and silencing of alpha globin in PCD}

Different from our earlier publication (Brachat et al., 2002), we now used an N-terminal GFPalpha globin fusion construct to overexpress alpha globin in FL5.12 cells instead of a construct expressing alpha globin with an N-terminal FLAG-tag and an internal ribosomal entry site for GFP. Using the latter construct sorting of GFP positive cells was easily achieved. We showed that ectopic FLAG-alpha globin was present both at genomic and transcript level indicating that this construct was integrated into the genome and transcribed. However, FLAG-alpha globin was never detected on Western blot indicating that the cells used mechanisms to silence exogenous FLAG-alpha globin protein. Furthermore, the progression of IL-3 deprivation-induced apoptosis of FL5.12 cells expressing FLAG-alpha globin was poorly reproducible. Phenotypes ranging from diminished to accelerated apoptosis were observed. In the current work we therefore chose a GFP-fusion construct, which ensured concomitant sorting of GFP and alpha globin. 
High expression of GFP-Bax, a potent cell death inducer in many cell types, was obtained without sorting and was tolerated without cell death; only few spontaneously dying cells with GFP-Bax localized in mitochondria were observed. It has been reported earlier that Bax accumulation was readily achieved in FL5.12 cells in the presence of IL-3 (Zhang et al., 1998). In contrast to GFP-Bax, enrichment of GFP-alpha globin was difficult in FL5.12 cells, necessitating two rounds of sorting. Ectopic expression of GFP-alpha globin in the presence of IL-3 was not accompanied by measurable apoptosis though. Even the proliferation rate of cells expressing GFP-alpha globin or GFP as control was comparable. Sporadically, we detected cells expressing higher levels of GFP-alpha globin and forming aggregates. We assume that clones expressing low levels of GFP-alpha globin were less affected by precipitated alpha globin and that these clones preferentially expanded. Excessive and nonstabilized alpha globin is known to be harmful for erythrocytes and to induce apoptosis in erythroid precursors from patients with beta-thalassemia. Precipitation of alpha globin is assumed to be triggered by oxidation of heme-bound iron initiating structural perturbations that culminate in protein denaturation (Fessas et al., 1966) (Yuan et al., 1995). However, we found that FL5.12 cells overexpressing GFP-alpha globin did not display elevated heme levels indicating that ectopic alpha globin was not associated with the prosthetic group. We therefore conclude that malfolded rather than oxidized GFP-alpha globin precipitates in FL5.12 cells.

Three kinase signaling cascades, the Jak/STAT, the Ras/Raf/MEK/ERK and the PI3K/Akt pathways, are important survival signaling pathways in FL5.12 cells triggered by IL-3. These pathways are interwoven and together they control cell death by phosphorylating several apoptotic and cell cycle regulatory proteins like caspase-9 (Cardone et al., 1998), BAD (Datta et al., 1997) and FKHRL1 (Brunet et al., 1999). The survival signaling cascade is immediately interrupted when IL-3 is removed, which leads to subsequent dephosphorylation, activation of pro-apoptotic factors, and release of cytochrome c from mitochondria.

Upon IL-3 removal, FL5.12 cells expressing GFP-alpha globin displayed increased internucleosomal DNA fragmentation as assessed by TUNEL staining. Higher alpha globin enrichment in the sorted cells, was accompanied by a faster apoptotic process, indicating a dose-dependent effect of alpha globin on apoptosis. Expression of equivalent levels of GFPalpha globin and GFP-Bax in FL5.12 cells resulted in a comparable decrease of viability in the absence of IL-3. The fact that endogenous alpha globin is strongly upregulated in cytokine-deprived FL5.12 cells and that ectopic alpha globin significantly accelerates PCD, clearly demonstrate the pro-apoptotic feature of alpha globin.

Interestingly, after deprivation of cytokine we observed that endogenous levels of alpha globin increased less in FL5.12 cells expressing GFP-alpha globin than in control cells. This suggests that the transduced GFP-alpha globin accelerates apoptosis and exerts negative feedback inhibition on synthesis of endogenous alpha globin.

To elucidate which apoptosis pathways are specifically promoted by alpha globin in FL5.12 cells, we investigated the role of caspases more closely. It was reported earlier that cell death in FL5.12 cells induced by IL-3 deprivation was independent of caspases (Bojes et al., 1999). 
In contrast, we demonstrated that pan caspase inhibitor, zVAD-fmk, moderately but significantly delayed apoptosis triggered in the absence of cytokine. However, since we could not prevent apoptosis completely even with high doses of zVAD-fmk, we suggest that caspase-independent processes may be indeed involved. We further found that inhibition of apoptosis by zVAD-fmk was less efficient in cells expressing GFP-alpha globin or GFP-Bax than in GFP expressing cells. This led to the conclusion that either caspase-independent processes were involved, or that the levels of active caspases were too high to be efficiently neutralized by the caspase inhibitor in FL5.12 cells transduced with GFP-alpha globin or GFP-Bax.

It has not been investigated so far which caspases are particularly activated in IL-3-deprived FL5.12 cells. We therefore analyzed the activities of caspase- $2,-3,-8$, and -9 using a caspaseprofiling plate to shed light on the caspases involved, especially in GFP-alpha globin accelerated cell death. Abrogated phosphorylation of caspase-9, as well as increased activity of the mitochondrial pathway was reported to cause strong caspase- 9 activation (Cardone et al., 1998). In the apoptosome, pro-caspase-9 becomes cleaved upon assembly with cytochrome c, Apaf-1, and ATP or dATP (Zou et al., 1999).

Caspase- 8 is primarily involved in death receptor signaling. Depending on the intensity of the stimulus, caspase- 8 triggers apoptosis via the mitochondrial amplification pathway (Kuwana et al., 1998) or directly through downstream caspase cascades (Scaffidi et al., 1998). Recently, the PI3K/Akt survival signaling pathway was shown to regulate the expression of FLIP, a natural inhibitor of caspase- 8 in endothelial cells (Skurk et al., 2004). This points to a potential connection between caspase- 8 and Akt signaling and could explain activation of caspase- 8 upon cytokine deprivation in FL5.12 cells.

Caspase-2 is widespread and highly expressed in hematopoietic cells (Johnson, 1998) and can trigger apoptosis via the non-mitochondrial and the mitochondrial pathway with cytochrome $\mathrm{c}$ release. There is growing evidence that caspase-2 plays a central role in IL-3 dependent signaling. E.g. antisense caspase- 2 has been reported to prevent apoptosis induced by cytokine removal in IL-3 dependent FDC-P1 cells, suggesting an essential role of caspase-2 in cell death induced by cytokine deprivation (Kumar, 1995). So far it has not been fully elucidated which mechanisms lead to caspase-2 activation (Lassus et al., 2002; Troy and Shelanski, 2003). In cell-free systems, however, caspase- 9 has been shown to be required for the activation of caspase-2 and other downstream caspases (Slee et al., 1999).

Caspase-3 is the major executioner caspase. Data exist supporting the idea that pro-caspase-3 resides in the mitochondrial inner membrane space and is released together with cytochrome c upon disruption of mitochondria (Blalock et al., 1999). Besides cleavage of at least 42 of 58 known caspase substrates, caspase- 3 can activate caspase-2, -6, -7, and -9 and autoactivate itself (Porter and Janicke, 1999).

In our profiling experiments, caspase- 3 and 2 were dramatically activated. Caspase- 8 was processed to some extent, while caspase-9 seemed to be very weakly activated. We found that GFP-Bax and GFP-alpha globin expressing cells displayed higher activities of all caspases 
tested than control cells. Further, both the dose-dependent effect of differentially enriched alpha globin and the protecting properties of Bcl- $\mathrm{x}_{\mathrm{L}}$ in alpha globin expressing cells were confirmed as seen in TUNEL staining. However, the assessment of caspase activity via cleavage of caspase substrates provides only crude information, since the substrates are unspecific. On the other hand, it is well accepted that caspases, which appear to be cleaved in immunoblots, are activated. Therefore, we extended our investigations on Western blot analysis. We observed strong activation of caspase- 3 and weak activation of caspase- 8 as seen on the profiling plate. In contrast, caspase- 2 was only slightly processed as assessed in immunoblots. We therefore conclude that caspase-3 accounted for the cleavage of the caspase-2 substrate in the profiling experiment to a significant extent. Different from our profiling results we found strong activation of caspase- 9 suggesting that the assay conditions for caspase-9 in the profiling experiment were suboptimal. Summarizing the data from both experiments, GFP-Bax and GFP-alpha globin significantly promote the activation of caspase- 3 and -8 . Caspase- 9 activation is probably promoted by both proteins, while no conclusions can be made in terms of caspase- 2 .

Our results derived from the caspase experiments indirectly suggest that the mitochondria probably play an important role in death signaling in FL5.12 cells upon removal of IL-3. For direct evidence we investigated $\mathrm{Bid}, \mathrm{Bax}$, and cytochrome $\mathrm{c}$, three pro-apoptotic proteins with functions in mitochondrial signaling. Full-length Bid is inactive and resides in the cytoplasm. Upon apoptosis induction, two caspases are known to cleave and activate Bid, caspase- 2 and -8. Both caspases were found to be activated in IL-3-deprived FL5.12 cells in our experiments. tBid is known to translocate to mitochondria and promote conformational change, oligomerization and recruitment of Bax to the mitochondria (Eskes et al., 2000). Subsequently cytochrome c is released to assemble in the apoptosome (Luo et al., 1998).

In FL5.12 cells transduced with GFP-alpha globin or GFP-Bax these three essential steps in the mitochondrial pathway were enhanced as compared to control cells; we detected disappearance of full-length Bid from the cytosol, Bax translocation from the cytosol to mitochondria, and release of cytochrome c. In GFP-Bax overexpressing cells, both endogenous and ectopic GFP-Bax translocated from the cytosol to the membrane fraction. We therefore suggest that overexpressed, translocated Bax was the predominant factor enhancing the release of cytochrome $\mathrm{c}$ in the cell death process. In contrast, ectopic alpha globin did not translocate to mitochondria although all markers for enhanced mitochondrial signaling were present.

We have shown that Bcl- $\mathrm{x}_{\mathrm{L}}$ efficiently inhibited apoptosis accelerated by alpha globin. Both caspase activation and DNA fragmentation were found to be blocked in FL5.12 cells coexpressing alpha globin and $\mathrm{Bcl}-\mathrm{x}_{\mathrm{L}} \cdot \mathrm{Bcl}-\mathrm{x}_{\mathrm{L}}$ is known to counteract the pro-apoptotic effects of Bax and to prevent cytochrome c release induced by ectopically expressed Bax (Zha et al., 1996), (Finucane et al., 1999). We have shown earlier that also Bcl-2 acts as a potent inhibitor of alpha globin-induced apoptosis in FL5.12 cells (Brachat et al., 2002). Anti-apoptotic Bcl-2 resides in membranes of mitochondria, endoplasmatic reticulum and the nuclear envelope, and prevents Bax translocation to the mitochondria and subsequent cytochrome c release 
(Murphy et al., 2000). Thus Bcl- $\mathrm{x}_{\mathrm{L}}$ is now the second member of the Bcl-2 family that counteracts the pro-apoptotic effect of alpha globin. Since both anti-apoptotic proteins neutralize apoptosis mainly at the mitochondrial level our observations emphasize the importance of alpha globin in stimulation of the mitochondrial apoptosis pathway.

An approach to further elucidate the importance of alpha globin in signaling apoptosis was through silencing of endogenous alpha globin using an antisense alpha globin construct. It has been reported that the generation of double stranded RNA can trigger antiviral defense mechanisms. In this context the double-stranded RNA-dependent protein kinase (PKR) has been reported to promote subsequent apoptosis (Gil and Esteban, 2000). However, we did not observe any changes in cell viability or proliferation in transduced FL5.12. Antisense alpha globin reduced basal alpha globin protein levels under normal growth conditions very efficiently, but could not completely prevent its upregulation in the onset of apoptosis. The phenomenon of incomplete silencing is a common problem associated with antisense approaches. It suggests either insufficient compensation of upregulated alpha globin message by the antisense construct or stabilization of alpha globin at protein level as a mechanism to overcome silencing. Under cytokine-deprived conditions, antisense alpha globin was not able to slow down apoptosis as assessed by TUNEL staining. In contrast, caspase-3 activity was reduced in antisense alpha globin transduced FL5.12 cells. Probably even the small incremental increase of alpha globin was sufficient to promote the apoptotic process.

Having shown that overexpressed alpha globin enhanced cell death in FL5.12 cells growing without IL-3, we asked whether overexpressed alpha globin also promoted PCD in NIH3T3 fibroblasts treated with cisplatin. We showed that NIH3T3 cells tolerated accumulation of both alpha globin and Bax under healthy growth conditions and that cell death progressed with faster kinetics in GFP-alpha globin and GFP-Bax transduced cells than in GFP control cells. We used the CellTiter-Glo Luminescent Cell Viability Assay instead of the well established TUNEL assay. TUNEL staining failed in trypsinized NIH3T3 cells.

In summary, there is strong evidence that the function of alpha globin is not restricted to oxygen transport in erythroid cells. Rather alpha globin seems to be involved in pro-apoptotic processes in a wide range of cells. These findings are reminiscent of observations made for another small protein, cytochrome c. Cytochrome c was initially believed to function exclusively as an electron carrier in the respiratory chain of mitochondria. Eight years ago, Liu et al found that cytochrome c was an essential component of the apoptosome (Liu et al., 1996). 


\subsection{GATA-2 promotes upregulation of alpha globin and apoptosis in FL5.12 cells}

\subsubsection{Expression and regulation of hematopoietic transcription factors in PCD}

After having shown the significance of alpha globin as a pro-apoptotic factor we searched for transcriptional or regulatory processes that drive alpha globin expression under apoptotic conditions. In erythroid cells the globin gene clusters are subject to several levels of regulation to ensure fine-tuned globin expression during defined periods of development, assuring correct balances of alpha and beta globin chains for hemoglobin assembly. This control depends on the complex interplay of chromatin structure, regulatory DNA sequences, and transcription factors (Cao and Moi, 2002). In this context, erythroid transcription factors have been intensively investigated and a model evolved suggesting a hierarchy of transcription factors according to the number of genes and circuits they regulate. GATA-1 and -2 are described to act on large numbers of erythroid genes at different stages of differentiation. They are therefore believed to act as master regulators. In contrast, other transcription factors such as EKLF, FOG-1 or Sp1 control a limited number of erythroid genes and often exhibit the function of coactivators for GATA transcription factors. The transcription factors that transactivate alpha and beta globin gene expression are partly overlapping (GATA-1, GATA-2, Sp-1, NF-E2) and partly unique to each globin gene. While transcription factors $\mathrm{CP}-2$, NF I, and NF- $\mathrm{KB}$ transcription factors are believed to predominantly act on alpha globin expression, EKLF and FOG-1, both cofactors of GATA-1, are mentioned in the context of beta globin transactivation. In terms of globin gene expression, it is hard to distinguish whether GATA-1 or GATA-2 exerts the regulatory mechanisms. In principle both GATA transcription factors can bind and transactivate globin genes. But since GATA-1 is more abundantly expressed in later erythroid cells it is usually presumed that GATA-1 is the protein acting at these sites. However, GATA-1 gene ablation studies in proerythroblasts and mice showed that GATA-2 could successfully substitute functions believed to be exclusive for GATA-1 (Weiss et al., 1997; Weiss and Orkin, 1995b).

In the current work, we focused on transcription factors, which were concomitantly regulated with alpha globin in apoptosis using Affymetrix gene chip arrays; i.e. our studies did not account for transcription-independent processes like protein stabilization or posttranslational modifications of transcription factors such as de-/phosphorylation (Towatari et al., 1995), dissociation from complexing inhibitory proteins (Brunet et al., 1999) or modulation of the redox state (Toledano and Leonard, 1991).

Analyzing the transcriptional response of IL-3-deprived and control FL5.12 cells, we found several upregulated erythroid transcription factors such as GATA-1, GATA-2, EKLF, FOG-1 and NF-E2, which could all theoretically promote alpha globin expression. These transcription factors can also regulate beta globin expression, which is probably why beta globin was found to be absent under normal growth conditions and exceeded the critical

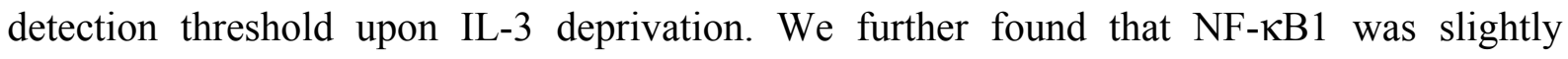
downregulated in apoptosis. NF- $\mathrm{KB} 1$ generally induces the expression of antiapoptotic genes, 
including Bcl- $\mathrm{x}_{\mathrm{L}}$, cIAP2, and c-FLIP and it has been described to inhibit the expression of alpha globin-like genes in early erythroid progenitors (Liu et al., 2003). I.e. downregulation of NF- $\kappa \mathrm{B} 1$ in death-committed FL5.12 cells possibly promotes apoptosis in two ways: by a decrease of pro-survival factors and concomitant upregulation of alpha globin.

We detected high level expression for GATA-2 in FL5.12 cells, whereas GATA-1 was expressed only at low level. This was in line with findings that GATA-2 is predominantly expressed in hematopoietic progenitor cells while GATA-1 is preferentially expressed in mature erythroid cells (Yamamoto et al., 1990; Ohneda and Yamamoto, 2002). Further, GATA-2 displayed the earliest and strongest transcriptional response towards cytokine deprivation; within one hour after IL-3 removal, GATA-2 was upregulated by a factor of two indicating an immediate early response. In contrast, upregulation of GATA-1, EKLF, FOG-1, and NF-E2 was delayed and first detected after 8 hours. The latter four transcription factors all contain GATA binding sites in their promotors, which suggests that their own transcription can be regulated by GATA factors (Anderson et al., 2000). In erythroid cells, a model has been proposed, in which GATA-2 binds to the GATA box in the promotor of GATA-1 and promotes GATA-1 expression in the early stage of erythroid differentiation (Orkin, 1995). We therefore suggest that GATA-2 may possibly trigger both direct activation of alpha globin transcription and indirect activation through the transcriptional activation of other alpha globin-specific transcription factors. In contrast to cytokine deprivation, treatment with cytotoxic compounds such as cisplatin or doxorubicin did not lead to upregulated GATA-2 levels in FL5.12 cells even though alpha globin was upregulated. We propose two alternative mechanisms: first, a transcription factor other than GATA-2 transactivates alpha globin in cisplatin- or DOXO-treated cells; second, stabilization and accumulation of alpha globin message is responsible for the 2- to 3-fold upregulated alpha globin levels.

Next we wanted to find out whether the upregulation of GATA transcription factors was unique to FL5.12 cells. We therefore examined the expression of GATA-1 and GATA-2 under apoptotic conditions that caused elevated alpha globin levels in NIH3T3, HeLa, and K562 cells. As seen in FL5.12 cells, increased GATA-2 transcript levels were also measured in NIH3T3 cells. In contrast, GATA-1 was not expressed in the non-hematopoietic NIH3T3 cell line. Concerning GATA-2, the two human cancer cell lines, HeLa and K562, exerted the opposite regulatory effects; GATA-2 levels decreased dramatically under death-inducing conditions. GATA-1 was not expressed in HeLa and was slightly downregulated in K562 cells. We therefore suggest that enhanced transcription of alpha globin in HeLa and K562 are regulated independently of GATA transcription factors.

\subsubsection{Effects of overexpression and silencing of GATA-2 on apoptosis and alpha globin expression}

To elucidate whether GATA-2 acts as a transcription factor of alpha globin, we overexpressed GATA-2 in FL5.12 and NIH3T3 cells. In both cell lines, ectopically expressed GATA-2 was localized to the nuclei, the correct destination of a transcription factor. NIH3T3 cells overexpressing GATA-2 demonstrated a dramatic stress phenotype with reduced proliferation rate especially at low cell densities. This phenotype was transient, however, and cells reverted 
to a normal morphology within two weeks post transduction. A second round of sorting was well tolerated without phenotypical changes. Despite high level expression of GFP-GATA-2 in NIH3T3 cells, we failed to detect increased alpha globin levels. We suggest three possible explanations for the missing transactivation of alpha globin: first, NIH3T3 cells adjust to increased GATA-2 expression levels and establish a negative feedback mechanism that counteracts upregulated alpha globin levels; second, erythroid genes in fibroblasts are compacted in inactive chromatin and are therefore not accessible for transcription factors; or third, GATA-2 alone is not able or not sufficient to activate alpha globin expression. According to Dubart et al, GATA-1 overexpression in NIH3T3 cells had a similar negative effect on the proliferation as GATA-2 in our experiments. The substantial impairment of proliferation was transient and was especially seen at low cell densities. It was further observed that overexpressed GATA-1 failed to transactivate its erythroid-specific target genes (Dubart et al., 1996).

Overexpression and enrichment of GATA-2 in FL5.12 cells led to immediate cell death. The cells displayed characteristics of cells undergoing apoptosis such as membrane blebbing and loss of plasma membrane integrity. To date, GATA transcription factors are primarily believed to regulate processes supporting survival, differentiation, and proliferation of cells (Ohneda and Yamamoto, 2002; Ting et al., 1996). During erythropoiesis, committed erythroid precursors arrest at the proerythroblast stage of development and undergo apoptosis in the absence of GATA-1 (Weiss et al., 1997). In the erythropoietin-dependent cell line, AS-E2, erythropoietin triggers proliferation and survival by maintaining high GATA-2 and Bcl- $\mathrm{x}_{\mathrm{L}}$ expression (Tsushima et al., 1997). Induction of apoptosis by death receptor ligands results in caspase-mediated degradation of GATA factors (De Maria et al., 1999). And finally, in the FL5.12 related cell line, BaF3, GATA factors are believed to transduce the survival signal of IL-3 (Yu et al., 2002). These findings are reminiscent of our observations in HeLa and K562 cells. GATA-1 and GATA-2 levels were strongly downregulated in HeLa and K562 cells undergoing apoptosis, suggesting that GATA factors play a role in signaling proliferation and survival and that they are downregulated in death-committed cells.

Conversely, there is evidence that overexpression of GATA transcription factors exert negative effects on proliferation and survival. GATA-2 transcript levels were upregulated by a factor of 3.5 in the human epidermoid carcinoma cell line A431 after treatment with the tyrosine kinase inhibitor PTK166 for $24 \mathrm{~h}$. A431 cells overexpress epidermal growth factor receptors (EGF-R) and were found to undergo apoptosis upon EGF-R inhibition by PTK166 (Adrian Brüngger, Novartis Basel, personal communication). Persons and colleagues found that enforced expression of GATA-2 in bone marrow cells blocked both their amplification and differentiation. However, cells remained viable and were negative in apoptosis assays (Persons et al., 1999). Induction of estrogen and tamoxifen-inducible forms of GATA-2 but not GATA-1 in IL-3 dependent FDC-P1 cells blocked factor-dependent self-renewal. GATA-2 activation was further accompanied by differentiation down the monocytic and granulocytic lineage (Heyworth et al., 1999). Similarly, Ikonomi and colleagues observed that overexpression of GATA-2 in K562 cells had a negative effect on cell proliferation (Ikonomi et al., 2000). This phenotype, however, was not further investigated. Iwasaki et al reported 
that enforced expression of GATA-1 was accompanied by apoptosis in pro B and myelomonocytic cells. The death-inducing effect of GATA-1 was not even abrogated in cells ubiquitously expressing human Bcl-2 (Iwasaki et al., 2003). This prompts us to test whether Bcl-2 or Bcl- $\mathrm{x}_{\mathrm{L}}$ can prevent GATA-2-induced apoptosis in FL5.12 cells in future experiments.

In parallel to viability tests, we assessed the expression of alpha globin in FL5.12 cells transduced with GATA-2. In contrast to NIH3T3 cells we measured significantly upregulated alpha globin levels in GATA-2 expressing cells. FLAG- and GFP-GATA-2 transduced FL5.12 cells displayed 6- and 3-fold elevated endogenous alpha globin levels, respectively, as compared to GFP expressing cells. Upregulation of alpha globin was probably not sufficient to induce the apoptotic phenotype, since overexpression of alpha globin in the presence of IL-3 alone was tolerated without induction of cell death. Further evidence that GATA-2 triggers upregulation of endogenous alpha globin in FL5.12 cells in the absence of IL-3 derived from experiments using antisense GATA-2. Antisense GATA-2 did not reduce basal expression of alpha globin under normal growth conditions. In the absence of cytokine, however, induction of alpha globin protein was diminished by a factor of 2 as compared to GFP expressing cells. Concomitantly, both the progression of cell death and the caspase-3 activity were significantly reduced by $40 \%$ and $58 \%$, respectively. This suggests that endogenous GATA-2 is involved in the upregulation of alpha globin after IL-3 removal but not in the basal expression under normal growth conditions.

As mentioned above, GATA transcription factors have been reported to exert cross-regulatory transactivation. To clarify whether ectopic GATA-2 transactivated GATA-1 and whether the apoptotic phenotype was at least partly mediated through co-activated GATA-1, we assessed the expression of GATA-1 in both GATA-2-overexpressing cells and cells expressing the antisense GATA-2. We showed that endogenous GATA-1 levels were entirely unaffected in both experimental set ups suggesting that GATA-1 is not involved in GATA-2-induced apoptosis signaling. However, we cannot conclude, whether this model reflects the endogenous situation in FL5.12 cells. The mechanisms how GATA-2 induces cell death should be elucidated in future studies. Especially gene expression profiling experiments using FL5.12 cells expressing GATA-2 under a Tet-inducible/repressible promotor could shed light on other factors which are under control of GATA-2.

In summary, we have shown that GATA-2 triggers apoptosis and induces transcription of alpha globin in the hematopoietic cell line FL5.12. However, GATA-2 probably acts as a transcription factor also for pro-apoptotic factors other than alpha globin, since FL5.12 cells overexpressing GATA-2 died by apoptosis even in the presence of IL-3, whereas alpha globin overexpression accelerated apoptosis only in the absence of the cytokine. Furthermore, it appears that also mechanisms or transcription factors other than the GATA factors exist to control the regulation of alpha globin in apoptosis, especially in HeLa and K562 cells. 
The concluding model summarizes our findings on alpha globin and GATA-2 in apoptotic cell death in FL5.12 cells:

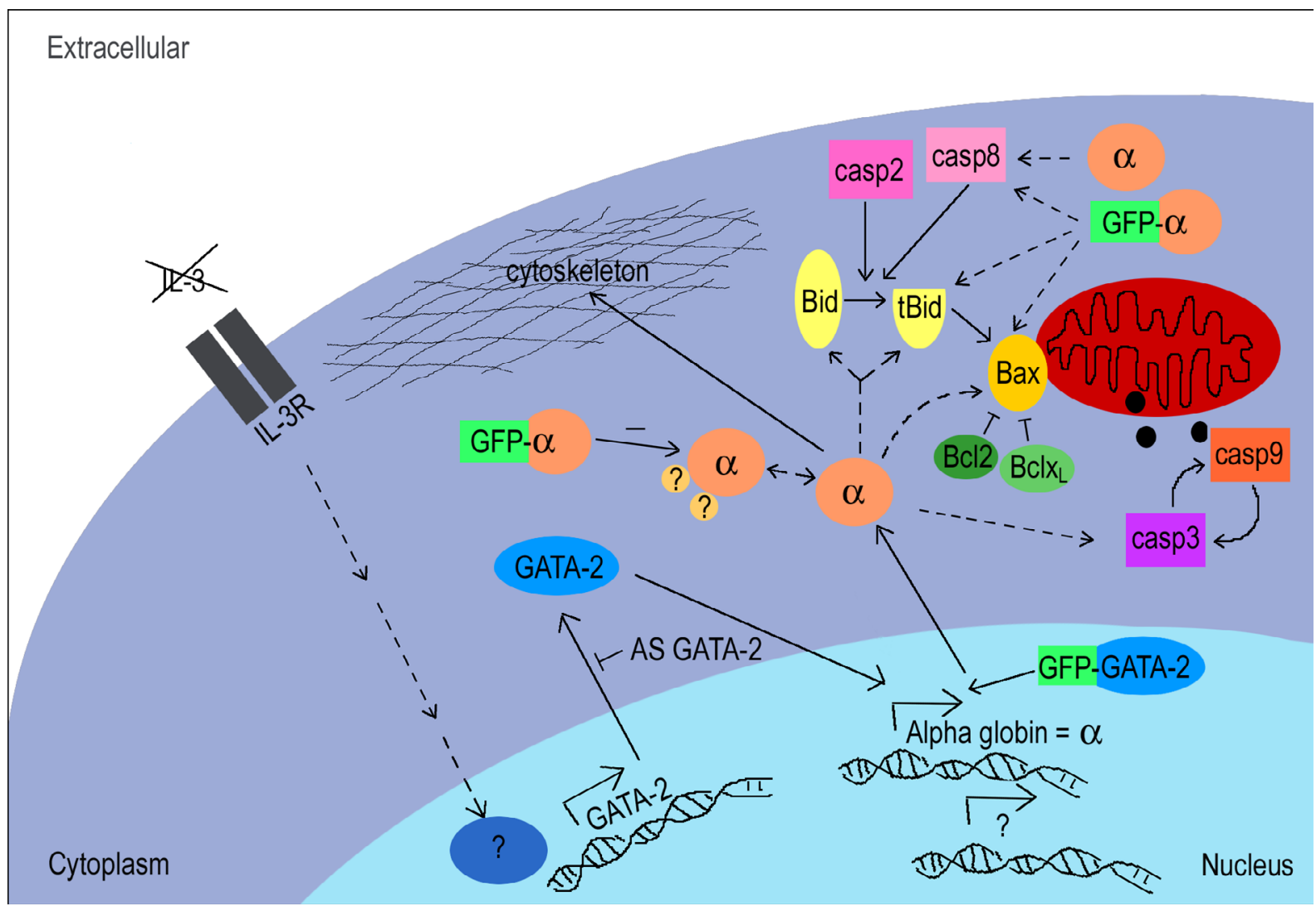

GATA-2 and alpha globin in IL-3 deprivation induced apoptosis. IL-3 deprivation leads to upregulation of GATA-2, which in turn transactivates alpha globin $(\alpha)$ and probably other, unknown pro-apoptotic factors. Alpha globin accelerates the mitochondrial pathway possibly by interfering with the processing of caspases, or Bid to truncated Bid (tBid), or with the translocation of Bax. Alpha globin may be posttranslationally modified. Small amounts of unmodified alpha globin translocates to the cytoskeleton. Ectopic GFP-alpha globin (GFP- $\alpha$ ) accelerates apoptosis and reduces synthesis of endogenous alpha globin. AS GATA-2 diminishes the upregulation of GATA-2 and alpha globin and decelerates the apoptotic process; GFP-GATA-2 induces upregulation of alpha globin and cell death. Anti-apoptotic Bcl-2 and Bcl- $x_{\mathrm{L}}$ prevent the pro-apoptotic effect of alpha globin. Unknown signaling paths are depicted by dashed lines. Black circles show cytochrome c. 



\section{$5 \quad$ Materials and Methods}

\subsection{Cell lines, treatments}

Murine fibroblast cell line, NIH3T3, was obtained from ATCC (Rockville) and grown in MEM Alpha medium supplemented with 15\% heat-inactivated fetal bovine serum (HyClone), $1 \%$ sodium pyruvate, (Invitrogen), 1\% non-essential amino acids, (Invitrogen), $2 \mathrm{mM}$ L-glutamine (Invitrogen), and $10 \mathrm{mg} / 1$ gentamicin (Invitrogen). NIH3T3 cells were 50\% confluent when treatment with cisplatin $(20 \mu \mathrm{M})$ and staurosporine (STS) $(1 \mu \mathrm{M})$ was started. Controls were left untreated or grown in the presence of DMSO.

293T-derived Phoenix Eco packaging cell line and HeLa cells were grown in Dulbecco's modified Eagle's medium (DMEM) supplemented with $10 \%$ fetal calf serum (FCS) (BioConcept) and 1\% Penicillin-Streptomycin-Fungizone $(250 \mathrm{ng} / \mathrm{ml})$ (BioConcept). Treatment with cisplatin $(20 \mu \mathrm{M})$ and STS $(0.5 \mu \mathrm{M})$ was started when HeLa cells were $50 \%$ confluent. Cells growing in normal medium or treated with DMSO (1:500 dilution) served as controls.

Human K562 cells were cultured in RPMI 1640 medium with 10\% FCS (BioConcept). Treatment of K562 cells with hemin (Sigma) (Taketani et al., 1998), TRAIL (Sigma) $(250 \mathrm{ng} / \mathrm{ml}$ in phosphate buffered saline (PBS)), STI571, imatinib mesylate (500 $\mathrm{nM}$ in DMSO) (Jürgen Mestan, Novartis), or DMSO as control was started at a cell density of $5 \times 10^{5}$ cells $/ \mathrm{ml}$.

Mouse erythroleukemic J2E cells were kindly provided by Thomas Bittorf (University Rostock). Cells were cultured in DMEM supplemented with 10\% HyClone II FCS.

Murine FL5.12neo cells stably transfected with a vector for G418 resistance were kindly provided by Dr. Korsmeyer. These cells were used in all experiments and are referred to as FL5.12 cells. Cells were grown in RPMI 1640 medium with 10\% heat-inactivated FCS (BioConcept), 1\% Penicillin-Streptomycin-Fungizone (BioConcept), $100 \mu \mathrm{M} \beta$-mercaptoethanol (Invitrogen), G418 sulfate $(200 \mathrm{mg} / 1)$, and mouse, recombinant interleukin 3 (IL-3) $(1 \mathrm{ng} / \mathrm{ml})$ (Sigma). For treatment FL5.12 cells were seeded at a density of $2 \times 10^{5}$ cells $/ \mathrm{ml}$ and subjected to cisplatin (20 $\mu \mathrm{M}$ in $\left.\mathrm{H}_{2} \mathrm{O}\right)$, STS (20 nM in DMSO), doxorubicin (DOXO) (40 nM in $\mathrm{H}_{2} \mathrm{O}$ ), or tumor necrosis factor alpha (TNF alpha) $(2 \mathrm{ng} / \mathrm{ml}$ in PBS containing $1 \%$ bovine serum albumin (BSA)) and cycloheximide (CHX) $(1 \mu \mathrm{g} / \mathrm{ml}$ in DMSO). All compounds were purchased from Sigma Aldrich, St. Louis, Missouri, USA. For IL-3 deprivation experiments cells were washed three times with medium lacking IL-3 before they were cultured in IL-3free medium. Control cells growing in medium containing IL-3 were subjected to the same washing. Pan caspase inhibitor zVAD-fmk (Sigma) (50 $\mu \mathrm{M}$ in DMSO) were used to assess caspase inhibition in IL-3-deprived FL5.12 cells.

All cell lines were cultivated at $37^{\circ} \mathrm{C}$ in a $5 \% \mathrm{CO}_{2}$ humidified atmosphere. 


\subsection{Transient transfection of HeLa cells}

HeLa cells were grown to $40-60 \%$ confluency in a T75 cell culture flask and were then transfected with GFP, GFP-alpha globin, GFP-beta globin major chain or GFP-AHSP using FuGene 6 (Roche). Briefly, $20 \mu \mathrm{l} \mathrm{FuGene} \mathrm{were} \mathrm{mixed} \mathrm{with} 300 \mu \mathrm{l}$ OptiMEM (Gibco) and incubated for $5 \mathrm{~min}$ at room temperature. The mixture was then dropwise added to $5 \mu \mathrm{g}$ plasmid DNA and incubated for additional $15 \mathrm{~min}$. The final mix was pipetted to the cells, swirled, and incubated in a $37^{\circ} \mathrm{C}$ incubator for $48 \mathrm{~h}$.

\subsection{Gateway expression cloning and retroviral transduction}

Genes of interest were synthesized with PCR. cDNA, derived from total RNA of FL5.12 or HeLa cells, served as a template. The template for mouse AHSP was a gift of Mitchell Weiss. Oligonucleotide primers contained attB sites at their respective 5 ' ends (forward primer: GGGG ACA AGT TTG TAC AAA AAA GCA GGC TCC, reverse primer: GGGGAC CAC TTT GTA CAA GAA AGC TGG GTA) to render cDNA templates compatible with the GATEWAY $^{\mathrm{TM}}$ cloning technology (Life Technologies). Primers for human $b c l-x_{L}$ (5`ATGTCTCAGAGCAACCGGGAGCTGG， 5`CTATTTCCGACTGAAGAGTGAGCCCAGCAG), human bax (5 ATGGACGGGTCCGGGGAGCAGCCCAGAGGC, 5 CTAGCCCATCTTCTTCCAGATGGTGAGCGAGGC), mouse alpha globin (5`ATGGTGCTCTCTGGGGAAGAC, 5'CTAACGGTACTTGGAGGTCAGCAC), antisense mouse alpha globin (5' TAAACGGTACTTGGAGGTCAGCACGGTG, 5 'ATGGTGCTCTCTGGGGAAGACAAAAGCAAC), mouse beta globin major chain (5`ATGGTGCACCTGACTGATGCTGAG, 5`TTAGTGGTACTTGTGAGCCAGGGC) mouse GATA-2 (5`ATGGAGGTGGCGCCTGAGCAGCCTCGC, 5`GCCCATGGCAGTCACCATGCTGG), antisense mouse GATA-2 (5` TAACTAGCCCATGGCAGTCACCATGCTG, 5`ATGGAGGTGGCGCCTGAGCAGC), mouse AHSP (5`ATGGCCCTTTTCAGAGCAATAAGGATC, 5`TTATGAGGAGGGCAGTGTATTGCTTGG) were used.

In the $\mathrm{BP}^{\mathrm{TM}}$ clonase reaction PCR products were first cloned into the $\mathrm{pDONR}^{\mathrm{TM}}$ (Life Technologies) vector and were then verified by sequencing. In the second $\mathrm{LR}^{\mathrm{TM}}$ clonase reaction cDNA inserts were transferred into retrovirus-derived expression vectors called destination vectors. Two destination vectors, CRU5-FLAGgw-IRES-GFP (Figure 1, upper panel) and CRU5-N-term-GFPgw (Figure 1, lower panel), were used which were both generated by Dr. Benoit Pierrat (Novartis Basel). Both vectors contained a beta-lactamase gene rendering ampicillin resistance $(\mathrm{AmpR})$, a constitutive cytomegalo virus (CMV) promotor, sequences from long terminal repeats ( $\left.5^{`} \mathrm{RU}, 3^{`} \mathrm{LTR}\right)$ rendering the plasmid a retroviral vector, Gateway recombination cassettes (attR), and enhanced GFP (eGFP). For simplicity reasons eGFP is referred to as GFP. Using the CRU5-FLAGgw-IRES-GFP vector the genes of interest, AHSP and GATA-2, were expressed as N-terminal FLAG-tagged proteins with an internal ribosomal entry site (IRES) for GFP. With the CRU5-N-termGFPgw vector all other genes of interest used in the current work were expressed as Nterminal GFP fusion proteins.

Once constructs had been verified by restriction digest they were transfected using the calcium-phosphate method into Phoenix packaging cells expressing an ecotropic envelope 
protein. Virus containing supernatants were then used to transduce FL5.12 or NIH3T3 cells (Swift et al., 1999). Efficiency of transduction was followed by monitoring GFP expression levels in a FACS Calibur instrument (Becton Dickinson). One to two rounds of cell sorting were used to enrich GFP-expressing cells.
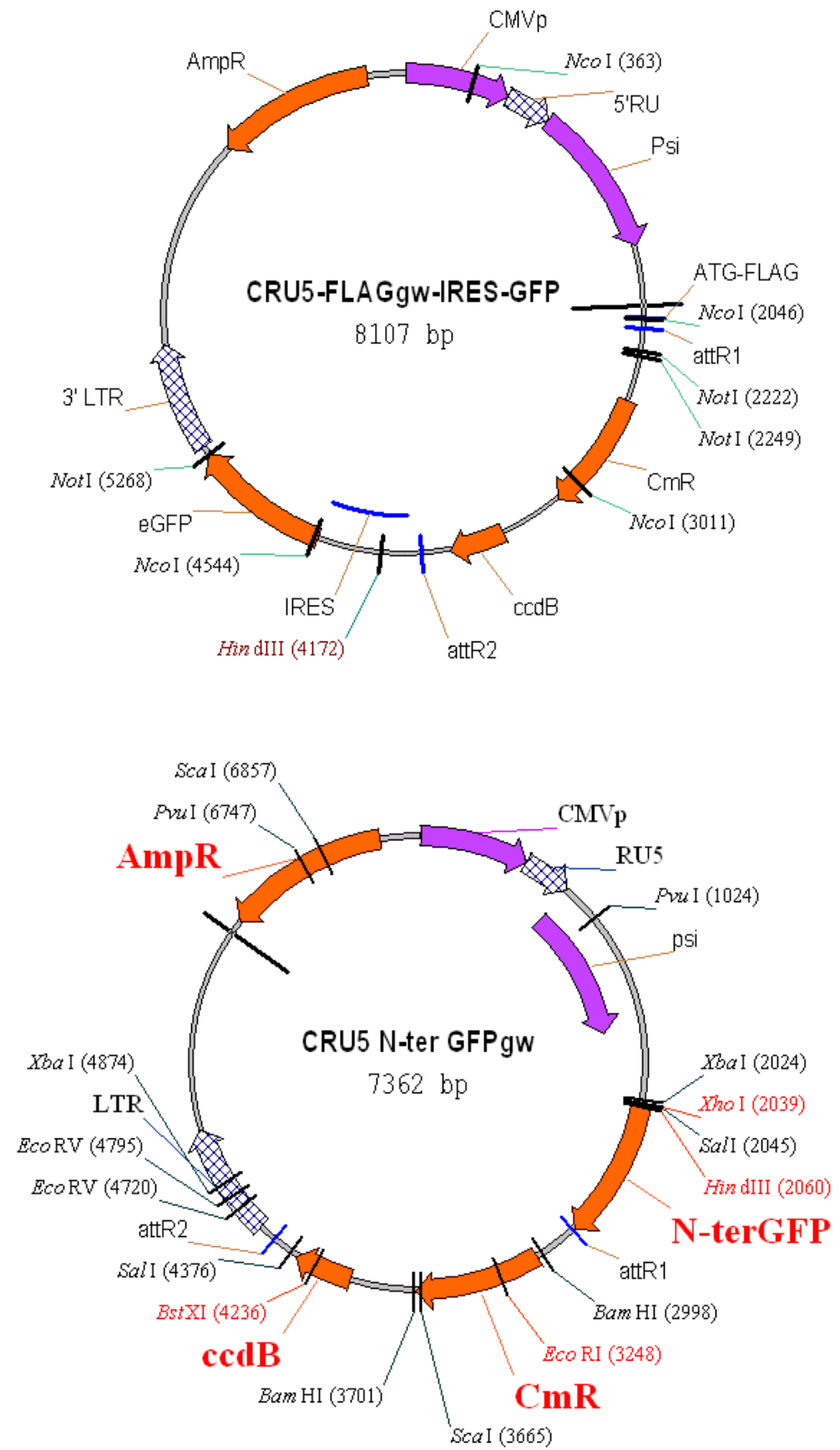

Figure 1: Plasmid constructs of retrovirus-derived, Gatewaycompatible destination vectors, CRU5-FLAGgw-IRES-GFP (upper panel) and CRU5-N-term-GFPgw (lower panel) (redrawn from Vector NTI). 


\subsection{Proliferation test}

FL5.12 cells ectopically expressing GFP, GFP-Bax or GFP-alpha globin as well as untransduced FL5.12 cells were seeded at a density of $2 \times 10^{4}$ cells $/ \mathrm{ml}$. Cell viability and density was assessed by trypan blue staining and counting after 14, 20, and $36 \mathrm{~h}$ using a Neubauer hemocytometer. For each cell line three separate T25 cell culture flasks were grown and independently analyzed.

\subsection{TUNEL assay}

To study DNA fragmentation, $1 \times 10^{6}$ cells were subjected to terminal deoxynucleotidyl transferase dUTP nick end labeling (TUNEL) staining using the In Situ Cell Death detection kit (Roche). Protocol was performed as described by the manufacturer: Cells were washed with PBS, fixed with 2\% paraformaldehyde (PFA) in PBS, $\mathrm{pH} 7.4$ for $1 \mathrm{~h}$ at room temperature, and then permeabilized with $0.1 \%$ Triton-X 100 in $0.1 \%$ sodium citrate for 2 min on ice. The TUNEL reaction with terminal deoxynucleotidyl transferase (TdT) and TMR red labeled nucleotides was performed for $1 \mathrm{~h}$ at $37^{\circ} \mathrm{C}$ in humidified atmosphere and stopped by washing with PBS. TUNEL-positive cells were monitored using a FACS Calibur (Becton Dickinson) (FL2-H, 550/30nm).

\subsection{Caspase Profiling}

The ApoAlert ${ }^{\mathrm{TM}}$ Caspase Assay Plate (Becton Dickinson) was used to assay the apoptotic activity of caspase-2, $-3,-8$, and -9 in a 96-well format. Briefly, transduced FL5.12 cells were allowed to grow with and without IL-3 for $12 \mathrm{~h}$. 2 × $10^{5}$ cells per well were analyzed. Single measurements were performed for cells growing with IL-3 and cells growing without IL-3 plus the specific caspase inhibitor. For cells growing in medium lacking IL-3 duplicates were analyzed. Cells were lyzed on ice for $10 \mathrm{~min}$ with lysis buffer provided with the ApoAlert ${ }^{\mathrm{TM}}$ kit, transferred to the caspase assay plate coated with immobilized, fluorogen-labeled caspase substrates and incubated at $37^{\circ} \mathrm{C}$ for $1.5 \mathrm{~h}$. Fluorescence intensity was monitored after 0 and $1.5 \mathrm{~h}$ using the Analyst ${ }^{\circledR}$ GT Reader (405 nm excitation, $460 \mathrm{~nm}$ emission) (Bucher Biotec). Caspase profiling was repeated once and each caspase status was further confirmed by Western blotting.

\subsection{Caspase-3 Assay}

FL5.12 cells ectopically expressing GFP, antisense GATA-2 or antisense alpha globin were grown in the presence or absence of IL-3 for 14 hours. Caspase- 3 activity was measured using the Caspase-3 Colorimetric Assay kit (BioVision). Briefly, $1.5 \times 10^{6}$ cells were lyzed in $70 \mu 1$ cell lysis buffer. $10 \mu 1$ lysates were mixed with $40 \mu 1$ cell lysis, $50 \mu 12 x$ reaction buffer, and 5 $\mu 14 \mathrm{mM}$ DEVD-pNA (200 $\mu \mathrm{M}$ final concentration) and incubated at $37^{\circ} \mathrm{C}$ for $1.5 \mathrm{~h}$ in a 96well optical plate (Nalge Nunc). Triplicates were read at $405 \mathrm{~nm}$ using a Analyst ${ }^{\circledR}$ GT Reader.

\subsection{CellTiter-Glo ${ }^{\mathrm{TM}}$ Luminescent Cell Viability Assay}

NIH3T3 cells ectopically expressing GFP, GFP-alpha globin or GFP-Bax were seeded in a 96-well optical plate at a density of 4000 cells per well. 24 hours after seeding medium was replaced with fresh medium (control) or medium containing $20 \mu \mathrm{M}$ cisplatin. Viability of 
transduced NIH3T3 cells was assessed 20 hours after incubation using CellTiter-Glo ${ }^{\mathrm{TM}}$ Luminescent Cell Viability Assay (Promega). Briefly, CellTiter-Glo ${ }^{\mathrm{TM}}$ buffer and substrate as well as the plate containing the cells were equilibrated at room temperature for $30 \mathrm{~min}$. CellTiter-Glo ${ }^{\mathrm{TM}}$ reagent was prepared by dissolving CellTiter-Glo ${ }^{\mathrm{TM}}$ substrate (luciferin) in the buffer. $100 \mu 1$ of the complete reagent were added to each well. The plate was mixed and then incubated for 2 and $10 \mathrm{~min}$, respectively, to stabilize the luminescent signal. Luminescence was measured using a Tecan Ultra Evolution reader (Tecan). Cell culture medium alone served as fluorescent background control.

\subsection{Total RNA isolation}

Total RNAs were purified from FL5.12, NIH3T3, HeLa, and K562 cells using an RNeasy spin column system according to the manufacturer's instructions (Qiagen). Briefly, cells were pelleted, lyzed with $\mathrm{RLT}^{\mathrm{TM}}$ lysis buffer and subsequently homogenized using QIAshredder ${ }^{\mathrm{TM}}$ spin columns (Qiagen). Isolation of total RNA was performed with a silica-gel-based membrane technology (RNeasy Mini Kit, Qiagen). RNase-free DNase (Qiagen) kit was used to avoid DNA contamination in the isolated RNA. RNA quality assessing $18 \mathrm{~S}$ and $28 \mathrm{~S}$ ribosomal bands was analyzed in an Agilent 2100 LabChip Bioanalyzer using RNA 6000 Nano Chips ${ }^{\mathrm{TM}}$ (Agilent Technologies). RNA concentration and purity (A260/A280nm) were measured using the BioSpec-mini ${ }^{\mathrm{TM}}$ spectrophotomer (Shimadzu).

\subsection{Affymetrix gene expression arrays}

Total RNA was converted into double-stranded cDNA using Superscript II reverse transcriptase and an oligo(dT) primer with a T7 RNA polymerase promoter site at the 5 ' end (Invitrogen). $5 \mu \mathrm{g}$ cDNA were used in an in vitro transcription in the presence of biotinylated ribonucleotides and T7 RNA polymerase to produce labeled cRNA (Enzo diagnostics of Farmingdale). $15 \mu \mathrm{g}$ cRNA were randomly fragmented by incubating in $40 \mathrm{mM}$ Tris-acetate, $\mathrm{pH} 8.1,100 \mathrm{mM}$ potassium acetate, and $30 \mathrm{mM}$ magnesium acetate at $94^{\circ} \mathrm{C}$ for $35 \mathrm{~min}$. Affymetrix Murine Genome U74v2 gene chips (Affymetrix, San Jose, CA) were hybridized, washed, and stained according to the protocol (Mahadevappa and Warrington, 1999). Fluorescence intensities for the individual oligonucleotide probes on the arrays were determined with a GeneChip system confocal scanner (Hewlett Packard) and analyzed using the Demon Novartis proprietary software program. All chips were prepared and analyzed in duplicates.

\subsection{Quantitative polymerase chain reaction, RT QPCR}

Total RNA was reverse transcribed using Omniscript ${ }^{\mathrm{TM}}$ RT kit (Qiagen) and rRNasin ${ }^{\circledR}$ RNase inhibitor (Promega). cDNAs were subjected to 40 rounds of amplification in the presence of TaqMan ${ }^{\circledR}$ Master Mix (Applied Biosystems) using the ABI Prism 7700 sequence detection system as described by the manufacturer (Applied Biosystems). Sequence-specific primers and probes labeled with a reporter, 6-carboxyfluorescein (6-FAM), and a quencher, 6-carboxytetramethylrhodamine (6-TAMRA), were designed with the primer express software (Applied Biosystems) or ordered as ready to use primer and probe mixes (Assay on Demand, Applied Biosystems). The following specific primer/probe sets were used: 
mouse alpha globin forward primer: 5 GCCTTGTCTGCTCTGAGCG, reverse primer: 5' CTTGAAGTTGACGGGATCCAC, probe: 5 CCTGCATGCCCACAAGCTGCG; human alpha globin forward primer: 5 TCCCCACCACCAAGACCTAC, reverse primer: 5 CCTTAACCTGGGCAGAGCC, probe: 5'CCCGCACTTCGACCTGAGCCA; mouse AHSP forward primer: 5 TTGTGGTTCATGACTGGGTGA, reverse primer: 5'CCTGTCTTGCTCCTCCTGCT, probe: 5`ACCAACTATTACAAGAAGCTCGTGCATG; mouse heme oxygenase 1 forward primer: 5'CTCACTGGCAGGAAATCATCC, reverse primer: 5'ACCTCGTGGAGACGCTTTACA, probe: 5' CCTGGAGGAGGAGATTGAGCGCAAC; mouse beta globin: Assay-on-Demand Gene Expression product, Mm00731743_mH; human beta globin: Assay-on-Demand Expression product, Hs00747223_g1; mouse GATA-2: Assay-on-Demand Gene Expression product, Mm00492300_m1; human GATA-2: Assay-on-Demand Gene Expression product, Hs00231119_m1; mouse GATA-1: Assay-on-Demand Gene Expression product, Mm00484678_m1; human GATA-1: Assay-on-Demand Gene Expression product, Hs00231112_m1; eukaryotic ribosomal RNA (18S rRNA): Genomic Assay, 4310892E.

10 ng cDNA were used as template for RT QPCR. Specific amplification was detected as an increased fluorescence signal of 6-FAM during the amplification cycle. The relative quantity of specifically amplified cDNAs were calculated using the comparative-threshold cycle method with interspecific $18 \mathrm{~S}$ ribosomal RNA as endogenous reference according to the manufacturer's instructions (Applied Biosystems, User Bulletin \#2, ABI PRISM 7700). No template controls (NTC) and no reverse transcriptase controls (noRT) were always performed in parallel to exclude transcript unspecific amplification.

\subsection{PCR on genomic DNA}

$2 \times 10^{5}$ FL5.12 cells transduced with FLAG-alpha globin IRES GFP were washed twice with PBS and subsequently resuspended and lyzed for $1 \mathrm{~h}$ at $37^{\circ} \mathrm{C}$ in $50 \mu 1 \mathrm{x}$ Taq PCR reaction buffer (Roche) containing $0.05 \%$ SDS and $50 \mu \mathrm{g} / \mathrm{ml}$ proteinase $\mathrm{K}$. Proteinase $\mathrm{K}$ was then heat-inactivated for $20 \mathrm{~min}$ at $85^{\circ} \mathrm{C}$. Cell lysates were diluted 1:10 in Taq polymerase buffer. $1 \mu \mathrm{l}$ of the dilution served as template in the following PCR. The PCR reaction mix contained 10\% DMSO (Sigma), 10\% Taq PCR reaction buffer, $200 \mu \mathrm{M}$ desoxynucleotidyltriphosphates

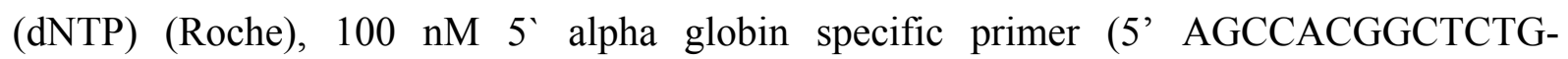
CCCAGG), $100 \mathrm{nM}$ 3' alpha globin specific primer (5' GAGGTCAGCACGGTGCTCACAG), and 5 units Taq polymerase (Roche). The reaction mix was denatured 3 min at $95^{\circ} \mathrm{C}$, followed by 30 PCR cycles: $30 \mathrm{sec}$ at $95^{\circ} \mathrm{C}$ denaturation, $40 \mathrm{sec}$ at $57^{\circ} \mathrm{C}$ annealing, and $30 \mathrm{sec}$ at $72^{\circ} \mathrm{C}$ elongation. PCR products were analyzed on a $1 \%$ agarose gel using DNA Molecular marker VI to assess product size.

\subsection{Western blotting}

For preparation of whole cell lysates cells were washed once with ice-cold PBS and then lyzed in ice-cold NET buffer containing $0.05 \mathrm{M}$ Tris-HCl, $50 \mathrm{mM} \mathrm{NaCl}, 5 \mathrm{mM}$ EDTA, and $1 \%$ Nonidet P-40 both supplemented with Complete protease inhibitor cocktail (Roche Diagnostics). To obtain soluble proteins cells were centrifuged at $14000 \mathrm{x} g$ to remove cellular debris. 
For fractionation of FL5.12 cells into cytosolic, membrane, nuclear, and cytoskeletal fraction the ProteoExtract ${ }^{\mathrm{TM}}$ Subcellular Proteome Extraction kit (Calbiochem) was used to analyze alpha globin localization. The protocol was followed according to the instructions of the manufacturer.

Separation of cytosolic and membrane proteins was done to evaluate localization of Bax, Bid, and cytochrome c. $2 \times 10^{6}$ FL5.12 cells were washed in PBS and resuspended in $200 \mu 1$ STE buffer containing $0.25 \mathrm{M}$ sucrose, $20 \mathrm{mM}$ Tris-HCl, $\mathrm{pH}$ 8, and $0.1 \mathrm{mM}$ EDTA supplemented with protease inhibitor cocktail. Cells were homogenized with a pellet pestle (Kontes Glass Company, NJ, USA) and a 30G1/2 Microlance needle (Becton Dickinson). Cell debris, intact cells and nuclei were removed by centrifugation at $1000 \mathrm{x} g$ for $5 \mathrm{~min}$ at $4^{\circ} \mathrm{C}$. The supernatants were centrifuged at $80000 \times \mathrm{g}$ for $1 \mathrm{~h}$ in a Beckman Airfuge to pellet the mitochondrial and microsomal fraction. Supernatants were precipitated with $14 \%$ trichloroacetic acid on ice for $30 \mathrm{~min}$, centrifuged and washed twice with cold acetone. All pellets were dissolved in $30 \mu \mathrm{l}$ Laemmli sample buffer, boiled for $3 \mathrm{~min}$ and $20 \mu \mathrm{l}$ from each fraction were loaded in $12 \%$ sodiumdodecylsulfate polyacrylamide (SDS PAGE) gels.

To assess GATA-2 localization cytosolic and nuclear fractions were prepared as follows: $1.5 \times 10^{6}$ FL5.12 cells were washed in PBS and resuspended in $200 \mu$ STE buffer (as described above). Cells were homogenized with a pellet pestle (Kontes Glass Company, NJ, USA) and a 30G1/2 Microlance needle (Becton Dickinson). Nuclei were removed by centrifugation at $610 \mathrm{xg}$ for $5 \mathrm{~min}$ at $4^{\circ} \mathrm{C}$. The nuclear pellets were resuspended with ice-cold Extraction buffer III and treated with Benzoenase ( $\geq 375 \mathrm{U}$ ) for $10 \mathrm{~min}$ on ice. Both reagents derived from the ProteoExtract ${ }^{\mathrm{TM}}$ Subcellular Proteome Extraction kit (Calbiochem). The supernatants contained cytosolic proteins. Cytosolic and nuclear proteins were taken up in Laemmli sample buffer, and boiled for $3 \mathrm{~min}$.

For all Western blots either $12 \%$ SDS PAGE or NuPage ${ }^{\circledR} 4-12 \%$ Bis-Tris gels (Invitrogen) were used. Gels were blotted onto Protran ${ }^{\circledR}$ nitrocellulose transfer membrane (Schleicher \& Schuell) using a Trans-Blot semidry blotting apparatus (BioRad). Membranes were blocked with 5\% milk powder (Rapilait, Migros, Switzerland), 1\% Tween 20 (Fluka) in TBS (TRISbuffered saline), and probed with anti-Actin polyclonal 1:1000 (Santa Cruz I-19), anti-Bax polyclonal 1:3000 (Santa Cruz N-20), anti-Green fluorescent protein (GFP) monoclonal 1:1000 (Roche), anti-Calnexin polyclonal 1:500 (Santa Cruz C-20), anti-cytochrome c monoclonal 1:500 (BD Pharmingen), anti-hemoglobin polyclonal 1:1000 (ICN Cappel), antiBid polyclonal 1:1000 (Cell Signaling), anti-caspase-2 monoclonal 1:500 (Alexis Biochemicals 11B4), anti-cleaved caspase-3 (Asp175) polyclonal 1:1000 (Cell Signaling), anti-caspase-7 polyclonal 1:100 (Santa Cruz K-20), anti-caspase-8 monoclonal 1:500 (Alexis Biochemicals 1G12), anti-caspase-9 monoclonal 1:500 (Stressgen Biotechnologies), and antiAHSP monoclonal 1:3000 (friendly gift from Mitchell Weiss, Children`s Hospital Philadelphia) antibodies. Secondary antibodies (BioRad) were HRP-conjugated goat antimouse IgG 1:5000, goat anti-rabbit IgG 1:5000, goat anti-rat IgG 1:5000, and rabbit anti-goat IgG 1:1000. All antibodies were diluted in TBS $+1 \%$ Tween 20 or blocking buffer. The proteins were visualized using Lumi-Light ${ }^{\text {PLUS }}$ Western Blotting Substrate (Roche) or ECL 
Western Blotting Detection Reagents (Amersham). Intensities of the signals were evaluated using a BioRad Imager and the Quantity One software program (BioRad). Blots were stripped for reuse using Re-Blot Western Blot Recycling (Chemicon) for $10 \mathrm{~min}$ at room temperature.

\subsection{Immunostaining, DAPI staining and fluorescence microscopy}

FL5.12 cells transduced with GFP-alpha globin, GFP-Bax, GFP or untransduced cells were grown with or without IL-3 for $12 \mathrm{~h}$. Cells were washed once with PBS, resuspended in $300 \mu \mathrm{l}$ PBS, spun on object trays with a Cytospin (Shandon Southern), and fixed with 4\% PFA for $15 \mathrm{~min}$. Thereafter the cells were permeabilized and blocked for 20 min with PBS containing $0.1 \%$ Triton ${ }^{\circledR}$ X-100 (BioRad) and 10\% FCS. Wildtype FL5.12 cells growing with or without IL-3 and GFP-alpha globin expressing cells were stained with antihemoglobin polyclonal antibody (ICN Cappel) diluted 1:200 in the blocking solution for 30 min. Cells were washed three times with PBS, then incubated with goat anti-rabbit IgGAlexa Fluor 546 (Molecular Probes) diluted 1:200 in PBS containing 0.1\% Triton X-100 and $1 \%$ FCS for $30 \mathrm{~min}$, and washed three times PBS.

To assess cellular localization of ectopically expressed GATA-2, FL5.12 and NIH3T3 cells were transduced with GFP-GATA-2, FLAG-GATA-2, or GFP, and subsequently sorted using a FACS Calibur (Becton Dickinson). NIH3T3 cells were seeded and grown on cover slips. FL5.12 cells were cytospun on object trays immediately prior immunostaining. FL5.12 and NIH3T3 cells were fixed, permeabilized, and blocked as described above. Wildtype NIH3T3 cells and FLAG-GATA-2 expressing cells were stained with anti-FLAG monoclonal antibody (Sigma) diluted 1:500 in the blocking solution for $30 \mathrm{~min}$. Cells were washed three times with PBS, then incubated with goat anti-mouse IgG-Alexa Fluor 546 diluted 1:200 in PBS containing $0.1 \%$ Triton X-100 and $1 \%$ FCS for $30 \mathrm{~min}$, and finally washed with PBS.

All cells were coverslipped using DAKO mounting medium containing 4',6-diamidino-2phenylindole (DAPI) $(1 \mu \mathrm{g} / \mathrm{ml})$. Cells were analyzed using a Nikon Eclipse TE 2000-S equipped with a Nikon digital camera DXM1200. eGFP was excited at 460 to $500 \mathrm{~nm}$, Alexa Fluor 546 at 510 to $560 \mathrm{~nm}$, and DAPI at 340 to $380 \mathrm{~nm}$. To avoid cross-talk between fluorescence signals and to reject non-specific fluorescence a 510 to $560 \mathrm{~nm}$ band-pass filter was used for eGFP, a $590 \mathrm{~nm}$ filter for Alexa Fluor 546, and a 435 to $485 \mathrm{~nm}$ bandpass filter for DAPI (Chroma Technology Corporation).

\subsection{Determination of heme, hemoglobin, and differentiation stage of FL5.12 cells}

Detection of heme was performed according to the protocol described by Sassa et al. (Sassa, 1976). 1 x $10^{6}$ cells were pelleted, mixed with $0.5 \mathrm{ml} 1 \mathrm{M}$ oxalic acid, and immediately heated for $30 \mathrm{~min}$ at $95^{\circ} \mathrm{C}$. Cells in $1 \mathrm{M}$ oxalic acid without heating served as negative control. After cooling triplicates of $150 \mu \mathrm{l}$ were analyzed fluorometrically using the Analyst ${ }^{\circledR}$ GT Reader (excitation $=400 \mathrm{~nm}$, emission $=662 \mathrm{~nm}$ ). Dilutions of $125 \mathrm{nM}-2.5 \mathrm{nM}$ hemin in a solution of methanol and $0.01 \mathrm{~N} \mathrm{KOH}(1: 1)$, containing $1 \% \mathrm{BSA}$ served to evaluate a standard curve. 
Hemoglobin assay was performed as described by Worthington et al. (Worthington et al., 1987). Briefly, 1 × $10^{6}$ cells were washed twice with PBS, and permeabilized in $500 \mu 1$ water containing $0.01 \%$ Nonidet $^{\circledR}$ P-40 (Fluka). Hemoglobin standard was freshly prepared by dissolving human hemoglobin (Sigma) in water. 2,7-diaminofluorene (DAF), (Sigma) stock solution was prepared by dissolving $10 \mathrm{mg} / \mathrm{ml}$ DAF in $90 \%$ glacial acetic acid. To initiate the color reaction $1.5 \mathrm{ml}$ DAF working solution containing $100 \mu \mathrm{DAF}$ stock solution, $60 \mu \mathrm{l} 30 \%$ hydrogen peroxide, and $6 \mathrm{M}$ urea in $10 \mathrm{ml} 0.1 \mathrm{M}$ Tris buffer, $\mathrm{pH} \mathrm{7,} \mathrm{were} \mathrm{added} \mathrm{to} \mathrm{the} \mathrm{cell}$ suspension or the hemoglobin standards, respectively, vortexed and incubated at room temperature for $10 \mathrm{~min}$. All reactions were performed in polypropylene tubes. Generation of fluorene blue was detected by measuring the absorbance at $610 \mathrm{~nm}$.

The differentiation stage of FL5.12 cells growing with and without IL-3 was evaluated by May-Grünwald-Giemsa staining using Diff-Quik ${ }^{\circledR}$ (Dade, Behring). 



\section{Abbreviations}

$18 \mathrm{~S}$

AHSP

AIF

ALA-S1

AP-1

Apaf-1

AS

AUF-1

$\mathrm{Bcl}$

$\mathrm{BH}$

BSE

C. elegans

CARD

casp

caspase

CBP

CD95

cDNA

ced

c-FLIP

$\mathrm{CFU}$

CHX

cIAP

CML

$\mathrm{CO}$

$\mathrm{CpG}$

CPP32

CRE

CREB

$\mathrm{CS}$

$\mathrm{C}_{\mathrm{T}}$

C-terminal

CYGB

cyt c

DAF

DAPI

DcR

DD

DED
$18 \mathrm{~S}$ ribosomal RNA

alpha globin stabilizing protein

Apoptosis inducing factor

aminolevulinic acid synthase 1

activating protein 1

apoptotic protease activating factor 1

antisense

AU-rich destabilizing elements binding/degradation factor 1

B cell lymphoma

Bcl-2 homology

bovine spongiform encephalopathy

Caenorhabditis elegans

caspase recruitment domain

caspase

cysteine aspartyl protease

CREB-binding protein

cluster of differentiation 95

copy desoxyribonucletidyl acid

cell death defective

cellular FLICE inhibitory protein

colony-forming unit

cycloheximide

cellular inhibitor of apoptosis protein

chronic myeloid leukemia

carbon monoxide

cytosine and guanine connected by a phosphodiester bond

32-kDa putative cysteine protease

$\mathrm{C}$-rich element

cAMP response element-binding protein

cytoskeleton

threshold cycle

carboxy terminal

cytoglobin

cytochrome c

2,7-diaminofluorene

4',6-diamidino-2-phenylindole

decoy receptor

death domain

death effector domain 


\begin{tabular}{|c|c|}
\hline DIABLO & direct IAP binding protein \\
\hline DISC & death-inducing signaling complex \\
\hline DMSO & dimethylsulfoxide \\
\hline DOXO & doxorubicin \\
\hline DR & death receptor \\
\hline EDRF & erythroid differentiation-related factor \\
\hline eGFP & enhanced green fluorescent protein \\
\hline EKLF & erythroid krueppel like factor \\
\hline ER & endoplasmic reticulum \\
\hline ERAF & erythroid association factor \\
\hline ErEN & erythroid enriched endoribonuclease \\
\hline ERK & extracellular signal-regulated kinase \\
\hline FACS & fluorescent activated cell sorting \\
\hline FADD & Fas-associated death domain \\
\hline Fas & Fibroblast-associated \\
\hline FL & fetal liver \\
\hline FOG-1 & Friend of GATA-1 \\
\hline Foxo & forkhead transcription factor \\
\hline GAPDH & glyceraldehyde-3-phosphate dehydrogenase \\
\hline $\mathrm{Hb}$ & hemoglobin \\
\hline Hbb-b1 & beta globin major chain \\
\hline Hbb-b2 & beta globin minor chain \\
\hline $\mathrm{HO}-1$ & heme oxygenase 1 \\
\hline HS & DNase I hypersensitive site \\
\hline Hsp & heat shock protein \\
\hline IAP & inhibitors of apoptosis \\
\hline $\mathrm{ICE}$ & interleukin converting enzyme \\
\hline IL & interleukin \\
\hline IL-3 R $\beta$ & interleukin 3 receptor beta \\
\hline Jak & Janus kinase \\
\hline $\mathrm{kD}$ & kilo Dalton \\
\hline LCR & locus control region \\
\hline $\mathrm{LT}$ & lymphotoxin \\
\hline M & molar \\
\hline M & membrane \\
\hline $\mathrm{m}$ & milli \\
\hline MAPK & mitogen-activated protein kinase \\
\hline $\mathrm{Mb}$ & myoglobin \\
\hline MEK & MAP kinase kinase \\
\hline mRNA & messenger ribonucleic acid \\
\hline $\mathrm{n}$ & nano \\
\hline
\end{tabular}




\begin{tabular}{|c|c|}
\hline $\mathrm{N}$ & nuclear \\
\hline NF I & nuclear factor I \\
\hline NF-E2 & nuclear factor erythroid 2 \\
\hline $\mathrm{NF}-\kappa \mathrm{B}$ & nuclear factor kappa B \\
\hline NGB & neuroglobin \\
\hline nt & nucleotide \\
\hline N-terminal & amino terminal \\
\hline p90S6K & p90 ribosomal S6 kinase \\
\hline PABP & poly-A binding protein \\
\hline PBGD & porphobilinogen deaminase \\
\hline PBS & phosphate buffered saline \\
\hline PDC & programmed cell death \\
\hline PDK 1 & phosphatidylinositol-dependent kinase 1 \\
\hline PFA & paraformaldehyde \\
\hline $\mathrm{PI}(3,4,5) \mathrm{P}_{3}$ & phosphatidylinositol $(3,4,5)$-tris-phosphates \\
\hline $\mathrm{PI}(4,5) \mathrm{P}_{2}$ & phosphatidylinositol $(4,5)$-bisphosphate \\
\hline PI3K & phosphatidylinositol 3-kinase \\
\hline PKB & protein kinase $\mathrm{B}$ \\
\hline PLAD & pre-ligand binding assembly domain \\
\hline PP2A & protein phosphatase $2 \mathrm{~A}$ \\
\hline PTP & permeability transition pore \\
\hline PUMA & p53-upregulated modulator of apoptosis \\
\hline QPCR & quantitative polymerase chain reaction \\
\hline RIP & receptor-interacting protein \\
\hline Rsk & ribosomal S6 kinase \\
\hline RT QPCR & quantitative reverse transcriptase polymerase chain reaction \\
\hline $\mathrm{S}$ & soluble \\
\hline $\mathrm{SD}$ & standard deviation \\
\hline SODD & silencer of death domain \\
\hline STAT & signal transducers and activators of transcription \\
\hline STS & staurosporine \\
\hline $\mathrm{T}$ & tyrosine \\
\hline $\mathrm{TdT}$ & deoxynucleotidyl transferase \\
\hline TM & trans membrane \\
\hline TMR & tetramethylrhodamine \\
\hline TNF $\alpha$ & tumor necrosis factor alpha \\
\hline TNF-R & tumor necrosis factor receptor \\
\hline TRADD & TNF-R-associated death domain \\
\hline TRAF & TNF-R-associated factor \\
\hline TRAIL & TNF-related apoptosis-inducing ligand \\
\hline TRAIL-R & TNF-related apoptosis-inducing ligand receptor \\
\hline
\end{tabular}


TUNEL terminal deoxynucleotidyl transferase dUTP nick end labeling

v-FLIP

viral FLICE inhibitory protein

zVAD-fmk

valine-alanine-aspartic acid fluoromethyl ketone

$\alpha$-complex

alpha-complex

$\alpha \mathrm{CP}$

alpha globin poly(C)-binding protein

$\alpha \mathrm{PRE}$

$\alpha$-globin positive regulatory element 


\section{$7 \quad$ References}

Abreu-Martin,M.T., Vidrich,A., Lynch,D.H., and Targan,S.R. (1995). Divergent induction of apoptosis and IL-8 secretion in HT-29 cells in response to TNF-alpha and ligation of Fas antigen. J. Immunol. 155, 4147-4154.

Adachi,K., Yamaguchi,T., Pang,J., and Surrey,S. (1998). Effects of increased anionic charge in the beta-globin chain on assembly of hemoglobin in vitro. Blood 91, 1438-1445.

Adachi,K., Zhao,Y., and Surrey,S. (2002). Assembly of human hemoglobin (Hb) beta- and gamma-globin chains expressed in a cell-free system with alpha-globin chains to form $\mathrm{Hb} \mathrm{A}$ and $\mathrm{Hb}$ F. J. Biol. Chem. 277 , 13415-13420.

Adams,J.M. and Cory,S. (1998). The Bcl-2 protein family: arbiters of cell survival. Science 281, 1322-1326.

Advani,R., Sorenson,S., Shinar,E., Lande,W., Rachmilewitz,E., and Schrier,S.L. (1992). Characterization and comparison of the red blood cell membrane damage in severe human alpha- and beta-thalassemia. Blood 79, 1058-1063.

Ahmed,M.M. (2004). Regulation of radiation-induced apoptosis by early growth response-1 gene in solid tumors. Curr. Cancer Drug Targets. 4, 43-52.

Algate,P.A., Steelman,L.S., Mayo,M.W., Miyajima,A., and McCubrey,J.A. (1994). Regulation of the interleukin-3 (IL-3) receptor by IL-3 in the fetal liver-derived FL5.12 cell line. Blood 83, 2459-2468.

Alnemri,E.S., Livingston,D.J., Nicholson,D.W., Salvesen,G., Thornberry,N.A., Wong,W.W., and Yuan,J. (1996). Human ICE/CED-3 protease nomenclature. Cell 87, 171.

Anderson,K.P., Crable,S.C., and Lingrel,J.B. (2000). The GATA-E box-GATA motif in the EKLF promoter is required for in vivo expression. Blood 95, 1652-1655.

Andrews,N.C. (1998). The NF-E2 transcription factor. Int. J. Biochem. Cell Biol. 30, 429432.

Anglade,P., Vyas,S., Javoy-Agid,F., Herrero,M.T., Michel,P.P., Marquez,J., MouattPrigent,A., Ruberg,M., Hirsch,E.C., and Agid,Y. (1997). Apoptosis and autophagy in nigral neurons of patients with Parkinson's disease. Histol. Histopathol. 12, 25-31.

Anguita,E., Sharpe,J.A., Sloane-Stanley,J.A., Tufarelli,C., Higgs,D.R., and Wood,W.G. (2002). Deletion of the mouse alpha-globin regulatory element (HS -26) has an unexpectedly mild phenotype. Blood 100, 3450-3456.

Appella,E. and Anderson,C.W. (2001). Post-translational modifications and activation of p53 by genotoxic stresses. Eur. J. Biochem. 268, 2764-2772. 
Aries,A., Trentesaux,C., Ottolenghi,S., Jardillier,J.C., Jeannesson,P., and Doubeikovski,A. (1996). Activation of erythroid-specific promoters during anthracycline-induced differentiation of K562 cells. Blood 87, 2885-2890.

Ashkenazi,A. and Dixit,V.M. (1998). Death receptors: signaling and modulation. Science 281, 1305-1308.

Bank,A., Braverman,A.S., and Marks,P.A. (1969). Globin chain synthesis in thalassemia. Ann. N. Y. Acad. Sci. 165, 231-237.

Basanez,G., Nechushtan,A., Drozhinin,O., Chanturiya,A., Choe,E., Tutt,S., Wood,K.A., Hsu,Y., Zimmerberg,J., and Youle,R.J. (1999). Bax, but not Bcl-xL, decreases the lifetime of planar phospholipid bilayer membranes at subnanomolar concentrations. Proc. Natl. Acad. Sci. U. S. A 96, 5492-5497.

Bera,A., Singh,S., Nagaraj,R., and Vaidya,T. (2003). Induction of autophagic cell death in Leishmania donovani by antimicrobial peptides. Mol. Biochem. Parasitol. 127, 23-35.

Bertrand,R., Solary,E., O'Connor,P., Kohn,K.W., and Pommier,Y. (1994). Induction of a common pathway of apoptosis by staurosporine. Exp. Cell Res. 211, 314-321.

Blalock,W.L., Weinstein-Oppenheimer,C., Chang,F., Hoyle,P.E., Wang,X.Y., Algate,P.A., Franklin,R.A., Oberhaus,S.M., Steelman,L.S., and McCubrey,J.A. (1999). Signal transduction, cell cycle regulatory, and anti-apoptotic pathways regulated by IL-3 in hematopoietic cells: possible sites for intervention with anti-neoplastic drugs. Leukemia 13, 1109-1166.

Blobel,G.A., Nakajima,T., Eckner,R., Montminy,M., and Orkin,S.H. (1998). CREB-binding protein cooperates with transcription factor GATA-1 and is required for erythroid differentiation. Proc. Natl. Acad. Sci. U. S. A 95, 2061-2066.

Bodmer,J.L., Holler,N., Reynard,S., Vinciguerra,P., Schneider,P., Juo,P., Blenis,J., and Tschopp,J. (2000). TRAIL receptor-2 signals apoptosis through FADD and caspase-8. Nat. Cell Biol. 2, 241-243.

Boise,L.H., Gonzalez-Garcia,M., Postema,C.E., Ding,L., Lindsten,T., Turka,L.A., Mao,X., Nunez,G., and Thompson,C.B. (1993). bcl-x, a bcl-2-related gene that functions as a dominant regulator of apoptotic cell death. Cell 74, 597-608.

Bojes,H.K., Feng,X., Kehrer,J.P., and Cohen,G.M. (1999). Apoptosis in hematopoietic cells (FL5.12) caused by interleukin-3 withdrawal: relationship to caspase activity and the loss of glutathione. Cell Death. Differ. 6, 61-70.

Bonni,A., Brunet,A., West,A.E., Datta,S.R., Takasu,M.A., and Greenberg,M.E. (1999). Cell survival promoted by the Ras-MAPK signaling pathway by transcription-dependent and independent mechanisms. Science 286, 1358-1362. 
Borner,C. (2003). The Bcl-2 protein family: sensors and checkpoints for life-or-death decisions. Mol. Immunol. 39, 615-647.

Brachat,A., Pierrat,B., Brungger,A., and Heim,J. (2000). Comparative microarray analysis of gene expression during apoptosis-induction by growth factor deprivation or protein kinase $\mathrm{C}$ inhibition. Oncogene 19, 5073-5082.

Brachat,A., Pierrat,B., Xynos,A., Brecht,K., Simonen,M., Brungger,A., and Heim,J. (2002). A microarray-based, integrated approach to identify novel regulators of cancer drug response and apoptosis. Oncogene 21, 8361-8371.

Braun,H., Koop,R., Ertmer,A., Nacht,S., and Suske,G. (2001). Transcription factor Sp3 is regulated by acetylation. Nucleic Acids Res. 29, 4994-5000.

Brazil,D.P. and Hemmings,B.A. (2001). Ten years of protein kinase B signalling: a hard Akt to follow. Trends Biochem. Sci. 26, 657-664.

Brouard,S., Otterbein,L.E., Anrather,J., Tobiasch,E., Bach,F.H., Choi,A.M., and Soares,M.P. (2000). Carbon monoxide generated by heme oxygenase 1 suppresses endothelial cell apoptosis. J. Exp. Med. 192, 1015-1026.

Brunet,A., Bonni,A., Zigmond,M.J., Lin,M.Z., Juo,P., Hu,L.S., Anderson,M.J., Arden,K.C., Blenis,J., and Greenberg,M.E. (1999). Akt promotes cell survival by phosphorylating and inhibiting a Forkhead transcription factor. Cell 96, 857-868.

Buckle,V.J., Higgs,D.R., Wilkie,A.O., Super,M., and Weatherall,D.J. (1988). Localisation of human alpha globin to 16p13.3----pter. J. Med. Genet. 25, 847-849.

Burmester,T., Ebner,B., Weich,B., and Hankeln,T. (2002). Cytoglobin: a novel globin type ubiquitously expressed in vertebrate tissues. Mol. Biol. Evol. 19, 416-421.

Burmester,T., Weich,B., Reinhardt,S., and Hankeln,T. (2000). A vertebrate globin expressed in the brain. Nature 407, 520-523.

Bursch,W., Ellinger,A., Kienzl,H., Torok,L., Pandey,S., Sikorska,M., Walker,R., and Hermann,R.S. (1996). Active cell death induced by the anti-estrogens tamoxifen and ICI 164 384 in human mammary carcinoma cells (MCF-7) in culture: the role of autophagy. Carcinogenesis 17, 1595-1607.

Butt,A.J., Harvey,N.L., Parasivam,G., and Kumar,S. (1998). Dimerization and autoprocessing of the Nedd2 (caspase-2) precursor requires both the prodomain and the carboxyl-terminal regions. J. Biol. Chem. 273, 6763-6768.

Cao,A. and Moi,P. (2002). Regulation of the globin genes. Pediatr. Res. 51, 415-421. 
Cardone,M.H., Roy,N., Stennicke,H.R., Salvesen,G.S., Franke,T.F., Stanbridge,E., Frisch,S., and Reed,J.C. (1998). Regulation of cell death protease caspase-9 by phosphorylation. Science 282, 1318-1321.

Cascino,I., Fiucci,G., Papoff,G., and Ruberti,G. (1995). Three functional soluble forms of the human apoptosis-inducing Fas molecule are produced by alternative splicing. J. Immunol. 154, 2706-2713.

Cerretti,D.P., Kozlosky,C.J., Mosley,B., Nelson,N., Van Ness,K., Greenstreet,T.A., March,C.J., Kronheim,S.R., Druck,T., Cannizzaro,L.A., and . (1992). Molecular cloning of the interleukin-1 beta converting enzyme. Science 256, 97-100.

Chan,F.K., Chun,H.J., Zheng,L., Siegel,R.M., Bui,K.L., and Lenardo,M.J. (2000). A domain in TNF receptors that mediates ligand-independent receptor assembly and signaling. Science $288,2351-2354$.

Chinnaiyan,A.M., O'Rourke,K., Lane,B.R., and Dixit,V.M. (1997). Interaction of CED-4 with CED-3 and CED-9: a molecular framework for cell death. Science 275, 1122-1126.

Chittenden,T., Flemington,C., Houghton,A.B., Ebb,R.G., Gallo,G.J., Elangovan,B., Chinnadurai,G., and Lutz,R.J. (1995). A conserved domain in Bak, distinct from BH1 and BH2, mediates cell death and protein binding functions. EMBO J. 14, 5589-5596.

Cirman,T., Oresic,K., Mazovec,G.D., Turk,V., Reed,J.C., Myers,R.M., Salvesen,G.S., and Turk,B. (2004). Selective disruption of lysosomes in HeLa cells triggers apoptosis mediated by cleavage of Bid by multiple papain-like lysosomal cathepsins. J. Biol. Chem. 279, 35783587.

Coleman,T.R., Fishkind,D.J., Mooseker,M.S., and Morrow,J.S. (1989). Functional diversity among spectrin isoforms. Cell Motil. Cytoskeleton 12, 225-247.

Cowling,V. and Downward,J. (2002). Caspase-6 is the direct activator of caspase- 8 in the cytochrome c-induced apoptosis pathway: absolute requirement for removal of caspase-6 prodomain. Cell Death. Differ. 9, 1046-1056.

Coyle,B., Freathy,C., Gant,T.W., Roberts,R.A., and Cain,K. (2003). Characterization of the transforming growth factor-beta 1-induced apoptotic transcriptome in $\mathrm{FaO}$ hepatoma cells. J. Biol. Chem. 278, 5920-5928.

Crossley,M., Merika,M., and Orkin,S.H. (1995). Self-association of the erythroid transcription factor GATA-1 mediated by its zinc finger domains. Mol. Cell Biol. 15, 24482456.

Curtin,P.T., Liu,D.P., Liu,W., Chang,J.C., and Kan,Y.W. (1989). Human beta-globin gene expression in transgenic mice is enhanced by a distant DNase I hypersensitive site. Proc. Natl. Acad. Sci. U. S. A $86,7082-7086$. 
Darmon,A.J., Nicholson,D.W., and Bleackley,R.C. (1995). Activation of the apoptotic protease CPP32 by cytotoxic T-cell-derived granzyme B. Nature 377, 446-448.

Datta,S.R., Dudek,H., Tao,X., Masters,S., Fu,H., Gotoh,Y., and Greenberg,M.E. (1997). Akt phosphorylation of BAD couples survival signals to the cell-intrinsic death machinery. Cell 91, 231-241.

Davie,J.R. and Spencer,V.A. (1999). Control of histone modifications. J. Cell Biochem. Suppl 32-33:141-8., 141-148.

De Maria,R., Zeuner,A., Eramo,A., Domenichelli,C., Bonci,D., Grignani,F., Srinivasula,S.M., Alnemri,E.S., Testa,U., and Peschle,C. (1999). Negative regulation of erythropoiesis by caspase-mediated cleavage of GATA-1. Nature 401, 489-493.

Dedoussis,G.V., $\quad$ Mouzaki,A., Theodoropoulou,M., $\quad$ Menounos,P., $\quad$ Kyrtsonis,M.C., Karameris,A., and Maniatis,A. (1999). Endogenous interleukin 6 conveys resistance to cisdiamminedichloroplatinum-mediated apoptosis of the K562 human leukemic cell line. Exp. Cell Res. 249, 269-278.

Deiss,L.P., Galinka,H., Berissi,H., Cohen,O., and Kimchi,A. (1996). Cathepsin D protease mediates programmed cell death induced by interferon-gamma, Fas/APO-1 and TNF-alpha. EMBO J. 15, 3861-3870.

Desagher,S., Osen-Sand,A., Nichols,A., Eskes,R., Montessuit,S., Lauper,S., Maundrell,K., Antonsson,B., and Martinou,J.C. (1999). Bid-induced conformational change of Bax is responsible for mitochondrial cytochrome c release during apoptosis. J. Cell Biol. 144, 891901.

Devireddy,L.R., Teodoro,J.G., Richard,F.A., and Green,M.R. (2001). Induction of apoptosis by a secreted lipocalin that is transcriptionally regulated by IL-3 deprivation. Science 293, 829-834.

Dong,Z., Saikumar,P., Weinberg,J.M., and Venkatachalam,M.A. (1997). Internucleosomal DNA cleavage triggered by plasma membrane damage during necrotic cell death. Involvement of serine but not cysteine proteases. Am. J. Pathol. 151, 1205-1213.

Dow, Lindsay, and Morrison (1995). Haemoglobin - structure and function. In Biochemistry, Hogg and Grove, eds. Addison; Wesley), pp. 369-376.

Dubart,A., Romeo,P.H., Vainchenker,W., and Dumenil,D. (1996). Constitutive expression of GATA-1 interferes with the cell-cycle regulation. Blood 87, 3711-3721.

Dunn,W.A., Jr. (1990). Studies on the mechanisms of autophagy: formation of the autophagic vacuole. J. Cell Biol. 110, 1923-1933. 
E.Rachmilewitz and St.Schrier (2001). Pathophysiology of beta-thalassemia. In Disorders of hemoglobin: Genetics, pathophysiology, and clinical management, Steinberg, Forget, Higgs, and Nagel, eds. (Cambrigde: Cambridge University Press), pp. 233-251.

Earnshaw,W.C., Martins,L.M., and Kaufmann,S.H. (1999). Mammalian caspases: structure, activation, substrates, and functions during apoptosis. Annu. Rev. Biochem. 68:383-424., 383-424.

Ellis,H.M. and Horvitz,H.R. (1986). Genetic control of programmed cell death in the nematode C. elegans. Cell 44, 817-829.

Eskes,R., Desagher,S., Antonsson,B., and Martinou,J.C. (2000). Bid induces the oligomerization and insertion of Bax into the outer mitochondrial membrane. Mol. Cell Biol. 20,929-935.

Ewing,J.F. and Maines,M.D. (1991). Rapid induction of heme oxygenase 1 mRNA and protein by hyperthermia in rat brain: heme oxygenase 2 is not a heat shock protein. Proc. Natl. Acad. Sci. U. S. A 88, 5364-5368.

Fadok,V.A., Savill,J.S., Haslett,C., Bratton,D.L., Doherty,D.E., Campbell,P.A., and Henson,P.M. (1992). Different populations of macrophages use either the vitronectin receptor or the phosphatidylserine receptor to recognize and remove apoptotic cells. J. Immunol. 149, 4029-4035.

Feng,D.X., Liu,D.P., Huang,Y., Wu,L., Li,T.C., Wu,M., Tang,X.B., and Liang,C.C. (2001). The expression of human alpha -like globin genes in transgenic mice mediated by bacterial artificial chromosome. Proc. Natl. Acad. Sci. U. S. A 98, 15073-15077.

Ferris,C.D., Jaffrey,S.R., Sawa,A., Takahashi,M., Brady,S.D., Barrow,R.K., Tysoe,S.A., Wolosker,H., Baranano,D.E., Dore,S., Poss,K.D., and Snyder,S.H. (1999). Haem oxygenase-1 prevents cell death by regulating cellular iron. Nat. Cell Biol. 1, 152-157.

Fessas,P., Loukopoulos,D., and Kaltsoya,A. (1966). Peptide analysis of the inclusions of erythroid cells in beta-thalassemia. Biochim. Biophys. Acta 124, 430-432.

Finucane,D.M., Bossy-Wetzel,E., Waterhouse,N.J., Cotter,T.G., and Green,D.R. (1999). Baxinduced caspase activation and apoptosis via cytochrome c release from mitochondria is inhibitable by Bcl-xL. J. Biol. Chem. 274, 2225-2233.

Flogel,U., Merx,M.W., Godecke,A., Decking,U.K., and Schrader,J. (2001). Myoglobin: A scavenger of bioactive NO. Proc. Natl. Acad. Sci. U. S. A 98, 735-740.

Fordel,E., Geuens,E., Dewilde,S., Rottiers,P., Carmeliet,P., Grooten,J., and Moens,L. (2004). Cytoglobin expression is upregulated in all tissues upon hypoxia: an in vitro and in vivo study by quantitative real-time PCR. Biochem. Biophys. Res. Commun. 319, 342-348. 
Fortier,N., Snyder,L.M., Garver,F., Kiefer,C., McKenney,J., and Mohandas,N. (1988). The relationship between in vivo generated hemoglobin skeletal protein complex and increased red cell membrane rigidity. Blood 71, 1427-1431.

Fransen,L., Muller,R., Marmenout,A., Tavernier,J., Van der,H.J., Kawashima,E., Chollet,A., Tizard,R., Van Heuverswyn,H., Van Vliet,A., and . (1985). Molecular cloning of mouse tumour necrosis factor cDNA and its eukaryotic expression. Nucleic Acids Res. 13, 44174429 .

Fulda,S., Meyer,E., and Debatin,K.M. (2000). Metabolic inhibitors sensitize for CD95 (APO1/Fas)-induced apoptosis by down-regulating Fas-associated death domain-like interleukin 1converting enzyme inhibitory protein expression. Cancer Res. 60, 3947-3956.

Gell,D., Kong,Y., Eaton,S.A., Weiss,M.J., and Mackay,J.P. (2002). Biophysical Characterization of the alpha -Globin Binding Protein alpha -Hemoglobin Stabilizing Protein. J. Biol. Chem. 277, 40602-40609.

Geuens,E., Brouns,I., Flamez,D., Dewilde,S., Timmermans,J.P., and Moens,L. (2003). A globin in the nucleus! J. Biol. Chem. 278, 30417-30420.

Gil,J. and Esteban,M. (2000). Induction of apoptosis by the dsRNA-dependent protein kinase (PKR): mechanism of action. Apoptosis. 5, 107-114.

Goldstein,J.C., Waterhouse,N.J., Juin,P., Evan,G.I., and Green,D.R. (2000). The coordinate release of cytochrome c during apoptosis is rapid, complete and kinetically invariant. Nat. Cell Biol. 2, 156-162.

Gomez-Angelats,M. and Cidlowski,J.A. (2003). Molecular evidence for the nuclear localization of FADD. Cell Death. Differ. 10, 791-797.

Gonzalez-Garcia,M., Perez-Ballestero,R., Ding,L., Duan,L., Boise,L.H., Thompson,C.B., and Nunez,G. (1994). bcl-XL is the major bcl-x mRNA form expressed during murine development and its product localizes to mitochondria. Development 120, 3033-3042.

Green,D. and Kroemer,G. (1998). The central executioners of apoptosis: caspases or mitochondria? Trends Cell Biol. 8, 267-271.

Green,D.R. (1998). Apoptotic pathways: the roads to ruin. Cell 94, 695-698.

Gregory,C.D., Dive,C., Henderson,S., Smith,C.A., Williams,G.T., Gordon,J., and Rickinson,A.B. (1991). Activation of Epstein-Barr virus latent genes protects human B cells from death by apoptosis. Nature 349, 612-614.

Grell,M., Zimmermann,G., Gottfried,E., Chen,C.M., Grunwald,U., Huang,D.C., Wu Lee,Y.H., Durkop,H., Engelmann,H., Scheurich,P., Wajant,H., and Strasser,A. (1999). Induction of cell death by tumour necrosis factor (TNF) receptor 2, CD40 and CD30: a role for TNF-R1 activation by endogenous membrane-anchored TNF. EMBO J. 18, 3034-3043. 
Gross,A., Jockel,J., Wei,M.C., and Korsmeyer,S.J. (1998). Enforced dimerization of BAX results in its translocation, mitochondrial dysfunction and apoptosis. EMBO J. 17, 3878-3885.

Hardison,R. (1998). Hemoglobins from bacteria to man: evolution of different patterns of gene expression. J. Exp. Biol. 201, 1099-1117.

Hardison,R.C. (1996). A brief history of hemoglobins: plant, animal, protist, and bacteria. Proc. Natl. Acad. Sci. U. S. A 93, 5675-5679.

Hardison,R.C., Sawada,I., Cheng,J.F., Shen,C.K., and Schmid,C.W. (1986). A previously undetected pseudogene in the human alpha globin gene cluster. Nucleic Acids Res. 14, 19031911.

Harvey,N.L., Butt,A.J., and Kumar,S. (1997). Functional activation of Nedd2/ICH-1 (caspase-2) is an early process in apoptosis. J. Biol. Chem. 272, 13134-13139.

Haughn,L., Hawley,R.G., Morrison,D.K., von Boehmer,H., and Hockenbery,D.M. (2003). BCL-2 and BCL-XL restrict lineage choice during hematopoietic differentiation. J. Biol. Chem. 278, 25158-25165.

He,H., Lam,M., McCormick,T.S., and Distelhorst,C.W. (1997). Maintenance of calcium homeostasis in the endoplasmic reticulum by Bcl-2. J. Cell Biol. 138, 1219-1228.

Hengartner,M.O., Ellis,R.E., and Horvitz,H.R. (1992). Caenorhabditis elegans gene ced-9 protects cells from programmed cell death. Nature 356, 494-499.

Hershko,T. and Ginsberg,D. (2004). Up-regulation of Bcl-2 homology 3 (BH3)-only proteins by E2F1 mediates apoptosis. J. Biol. Chem. 279, 8627-8634.

Heyworth,C., Gale,K., Dexter,M., May,G., and Enver,T. (1999). A GATA-2/estrogen receptor chimera functions as a ligand-dependent negative regulator of self-renewal. Genes Dev. 13, 1847-1860.

Higgs,D.R., Wood,W.G., Jarman,A.P., Sharpe,J., Lida,J., Pretorius,I.M., and Ayyub,H. (1990). A major positive regulatory region located far upstream of the human alpha-globin gene locus. Genes Dev. 4, 1588-1601.

Hill,M.M., Adrain,C., Duriez,P.J., Creagh,E.M., and Martin,S.J. (2004). Analysis of the composition, assembly kinetics and activity of native Apaf-1 apoptosomes. EMBO J. 23, 2134-2145.

Hofmann,K., Bucher,P., and Tschopp,J. (1997). The CARD domain: a new apoptotic signalling motif. Trends Biochem. Sci. 22, 155-156.

Hsu,Y.T., Wolter,K.G., and Youle,R.J. (1997). Cytosol-to-membrane redistribution of Bax and Bcl-X(L) during apoptosis. Proc. Natl. Acad. Sci. U. S. A 94, 3668-3672. 
Ikonomi,P., Noguchi,C.T., Miller,W., Kassahun,H., Hardison,R., and Schechter,A.N. (2000). Levels of GATA-1/GATA-2 transcription factors modulate expression of embryonic and fetal hemoglobins. Gene 261, 277-287.

Ingram,V.M. (1989). Abnormal human haemoglobins. I. The comparison of normal human and sickle-cell haemoglobins by "fingerprinting". 1958. Biochim. Biophys. Acta 1000:151-7., 151-157.

Irmler,M., Thome,M., Hahne,M., Schneider,P., Hofmann,K., Steiner,V., Bodmer,J.L., Schroter,M., Burns,K., Mattmann,C., Rimoldi,D., French,L.E., and Tschopp,J. (1997). Inhibition of death receptor signals by cellular FLIP. Nature 388, 190-195.

Itoh,N. and Nagata,S. (1993). A novel protein domain required for apoptosis. Mutational analysis of human Fas antigen. J. Biol. Chem. 268, 10932-10937.

Iwasaki,H., Mizuno,S., Wells,R.A., Cantor,A.B., Watanabe,S., and Akashi,K. (2003). GATA1 converts lymphoid and myelomonocytic progenitors into the megakaryocyte/erythrocyte lineages. Immunity. 19, 451-462.

Jiang,S., Cai,J., Wallace,D.C., and Jones,D.P. (1999). Cytochrome c-mediated apoptosis in cells lacking mitochondrial DNA. Signaling pathway involving release and caspase 3 activation is conserved. J. Biol. Chem. 274, 29905-29911.

Johnson,B.W. and Boise,L.H. (1999). Bcl-2 and caspase inhibition cooperate to inhibit tumor necrosis factor-alpha-induced cell death in a Bcl-2 cleavage-independent fashion. J. Biol. Chem. 274, 18552-18558.

Johnson,D.E. (1998). Regulation of survival pathways by IL-3 and induction of apoptosis following IL-3 withdrawal. Front Biosci. 3:d313-24., d313-d324.

Kannan,R., Labotka,R., and Low,P.S. (1988). Isolation and characterization of the hemichrome-stabilized membrane protein aggregates from sickle erythrocytes. Major site of autologous antibody binding. J. Biol. Chem. 263, 13766-13773.

Kerr,J.F., Wyllie,A.H., and Currie,A.R. (1972). Apoptosis: a basic biological phenomenon with wide-ranging implications in tissue kinetics. Br. J. Cancer 26, 239-257.

Kihm,A.J., Kong,Y., Hong,W., Russell,J.E., Rouda,S., Adachi,K., Simon,M.C., Blobel,G.A., and Weiss,M.J. (2002). An abundant erythroid protein that stabilizes free alpha-haemoglobin. Nature 417, 758-763.

Kirsch,D.G., Doseff,A., Chau,B.N., Lim,D.S., Souza-Pinto,N.C., Hansford,R., Kastan,M.B., Lazebnik,Y.A., and Hardwick,J.M. (1999). Caspase-3-dependent cleavage of Bcl-2 promotes release of cytochrome c. J. Biol. Chem. 274, 21155-21161. 
Kischkel,F.C., Hellbardt,S., Behrmann,I., Germer,M., Pawlita,M., Krammer,P.H., and Peter,M.E. (1995). Cytotoxicity-dependent APO-1 (Fas/CD95)-associated proteins form a death-inducing signaling complex (DISC) with the receptor. EMBO J. 14, 5579-5588.

Kischkel,F.C., Lawrence,D.A., Tinel,A., LeBlanc,H., Virmani,A., Schow,P., Gazdar,A., Blenis,J., Arnott,D., and Ashkenazi,A. (2001). Death receptor recruitment of endogenous caspase-10 and apoptosis initiation in the absence of caspase-8. J. Biol. Chem. 276, 4663946646.

Klionsky,D.J. and Emr,S.D. (2000). Autophagy as a regulated pathway of cellular degradation. Science 290, 1717-1721.

Konkel,D.A., Maizel,J.V., Jr., and Leder,P. (1979). The evolution and sequence comparison of two recently diverged mouse chromosomal beta--globin genes. Cell 18, 865-873.

Krajewski,S., Tanaka,S., Takayama,S., Schibler,M.J., Fenton,W., and Reed,J.C. (1993). Investigation of the subcellular distribution of the bcl-2 oncoprotein: residence in the nuclear envelope, endoplasmic reticulum, and outer mitochondrial membranes. Cancer Res. 53, 47014714.

Kumar,S. (1995). Inhibition of apoptosis by the expression of antisense Nedd2. FEBS Lett. $368,69-72$.

Kuwana,T., Smith,J.J., Muzio,M., Dixit,V., Newmeyer,D.D., and Kornbluth,S. (1998). Apoptosis induction by caspase- 8 is amplified through the mitochondrial release of cytochrome c. J. Biol. Chem. 273, 16589-16594.

Lassus,P., Opitz-Araya,X., and Lazebnik,Y. (2002). Requirement for caspase-2 in stressinduced apoptosis before mitochondrial permeabilization. Science 297, 1352-1354.

Laverriere,A.C., MacNeill,C., Mueller,C., Poelmann,R.E., Burch,J.B., and Evans,T. (1994). GATA-4/5/6, a subfamily of three transcription factors transcribed in developing heart and gut. J. Biol. Chem. 269, 23177-23184.

Lee,H.H., Dadgostar,H., Cheng,Q., Shu,J., and Cheng,G. (1999). NF-kappaB-mediated upregulation of $\mathrm{Bcl}-\mathrm{x}$ and $\mathrm{Bfl}-1 / \mathrm{A} 1$ is required for $\mathrm{CD} 40$ survival signaling in B lymphocytes. Proc. Natl. Acad. Sci. U. S. A 96, 9136-9141.

Lewis,R.A., Kay,R.W., and Hathorn,M. (1966). Sickle cell disease and glucose-6-phosphate dehydrogenase. Acta Haematol. 36, 399-411.

Li,P., Nijhawan,D., Budihardjo,I., Srinivasula,S.M., Ahmad,M., Alnemri,E.S., and Wang,X. (1997). Cytochrome c and dATP-dependent formation of Apaf-1/caspase-9 complex initiates an apoptotic protease cascade. Cell 91, 479-489.

Lim,L.C., Swendeman,S.L., and Sheffery,M. (1992). Molecular cloning of the alpha-globin transcription factor CP2. Mol. Cell Biol. 12, 828-835. 
Lippens,S., VandenBroecke,C., Van Damme,E., Tschachler,E., Vandenabeele,P., and Declercq,W. (2003). Caspase-14 is expressed in the epidermis, the choroid plexus, the retinal pigment epithelium and thymic Hassall's bodies. Cell Death. Differ. 10, 257-259.

Liu,J.J., Hou,S.C., and Shen,C.K. (2003). Erythroid gene suppression by NF-kappa B. J. Biol. Chem. 278, 19534-19540.

Liu,X., Kim,C.N., Yang,J., Jemmerson,R., and Wang,X. (1996). Induction of apoptotic program in cell-free extracts: requirement for dATP and cytochrome c. Cell 86, 147-157.

Lowrey,C.H., Bodine,D.M., and Nienhuis,A.W. (1992). Mechanism of DNase I hypersensitive site formation within the human globin locus control region. Proc. Natl. Acad. Sci. U. S. A 89, 1143-1147.

Loyd,M.R., Okamoto,Y., Randall,M.S., and Ney,P.A. (2003). Role of AP1/NFE2 binding sites in endogenous alpha-globin gene transcription. Blood 102, 4223-4228.

Luo,X., Budihardjo,I., Zou,H., Slaughter,C., and Wang,X. (1998). Bid, a Bcl2 interacting protein, mediates cytochrome $\mathrm{c}$ release from mitochondria in response to activation of cell surface death receptors. Cell 94, 481-490.

Luzzatto,L. and Notaro,R. (2002). Haemoglobin's chaperone. Nature 417, 703-705.

Mahadevappa,M. and Warrington,J.A. (1999). A high-density probe array sample preparation method using 10- to 100-fold fewer cells. Nat. Biotechnol. 17, 1134-1136.

Martin,F., van Deursen,J.M., Shivdasani,R.A., Jackson,C.W., Troutman,A.G., and Ney,P.A. (1998). Erythroid maturation and globin gene expression in mice with combined deficiency of NF-E2 and nrf-2. Blood 91, 3459-3466.

Martinou,J.C., Desagher,S., and Antonsson,B. (2000). Cytochrome c release from mitochondria: all or nothing. Nat. Cell Biol. 2, E41-E43.

Marzo,I., Brenner,C., Zamzami,N., Jurgensmeier,J.M., Susin,S.A., Vieira,H.L., Prevost,M.C., Xie,Z., Matsuyama,S., Reed,J.C., and Kroemer,G. (1998). Bax and adenine nucleotide translocator cooperate in the mitochondrial control of apoptosis. Science 281, 2027-2031.

Mc Gee,M.M., Campiani,G., Ramunno,A., Fattorusso,C., Nacci,V., Lawler,M., Williams,D.C., and Zisterer,D.M. (2001). Pyrrolo-1,5-benzoxazepines induce apoptosis in chronic myelogenous leukemia (CML) cells by bypassing the apoptotic suppressor bcr-abl. J. Pharmacol. Exp. Ther. 296, 31-40.

McCoubrey,W.K., Jr., Huang,T.J., and Maines,M.D. (1997). Isolation and characterization of a cDNA from the rat brain that encodes hemoprotein heme oxygenase-3. Eur. J. Biochem. 247, 725-732. 
McCubrey,J., Holland,G., McKearn,J., and Risser,R. (1989). Abrogation of factordependence in two IL-3-dependent cell lines can occur by two distinct mechanisms. Oncogene Res. 4, 97-109.

McKearn,J.P., McCubrey,J., and Fagg,B. (1985). Enrichment of hematopoietic precursor cells and cloning of multipotential B-lymphocyte precursors. Proc. Natl. Acad. Sci. U. S. A 82, 7414-7418.

Melino,G., Bernassola,F., Ranalli,M., Yee,K., Zong,W.X., Corazzari,M., Knight,R.A., Green,D.R., Thompson,C., and Vousden,K.H. (2004). p73 Induces apoptosis via PUMA transactivation and Bax mitochondrial translocation. J. Biol. Chem. 279, 8076-8083.

Merika,M. and Orkin,S.H. (1995). Functional synergy and physical interactions of the erythroid transcription factor GATA-1 with the Kruppel family proteins Sp1 and EKLF. Mol. Cell Biol. 15, 2437-2447.

Miele,G., Manson,J., and Clinton,M. (2001). A novel erythroid-specific marker of transmissible spongiform encephalopathies. Nat. Med. 7, 361-364.

Minshall,C., Arkins,S., Freund,G.G., and Kelley,K.W. (1996). Requirement for phosphatidylinositol 3'-kinase to protect hemopoietic progenitors against apoptosis depends upon the extracellular survival factor. J. Immunol. 156, 939-947.

Miyashita,T. and Reed,J.C. (1995). Tumor suppressor p53 is a direct transcriptional activator of the human bax gene. Cell 80, 293-299.

Molkentin,J.D. (2000). The zinc finger-containing transcription factors GATA-4, -5, and -6. Ubiquitously expressed regulators of tissue-specific gene expression. J. Biol. Chem. 275, 38949-38952.

Mui,A.L., Wakao,H., O'Farrell,A.M., Harada,N., and Miyajima,A. (1995). Interleukin-3, granulocyte-macrophage colony stimulating factor and interleukin-5 transduce signals through two STAT5 homologs. EMBO J. 14, 1166-1175.

Muirhead,H., Cox,J.M., Mazzarella,L., and Perutz,M.F. (1967). Structure and function of haemoglobin. 3. A three-dimensional fourier synthesis of human deoxyhaemoglobin at 5.5 Angstrom resolution. J. Mol. Biol. 28, 117-156.

Murphy,K.M., Ranganathan,V., Farnsworth,M.L., Kavallaris,M., and Lock,R.B. (2000). Bcl2 inhibits Bax translocation from cytosol to mitochondria during drug-induced apoptosis of human tumor cells. Cell Death. Differ. 7, 102-111.

Muzio,M., Chinnaiyan,A.M., Kischkel,F.C., O'Rourke,K., Shevchenko,A., Ni,J., Scaffidi,C., Bretz,J.D., Zhang,M., Gentz,R., Mann,M., Krammer,P.H., Peter,M.E., and Dixit,V.M. (1996). FLICE, a novel FADD-homologous ICE/CED-3-like protease, is recruited to the CD95 (Fas/APO-1) death--inducing signaling complex. Cell 85, 817-827. 
Myers,R.M., Tilly,K., and Maniatis,T. (1986). Fine structure genetic analysis of a beta-globin promoter. Science 232, 613-618.

Nakagawa,T., Zhu,H., Morishima,N., Li,E., Xu,J., Yankner,B.A., and Yuan,J. (2000). Caspase-12 mediates endoplasmic-reticulum-specific apoptosis and cytotoxicity by amyloidbeta. Nature 403, 98-103.

Nakano,K. and Vousden,K.H. (2001). PUMA, a novel proapoptotic gene, is induced by p53. Mol. Cell 7, 683-694.

Nguyen,M., Millar,D.G., Yong,V.W., Korsmeyer,S.J., and Shore,G.C. (1993). Targeting of $\mathrm{Bcl}-2$ to the mitochondrial outer membrane by a $\mathrm{COOH}$-terminal signal anchor sequence. $\mathrm{J}$. Biol. Chem. 268, 25265-25268.

Ni,R., Tomita,Y., Matsuda,K., Ichihara,A., Ishimura,K., Ogasawara,J., and Nagata,S. (1994). Fas-mediated apoptosis in primary cultured mouse hepatocytes. Exp. Cell Res. 215, 332-337.

Nimmanapalli,R., Porosnicu,M., Nguyen,D., Worthington,E., O'Bryan,E., Perkins,C., and Bhalla,K. (2001). Cotreatment with STI-571 enhances tumor necrosis factor alpha-related apoptosis-inducing ligand (TRAIL or apo-2L)-induced apoptosis of Bcr-Abl-positive human acute leukemia cells. Clin. Cancer Res. 7, 350-357.

Nyoung,M.N., Trentesaux,C., Aries,A., Carpentier,Y., Jardillier,J.C., Gorisse,M.C., and Jeannesson,P. (1994). Effect of aclacinomycin-doxorubicin association on differentiation and growth of human erythroleukemic K562 cells. Anticancer Res. 14, 1203-1208.

Oda,E., Ohki,R., Murasawa,H., Nemoto,J., Shibue,T., Yamashita,T., Tokino,T., Taniguchi,T., and Tanaka,N. (2000). Noxa, a BH3-only member of the Bcl-2 family and candidate mediator of p53-induced apoptosis. Science 288, 1053-1058.

Ohneda,K. and Yamamoto,M. (2002). Roles of hematopoietic transcription factors GATA-1 and GATA-2 in the development of red blood cell lineage. Acta Haematol. 108, 237-245.

Oltvai,Z.N., Milliman,C.L., and Korsmeyer,S.J. (1993). Bcl-2 heterodimerizes in vivo with a conserved homolog, Bax, that accelerates programmed cell death. Cell 74, 609-619.

Orkin,S.H. (1992). GATA-binding transcription factors in hematopoietic cells. Blood 80, 575581.

Orkin,S.H. (1995). Hematopoiesis: how does it happen? Curr. Opin. Cell Biol. 7, 870-877.

Pan,G., Ni,J., Wei,Y.F., Yu,G., Gentz,R., and Dixit,V.M. (1997). An antagonist decoy receptor and a death domain-containing receptor for TRAIL. Science 277, 815-818.

Pan,G., O'Rourke,K., and Dixit,V.M. (1998). Caspase-9, Bcl-XL, and Apaf-1 form a ternary complex. J. Biol. Chem. 273, 5841-5845. 
Persons,D.A., Allay,J.A., Allay,E.R., Ashmun,R.A., Orlic,D., Jane,S.M., Cunningham,J.M., and Nienhuis,A.W. (1999). Enforced expression of the GATA-2 transcription factor blocks normal hematopoiesis. Blood 93, 488-499.

Pitti,R.M., Marsters,S.A., Lawrence,D.A., Roy,M., Kischkel,F.C., Dowd,P., Huang,A., Donahue,C.J., Sherwood,S.W., Baldwin,D.T., Godowski,P.J., Wood,W.I., Gurney,A.L., Hillan,K.J., Cohen,R.L., Goddard,A.D., Botstein,D., and Ashkenazi,A. (1998). Genomic amplification of a decoy receptor for Fas ligand in lung and colon cancer. Nature 396, 699703.

Pondel,M.D., Murphy,S., Pearson,L., Craddock,C., and Proudfoot,N.J. (1995). Sp1 functions in a chromatin-dependent manner to augment human alpha-globin promoter activity. Proc. Natl. Acad. Sci. U. S. A $92,7237-7241$.

Ponka,P. (1997). Tissue-specific regulation of iron metabolism and heme synthesis: distinct control mechanisms in erythroid cells. Blood 89, 1-25.

Porter,A.G. and Janicke,R.U. (1999). Emerging roles of caspase-3 in apoptosis. Cell Death. Differ. 6, 99-104.

Pravdenkova,S.V., Basnakian,A.G., James,S.J., and Andersen,B.J. (1996). DNA fragmentation and nuclear endonuclease activity in rat brain after severe closed head injury. Brain Res. 729, 151-155.

Proudfoot,N.J. and Maniatis,T. (1980). The structure of a human alpha-globin pseudogene and its relationship to alpha-globin gene duplication. Cell 21, 537-544.

Rabbitts,T.H. (1976). Bacterial cloning of plasmids carrying copies of rabbit globin messenger RNA. Nature 260, 221-225.

Rao,L., Perez,D., and White,E. (1996). Lamin proteolysis facilitates nuclear events during apoptosis. J. Cell Biol. 135, 1441-1455.

Ricchetti,G.A., Williams,L.M., and Foxwell,B.M. (2004). Heme oxygenase 1 expression induced by IL-10 requires STAT-3 and phosphoinositol-3 kinase and is inhibited by lipopolysaccharide. J. Leukoc. Biol. . .

Richter,C., Schweizer,M., Cossarizza,A., and Franceschi,C. (1996). Control of apoptosis by the cellular ATP level. FEBS Lett. 378, 107-110.

Robertson,J.D., Gogvadze,V., Kropotov,A., Vakifahmetoglu,H., Zhivotovsky,B., and Orrenius,S. (2004). Processed caspase-2 can induce mitochondria-mediated apoptosis independently of its enzymatic activity. EMBO Rep. 5, 643-648.

Rodgers,N.D., Wang,Z., and Kiledjian,M. (2002). Characterization and purification of a mammalian endoribonuclease specific for the alpha -globin mRNA. J. Biol. Chem. 277, 25972604. 
Rodriguez,J. and Lazebnik,Y. (1999). Caspase-9 and APAF-1 form an active holoenzyme. Genes Dev. 13, 3179-3184.

Ross,J. and Sullivan,T.D. (1985). Half-lives of beta and gamma globin messenger RNAs and of protein synthetic capacity in cultured human reticulocytes. Blood 66, 1149-1154.

Rothe,M., Pan,M.G., Henzel,W.J., Ayres,T.M., and Goeddel,D.V. (1995). The TNFR2-TRAF signaling complex contains two novel proteins related to baculoviral inhibitor of apoptosis proteins. Cell 83, 1243-1252.

Rothe,M., Wong,S.C., Henzel,W.J., and Goeddel,D.V. (1994). A novel family of putative signal transducers associated with the cytoplasmic domain of the $75 \mathrm{kDa}$ tumor necrosis factor receptor. Cell 78, 681-692.

Rutherford,T., Clegg,J.B., Higgs,D.R., Jones,R.W., Thompson,J., and Weatherall,D.J. (1981). Embryonic erythroid differentiation in the human leukemic cell line K562. Proc. Natl. Acad. Sci. U. S. A 78, 348-352.

Sassa,S. (1976). Sequential induction of heme pathway enzymes during erythroid differentiation of mouse Friend leukemia virus-infected cells. J. Exp. Med. 143, 305-315.

Sattler,M., Liang,H., Nettesheim,D., Meadows,R.P., Harlan,J.E., Eberstadt,M., Yoon,H.S., Shuker,S.B., Chang,B.S., Minn,A.J., Thompson,C.B., and Fesik,S.W. (1997). Structure of Bcl-xL-Bak peptide complex: recognition between regulators of apoptosis. Science 275, 983986.

Savill,J., Fadok,V., Henson,P., and Haslett,C. (1993). Phagocyte recognition of cells undergoing apoptosis. Immunol. Today 14, 131-136.

Scaffidi,C., Fulda,S., Srinivasan,A., Friesen,C., Li,F., Tomaselli,K.J., Debatin,K.M., Krammer,P.H., and Peter,M.E. (1998). Two CD95 (APO-1/Fas) signaling pathways. EMBO J. 17, 1675-1687.

Scaffidi,C., Schmitz,I., Zha,J., Korsmeyer,S.J., Krammer,P.H., and Peter,M.E. (1999). Differential modulation of apoptosis sensitivity in CD95 type I and type II cells. J. Biol. Chem. 274, 22532-22538.

Schall,T.J., Lewis,M., Koller,K.J., Lee,A., Rice,G.C., Wong,G.H., Gatanaga,T., Granger,G.A., Lentz,R., Raab,H., and . (1990). Molecular cloning and expression of a receptor for human tumor necrosis factor. Cell 61, 361-370.

Schickling,O., Stegh,A.H., Byrd,J., and Peter,M.E. (2001). Nuclear localization of DEDD leads to caspase-6 activation through its death effector domain and inhibition of RNA polymerase I dependent transcription. Cell Death. Differ. 8, 1157-1168. 
Schlesinger,P.H., Gross,A., Yin,X.M., Yamamoto,K., Saito,M., Waksman,G., and Korsmeyer,S.J. (1997). Comparison of the ion channel characteristics of proapoptotic BAX and antiapoptotic BCL-2. Proc. Natl. Acad. Sci. U. S. A 94, 11357-11362.

Schmidt,M., Gerlach,F., Avivi,A., Laufs,T., Wystub,S., Simpson,J.C., Nevo,E., SaalerReinhardt,S., Reuss,S., Hankeln,T., and Burmester,T. (2004). Cytoglobin is a respiratory protein in connective tissue and neurons, which is up-regulated by hypoxia. J. Biol. Chem. 279, 8063-8069.

SCHOLANDER,P.F. (1960). Oxygen transport through hemoglobin solutions. Science 131:585-90., 585-590.

Schuller,D.J., Wilks,A., Ortiz de Montellano,P.R., and Poulos,T.L. (1999). Crystal structure of human heme oxygenase-1. Nat. Struct. Biol. 6, 860-867.

Seglen,P.O. and Bohley,P. (1992). Autophagy and other vacuolar protein degradation mechanisms. Experientia 48, 158-172.

Sharpe,J.A., Chan-Thomas,P.S., Lida,J., Ayyub,H., Wood,W.G., and Higgs,D.R. (1992). Analysis of the human alpha globin upstream regulatory element (HS-40) in transgenic mice. EMBO J. 11, 4565-4572.

Shelton,J.G., Steelman,L.S., Lee,J.T., Knapp,S.L., Blalock,W.L., Moye,P.W., Franklin,R.A., Pohnert,S.C., Mirza,A.M., McMahon,M., and McCubrey,J.A. (2003). Effects of the RAF/MEK/ERK and PI3K/AKT signal transduction pathways on the abrogation of cytokinedependence and prevention of apoptosis in hematopoietic cells. Oncogene 22, 2478-2492.

Shibahara,S., Muller,R.M., and Taguchi,H. (1987). Transcriptional control of rat heme oxygenase by heat shock. J. Biol. Chem. 262, 12889-12892.

Shimizu,S., Eguchi,Y., Kamiike,W., Waguri,S., Uchiyama,Y., Matsuda,H., and Tsujimoto,Y. (1996). Retardation of chemical hypoxia-induced necrotic cell death by Bcl-2 and ICE inhibitors: possible involvement of common mediators in apoptotic and necrotic signal transductions. Oncogene 12, 2045-2050.

Shimizu,S., Narita,M., and Tsujimoto,Y. (1999). Bcl-2 family proteins regulate the release of apoptogenic cytochrome c by the mitochondrial channel VDAC. Nature 399, 483-487.

Siddik,Z.H. (2003). Cisplatin: mode of cytotoxic action and molecular basis of resistance. Oncogene 22, 7265-7279.

Silva,M., Benito,A., Sanz,C., Prosper,F., Ekhterae,D., Nunez,G., and Fernandez-Luna,J.L. (1999). Erythropoietin can induce the expression of bcl-x(L) through Stat5 in erythropoietindependent progenitor cell lines. J. Biol. Chem. 274, 22165-22169. 
Simonian,P.L., Grillot,D.A., and Nunez,G. (1997). Bak can accelerate chemotherapy-induced cell death independently of its heterodimerization with Bcl-XL and Bcl-2. Oncogene 15, 1871-1875.

Skurk,C., Maatz,H., Kim,H.S., Yang,J., Abid,M.R., Aird,W.C., and Walsh,K. (2004). The Akt-regulated forkhead transcription factor FOXO3a controls endothelial cell viability through modulation of the caspase-8 inhibitor FLIP. J. Biol. Chem. 279, 1513-1525.

Slee,E.A., Harte,M.T., Kluck,R.M., Wolf,B.B., Casiano,C.A., Newmeyer,D.D., Wang,H.G., Reed,J.C., Nicholson,D.W., Alnemri,E.S., Green,D.R., and Martin,S.J. (1999). Ordering the cytochrome c-initiated caspase cascade: hierarchical activation of caspases-2, -3, -6, -7, -8, and -10 in a caspase-9-dependent manner. J. Cell Biol. 144, 281-292.

Smith,Z.E. and Higgs,D.R. (1999). The pattern of replication at a human telomeric region (16p13.3): its relationship to chromosome structure and gene expression. Hum. Mol. Genet. 8, $1373-1386$.

Solary,E., Bertrand,R., Kohn,K.W., and Pommier,Y. (1993). Differential induction of apoptosis in undifferentiated and differentiated HL-60 cells by DNA topoisomerase I and II inhibitors. Blood 81, 1359-1368.

Song,H.Y., Dunbar,J.D., and Donner,D.B. (1994). Aggregation of the intracellular domain of the type 1 tumor necrosis factor receptor defined by the two-hybrid system. J. Biol. Chem. 269, 22492-22495.

Spadaccini,A., Tilbrook,P.A., Sarna,M.K., Crossley,M., Bieker,J.J., and Klinken,S.P. (1998). Transcription factor erythroid Kruppel-like factor (EKLF) is essential for the erythropoietininduced hemoglobin production but not for proliferation, viability, or morphological maturation. J. Biol. Chem. 273, 23793-23798.

Squier,M.K., Miller,A.C., Malkinson,A.M., and Cohen,J.J. (1994). Calpain activation in apoptosis. J. Cell Physiol 159, 229-237.

Stambolic,V., Suzuki,A., de la Pompa,J.L., Brothers,G.M., Mirtsos,C., Sasaki,T., Ruland,J., Penninger,J.M., Siderovski,D.P., and Mak,T.W. (1998). Negative regulation of PKB/Aktdependent cell survival by the tumor suppressor PTEN. Cell 95, 29-39.

Stegh,A.H., Schickling,O., Ehret,A., Scaffidi,C., Peterhansel,C., Hofmann,T.G., Grummt,I., Krammer,P.H., and Peter,M.E. (1998). DEDD, a novel death effector domain-containing protein, targeted to the nucleolus. EMBO J. 17, 5974-5986.

Susin,S.A., Lorenzo,H.K., Zamzami,N., Marzo,I., Brenner,C., Larochette,N., Prevost,M.C., Alzari,P.M., and Kroemer,G. (1999a). Mitochondrial release of caspase-2 and -9 during the apoptotic process. J. Exp. Med. 189, 381-394. 
Susin,S.A., Lorenzo,H.K., Zamzami,N., Marzo,I., Snow,B.E., Brothers,G.M., Mangion,J., Jacotot,E., Costantini,P., Loeffler,M., Larochette,N., Goodlett,D.R., Aebersold,R., Siderovski,D.P., Penninger,J.M., and Kroemer,G. (1999b). Molecular characterization of mitochondrial apoptosis-inducing factor. Nature 397, 441-446.

Swift, Lorens, Achacoso, and Nolan. Retroviral transduction. Unit 10, 28 (Suppl). 1999. Current Protocols in Immunology.

Taketani,S., Immenschuh,S., Go,S., Sinclair,P.R., Stockert,R.J., Liem,H.H., and Muller,E.U. (1998). Hemopexin from four species inhibits the association of heme with cultured hepatoma cells or primary rat hepatocytes exhibiting a small number of species specific hemopexin receptors. Hepatology 27, 808-814.

Tanaka,M., Suda,T., Haze,K., Nakamura,N., Sato,K., Kimura,F., Motoyoshi,K., Mizuki,M., Tagawa,S., Ohga,S., Hatake,K., Drummond,A.H., and Nagata,S. (1996). Fas ligand in human serum. Nat. Med. 2, 317-322.

Tartaglia,L.A., Pennica,D., and Goeddel,D.V. (1993). Ligand passing: the 75-kDa tumor necrosis factor (TNF) receptor recruits TNF for signaling by the $55-\mathrm{kDa}$ TNF receptor. J. Biol. Chem. 268, 18542-18548.

Thome,M., Schneider,P., Hofmann,K., Fickenscher,H., Meinl,E., Neipel,F., Mattmann,C., Burns,K., Bodmer,J.L., Schroter,M., Scaffidi,C., Krammer,P.H., Peter,M.E., and Tschopp,J. (1997). Viral FLICE-inhibitory proteins (FLIPs) prevent apoptosis induced by death receptors. Nature 386, 517-521.

Thompson,C.B. (1995). Apoptosis in the pathogenesis and treatment of disease. Science 267, 1456-1462.

Ting,C.N., Olson,M.C., Barton,K.P., and Leiden,J.M. (1996). Transcription factor GATA-3 is required for development of the T-cell lineage. Nature 384, 474-478.

Toledano,M.B. and Leonard,W.J. (1991). Modulation of transcription factor NF-kappa B binding activity by oxidation-reduction in vitro. Proc. Natl. Acad. Sci. U. S. A 88, 4328-4332.

Towatari,M., May,G.E., Marais,R., Perkins,G.R., Marshall,C.J., Cowley,S., and Enver,T. (1995). Regulation of GATA-2 phosphorylation by mitogen-activated protein kinase and interleukin-3. J. Biol. Chem. 270, 4101-4107.

Troy,C.M. and Shelanski,M.L. (2003). Caspase-2 redux. Cell Death. Differ. 10, 101-107.

Truneh,A., $\quad$ Sharma,S., $\quad$ Silverman,C., $\quad$ Khandekar,S., $\quad$ Reddy,M.P., $\quad$ Deen,K.C., McLaughlin,M.M., Srinivasula,S.M., Livi,G.P., Marshall,L.A., Alnemri,E.S., Williams,W.V., and Doyle,M.L. (2000). Temperature-sensitive differential affinity of TRAIL for its receptors. DR5 is the highest affinity receptor. J. Biol. Chem. 275, 23319-23325. 
Tsai,S.F., Martin,D.I., Zon,L.I., D'Andrea,A.D., Wong,G.G., and Orkin,S.H. (1989). Cloning of cDNA for the major DNA-binding protein of the erythroid lineage through expression in mammalian cells. Nature 339, 446-451.

Tsang,A.P., Visvader,J.E., Turner,C.A., Fujiwara,Y., Yu,C., Weiss,M.J., Crossley,M., and Orkin,S.H. (1997). FOG, a multitype zinc finger protein, acts as a cofactor for transcription factor GATA-1 in erythroid and megakaryocytic differentiation. Cell 90, 109-119.

Tsushima,H., Urata,Y., Miyazaki,Y., Fuchigami,K., Kuriyama,K., Kondo,T., and Tomonaga,M. (1997). Human erythropoietin receptor increases GATA-2 and Bcl-xL by a protein kinase C-dependent pathway in human erythropoietin-dependent cell line AS-E2. Cell Growth Differ. 8, 1317-1328.

Tufarelli,C., Hardison,R., Miller,W., Hughes,J., Clark,K., Ventress,N., Frischauf,A.M., and Higgs,D.R. (2004). Comparative analysis of the alpha-like globin clusters in mouse, rat, and human chromosomes indicates a mechanism underlying breaks in conserved synteny. Genome Res. 14, 623-630.

Van de,C.M., Van Loo,G., Pype,S., Van Criekinge,W., Van,d.B., I, Molemans,F., Fiers,W., Declercq,W., and Vandenabeele,P. (1998). Identification of a new caspase homologue: caspase-14. Cell Death. Differ. 5, 838-846.

Van de,C.M., Vandenabeele,P., Declercq,W., Van,d.B., I, Van Loo,G., Molemans,F., Schotte,P., Van Criekinge,W., Beyaert,R., and Fiers,W. (1997). Characterization of seven murine caspase family members. FEBS Lett. 403, 61-69.

Vaux,D.L., Cory,S., and Adams,J.M. (1988). Bcl-2 gene promotes haemopoietic cell survival and cooperates with c-myc to immortalize pre-B cells. Nature 335, 440-442.

Vaux,D.L., Haecker,G., and Strasser,A. (1994). An evolutionary perspective on apoptosis. Cell 76, 777-779.

Verhagen,A.M., Ekert,P.G., Pakusch,M., Silke,J., Connolly,L.M., Reid,G.E., Moritz,R.L., Simpson,R.J., and Vaux,D.L. (2000). Identification of DIABLO, a mammalian protein that promotes apoptosis by binding to and antagonizing IAP proteins. Cell 102, 43-53.

Visvader,J.E., Crossley,M., Hill,J., Orkin,S.H., and Adams,J.M. (1995). The C-terminal zinc finger of GATA-1 or GATA-2 is sufficient to induce megakaryocytic differentiation of an early myeloid cell line. Mol. Cell Biol. 15, 634-641.

Volloch,V. and Housman,D. (1981). Stability of globin mRNA in terminally differentiating murine erythroleukemia cells. Cell 23, 509-514.

von Gise,A., Lorenz,P., Wellbrock,C., Hemmings,B., Berberich-Siebelt,F., Rapp,U.R., and Troppmair,J. (2001). Apoptosis suppression by Raf-1 and MEK1 requires MEK- and phosphatidylinositol 3-kinase-dependent signals. Mol. Cell Biol. 21, 2324-2336. 
Walczak,H. and Krammer,P.H. (2000). The CD95 (APO-1/Fas) and the TRAIL (APO-2L) apoptosis systems. Exp. Cell Res. 256, 58-66.

Walker,N.P., Talanian,R.V., Brady,K.D., Dang,L.C., Bump,N.J., Ferenz,C.R., Franklin,S., Ghayur,T., Hackett,M.C., Hammill,L.D., and . (1994). Crystal structure of the cysteine protease interleukin-1 beta-converting enzyme: a (p20/p10)2 homodimer. Cell 78, 343-352.

Wang,X., Kiledjian,M., Weiss,I.M., and Liebhaber,S.A. (1995). Detection and characterization of a 3' untranslated region ribonucleoprotein complex associated with human alpha-globin mRNA stability. Mol. Cell Biol. 15, 1769-1777.

Wang,Z. and Kiledjian,M. (2000). The poly(A)-binding protein and an mRNA stability protein jointly regulate an endoribonuclease activity. Mol. Cell Biol. 20, 6334-6341.

Wei,M.C., Lindsten,T., Mootha,V.K., Weiler,S., Gross,A., Ashiya,M., Thompson,C.B., and Korsmeyer,S.J. (2000). tBID, a membrane-targeted death ligand, oligomerizes BAK to release cytochrome c. Genes Dev. 14, 2060-2071.

Weiss,I.M. and Liebhaber,S.A. (1995). Erythroid cell-specific mRNA stability elements in the alpha 2-globin 3' nontranslated region. Mol. Cell Biol. 15, 2457-2465.

Weiss,M.J. and Orkin,S.H. (1995a). GATA transcription factors: key regulators of hematopoiesis. Exp. Hematol. 23, 99-107.

Weiss,M.J. and Orkin,S.H. (1995b). Transcription factor GATA-1 permits survival and maturation of erythroid precursors by preventing apoptosis. Proc. Natl. Acad. Sci. U. S. A 92, 9623-9627.

Weiss,M.J., Yu,C., and Orkin,S.H. (1997). Erythroid-cell-specific properties of transcription factor GATA-1 revealed by phenotypic rescue of a gene-targeted cell line. Mol. Cell Biol. 17, 1642-1651.

Wick,M.J., Dong,L.Q., Riojas,R.A., Ramos,F.J., and Liu,F. (2000). Mechanism of phosphorylation of protein kinase $\mathrm{B} / \mathrm{Akt}$ by a constitutively active 3-phosphoinositidedependent protein kinase-1. J. Biol. Chem. 275, 40400-40406.

Wiley,S.R., Schooley,K., Smolak,P.J., Din,W.S., Huang,C.P., Nicholl,J.K., Sutherland,G.R., Smith,T.D., Rauch,C., Smith,C.A., and . (1995). Identification and characterization of a new member of the TNF family that induces apoptosis. Immunity. 3, 673-682.

Wilson,B.E., Mochon,E., and Boxer,L.M. (1996). Induction of bcl-2 expression by phosphorylated CREB proteins during B-cell activation and rescue from apoptosis. Mol. Cell Biol. 16, 5546-5556.

Wittenberg,B.A., Wittenberg,J.B., and Caldwell,P.R. (1975). Role of myoglobin in the oxygen supply to red skeletal muscle. J. Biol. Chem. 250, 9038-9043. 
Worthington,R.E., Bossie-Codreanu,J., and Van Zant,G. (1987). Quantitation of erythroid differentiation in vitro using a sensitive colorimetric assay for hemoglobin. Exp. Hematol. 15, $85-92$.

Wride,M.A., Mansergh,F.C., Adams,S., Everitt,R., Minnema,S.E., Rancourt,D.E., and Evans,M.J. (2003). Expression profiling and gene discovery in the mouse lens. Mol. Vis. 9:360-96., 360-396.

Wyllie,A.H. (1980). Glucocorticoid-induced thymocyte apoptosis is associated with endogenous endonuclease activation. Nature 284, 555-556.

Yamaguchi,H. and Wang,H.G. (2001). The protein kinase PKB/Akt regulates cell survival and apoptosis by inhibiting Bax conformational change. Oncogene 20, 7779-7786.

Yamamoto,M., Ko,L.J., Leonard,M.W., Beug,H., Orkin,S.H., and Engel,J.D. (1990). Activity and tissue-specific expression of the transcription factor NF-E1 multigene family. Genes Dev. 4, 1650-1662.

Yang,E., Zha,J., Jockel,J., Boise,L.H., Thompson,C.B., and Korsmeyer,S.J. (1995). Bad, a heterodimeric partner for Bcl-XL and Bcl-2, displaces Bax and promotes cell death. Cell 80, 285-291.

Yang,J.Y. and Widmann,C. (2001). Antiapoptotic signaling generated by caspase-induced cleavage of RasGAP. Mol. Cell Biol. 21 , 5346-5358.

Yarmolinsky,M.B. (1995). Programmed cell death in bacterial populations. Science 267, 836837.

Yoshida,E., Ohmura,S., Sugiki,M., Anai,K., and Maruyama,M. (2001). A novel function of extraerythrocytic hemoglobin: identification of globin as a stimulant of plasminogen activator biosynthesis in human fibroblasts. Thromb. Haemost. 86, 1521-1527.

Yu,Y.L., Chiang,Y.J., and Yen,J.J. (2002). GATA factors are essential for transcription of the survival gene E4bp4 and the viability response of interleukin-3 in $\mathrm{Ba} / \mathrm{F} 3$ hematopoietic cells. J. Biol. Chem. 277, 27144-27153.

Yuan,J., Bunyaratvej,A., Fucharoen,S., Fung,C., Shinar,E., and Schrier,S.L. (1995). The instability of the membrane skeleton in thalassemic red blood cells. Blood 86, 3945-3950.

Zamzami,N., Marchetti,P., Castedo,M., Zanin,C., Vayssiere,J.L., Petit,P.X., and Kroemer,G. (1995). Reduction in mitochondrial potential constitutes an early irreversible step of programmed lymphocyte death in vivo. J. Exp. Med. 181, 1661-1672.

Zha,H. and Reed,J.C. (1997). Heterodimerization-independent functions of cell death regulatory proteins Bax and Bcl-2 in yeast and mammalian cells. J. Biol. Chem. 272, 3148231488. 
Zha,J., Harada,H., Yang,E., Jockel,J., and Korsmeyer,S.J. (1996). Serine phosphorylation of death agonist BAD in response to survival factor results in binding to 14-3-3 not BCL-X(L). Cell 87, 619-628.

Zhang,H., Heim,J., and Meyhack,B. (1998). Redistribution of Bax from cytosol to membranes is induced by apoptotic stimuli and is an early step in the apoptotic pathway. Biochem. Biophys. Res. Commun. 251, 454-459.

Zimmermann,S. and Moelling,K. (1999). Phosphorylation and regulation of Raf by Akt (protein kinase B). Science 286, 1741-1744.

Zorbas,H., Rein,T., Krause,A., Hoffmann,K., and Winnacker,E.L. (1992). Nuclear factor I (NF I) binds to an NF I-type site but not to the CCAAT site in the human alpha-globin gene promoter. J. Biol. Chem. 267, 8478-8484.

Zornig,M., Hueber,A., Baum,W., and Evan,G. (2001). Apoptosis regulators and their role in tumorigenesis. Biochim. Biophys. Acta 1551, F1-37.

Zou,H., Li,Y., Liu,X., and Wang,X. (1999). An APAF-1.cytochrome c multimeric complex is a functional apoptosome that activates procaspase-9. J. Biol. Chem. 274, 11549-11556. 